\title{
Enantioselective Construction of Chiral Sulfides via Catalytic Electrophilic Azidothiolation and Oxythiolation of $N$-Allyl Sulfonamides
}

\author{
Yaoyu Liang and Xiaodan Zhao* \\ Institute of Organic Chemistry \& MOE Key Laboratory of Bioinorganic and Synthetic Chemistry \\ School of Chemistry, Sun Yat-Sen University, Guangzhou 510275, China \\ E-mail:zhaoxd3@mail.sysu.edu.cn
}

Table of Contents

1. General considerations

2. Condition evaluation

3. Synthetic procedures and characterization data of alkene substrates

4. Synthetic procedures and characterization data of sulfur reagents

5. Synthetic procedures and characterization data of catalysts

6. Catalytic enantioselective difunctionalization of $N$-allyl sulfonamides and characterization data of difunctionalization products

7. Transformations of products

8. Mechanistic studies

9. X-ray crystallographic data of $\mathbf{8}$ 


\section{General considerations}

Unless otherwise noted, commercial reagents were purchased from Alfa Aesar, TCI, $J \& K$, Energy, Macklin or Adamas and used without further purification. Reaction solvents THF, DCM and MeCN were purified by PureSolv MD-5 (Innovative Technology). DCE and $\mathrm{CHCl}_{3}$ were dried over $\mathrm{CaH}_{2}$ prior to use. All the reactions were carried out with oven-dried glassware. All catalytic reactions were conducted without special care. Analytical thin layer chromatography was performed on 0.20 mm silica gel HSGF-254 plates (Huanghai, China), and visualized under $254 \mathrm{~nm} \mathrm{UV}$ light or by staining with potassium permanganate. Column chromatography was performed on 200-300 mesh silica gel (Huanghai, China).

${ }^{1} \mathrm{H},{ }^{19} \mathrm{~F},{ }^{31} \mathrm{P}$ and ${ }^{13} \mathrm{C}$ NMR spectra were recorded on an AVANCE III $400 \mathrm{MHz}$ spectrometer at ambient temperature. ${ }^{1} \mathrm{H}$ NMR spectra are referred to the TMS signal $(\delta=0 \mathrm{ppm})$ and ${ }^{13} \mathrm{C}$ NMR spectra are referred to the residual solvent signal $(\delta=77.16$ ppm). Data for ${ }^{1} \mathrm{H}$ NMR are reported as follows: chemical shift $(\delta \mathrm{ppm})$, multiplicity ( $\mathrm{s}=$ singlet, $\mathrm{d}=$ doublet, $\mathrm{t}=$ triplet, $\mathrm{q}=$ quartet, $\mathrm{m}=$ multiplet $)$, coupling constant $(\mathrm{Hz})$, integration. Data for ${ }^{13} \mathrm{C}$ NMR, ${ }^{19} \mathrm{~F}$ NMR and ${ }^{31} \mathrm{P}$ NMR are reported as follows: chemical shift $(\delta \mathrm{ppm})$, multiplicity $(\mathrm{q}=$ quartet$)$, coupling constant $(\mathrm{Hz})$.

High resolution mass spectra of new compounds were recorded on LTQ Orbitrap Elite LC/MS (ESI) at analytical center of Sun Yat-Sen University. Infrared (IR) spectra were recorded on Bruker FT-IR spectrometer (EQUINOX 55) at analytical center of Sun Yat-Sen University and reported in wave numbers $\left(\mathrm{cm}^{-1}\right)$. Melting points were determined on a YUHUA X-5 micro melting point apparatus. Enantiomeric excesses were determined by HPLC analysis on Shimadzu HPLC units including the following instruments: LC-20AT pump, SPD-M20A detector, Sil-20A autosampler, CTO-20A column oven, Waters 1525 binary HPLC pumb, 2998 potodiode array detector, 2707 autosampler and Dacicel Chiralpak IA, IC, ID, OJ-H, OD-H, AD-H columns. Optical rotations were recorded on an Anton Paar MCD-200 polarimeter.

All the racemic products were obtained by using $\mathrm{PhSePh}$ as the catalyst at $-78{ }^{\circ} \mathrm{C}$ to 0 ${ }^{\circ} \mathrm{C}$ without a note. The diastereoselectivities of products were determined by crude ${ }^{1} \mathrm{H}$ NMR or HPLC. 


\section{Condition evaluation}

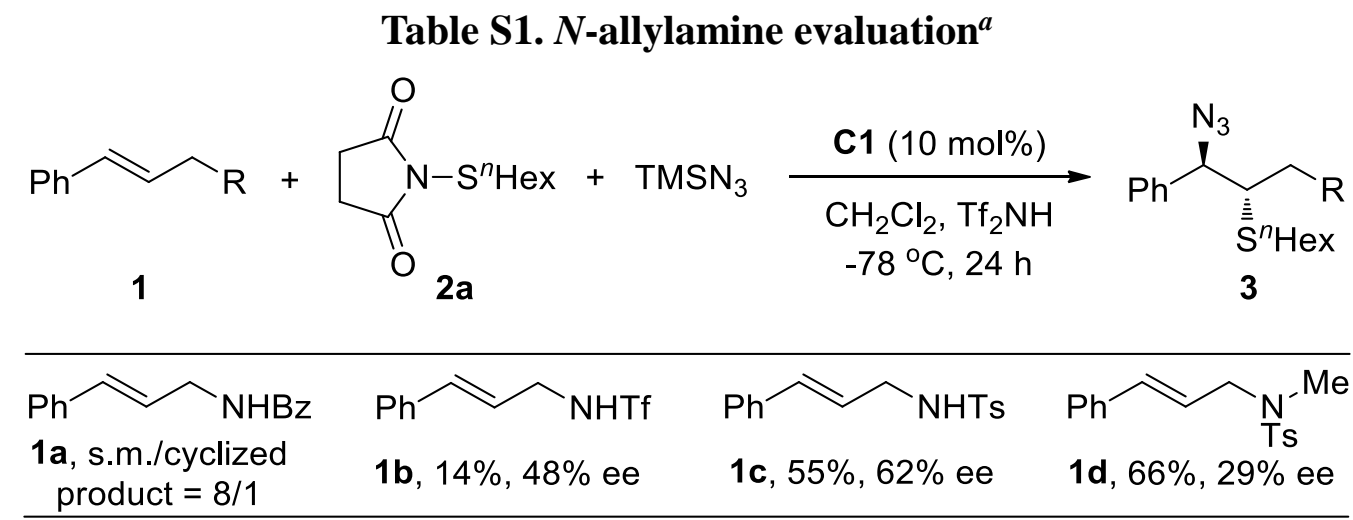

${ }^{a}$ Conditions: 1 ( 0.05 mmol), $\mathbf{2 a}$ (1.5 equiv), $\mathrm{TMSN}_{3}$ (2.0 equiv), $\mathrm{Tf}_{2} \mathrm{NH}$ (2.0 equiv), $\mathrm{C1}(10 \mathrm{~mol} \%), \mathrm{CH}_{2} \mathrm{Cl}_{2}(2.0 \mathrm{~mL}),-78{ }^{\circ} \mathrm{C}, 24 \mathrm{~h}$. Yields refer to NMR yield using quinoline as the internal standard and ee values were determined by HPLC analysis.

\section{Table S2. Solvent evaluation ${ }^{a}$}

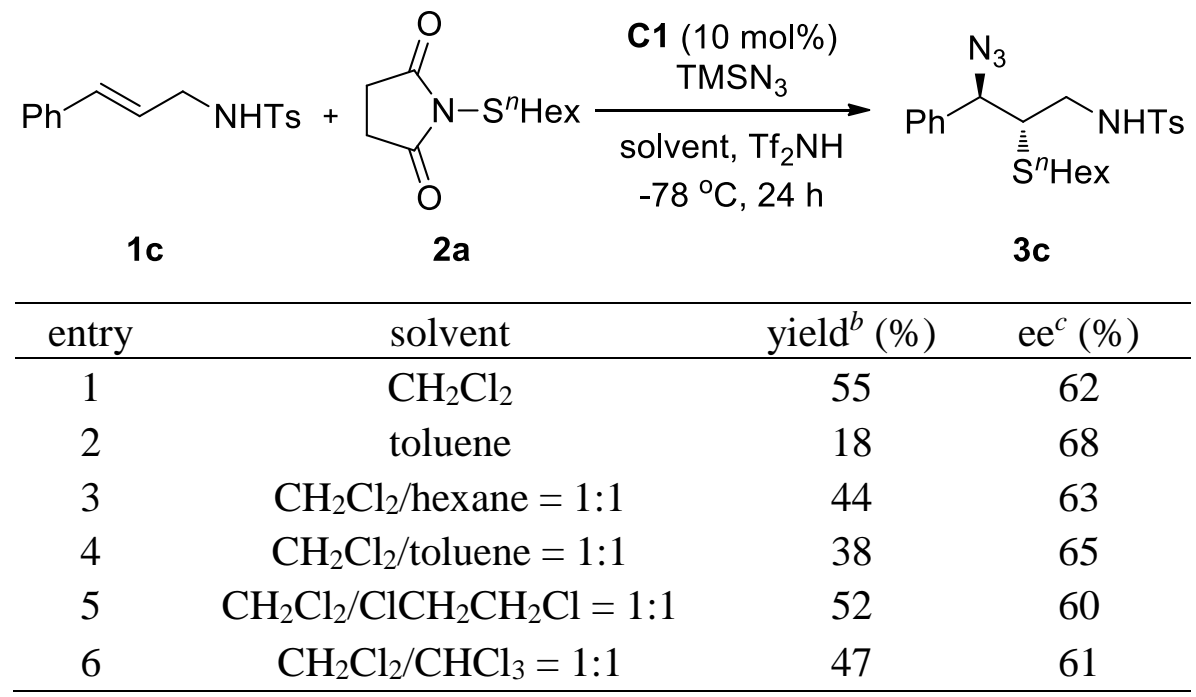

${ }^{a}$ Conditions: 1c $(0.05 \mathrm{mmol}), \mathbf{2 a}$ (1.5 equiv), $\mathrm{TMSN}_{3}$ (2.0 equiv), $\mathrm{Tf}_{2} \mathrm{NH}$ (2.0 equiv), C1 (10 mol\%), solvent $(2.0 \mathrm{~mL}),-78{ }^{\circ} \mathrm{C}, 24 \mathrm{~h} .{ }^{b}$ Refers to NMR yield using quinoline as the internal standard. ${ }^{c}$ Determined by HPLC analysis. All the diastereoselectivities are $>99: 1$. 
Table S3. Catalyst evaluation ${ }^{a}$
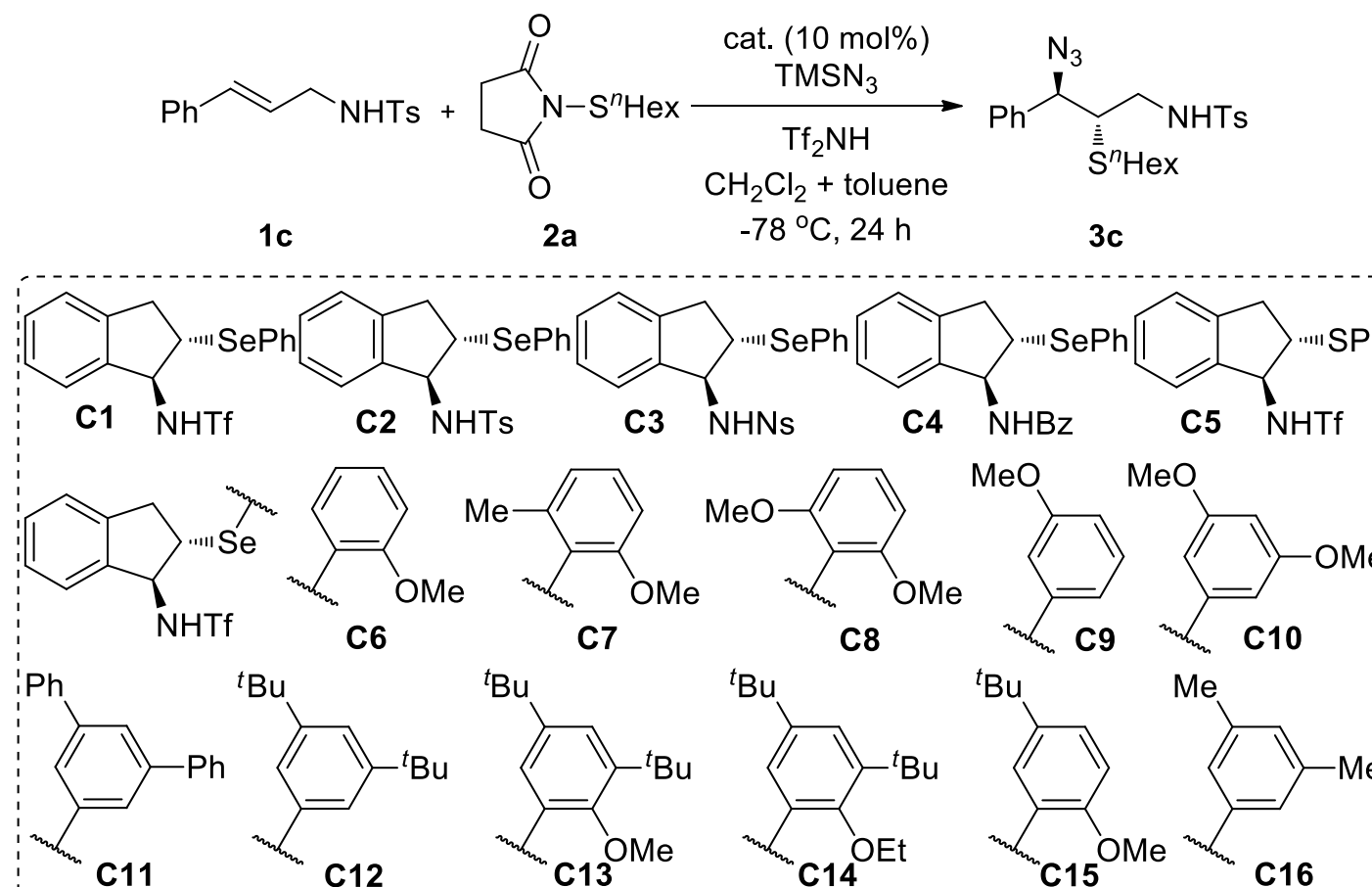
$\mathrm{MeO} \quad \mathrm{MeO}$
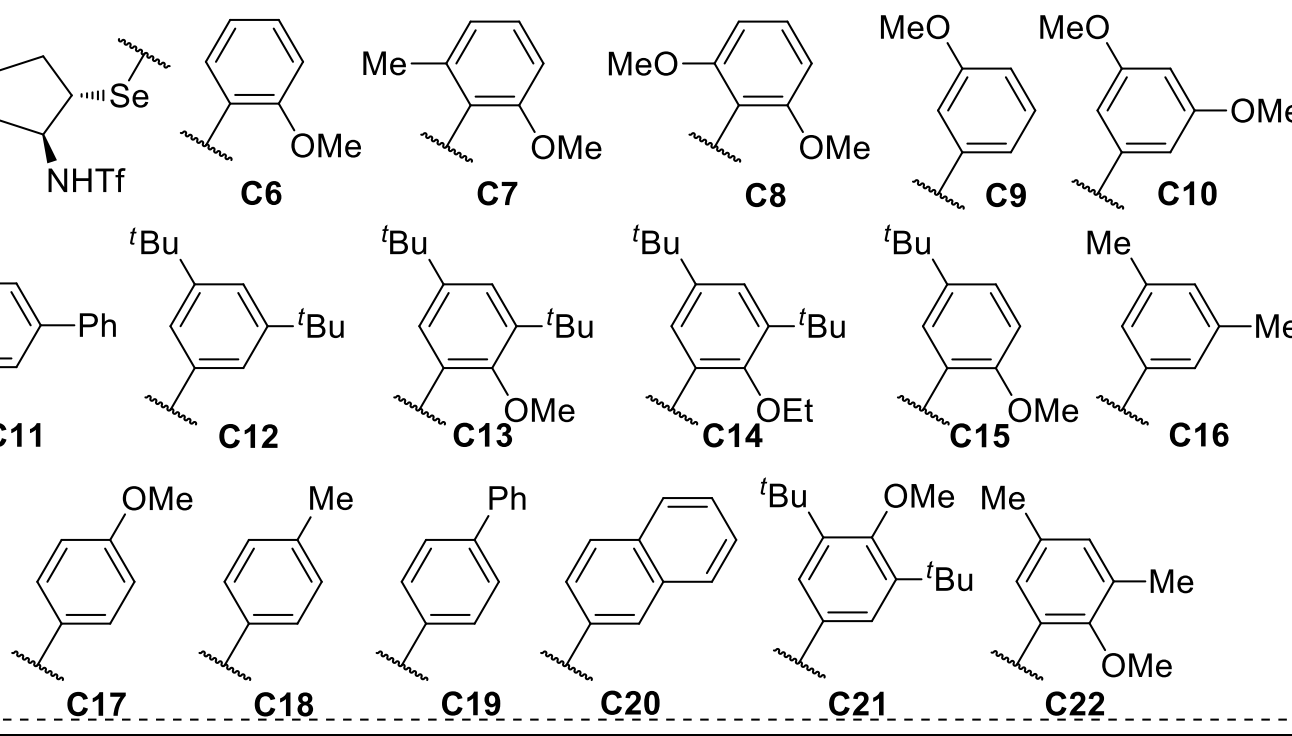

\begin{tabular}{cccc}
\hline entry & cat. & yield $^{b}(\%)$ & $\mathrm{ee}^{c}(\%)$ \\
\hline 1 & C1 & 38 & 65 \\
2 & C2 & 22 & 32 \\
3 & C3 & 42 & 48 \\
4 & C4 & 46 & $<3$ \\
5 & C5 & 32 & 59 \\
6 & C6 & 64 & 69 \\
7 & C7 & 88 & 36 \\
8 & C8 & 92 & 34 \\
9 & C9 & 59 & 66 \\
10 & C10 & 82 & 68 \\
11 & C11 & 62 & 76 \\
12 & C12 & 73 & 77 \\
13 & C13 & 96 & 92 \\
$14^{d}$ & C13 & 37 & 88 \\
15 & C14 & 95 & 94 \\
16 & C15 & 80 & 59 \\
17 & C16 & 24 & 57 \\
18 & C17 & 76 & 67 \\
19 & C18 & 58 & 65 \\
\hline
\end{tabular}




\begin{tabular}{llll}
\hline 20 & C19 & 65 & 65 \\
21 & C20 & 32 & 42 \\
22 & C21 & 69 & 58 \\
23 & C22 & 87 & 70 \\
\hline
\end{tabular}

${ }^{a}$ Conditions: 1c $(0.05 \mathrm{mmol}), \mathbf{2 a}$ (1.5 equiv), $\mathrm{TMSN}_{3}$ (2.0 equiv), $\mathrm{Tf}_{2} \mathrm{NH}$ (2.0 equiv), cat. $(10 \mathrm{~mol} \%), \mathrm{CH}_{2} \mathrm{Cl}_{2}(1.0 \mathrm{~mL})+$ toluene $(1.0 \mathrm{~mL}),-78{ }^{\circ} \mathrm{C}, 24 \mathrm{~h} .{ }^{b}$ Refers to NMR yield using quinoline as the internal standard. ${ }^{c}$ Determined by HPLC analysis. All the diastereoselectivities are $>99: 1 .{ }^{d}$ With the corresponding sulfur catalyst of C13.

Note: $(Z)$-configured $N$-allyl sulfonamide was also tested under the similar conditions. When the reaction was carried out with electrophilic alkylthio $\left(\mathrm{AlkylS}^{+}\right)$reagent, the reaction did not occur. However, electrophilic arylthio $\left(\mathrm{ArS}^{+}\right)$reagent gave the desired product in $90 \%$ NMR yield with only $20 \%$ ee. The results were showed as follows.
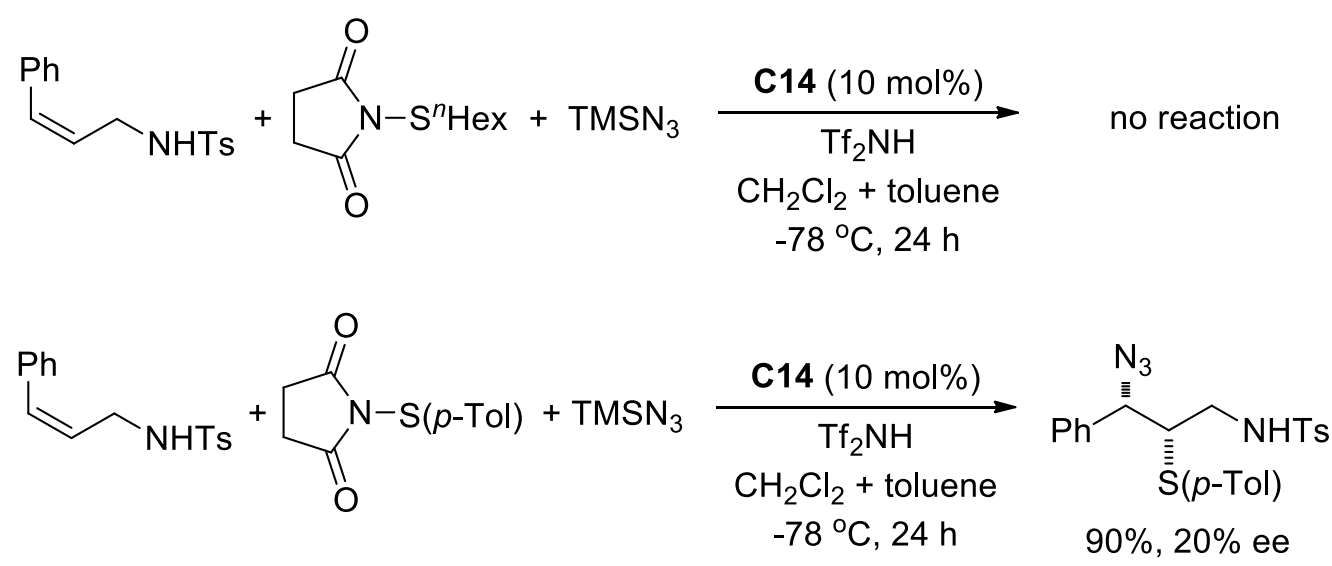

\section{Synthetic procedures and characterization data of alkene}

\section{substrates}

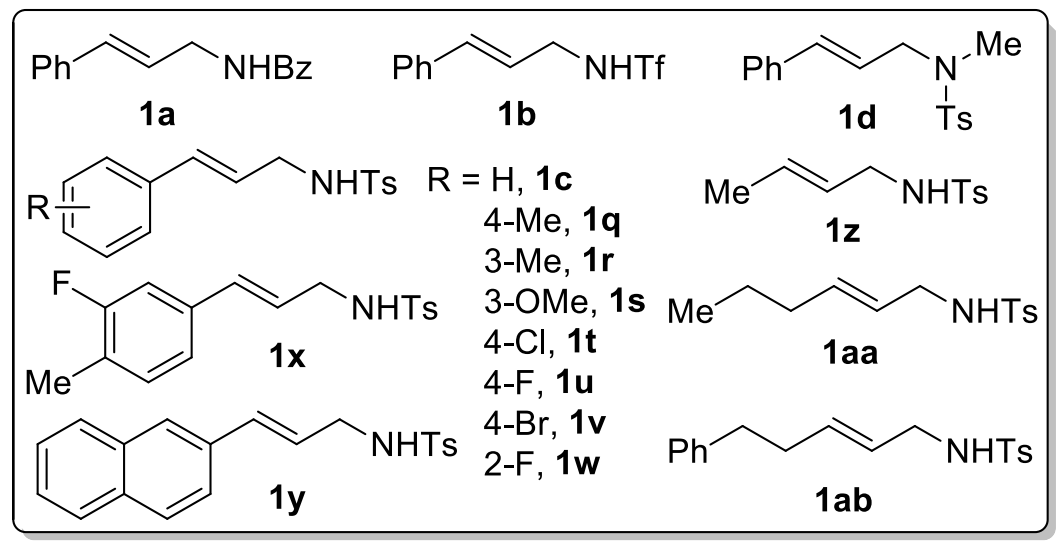




\subsection{Synthesis of 1a}

The procedure for the synthesis of compound $\mathbf{1 a}$ is based on the known procedure. ${ }^{1,2}$ Its spectral data was in accordance with the reported in the literature. ${ }^{2}$

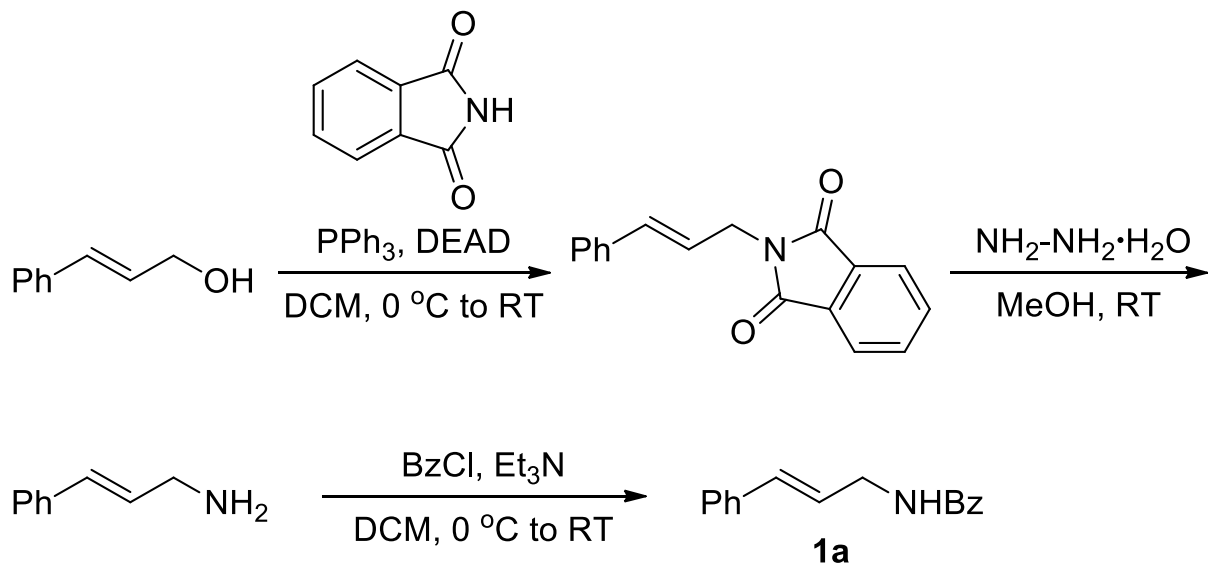

To a solution of phthalimide ( $809 \mathrm{mg}, 5.5 \mathrm{mmol})$ and $\mathrm{PPh}_{3}(1.44 \mathrm{~g}, 5.5 \mathrm{mmol})$ in DCM $(10 \mathrm{~mL})$ was added cinnamyl alcohol $(671 \mathrm{mg}, 5.0 \mathrm{mmol})$ under nitrogen atmosphere. The solution was cooled to $0{ }^{\circ} \mathrm{C}$ and DEAD (diethoxycarbonyldiazene, $866 \mu \mathrm{L}, 5.5 \mathrm{mmol}$ ) was added dropwise. After the addition, the resulting solution was allowed to warm to room temperature and stirred overnight. Water $(10 \mathrm{~mL})$ was added. Then, the mixture was extracted with DCM $(15 \mathrm{~mL} \times 3)$. The combined organic phases were dried over anhydrous $\mathrm{Na}_{2} \mathrm{SO}_{4}$, filtered, and concentrated under reduced pressure. The residue was purified by silica gel flash collumn chromotography (eluent: $\mathrm{PE} / \mathrm{EtOAc}=20 / 1$ to $5 / 1, \mathrm{v} / \mathrm{v}$ ) to give $N$-allylphthalimide product as a white solid $(1.17 \mathrm{~g}, 89 \%$ yield $)$.

A solution of $N$-allylphthalimide $(1.17 \mathrm{~g}, 4.5 \mathrm{mmol})$ and hydrazine hydrate $(437 \mu \mathrm{L}$, $9.0 \mathrm{mmol})$ in methanol $(20 \mathrm{~mL})$ was stirred at room temperature for $12 \mathrm{~h}$. Concentrated $\mathrm{HCl}$ was added until $\mathrm{pH}<1$. The solution was refluxed for $30 \mathrm{~min}$, and then recooled and filtered to remove the phthalhydrazide. The precipitate was washed with cold $\mathrm{MeOH}$. The filtrate was concentrated and the residue was dissolved in EtOH. The solution was refiltered to remove final traces of phthalhydrazide. The filtrates were concentrated. Then, the residue was dissolved in water $(20 \mathrm{~mL})$, basified with $\mathrm{NaOH}$ until $\mathrm{pH}>10$, and extracted with EtOAc $(20 \mathrm{~mL} \times 3)$. The extracts were dried over anhydrous $\mathrm{Na}_{2} \mathrm{SO}_{4}$, filtered, and concentrated under reduced pressure to give (E)-3-phenylprop-2-en-1-amine as a pale yellow oil (431 $\mathrm{mg}, 72 \%$ yield). The crude product was used for the next step without further purification.

(E)-3-Phenylprop-2-en-1-amine (266 mg, $2.0 \mathrm{mmol}$ ) was dissolved in DCM (10 mL). Triethylamine $(417 \mu \mathrm{L}, 3.0 \mathrm{mmol})$ and benzoyl chloride $(255 \mu \mathrm{L}, 2.2 \mathrm{mmol})$ were added dropwise at $0{ }^{\circ} \mathrm{C}$. The solution was stirred for $4 \mathrm{~h}$ at room temperature, and then diluted with water $(10 \mathrm{~mL})$ and extracted with $\mathrm{DCM}(15 \mathrm{~mL} \times 3)$. Organic extracts were dried over $\mathrm{Na}_{2} \mathrm{SO}_{4}$ and filtered. After concentration, the residue was purified by 
silica gel column chromatography (eluent: $\mathrm{PE} / \mathrm{EtOAc}=20 / 1$ to $5 / 1, \mathrm{v} / \mathrm{v}$ ) to give $N$-cinnamylbenzamide 1a as a white solid (346 mg, 73\% yield).

\section{2 Synthesis of $\mathbf{1 b}$}

The procedure for the synthesis of compound $\mathbf{1 b}$ is based on the known procedure and its spectral data was in accordance with the reported in the literature. ${ }^{3}$

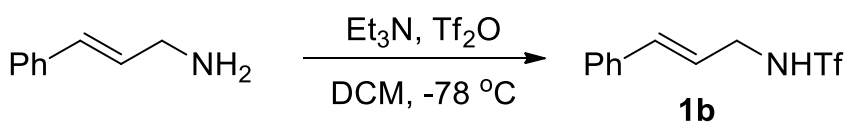

(E)-3-Phenylprop-2-en-1-amine was prepared as described above. To a stirred solution of amine (266 mg, $2.0 \mathrm{mmol})$ in DCM (10 mL) was added triethylamine (417 $\mu \mathrm{L}, 3.0 \mathrm{mmol})$. Trifluoromethanesulfonic anhydride $(370 \mu \mathrm{L}, 2.2 \mathrm{mmol})$ was added dropwise at $-78{ }^{\circ} \mathrm{C}$ and the solution was stirred for $1 \mathrm{~h}$ at that temperature before being quenched by water $(10 \mathrm{~mL})$. The organic layer was separated and the aqueous layer was extracted with DCM $(15 \mathrm{~mL} \times 3)$. The combined organic phases were dried over $\mathrm{Na}_{2} \mathrm{SO}_{4}$ and filtered. After concentration, the residue was purified by silica gel flash chromatography (eluent: PE/EtOAc $=20 / 1$ to $5 / 1, \mathrm{v} / \mathrm{v}$ ) to give the desired product $\mathbf{1 b}$ as a white solid (329 $\mathrm{mg}, 62 \%$ yield).

\section{3 Synthesis of 1c, 1q-1y and 1aa-1ab}

General procedure for the synthesis of compounds 1c, 1q-1y and 1aa-1ab is based on the known procedure. ${ }^{1,4}$ Compounds $1 \mathbf{c},{ }^{1} \mathbf{1 q},{ }^{1} \mathbf{1 r}-1 \mathbf{s},{ }^{5} \mathbf{1 t}-\mathbf{1 u},{ }^{1} \mathbf{1 w},{ }^{5} \mathbf{1 y}$ and $\mathbf{1 a a}{ }^{1}$ were known and their spectral data were in accordance with the reported in the literature.

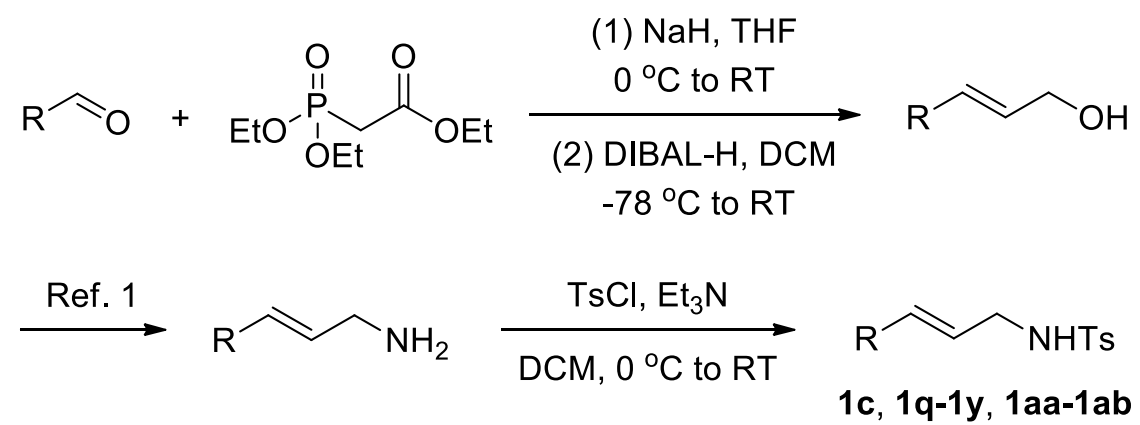

The reaction was conducted by modifying the literature procedure. ${ }^{4}$ To a suspension of $\mathrm{NaH}(60 \%, 220 \mathrm{mg}, 5.5 \mathrm{mmol})$ in $\mathrm{THF}(10 \mathrm{~mL})$ at $0{ }^{\circ} \mathrm{C}$ was added dropwise triethyl phosphonoacetate $(1.09 \mathrm{~mL}, 5.5 \mathrm{mmol})$. The mixture was stirred at $0{ }^{\circ} \mathrm{C}$ for $30 \mathrm{~min}$. Aldehyde (5.0 mmol, 1.0 equiv) was then added dropwise. The mixture was allowed to warm to room temperature and stirred until complete consumption of the aldehyde (about $4 \mathrm{~h}$ ). Then, the reaction was quenched with $5 \mathrm{~mL}$ saturated aqueous $\mathrm{NH}_{4} \mathrm{Cl}$. The aqueous layer was extracted with EtOAc $(10 \mathrm{~mL} \times 3)$. The combined organic layers were washed with brine, dried over $\mathrm{Na}_{2} \mathrm{SO}_{4}$, and concentrated under 
reduced pressure. The crude $\alpha, \beta$-unsaturated ester was used for the next step without further purification.

To a solution of the above obtained $\alpha, \beta$-unsaturated ester (1.0 equiv) in DCM ( $0.2 \mathrm{M})$ at $-78{ }^{\circ} \mathrm{C}$ was added dropwise DIBAL-H (2.1 equiv). The mixture was stirred for $2 \mathrm{~h}$, and then allowed to warm to room temperature. Saturated aqueous $\mathrm{NH}_{4} \mathrm{Cl}$ was added slowly. The resulting mixture was stirred for another $1 \mathrm{~h}$ and then extracted with DCM $(15 \mathrm{~mL} \times 3)$. The combined organic layers were washed with brine, dried over $\mathrm{Na}_{2} \mathrm{SO}_{4}$, and concentrated under reduced pressure. The crude allyl alcohol was used for the next step withtout further purification.

To a solution of allyl amide in DCM $(0.2 \mathrm{M})$ were added triethylamine (1.5 equiv) and tosyl chloride (1.1 equiv) at $0{ }^{\circ} \mathrm{C}$. The resulting solution was stirred for $4 \mathrm{~h}$ at room temperature, and then diluted with water $(5 \mathrm{~mL})$ and extracted with DCM (15 $\mathrm{mL} \times 3)$. Organic extracts were dried over $\mathrm{Na}_{2} \mathrm{SO}_{4}$ and filtered. After concentration, the residue was purified by flash silica gel column chromatography to yield the corresponding product.

\section{4 Synthesis of $1 d$}

The procedure for the synthesis of compound 1d is based on the known procedure and its spectral data was in accordance with the reported in the literature. ${ }^{6}$
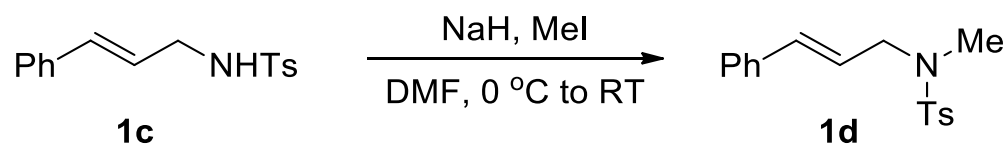

To a solution of $1 \mathbf{c}(287 \mathrm{mg}, 1.0 \mathrm{mmol})$ in DMF $(10 \mathrm{~mL})$ was added $\mathrm{NaH}(60 \%, 48.0$ $\mathrm{mg}, 1.2 \mathrm{mmol}$ ) at $0{ }^{\circ} \mathrm{C}$. The resulting mixture was stirred at room temperature for 30 min, after which it was cooled to $0{ }^{\circ} \mathrm{C}$ and treated with methyl iodide $(170 \mathrm{mg}, 1.2$ mmol). The mixture was warmed to room temperature and stirred overnight. Then, the mixture was poured into water $(10 \mathrm{~mL})$ and extracted with EtOAc $(15 \mathrm{~mL} \times 3)$. The combined organic layers were washed with water $(20 \mathrm{~mL} \times 3)$, dried with $\mathrm{Na}_{2} \mathrm{SO}_{4}$, and concentrated to give the crude mixture, which was purified by flash columm chromatography $(\mathrm{PE} / \mathrm{EtOAc}=15 / 1$ to $5 / 1, \mathrm{v} / \mathrm{v})$ to give the final product $\mathbf{1 d}$ as a white solid (289 $\mathrm{mg}, 96 \%$ yield).

\section{5 Synthesis of $1 z$}

The procedure for the synthesis of compound $\mathbf{1 z}$ is based on the known procedure and its spectral data was in accordance with the reported in the literature. ${ }^{7}$

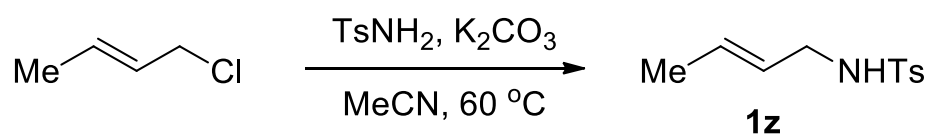


To a solution of $\mathrm{TsNH}_{2}(685 \mathrm{mg}, 4.0 \mathrm{mmol})$ and $\mathrm{K}_{2} \mathrm{CO}_{3}(553 \mathrm{mg}, 4.0 \mathrm{mmol})$ in $\mathrm{MeCN}$ $(10 \mathrm{~mL})$ was added $(E)-1$-chlorobut-2-ene $(196 \mu \mathrm{L}, 2.0 \mathrm{mmol})$. The mixture was heated to $60{ }^{\circ} \mathrm{C}$ and stirred overnight. Then, the mixture was cooled to room temperature and water $(20 \mathrm{~mL})$ was added. The resulting mixture was extracted with EtOAc $(20 \mathrm{~mL} \times 3)$. The combined organic layers were washed with water, dried over anhydrous $\mathrm{Na}_{2} \mathrm{SO}_{4}$, filtered, and concentrated under reduced pressure. The residue was purified by flash silica gel column chromatography (eluent: $\mathrm{PE} / \mathrm{EtOAc}=20 / 1$ to $5 / 1, \mathrm{v} / \mathrm{v}$ ) to yield the corresponding product as a white solid (279 $\mathrm{mg}, 62 \%$ yield).

\subsection{Characterization data of alkene substrates}<smiles>Brc1ccc(/C=C/CN[SnH3])cc1</smiles>

(E)- $\boldsymbol{N}$-(3-(4-Bromophenyl)allyl)-4-methylbenzenesulfonamide (1v): Flash column chromatography (eluent: $\mathrm{PE} / \mathrm{EtOAc}=20 / 1$ to $5 / 1$, v/v) to afford $\mathbf{1 v}$ as a pale yellow solid. $438 \mathrm{mg}, 72 \%$ yield (last step). mp $103.2-103.7{ }^{\circ} \mathrm{C} .{ }^{1} \mathrm{H}$ NMR (400 MHz, $\left.\mathrm{CDCl}_{3}\right) \delta 7.77(\mathrm{~d}, J=8.2 \mathrm{~Hz}, 2 \mathrm{H}), 7.38(\mathrm{~d}, J=8.4 \mathrm{~Hz}, 2 \mathrm{H}), 7.27(\mathrm{~d}, J=8.2 \mathrm{~Hz}, 2 \mathrm{H})$, $7.07(\mathrm{~d}, J=8.4 \mathrm{~Hz}, 2 \mathrm{H}), 6.36(\mathrm{~d}, J=15.8 \mathrm{~Hz}, 1 \mathrm{H}), 5.99(\mathrm{dt}, J=15.8,6.2 \mathrm{~Hz}, 1 \mathrm{H})$, $4.97(\mathrm{t}, J=6.2 \mathrm{~Hz}, 1 \mathrm{H}), 3.72(\mathrm{t}, J=6.2 \mathrm{~Hz}, 2 \mathrm{H}), 2.39(\mathrm{~s}, 3 \mathrm{H}) .{ }^{13} \mathrm{C}$ NMR $(101 \mathrm{MHz}$, $\left.\mathrm{CDCl}_{3}\right) \delta 143.69,137.13,135.21,131.78,131.74,129.86,128.03,127.30,125.18$, 121.77, 45.40, 21.62. HR-ESI-MS $\mathrm{m} / z$ calcd. for $\mathrm{C}_{16} \mathrm{H}_{16} \mathrm{NNaO}_{2} \mathrm{SBr}[\mathrm{M}+\mathrm{Na}]^{+}$: 387.9977, found: 387.9971 .<smiles>Cc1ccc(/C=C/CNS)cc1F</smiles>

(E)- $\boldsymbol{N}$-(3-(3-Fluoro-4-methylphenyl)allyl)-4-methylbenzenesulfonamide

(1x):

Flash column chromatography (eluent: $\mathrm{PE} / \mathrm{EtOAc}=20 / 1$ to $5 / 1, \mathrm{v} / \mathrm{v}$ ) to afford $\mathbf{1 x}$ as a pale yellow solid. $334 \mathrm{mg}, 81 \%$ yield (last step). mp $114.5-115.7{ }^{\circ} \mathrm{C} .{ }^{1} \mathrm{H}$ NMR (400 $\left.\mathrm{MHz} \mathrm{CDCl}_{3}\right) \delta 7.77(\mathrm{~d}, J=8.2 \mathrm{~Hz}, 2 \mathrm{H}), 7.28(\mathrm{~d}, J=8.2 \mathrm{~Hz}, 2 \mathrm{H}), 7.04-7.08(\mathrm{~m}, 1 \mathrm{H})$, $6.91-6.79(\mathrm{~m}, 2 \mathrm{H}), 6.34(\mathrm{~d}, J=15.8 \mathrm{~Hz}, 1 \mathrm{H}), 5.92(\mathrm{dt}, J=15.8,6.3 \mathrm{~Hz}, 1 \mathrm{H}), 4.93$ $(\mathrm{m}, 1 \mathrm{H}), 3.72(\mathrm{t}, J=6.3 \mathrm{~Hz}, 2 \mathrm{H}), 2.40(\mathrm{~s}, 3 \mathrm{H}), 2.23(\mathrm{~s}, 3 \mathrm{H}) .{ }^{13} \mathrm{C} \mathrm{NMR}(101 \mathrm{MHz}$, $\left.\mathrm{CDCl}_{3}\right) \delta 161.45(\mathrm{~d}, J=244.3 \mathrm{~Hz}), 143.68,137.21,136.01(\mathrm{~d}, J=7.7 \mathrm{~Hz}), 131.92$, $131.52(\mathrm{~d}, J=5.5 \mathrm{~Hz}), 129.86,127.31,124.61,122.17(\mathrm{~d}, J=2.9 \mathrm{~Hz}), 112.53(\mathrm{~d}, J=$ 22.9 Hz), 45.39, 21.57, 14.44. ${ }^{19} \mathrm{~F}$ NMR (376 MHz, $\left.\mathrm{CDCl}_{3}\right) \delta-117.74$. HR-ESI-MS $m / z$ calcd. for $\mathrm{C}_{17} \mathrm{H}_{18} \mathrm{NNaO}_{2} \mathrm{FS}[\mathrm{M}+\mathrm{Na}]^{+}: 342.0934$, found: 342.0927 .<smiles>[NH3+]CC=CCCc1ccccc1</smiles>

(E)-4-Methyl- $\boldsymbol{N}$-(5-phenylpent-2-en-1-yl)benzenesulfonamide (1ab): Flash column chromatography (eluent: PE/EtOAc $=20 / 1$ to $5 / 1$, v/v) to afford $\mathbf{1 a b}$ as a white solid. $253 \mathrm{mg}, 69 \%$ yield (last step). mp $52.8-54.0{ }^{\circ} \mathrm{C} .{ }^{1} \mathrm{H} \mathrm{NMR}\left(400 \mathrm{MHz}, \mathrm{CDCl}_{3}\right) \delta 7.74$ $(\mathrm{d}, J=8.2 \mathrm{~Hz}, 2 \mathrm{H}), 7.29$ (d, $J=8.2 \mathrm{~Hz}, 2 \mathrm{H}), 7.25$ (d, $J=7.5 \mathrm{~Hz}, 2 \mathrm{H}), 7.17$ (t, $J=7.5$ 
$\mathrm{Hz}, 1 \mathrm{H}), 7.12(\mathrm{t}, J=6.6 \mathrm{~Hz}, 2 \mathrm{H}), 5.63-5.52(\mathrm{~m}, 1 \mathrm{H}), 5.37-5.26(\mathrm{~m}, 1 \mathrm{H}), 4.60(\mathrm{~s}$, $1 \mathrm{H}), 3.50(\mathrm{t}, J=6.2 \mathrm{~Hz}, 2 \mathrm{H}), 2.62-2.55(\mathrm{~m}, 2 \mathrm{H}), 2.41(\mathrm{~s}, 3 \mathrm{H}), 2.24(\mathrm{dd}, J=14.9,7.2$ $\mathrm{Hz}, 2 \mathrm{H}) .{ }^{13} \mathrm{C} \mathrm{NMR}\left(101 \mathrm{MHz}, \mathrm{CDCl}_{3}\right) \delta 143.49,141.54,137.21,133.98,129.78$, 128.46, 128.42, 127.28, 126.02, 125.27, 45.36, 35.35, 33.90, 21.63. HR-ESI-MS $m / z$ calcd. for $\mathrm{C}_{18} \mathrm{H}_{21} \mathrm{NNaO}_{2} \mathrm{~S}[\mathrm{M}+\mathrm{Na}]^{+}$: 338.1185 , found: 338.1183 .

\section{Synthetic procedures and characterization data of sulfur reagents}

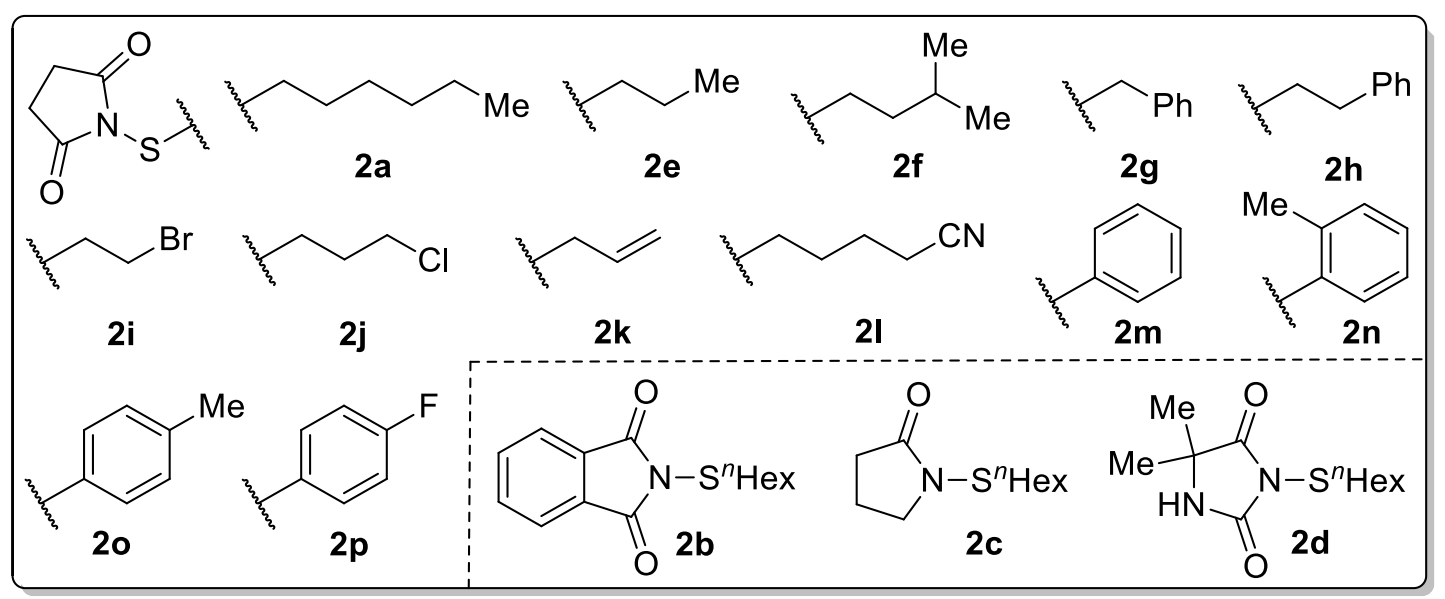

\subsection{Synthesis of $2 \mathrm{a}, 2 \mathrm{~g}-2 \mathrm{~h}$ and $2 \mathrm{~m}-2 \mathrm{p}$}

General procedure for the synthesis of compounds $\mathbf{2 a}, \mathbf{2 g - 2 h}$ and $\mathbf{2 m - 2 p}$ is based on the known procedure. ${ }^{8}$ Compounds $\mathbf{2 m},{ }^{9} \mathbf{2 n},{ }^{10} \mathbf{2 0}{ }^{9}$ and $\mathbf{2} \mathbf{p}^{10}$ have been reported and their spectral data were in accordance with the reported in the literature.

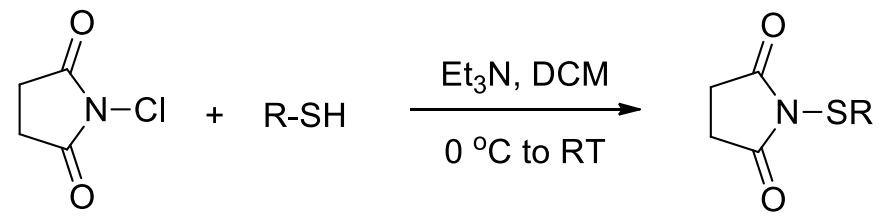

$2 a, 2 g-2 h, 2 m-2 p$

To a solution of NCS $(267 \mathrm{mg}, 2.0 \mathrm{mmol})$ in DCM $(10 \mathrm{~mL})$ was added dropwise thiol or thiophenol (2.0 mmol, 1.0 equiv) under nitrogen atmosphere at $0{ }^{\circ} \mathrm{C}$. After stirring for $5 \mathrm{~min}$, a solution of $\mathrm{Et}_{3} \mathrm{~N}(417 \mu \mathrm{L}, 3.0 \mathrm{mmol})$ in $\mathrm{DCM}(5 \mathrm{~mL})$ was added dropwise over $10 \mathrm{~min}$. The resultant solution was allowed to warm to room temperature and stirred for $2 \mathrm{~h}$. Water $(10 \mathrm{~mL})$ was added. Then, the mixture was extracted with DCM $(15 \mathrm{~mL} \times 3)$ and the combined organic layers were dried over anhydrous $\mathrm{Na}_{2} \mathrm{SO}_{4}$, filtered and concentrated under reduced pressure. The residue was purified by flash silica gel collumn chromotography to give the desired product. 


\subsection{Synthesis of $2 b$ and $2 c$}

General procedure for the synthesis of compounds $\mathbf{2 b}$ and $\mathbf{2 c}$ is based on the known procedure. $^{11}$

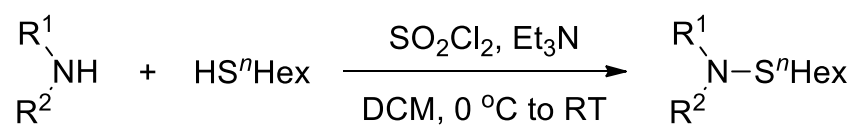

$2 b, 2 c$

To a solution of 1-hexanethiol $(704 \mu \mathrm{L}, 5.0 \mathrm{mmol})$ and catalytic amounts of $\mathrm{Et}_{3} \mathrm{~N}$ (10 $\mu \mathrm{L})$ in dry DCM $(5 \mathrm{~mL})$ was added sulfuryl chloride $(405 \mu \mathrm{L}, 5.0 \mathrm{mmol})$ at $0{ }^{\circ} \mathrm{C}$. After stirring for $30 \mathrm{~min}$ at that temperature, the solution was warmed to room temperature and added by syringe to an ice-chilled suspension of coresponding imide (5.0 mmol, 1.0 equiv) and $\mathrm{Et}_{3} \mathrm{~N}(835 \mu \mathrm{L}, 6.0 \mathrm{mmol})$ in dry DCM $(5 \mathrm{~mL})$. The mixture was stirred for $1.5 \mathrm{~h}$ and then warmed to room temperature. Water $(10 \mathrm{~mL})$ was added. The organic phase was separated by a separatory funnel, washed with water and brine, dried over anhydrous $\mathrm{Na}_{2} \mathrm{SO}_{4}$, filtered, and concentrated under reduced pressure. The residue was purified by flash silica gel collumn chromotography to yield the desired product.

\subsection{Synthesis of $2 d$}

The procedure for the synthesis of compound $\mathbf{2 d}$ is based on the known procedure. ${ }^{8}$<smiles>CC1(C)C(=O)N(Cl)C(=O)N1Cl</smiles><smiles>CCNCCCCC(=O)OCC</smiles><smiles>CC1(C)NC(=O)N(S[GaH])C1=O</smiles>

2d

To a solution of 1,3-dichloro-5,5-dimethylhydantoin (394 mg, $2.0 \mathrm{mmol})$ in DCM (10 $\mathrm{mL}$ ) was added dropwise 1-hexanethiol (564 $\mu \mathrm{L}, 4.0 \mathrm{mmol}$ ) under nitrogen atmosphere at $0{ }^{\circ} \mathrm{C}$. The solution was stirred for $5 \mathrm{~min}$, after which a solution of $\mathrm{Et}_{3} \mathrm{~N}$ $(834 \mu \mathrm{L}, 6.0 \mathrm{mmol})$ in DCM $(5 \mathrm{~mL})$ was added dropwise over $10 \mathrm{~min}$. The resulting solution was allowed to warm to room temperature and stirred for $2 \mathrm{~h}$. Water $(10 \mathrm{~mL})$ was added. The organic phase was separated. The aqueous phase was extracted with DCM $(15 \mathrm{~mL} \times 3)$. The combined organic phases were dried over anhydrous $\mathrm{Na}_{2} \mathrm{SO}_{4}$, filtered and concentrated under reduced pressure. The residue was purified by flash silica gel collumn chromotography to give the desired product.

\subsection{Synthesis of $2 \mathrm{e}-2 \mathrm{f}$ and $2 \mathrm{i}-2 \mathrm{l}$}

General procedure for the synthesis of compounds $\mathbf{2 e - 2 f}$ and $\mathbf{2 i}-\mathbf{2 l}$ is based on the known procedure. ${ }^{12}$ 


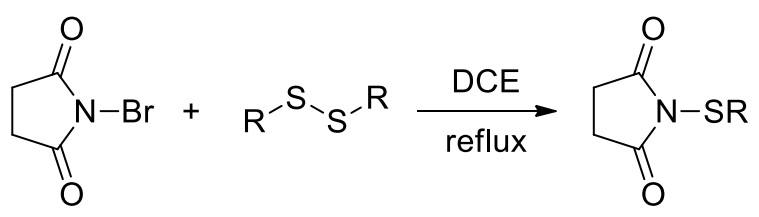

2e-2f, 2i-2l

To a solution of NBS (356 mg, $2.0 \mathrm{mmol})$ in DCE $(5 \mathrm{~mL})$ was added disulfide (2.0 mmol, 1.0 equiv). The solution was refluxed for $2 \mathrm{~h}$, and then cooled to room temperature and concentrated under reduced pressure. The residue was purified by flash silica gel collumn chromotography to give the desired product.

\section{Synthesis of disulfides}

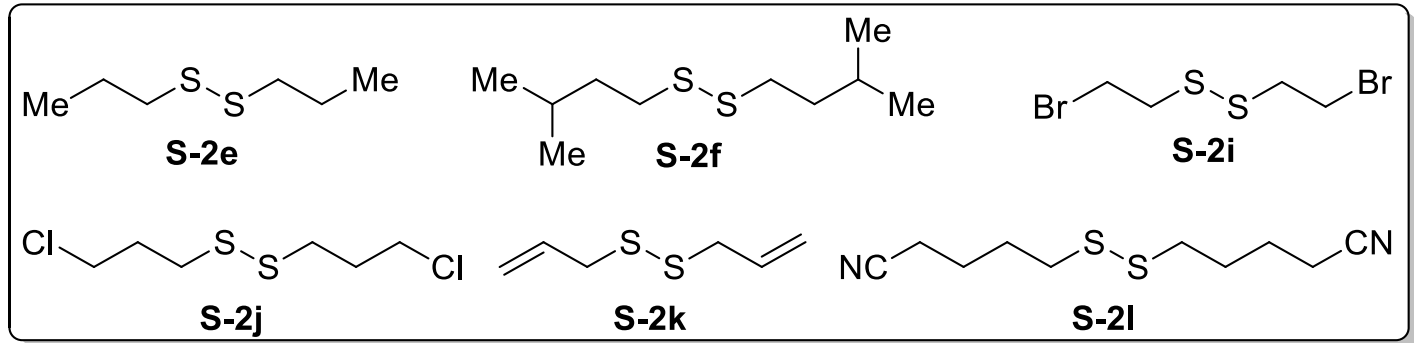

Disulfide S-2e was commercially available. The others were prepared as follows:

Synthesis of S-2f, S-2k and S-21

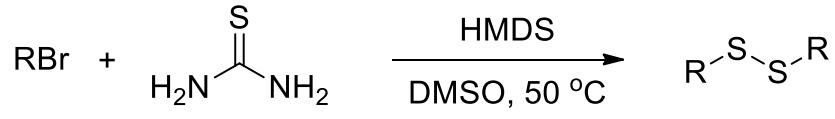

S-2f, S-2k, S-2I

The procedure is based on the literature method. ${ }^{13}$ A solution of bromide $(6.0 \mathrm{mmol}$, 1.0 equiv), thiourea $(548 \mathrm{mg}, 7.2 \mathrm{mmol})$ and hexamethyldisilazane $(1.88 \mathrm{~mL}, 9.0$ mmol) in $10 \mathrm{~mL}$ wet DMSO (drop $120 \mu \mathrm{L} \mathrm{H}_{2} \mathrm{O}$ in $10 \mathrm{~mL}$ DMSO) was heated at $50{ }^{\circ} \mathrm{C}$ for $13 \mathrm{~h}$. Then, the solution was cooled to room temperature. Water $(20 \mathrm{~mL})$ was added. The mixture was extracted with EtOAc $(20 \mathrm{~mL} \times 3)$ and washed with water $(50 \mathrm{~mL} \times 3)$. The combined organic phases were dried over anhydrous $\mathrm{Na}_{2} \mathrm{SO}_{4}$, filtered and concentrated under reduced pressure. The residue was purified by flash silica gel collumn chromotography to afford the desired products.

Synthesis of $\mathbf{S - 2 i}$ 


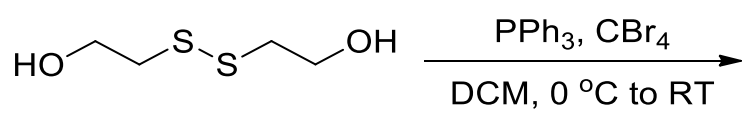<smiles>BrCCSSCCBr</smiles>

To a solution of $\mathrm{PPh}_{3}(1.89 \mathrm{~g}, 7.2 \mathrm{mmol})$ in DCM $(15 \mathrm{~mL})$ was added 2,2'-disulfanediyldiethanol $(359 \mu \mathrm{L}, 3.0 \mathrm{mmol})$ under nitrogen atmosphere at $0{ }^{\circ} \mathrm{C}$. Then, $\mathrm{CBr}_{4}(2.39 \mathrm{~g}, 7.2 \mathrm{mmol})$ was added in one portion. The solution was allowed to warm to room temperature and stirred overnight. Water $(10 \mathrm{~mL})$ was added. Then, the organic phase was separated. The aqueous phase was extracted with DCM $(20 \mathrm{~mL} \times$ 3 ). The combined organic phases were dried over anhydrous $\mathrm{Na}_{2} \mathrm{SO}_{4}$, filtered, and concentrated under reduced pressure. The residue was purified by flash silica gel collumn chromotography (eluent: PE) to give the desired product as a colorless oil (388 mg, 84\% yield).

Synthesis of $\mathbf{S - 2 j}$
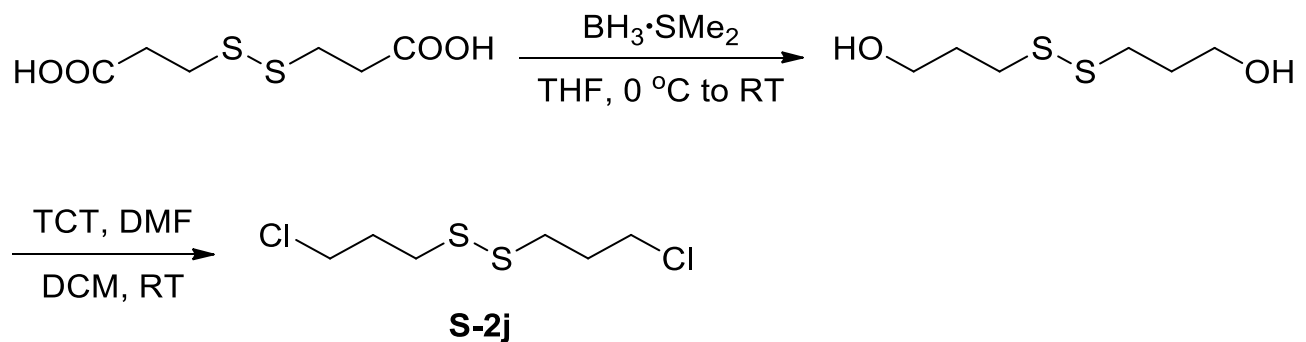

The procedure for the conversion of 3,3'-disulfanediyldipropanoic acid to 3,3'-disulfanediylbis (propan-1-ol) is based on the literature. ${ }^{14}$ To a solution of 3,3'-disulfanediyldipropanoic acid $(631 \mathrm{mg}, 3.0 \mathrm{mmol})$ in THF $(15 \mathrm{~mL})$ was added the borane-methyl sulfide complex (2.0 M in THF, $4.5 \mathrm{~mL}, 9.0 \mathrm{mmol})$ over $30 \mathrm{~min}$ under nitrogen atmosphere at $0{ }^{\circ} \mathrm{C}$. Then, the mixture was stirred for another $30 \mathrm{~min}$ at $0{ }^{\circ} \mathrm{C}$ and $2.5 \mathrm{~h}$ at room temperature. $\mathrm{MeOH}$ was added slowly to dissolve the precipitate and water was added in to quench the reaction. Then, the mixture was extracted with EtOAc $(20 \mathrm{~mL} \times 3)$. The combined organic phases were dried over anhydrous $\mathrm{Na}_{2} \mathrm{SO}_{4}$, filtered and concentrated under reduced pressure. The residue was purified by flash silica gel collumn chromotography (eluent: $\mathrm{PE} / \mathrm{EtOAc}=10 / 1$ to $2 / 1$, v/v) to give the 3,3'-disulfanediylbis(propan-1-ol) as a colorless oil (519 $\mathrm{mg}, 95 \%$ yield).

Chlorination of the 3,3'-disulfanediylbis(propan-1-ol) was conducted by modifying the literature procedure. ${ }^{15}$ Cyanuric chloride $(1.31 \mathrm{~g}, 7.1 \mathrm{mmol})$ was added to DMF (2 $\mathrm{mL})$. The solution was stirred slowly at room temperature. After the formation of a white solid, DCM (15 mL) and the alcohol (519 mg, $2.9 \mathrm{mmol}$ ) was subsequently added. Then, the mixture was stirred at room temperature overnight. Water $(10 \mathrm{~mL})$ was added. The organic phase was separated and subsequently washed with $15 \mathrm{~mL}$ of a saturated solution of $\mathrm{Na}_{2} \mathrm{CO}_{3}, 1 \mathrm{M} \mathrm{HCl}$, and brine. The organic layer was dried over $\mathrm{Na}_{2} \mathrm{SO}_{4}$, filtered and concentrated under reduced pressure. The residue was purified 
by flash silica gel collumn chromotography (eluent: PE) to give the 1,2-bis(3-chloropropyl)disulfane as a colorless oil (503 $\mathrm{mg}, 81 \%$ yield).

\subsection{Characterization data of sulfur reagents}<smiles>CCCCCCSN1C(=O)CCC1=O</smiles>

1-(Hexylthio)pyrrolidine-2,5-dione (2a): Flash column chromatography (eluent: $\mathrm{PE} / \mathrm{EtOAc}=10 / 1$ to $2 / 1, \mathrm{v} / \mathrm{v})$ to afford $2 \mathrm{a}$ as a colorless oil. $370 \mathrm{mg}, 86 \%$ yield. ${ }^{1} \mathrm{H}$ NMR (400 MHz, $\left.\mathrm{CDCl}_{3}\right) \delta 2.92-2.80(\mathrm{~m}, 6 \mathrm{H}), 1.58-1.48(\mathrm{~m}, 2 \mathrm{H}), 1.37-1.44(\mathrm{~m}$, $2 \mathrm{H}), 1.34-1.20(\mathrm{~m}, 4 \mathrm{H}), 0.88(\mathrm{t}, J=7.0 \mathrm{~Hz}, 3 \mathrm{H}) .{ }^{13} \mathrm{C} \mathrm{NMR}\left(101 \mathrm{MHz}, \mathrm{CDCl}_{3}\right) \delta$ 177.25, 37.71, 31.36, 28.69, 28.19, 27.98, 22.52, 14.07. HR-ESI-MS $\mathrm{m} / z$ calcd. for $\mathrm{C}_{10} \mathrm{H}_{17} \mathrm{NNaO}_{2} \mathrm{~S}[\mathrm{M}+\mathrm{Na}]^{+}:$238.0872, found: 238.0871 .<smiles>CCCCCCSN1C(=O)c2ccccc2C1=O</smiles>

2-(Hexylthio)isoindoline-1,3-dione (2b): Flash column chromatography (eluent: $\mathrm{PE} / \mathrm{EtOAc}=15 / 1$ to $5 / 1, \mathrm{v} / \mathrm{v}$ ) to afford $\mathbf{2 b}$ as a white solid. $802 \mathrm{mg}, 61 \%$ yield. $\mathrm{mp}$ $51.0-52.8^{\circ} \mathrm{C} .{ }^{1} \mathrm{H}$ NMR $\left(400 \mathrm{MHz}, \mathrm{CDCl}_{3}\right) \delta 7.93(\mathrm{dd}, J=5.5,3.1 \mathrm{~Hz}, 2 \mathrm{H}), 7.79(\mathrm{dd}$, $J=5.5,3.1 \mathrm{~Hz}, 2 \mathrm{H}), 2.94-2.81(\mathrm{~m}, 2 \mathrm{H}), 1.63-1.54(\mathrm{~m}, 2 \mathrm{H}), 1.49-1.37(\mathrm{~m}, 2 \mathrm{H})$, $1.33-1.20(\mathrm{~m}, 4 \mathrm{H}), 0.86(\mathrm{t}, J=6.9 \mathrm{~Hz}, 3 \mathrm{H}) .{ }^{13} \mathrm{C} \mathrm{NMR}\left(101 \mathrm{MHz}, \mathrm{CDCl}_{3}\right) \delta 168.63$, $134.66,132.17,123.94,38.78,31.40,28.22,22.56,14.08$. HR-ESI-MS $m / z$ calcd. for $\mathrm{C}_{14} \mathrm{H}_{17} \mathrm{NNaO}_{2} \mathrm{~S}[\mathrm{M}+\mathrm{Na}]^{+}:$286.0872, found: 286.0872 .<smiles>CCCCCCSN1CCCC1=O</smiles>

1-(Hexylthio)pyrrolidin-2-one (2c): Flash column chromatography (eluent: $\mathrm{PE} / \mathrm{EtOAc}=10 / 1$ to $3 / 1, \mathrm{v} / \mathrm{v})$ to afford $2 \mathrm{c}$ as a colorless oil. $744 \mathrm{mg}, 74 \%$ yield. ${ }^{1} \mathrm{H}$ NMR (400 MHz, CDCl3) $\delta 3.60(\mathrm{t}, J=7.9 \mathrm{~Hz}, 2 \mathrm{H}), 2.79(\mathrm{t}, J=7.5 \mathrm{~Hz}, 2 \mathrm{H}), 2.47-$ $2.39(\mathrm{~m}, 2 \mathrm{H}), 2.12-2.02(\mathrm{~m}, 2 \mathrm{H}), 1.63-1.52(\mathrm{~m}, 2 \mathrm{H}), 1.47-1.35(\mathrm{~m}, 2 \mathrm{H}), 1.34-$ $1.21(\mathrm{~m}, 4 \mathrm{H}), 0.88(\mathrm{t}, J=6.7 \mathrm{~Hz}, 3 \mathrm{H}) .{ }^{13} \mathrm{C} \mathrm{NMR}\left(101 \mathrm{MHz}, \mathrm{CDCl}_{3}\right) \delta 177.87,52.87$, $37.09,31.43,30.34,28.37,28.20,22.53,18.92,14.04$. HR-ESI-MS $\mathrm{m} / \mathrm{z}$ calcd. for $\mathrm{C}_{10} \mathrm{H}_{20} \mathrm{NOS}[\mathrm{M}+\mathrm{H}]^{+}:$202.1260, found: 202.1254 .

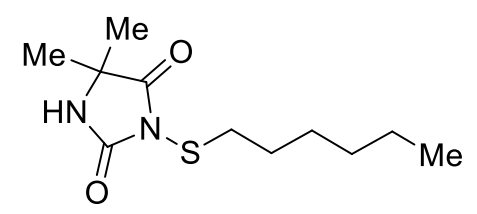


3-(Hexylthio)-5,5-dimethylimidazolidine-2,4-dione (2d): Flash column chromatography (eluent: PE/EtOAc $=10 / 1$ to $3 / 1, \mathrm{v} / \mathrm{v}$ ) to afford $\mathbf{2 d}$ as a colorless oil. $327 \mathrm{mg}, 67 \%$ yield. ${ }^{1} \mathrm{H}$ NMR $\left(400 \mathrm{MHz}, \mathrm{CDCl}_{3}\right) \delta 8.75(\mathrm{~s}, 1 \mathrm{H}), 2.84(\mathrm{t}, J=7.6 \mathrm{~Hz}$, $2 \mathrm{H}), 1.65-1.56(\mathrm{~m}, 2 \mathrm{H}), 1.49-1.36(\mathrm{~m}, 8 \mathrm{H}), 1.36-1.23(\mathrm{~m}, 4 \mathrm{H}), 0.89(\mathrm{t}, J=6.9 \mathrm{~Hz}$, $3 \mathrm{H}) .{ }^{13} \mathrm{C} \mathrm{NMR}\left(101 \mathrm{MHz}, \mathrm{CDCl}_{3}\right) \delta 176.75,157.09,66.55,39.92,31.49,28.55,27.47$, 23.31, 22.60, 14.12. HR-ESI-MS $\mathrm{m} / z$ calcd. for $\mathrm{C}_{11} \mathrm{H}_{21} \mathrm{NO}_{2} \mathrm{~S}[\mathrm{M}+\mathrm{H}]^{+}:$245.1318, found: 245.1313 .<smiles>CCCSN1C(=O)CCC1=O</smiles>

1-(Propylthio)pyrrolidine-2,5-dione (2e): Flash column chromatography (eluent: $\mathrm{PE} / \mathrm{EtOAc}=10 / 1$ to $2 / 1, \mathrm{v} / \mathrm{v}$ ) to afford $2 \mathrm{e}$ as a colorless oil. $301 \mathrm{mg}, 87 \%$ yield. ${ }^{1} \mathrm{H}$ NMR (400 MHz, CDCl3) $\delta 2.87-2.79(\mathrm{~m}, 6 \mathrm{H}), 1.64-1.51(\mathrm{~m}, 2 \mathrm{H}), 1.02(\mathrm{t}, J=7.3$ $\mathrm{Hz}, 3 \mathrm{H}) .{ }^{13} \mathrm{C} \mathrm{NMR}\left(101 \mathrm{MHz}, \mathrm{CDCl}_{3}\right) \delta 177.38,39.78,28.78,21.70,13.18$. HR-ESI-MS $m / z$ calcd. for $\mathrm{C}_{7} \mathrm{H}_{11} \mathrm{NNaO}_{2} \mathrm{~S}[\mathrm{M}+\mathrm{Na}]^{+}:$196.0403, found: 196.0403 .<smiles>CC(C)CCSN1C(=O)CCC1=O</smiles>

1-(Isopentylthio)pyrrolidine-2,5-dione (2f): Flash column chromatography (eluent: $\mathrm{PE} / \mathrm{EtOAc}=10 / 1$ to $2 / 1, \mathrm{v} / \mathrm{v})$ to afford $2 \mathbf{f}$ as a colorless oil. $374 \mathrm{mg}, 93 \%$ yield. ${ }^{1} \mathrm{H}$ NMR $\left(400 \mathrm{MHz}, \mathrm{CDCl}_{3}\right) \delta 2.98-2.82(\mathrm{~m}, 6 \mathrm{H}), 1.80-1.62(\mathrm{~m}, 1 \mathrm{H}), 1.43(\mathrm{dt}, J=$ 13.2, $6.9 \mathrm{~Hz}, 2 \mathrm{H}), 0.89(\mathrm{~d}, J=6.9 \mathrm{~Hz}, 6 \mathrm{H}) .{ }^{13} \mathrm{C} \mathrm{NMR}\left(101 \mathrm{MHz}, \mathrm{CDCl}_{3}\right) \delta 177.26$, $36.60,35.76,28.70,27.33,22.27$. HR-ESI-MS $m / z$ calcd. for $\mathrm{C}_{9} \mathrm{H}_{15} \mathrm{NNaO}_{2} \mathrm{~S}[\mathrm{M}+\mathrm{Na}]^{+}$: 224.0716, found: 224.0708 .<smiles>O=C1CCC(=O)N1SCc1ccccc1</smiles>

1-(Benzylthio)pyrrolidine-2,5-dione (2g): Flash column chromatography (eluent: $\mathrm{PE} / \mathrm{EtOAc}=10 / 1$ to $2 / 1, \mathrm{v} / \mathrm{v}$ ) to afford $\mathbf{2 g}$ as a white solid. $402 \mathrm{mg}, 91 \%$ yield. $\mathrm{mp}$ 123.9 - $124.9{ }^{\circ} \mathrm{C} .{ }^{1} \mathrm{H}$ NMR $\left(400 \mathrm{MHz}, \mathrm{CDCl}_{3}\right) \delta 7.44-7.14(\mathrm{~m}, 5 \mathrm{H}), 4.10(\mathrm{~s}, 2 \mathrm{H})$, $2.63(\mathrm{~s}, 4 \mathrm{H}) .{ }^{13} \mathrm{C}$ NMR $\left(101 \mathrm{MHz}, \mathrm{CDCl}_{3}\right) \delta 176.67,134.01,129.75,128.72,128.18$, 41.10, 28.51. HR-ESI-MS $\mathrm{m} / z$ calcd. for $\mathrm{C}_{11} \mathrm{H}_{11} \mathrm{NNaO}_{2} \mathrm{~S}[\mathrm{M}+\mathrm{Na}]^{+}: 244.0403$, found: 224.0403 .<smiles>O=C1CCC(=O)N1SCCc1ccccc1</smiles> 
1-(Phenethylthio)pyrrolidine-2,5-dione (2h): Flash column chromatography (eluent: $\mathrm{PE} / \mathrm{EtOAc}=10 / 1$ to $2 / 1, \mathrm{v} / \mathrm{v})$ to afford $\mathbf{2 h}$ as a white solid. $414 \mathrm{mg}, 88 \%$ yield. $\mathrm{mp}$ 88.5 - 89.4 ${ }^{\circ} \mathrm{C} .{ }^{1} \mathrm{H}$ NMR (400 MHz, $\left.\mathrm{CDCl}_{3}\right) \delta 7.32-7.25(\mathrm{~m}, 2 \mathrm{H}), 7.23-7.16(\mathrm{~m}$, $3 \mathrm{H}), 3.19(\mathrm{t}, J=7.3 \mathrm{~Hz}, 2 \mathrm{H}), 2.96(\mathrm{t}, J=7.3 \mathrm{~Hz}, 2 \mathrm{H}), 2.63(\mathrm{~s}, 4 \mathrm{H}) .{ }^{13} \mathrm{C}$ NMR $(101$ $\left.\mathrm{MHz}, \mathrm{CDCl}_{3}\right) \delta 177.15,139.10,128.67,128.50,126.72,37.67,35.42,28.65$. HR-ESI-MS $m / z$ calcd. for $\mathrm{C}_{12} \mathrm{H}_{13} \mathrm{NNaO}_{2} \mathrm{~S}[\mathrm{M}+\mathrm{Na}]^{+}: 258.0559$, found: 258.0554 .<smiles>O=C1CCC(=O)N1SCCBr</smiles>

1-((2-Bromoethyl)thio)pyrrolidine-2,5-dione (2i): Flash column chromatography (eluent: PE/EtOAc $=5 / 1$ to $1 / 1, \mathrm{v} / \mathrm{v}$ ) to afford $2 \mathbf{i}$ as a yellow oil. $279 \mathrm{mg}, 59 \%$ yield. ${ }^{1} \mathrm{H}$ NMR (400 MHz, $\mathrm{CDCl}_{3}$ ) $\delta 3.53(\mathrm{t}, J=8.1 \mathrm{~Hz}, 2 \mathrm{H}), 3.24(\mathrm{t}, J=8.1 \mathrm{~Hz}, 2 \mathrm{H}), 2.88$ (s, 4H). ${ }^{13} \mathrm{C} \mathrm{NMR}\left(101 \mathrm{MHz}, \mathrm{CDCl}_{3}\right) \delta 177.03,40.07,29.23,28.73$. HR-ESI-MS $\mathrm{m} / \mathrm{z}$ calcd. for $\mathrm{C}_{6} \mathrm{H}_{8} \mathrm{NBrNaO}_{2} \mathrm{~S}[\mathrm{M}+\mathrm{Na}]^{+}: 259.9351$, found: 259.9352 .<smiles>O=C1CCC(=O)N1SCCCCl</smiles>

1-((3-Chloropropyl)thio)pyrrolidine-2,5-dione (2j): Flash column chromatography (eluent: $\mathrm{PE} / \mathrm{EtOAc}=5 / 1$ to $1 / 1, \mathrm{v} / \mathrm{v}$ ) to afford $\mathbf{2 j}$ as a colorless oil. $257 \mathrm{mg}, 62 \%$ yield. ${ }^{1} \mathrm{H}$ NMR (400 MHz, $\left.\mathrm{CDCl}_{3}\right) \delta 3.77(\mathrm{t}, J=6.0 \mathrm{~Hz}, 2 \mathrm{H}), 3.03(\mathrm{t}, J=6.7 \mathrm{~Hz}, 2 \mathrm{H}), 2.86$ (s, 4H), $2.05-1.94(\mathrm{~m}, 2 \mathrm{H}) .{ }^{13} \mathrm{C}$ NMR $\left(101 \mathrm{MHz}, \mathrm{CDCl}_{3}\right) \delta 177.15,42.79,34.89$, 30.84, 28.60. HR-ESI-MS $m / z$ calcd. for $\mathrm{C}_{7} \mathrm{H}_{10} \mathrm{NClNaO}_{2} \mathrm{~S}[\mathrm{M}+\mathrm{Na}]^{+}: 230.0013$, found: 230.0014 .<smiles>C=CCSN1C(=O)CCC1=O</smiles>

1-(Allylthio)pyrrolidine-2,5-dione (2k): Flash column chromatography (eluent: $\mathrm{PE} / \mathrm{EtOAc}=5 / 1$ to $1 / 1, \mathrm{v} / \mathrm{v})$ to afford $\mathbf{2 k}$ as a yellow oil. $191 \mathrm{mg}, 56 \%$ yield. ${ }^{1} \mathrm{H}$ NMR $\left(400 \mathrm{MHz}, \mathrm{CDCl}_{3}\right) \delta 5.91-5.72(\mathrm{~m}, 1 \mathrm{H}), 5.06(\mathrm{dd}, J=33.0,13.3 \mathrm{~Hz}, 2 \mathrm{H})$, $3.51(\mathrm{~d}, J=7.5 \mathrm{~Hz}, 2 \mathrm{H}), 2.83(\mathrm{~s}, 4 \mathrm{H}) .{ }^{13} \mathrm{C} \mathrm{NMR}\left(101 \mathrm{MHz}, \mathrm{CDCl}_{3}\right) \delta 177.00,131.53$, 119.64, 40.35, 28.57. HR-ESI-MS $m / z$ calcd. for $\mathrm{C}_{7} \mathrm{H}_{10} \mathrm{NO}_{2} \mathrm{~S}[\mathrm{M}+\mathrm{H}]^{+}:$172.0427, found: 172.0427 .<smiles>N#CCCCCSN1C(=O)CCC1=O</smiles> 

mg, $75 \%$ yield. ${ }^{1} \mathrm{H}$ NMR $\left(400 \mathrm{MHz}, \mathrm{CDCl}_{3}\right) \delta 2.89(\mathrm{t}, J=7.0 \mathrm{~Hz}, 2 \mathrm{H}), 2.86(\mathrm{~s}, 4 \mathrm{H})$, $2.41(\mathrm{t}, J=7.0 \mathrm{~Hz}, 2 \mathrm{H}), 1.93-1.82(\mathrm{~m}, 2 \mathrm{H}), 1.75-1.62(\mathrm{~m}, 2 \mathrm{H}) .{ }^{13} \mathrm{C}$ NMR $(101$ $\left.\mathrm{MHz}, \mathrm{CDCl}_{3}\right) \delta 177.26,119.26,36.63,28.61,26.73,23.49,16.71$. HR-ESI-MS $\mathrm{m} / z$ calcd. for $\mathrm{C}_{9} \mathrm{H}_{12} \mathrm{~N}_{2} \mathrm{NaO}_{2} \mathrm{~S}[\mathrm{M}+\mathrm{Na}]^{+}:$235.0512, found: 235.0516 .

\section{Synthetic procedures and characterization data of catalysts}

Catalyst C1 and C5-C14 were synthesized as reported previously. ${ }^{16}$ The others were synthesized as follows:

\subsection{Synthesis of $\mathrm{C} 2$}<smiles>NC1c2ccccc2CC1[Se]c1ccccc1</smiles><smiles>[NH3+]C1c2ccccc2CC1[SeH]c1ccccc1</smiles>

C2

Amine was synthesized as reported previously. ${ }^{16}$ To a solution of amine (289 mg, 1.0 mmol) in DCM $(8 \mathrm{~mL})$ were added triethylamine $(209 \mu \mathrm{L}, 1.5 \mathrm{mmol})$ and tosyl chloride $(210 \mathrm{mg}, 1.1 \mathrm{mmol})$ at $0{ }^{\circ} \mathrm{C}$. The solution was stirred for $4 \mathrm{~h}$ at room temperature, and then diluted with water $(5 \mathrm{~mL})$ and extracted with DCM $(10 \mathrm{~mL} \times 3)$. Organic extracts were dried over $\mathrm{Na}_{2} \mathrm{SO}_{4}$ and filtered. After concentration, the residue was purified by flash silica gel column chromatography to yield the corresponding product.

\subsection{Synthesis of $\mathrm{C3}$}<smiles>NC1c2ccccc2CC1[SeH]c1ccccc1</smiles><smiles>[N+]=S(=O)(O)C1c2ccccc2CC1[Se]c1ccccc1</smiles>

C3

To a solution of amine $(289 \mathrm{mg}, 1.0 \mathrm{mmol})$ in DCM $(8 \mathrm{~mL})$ were added triethylamine (209 $\mu \mathrm{L}, 1.5 \mathrm{mmol})$ and 4-nitrobenzenesulfonyl chloride $(244 \mathrm{mg}, 1.1 \mathrm{mmol})$ at $0{ }^{\circ} \mathrm{C}$. The solution was stirred for $4 \mathrm{~h}$ at room temperature, and then diluted with water $(5$ $\mathrm{mL})$ and extracted with $\mathrm{DCM}(10 \mathrm{~mL} \times 3)$. Organic extracts were dried over $\mathrm{Na}_{2} \mathrm{SO}_{4}$ and filtered. After concentration, the residue was purified by flash silica gel column chromatography to yield the corresponding product. 


\subsection{Synthesis of C4}<smiles>N[C@@H]1c2ccccc2C[C@H]1Sc1ccccc1</smiles><smiles>[R16]C[C@H]1c2ccccc2C[C@H]1[SeH]</smiles>

C4

To a solution of amine (289 $\mathrm{mg}, 1.0 \mathrm{mmol})$ in DCM $(8 \mathrm{~mL})$ were added triethylamine $(209 \mu \mathrm{L}, 1.5 \mathrm{mmol})$ and benzoyl chloride $(129 \mu \mathrm{L}, 1.1 \mathrm{mmol})$ at $0{ }^{\circ} \mathrm{C}$. The solution was stirred for $4 \mathrm{~h}$ at room temperature, and then diluted with water $(5 \mathrm{~mL})$ and extracted with DCM $(10 \mathrm{~mL} \times 3)$. Organic extracts were dried over $\mathrm{Na}_{2} \mathrm{SO}_{4}$ and filtered. After concentration, the residue was purified by flash silica gel column chromatography to yield the corresponding product.

\subsection{Characterization data of catalysts}<smiles>[NH3+][C@@H]1c2ccccc2C[C@H]1[SeH]</smiles>

4-Methyl- $N$-((1S,2S)-2-(phenylselanyl)-2,3-dihydro-1H-inden-1-yl)benzenesulfon amide (C2): Flash column chromatography (eluent: PE/EtOAc $=15 / 1$ to $5 / 1$, v/v) to afford C2 as a white solid. $403 \mathrm{mg}, 91 \%$ yield. $\mathrm{mp} 117.2-118.4{ }^{\circ} \mathrm{C} .[\alpha]^{25} \mathrm{D}=6.6(\mathrm{c}=$ 0.2, $\left.\mathrm{CHCl}_{3}\right) .{ }^{1} \mathrm{H}$ NMR $\left(400 \mathrm{MHz}, \mathrm{CDCl}_{3}\right) \delta 7.69(\mathrm{~d}, J=8.3 \mathrm{~Hz}, 2 \mathrm{H}), 7.52-7.46(\mathrm{~m}$, $2 \mathrm{H}), 7.34-7.24(\mathrm{~m}, 5 \mathrm{H}), 7.22-7.18(\mathrm{~m}, 1 \mathrm{H}), 7.17-7.09(\mathrm{~m}, 2 \mathrm{H}), 7.01(\mathrm{~d}, J=8.0$ $\mathrm{Hz}, 1 \mathrm{H}), 4.79-4.66(\mathrm{~m}, 2 \mathrm{H}), 3.80(\mathrm{dt}, J=7.2,5.2 \mathrm{~Hz}, 1 \mathrm{H}), 3.39$ (dd, $J=16.7,7.2 \mathrm{~Hz}$, $1 \mathrm{H}), 2.90(\mathrm{dd}, J=16.7,5.2 \mathrm{~Hz}, 1 \mathrm{H}), 2.45(\mathrm{~s}, 3 \mathrm{H}) .{ }^{13} \mathrm{C} \mathrm{NMR}\left(101 \mathrm{MHz}, \mathrm{CDCl}_{3}\right) \delta$ $143.72,141.80,140.82,137.65,135.39,129.86,129.22$, 129.00, 128.30, 128.08, 127.61, 127.54, 124.93, 124.87, 64.33, 47.19, 38.18, 21.74. HR-ESI-MS m/z calcd. for $\mathrm{C}_{22} \mathrm{H}_{21} \mathrm{NNaO}_{2} \mathrm{SSe}[\mathrm{M}+\mathrm{Na}]^{+}: 466.0350$, found: 466.0344 .

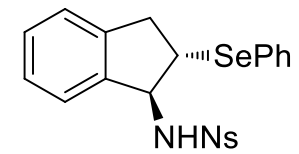

\section{4-Nitro- $N$-((1S,2S)-2-(phenylselanyl)-2,3-dihydro- $1 H$-inden-1-yl)benzenesulfona} mide (C3): Flash column chromatography (eluent: $\mathrm{PE} / \mathrm{EtOAc}=15 / 1$ to $5 / 1$, v/v) to afford $\mathbf{C 3}$ as a pale green solid. $408 \mathrm{mg}, 86 \%$ yield. mp $134.2-135.3{ }^{\circ} \mathrm{C} .[\alpha]^{25} \mathrm{D}=$ $11.0\left(\mathrm{c}=0.2, \mathrm{CHCl}_{3}\right) .{ }^{1} \mathrm{H} \mathrm{NMR}\left(400 \mathrm{MHz}, \mathrm{CDCl}_{3}\right) \delta 8.33-8.24(\mathrm{~m}, 2 \mathrm{H}), 7.97-7.90$ $(\mathrm{m}, 2 \mathrm{H}), 7.47-7.42(\mathrm{~m}, 2 \mathrm{H}), 7.35-7.20(\mathrm{~m}, 4 \mathrm{H}), 7.20-7.08(\mathrm{~m}, 3 \mathrm{H}), 5.06(\mathrm{~d}, J=$ $8.1 \mathrm{~Hz}, 1 \mathrm{H}), 4.80(\mathrm{dd}, J=8.1,5.8 \mathrm{~Hz}, 1 \mathrm{H}), 3.73(\mathrm{dt}, J=7.5,6.1 \mathrm{~Hz}, 1 \mathrm{H}), 3.39$ (dd, $J$ $=16.7,7.5 \mathrm{~Hz}, 1 \mathrm{H}), 2.93(\mathrm{dd}, J=16.7,6.1 \mathrm{~Hz}, 1 \mathrm{H}) .{ }^{13} \mathrm{C} \mathrm{NMR}\left(101 \mathrm{MHz}, \mathrm{CDCl}_{3}\right) \delta$ $150.14,146.51,141.62,140.18,135.05,129.39$, 129.30, 128.62, 128.28, 128.10, 127.78, 125.01, 124.70, 124.42, 64.94, 46.97, 38.22. HR-ESI-MS $\mathrm{m} / \mathrm{z}$ calcd. for $\mathrm{C}_{21} \mathrm{H}_{18} \mathrm{~N}_{2} \mathrm{NaO}_{4} \mathrm{SSe}[\mathrm{M}+\mathrm{Na}]^{+}:$497.0045, found: 497.0041 . 
<smiles>[R16]C1c2ccccc2CC1[SeH]c1ccccc1</smiles>

$\boldsymbol{N}$-((1S,2S)-2-(Phenylselanyl)-2,3-dihydro-1H-inden-1-yl)benzamide (C4): Flash column chromatography (eluent: $\mathrm{PE} / \mathrm{EtOAc}=15 / 1$ to $5 / 1, \mathrm{v} / \mathrm{v}$ ) to afford $\mathbf{C 4}$ as a white solid. $377 \mathrm{mg}, 96 \%$ yield. $\mathrm{mp} 172.1-173.3{ }^{\circ} \mathrm{C} .[\alpha]^{25} \mathrm{D}=104.0\left(\mathrm{c}=0.2, \mathrm{CHCl}_{3}\right) .{ }^{1} \mathrm{H}$ NMR $\left(400 \mathrm{MHz}, \mathrm{CDCl}_{3}\right) \delta 7.76-7.62(\mathrm{~m}, 4 \mathrm{H}), 7.49(\mathrm{t}, J=7.4 \mathrm{~Hz}, 1 \mathrm{H}), 7.40(\mathrm{t}, J=$ $7.6 \mathrm{~Hz}, 2 \mathrm{H}), 7.27-7.14(\mathrm{~m}, 6 \mathrm{H}), 6.32(\mathrm{~d}, J=8.2 \mathrm{~Hz}, 1 \mathrm{H}), 5.72(\mathrm{t}, J=8.4 \mathrm{~Hz}, 1 \mathrm{H})$, $3.78(\mathrm{dd}, J=16.1,8.2 \mathrm{~Hz}, 1 \mathrm{H}), 3.38(\mathrm{dd}, J=16.1,7.7 \mathrm{~Hz}, 1 \mathrm{H}), 3.04(\mathrm{dd}, J=16.1,8.9$ $\mathrm{Hz}, 1 \mathrm{H}) .{ }^{13} \mathrm{C} \mathrm{NMR}\left(101 \mathrm{MHz}, \mathrm{CDCl}_{3}\right) \delta 167.50,142.34,141.60,135.80,134.38$, $131.78,129.25,128.69$, 128.44, 128.15, 128.12, 127.43, 127.17, 124.65, 124.09, 60.91, 47.92, 38.47. HR-ESI-MS $\mathrm{m} / z$ calcd. for $\mathrm{C}_{22} \mathrm{H}_{20} \mathrm{NOSe}[\mathrm{M}+\mathrm{H}]^{+}:$394.0705, found: 394.0706 .<smiles>CCCC1c2ccccc2CC1[18O]c1cccc(OC)c1</smiles>

\section{1,1,1-Trifluoro- $N$-((1S,2S)-2-((3-methoxyphenyl)selanyl)-2,3-dihydro-1H-inden-1} -yl)methanesulfonamide (C9): Flash column chromatography (eluent: PE/EtOAc = 30/1 to 10/1, v/v) to afford C9 as a white solid. $144 \mathrm{mg}, 32 \%$ yield. mp $103.6-105.3$ ${ }^{\circ} \mathrm{C} .[\alpha]^{25} \mathrm{D}=67.2\left(\mathrm{c}=0.2, \mathrm{CHCl}_{3}\right) .{ }^{1} \mathrm{H} \mathrm{NMR}\left(400 \mathrm{MHz}, \mathrm{CDCl}_{3}\right) \delta 7.38-7.33(\mathrm{~m}, 1 \mathrm{H})$, $7.31-7.27(\mathrm{~m}, 2 \mathrm{H}), 7.22-7.17(\mathrm{~m}, 4 \mathrm{H}), 6.89-6.83(\mathrm{~m}, 1 \mathrm{H}), 5.08-5.00(\mathrm{~m}, 1 \mathrm{H})$, $4.97(\mathrm{~s}, 1 \mathrm{H}), 3.83-3.71(\mathrm{~m}, 4 \mathrm{H}), 3.44(\mathrm{dd}, J=16.7,7.4 \mathrm{~Hz}, 1 \mathrm{H}), 3.01(\mathrm{dd}, J=16.7$, $6.5 \mathrm{~Hz}, 1 \mathrm{H}) .{ }^{13} \mathrm{C} \mathrm{NMR}\left(101 \mathrm{MHz}, \mathrm{CDCl}_{3}\right) \delta 159.92,141.67,139.29,130.15,129.67$, 128.28, 128.02, 125.18, 124.57, 124.42, $120.87,119.97$ (q, $J=320.4 \mathrm{~Hz}$ ), 114.76, 65.99, 55.50, 47.74, 38.14. ${ }^{19} \mathrm{~F}$ NMR $\left(376 \mathrm{MHz}, \mathrm{CDCl}_{3}\right) \delta-76.98$. HR-ESI-MS m/z calcd. for $\mathrm{C}_{17} \mathrm{H}_{16} \mathrm{NNaO}_{3} \mathrm{~F}_{3} \mathrm{SSe}[\mathrm{M}+\mathrm{Na}]^{+}$: 473.9860, found: 473.9866 .<smiles>CCCC1c2ccccc2CC1[Hg]c1cc(OC)cc(OC)c1</smiles>

$N$-((1S,2S)-2-((3,5-Dimethoxyphenyl)selanyl)-2,3-dihydro-1H-inden-1-yl)-1,1,1-tr ifluoromethanesulfonamide (C10): Flash column chromatography (eluent: $\mathrm{PE} / \mathrm{EtOAc}=30 / 1$ to $10 / 1, \mathrm{v} / \mathrm{v})$ to afford $\mathbf{C 1 0}$ as a white solid. $84.7 \mathrm{mg}, 26 \%$ yield. $\mathrm{mp}$ $124.7-125.3{ }^{\circ} \mathrm{C} .[\alpha]^{25} \mathrm{D}=68.1\left(\mathrm{c}=0.2, \mathrm{CHCl}_{3}\right) .{ }^{1} \mathrm{H} \mathrm{NMR}\left(400 \mathrm{MHz}, \mathrm{CDCl}_{3}\right) \delta 7.39$ $-7.34(\mathrm{~m}, 1 \mathrm{H}), 7.32-7.27(\mathrm{~m}, 2 \mathrm{H}), 7.23-7.18(\mathrm{~m}, 1 \mathrm{H}), 6.78(\mathrm{~d}, J=2.3 \mathrm{~Hz}, 2 \mathrm{H})$, $6.40(\mathrm{t}, J=2.3 \mathrm{~Hz}, 1 \mathrm{H}), 5.05(\mathrm{~d}, J=6.0 \mathrm{~Hz}, 1 \mathrm{H}), 4.99(\mathrm{~s}, 1 \mathrm{H}), 3.84-3.70(\mathrm{~m}, 7 \mathrm{H})$, $3.44(\mathrm{dd}, J=16.7,7.4 \mathrm{~Hz}, 1 \mathrm{H}), 3.03(\mathrm{dd}, J=16.7,6.7 \mathrm{~Hz}, 1 \mathrm{H}) .{ }^{13} \mathrm{C} \mathrm{NMR}(101 \mathrm{MHz}$, $\left.\mathrm{CDCl}_{3}\right) \delta 160.92,141.63,139.30,129.61,128.60,127.98,125.15,124.47,119.76(\mathrm{q}$, 
$J=321.0 \mathrm{~Hz}) .113 .47,101.26,65.88,55.60,47.77,38.12 .{ }^{19} \mathrm{~F}$ NMR $(376 \mathrm{MHz}$, $\left.\mathrm{CDCl}_{3}\right) \delta$-76.96. HR-ESI-MS $m / z$ calcd. for $\mathrm{C}_{18} \mathrm{H}_{17} \mathrm{NO}_{4} \mathrm{~F}_{3} \mathrm{SSe}[\mathrm{M}-\mathrm{H}]^{-}:$480.0001, found: 479.9987.<smiles>CCNC1c2ccccc2C[C@H]1Oc1cc(C(C)(C)C)cc(Br)c1OCC</smiles>

$N$-((1S,2S)-2-((3,5-Di-tert-butyl-2-ethoxyphenyl)selanyl)-2,3-dihydro-1H-inden-1yl)-1,1,1-trifluoromethanesulfonamide (C14): Flash column chromatography (eluent: $\mathrm{PE} / \mathrm{EtOAc}=50 / 1$ to $30 / 1, \mathrm{v} / \mathrm{v}$ ) to afford $\mathbf{C 1 4}$ as a white solid. $124 \mathrm{mg}, 21 \%$ yield. mp $115.2-116.6{ }^{\circ} \mathrm{C} .[\alpha]^{25} \mathrm{D}=57.5\left(\mathrm{c}=0.2, \mathrm{CHCl}_{3}\right) .{ }^{1} \mathrm{H}$ NMR $(400 \mathrm{MHz}$, $\left.\mathrm{CDCl}_{3}\right) \delta 7.44(\mathrm{~d}, J=2.4 \mathrm{~Hz}, 1 \mathrm{H}), 7.40-7.36(\mathrm{~m}, 1 \mathrm{H}), 7.32(\mathrm{~d}, J=2.4 \mathrm{~Hz}, 1 \mathrm{H}), 7.30$ - $7.25(\mathrm{~m}, 2 \mathrm{H}), 7.20-7.17(\mathrm{~m}, 1 \mathrm{H}), 5.06(\mathrm{~d}, J=4.1 \mathrm{~Hz}, 1 \mathrm{H}), 4.93(\mathrm{~s}, 1 \mathrm{H}), 4.17-$ $4.01(\mathrm{~m}, 3 \mathrm{H}), 3.93(\mathrm{dt}, J=7.4,5.6 \mathrm{~Hz}, 1 \mathrm{H}), 3.40(\mathrm{dd}, J=17.0,7.4 \mathrm{~Hz}, 1 \mathrm{H}), 2.99(\mathrm{dd}$, $J=17.0,5.8 \mathrm{~Hz}, 1 \mathrm{H}), 1.41-1.33(\mathrm{~m}, 13 \mathrm{H}), 1.29(\mathrm{~s}, 9 \mathrm{H}) .{ }^{13} \mathrm{C} \mathrm{NMR}\left(101 \mathrm{MHz}, \mathrm{CDCl}_{3}\right)$ $\delta 156.75,146.52,143.06,141.95,139.65,132.54,129.46,127.82,125.67,125.10$, 124.76, 122.68, 119.75 (q, $J=321.0 \mathrm{~Hz}), 69.77,66.23,46.55,38.50,35.74,34.64$, 31.54, 31.18, 15.34. ${ }^{19} \mathrm{~F}$ NMR $\left(376 \mathrm{MHz}, \mathrm{CDCl}_{3}\right) \delta-77.10$. HR-ESI-MS $\mathrm{m} / z$ calcd. for $\mathrm{C}_{26} \mathrm{H}_{34} \mathrm{NNaO}_{3} \mathrm{~F}_{3} \mathrm{SSe}[\mathrm{M}+\mathrm{Na}]^{+}$: 600.1269 , found: 600.1278 .

\section{Catalytic enantioselective difunctionalization of $\mathrm{N}$-allyl sulfonamides and characterization data of difunctionalization products}

\subsection{Azidothiolation of $N$-allyl sulfonamides}

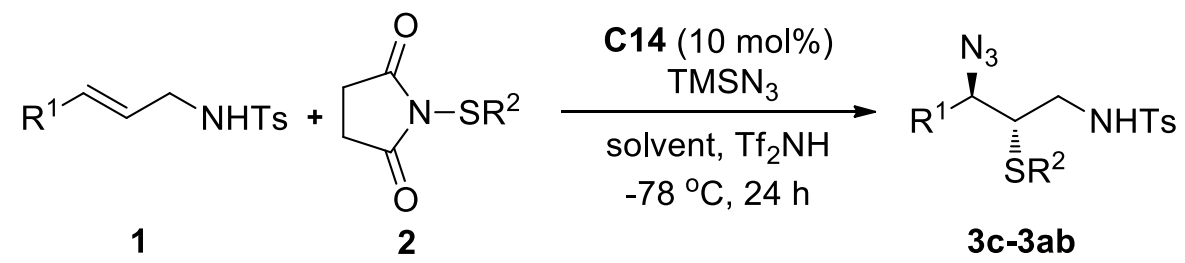

General procedure for the synthesis of $\mathbf{3 c}-\mathbf{3 y}$

To a 10 -mL Schlenk flask charged with a magnetic stirring bar were added $N$-allyl sulfonamide ( $0.1 \mathrm{mmol}, 1.0$ equiv), sulfur reagent $(0.15 \mathrm{mmol}, 1.5$ equiv), catalyst C14 (5.8 $\mathrm{mg}, 10 \mathrm{~mol} \%$ ) and $\mathrm{CH}_{2} \mathrm{Cl}_{2} /$ toluene $(2 \mathrm{~mL} / 2 \mathrm{~mL})$ subsequently. The flask was placed into a cooling bath at $-78{ }^{\circ} \mathrm{C}$. $\mathrm{Tf}_{2} \mathrm{NH}(56.2 \mathrm{mg}, 0.2 \mathrm{mmol})$ was added after the solution was stirred for about $5 \mathrm{~min}$ at $-78{ }^{\circ} \mathrm{C}$. After another $5 \mathrm{~min}, \mathrm{TMSN}_{3}(26.3$ $\mu \mathrm{L}, 0.2 \mathrm{mmol}$ ) was added. The resulting solution was stirred at $-78{ }^{\circ} \mathrm{C}$ for $24 \mathrm{~h}$. and 
then quenched with $5 \% \mathrm{NaHCO}_{3}$ aqueous solution $(4 \mathrm{~mL})$ and extracted with DCM (5 $\mathrm{mL} \times 3$ ). The organic layers were dried over $\mathrm{Na}_{2} \mathrm{SO}_{4}$, filtered, and concentrated under reduced pressure. The residue was purified by flash silica gel column chromatography to yield the corresponding product.

General procedure for the synthesis of $\mathbf{3 z - 3} \mathbf{3 a b}$

To a $10-\mathrm{mL}$ Schlenk flask charged with a magnetic stirring bar were added $N$-allyl sulfonamide ( $0.1 \mathrm{mmol}, 1.0$ equiv), sulfur reagent ( $0.15 \mathrm{mmol}, 1.5$ equiv), catalyst C14 (5.8 mg, $10 \mathrm{~mol} \%$ ) and toluene (4 mL) subsequently. The flask was placed into a cooling bath at $-78{ }^{\circ} \mathrm{C}$. $\mathrm{Tf}_{2} \mathrm{NH}(56.2 \mathrm{mg}, 0.2 \mathrm{mmol})$ was added after the solution was stirred for about $5 \mathrm{~min}$ at $-78{ }^{\circ} \mathrm{C}$. After another $5 \mathrm{~min}, \mathrm{TMSN}_{3}(26.3 \mu \mathrm{L}, 0.2 \mathrm{mmol})$ was added. The resulting solution was stirred at $-78{ }^{\circ} \mathrm{C}$ for $24 \mathrm{~h}$, and then quenched with $5 \% \mathrm{NaHCO}_{3}$ aqueous solution $(4 \mathrm{~mL})$ and extracted with EtOAc $(5 \mathrm{~mL} \times 3)$. The organic layers were dried over $\mathrm{Na}_{2} \mathrm{SO}_{4}$, filtered, and concentrated under reduced pressure. The residue was purified by flash silica gel column chromatography to yield the corresponding product.

Gram-scale experiment

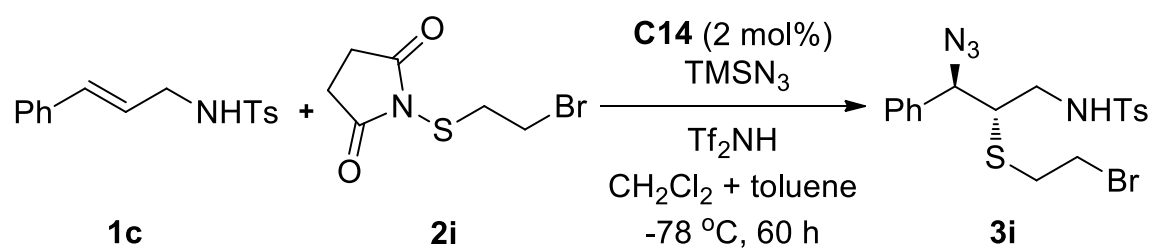

To a 250-mL Schlenk flask charged with a magnetic stirring bar were added $N$-cinnamyl-4-methylbenzenesulfonamide (1.00 g, $3.5 \mathrm{mmol})$, sulfur reagent $2 \mathbf{i}$ (1.24 g, $5.3 \mathrm{mmol})$, catalyst $\mathbf{C 1 4}(40.4 \mathrm{mg}, 2 \mathrm{~mol} \%)$ and $\mathrm{CH}_{2} \mathrm{Cl}_{2} /$ toluene $(70 \mathrm{~mL} / 70 \mathrm{~mL})$ subsequently. The flask was placed into a cooling bath at $-78{ }^{\circ} \mathrm{C} . \mathrm{Tf}_{2} \mathrm{NH}(2.00 \mathrm{~g}, 7.0$ mmol) was added in four portions after the solution was stirred for about 5 min at -78 ${ }^{\circ} \mathrm{C}$. After another $5 \mathrm{~min}, \mathrm{TMSN}_{3}(910 \mu \mathrm{L}, 7.0 \mathrm{mmol})$ was added. The resulting solution was stirred at $-78{ }^{\circ} \mathrm{C}$ for $60 \mathrm{~h}$, and then quenched with $5 \% \mathrm{NaHCO}_{3}$ aqueous solution $(50 \mathrm{~mL})$ and extracted with $\mathrm{DCM}(100 \mathrm{~mL} \times 3)$. The organic layers were dried over $\mathrm{Na}_{2} \mathrm{SO}_{4}$, filtered, and concentrated under reduced pressure. The residue was purified by flash silica gel column chromatography to yield the corresponding product (1.24 g, 76\% yield and 94\% ee).

\subsection{Oxythiolation of $N$-allyl sulfonamides}

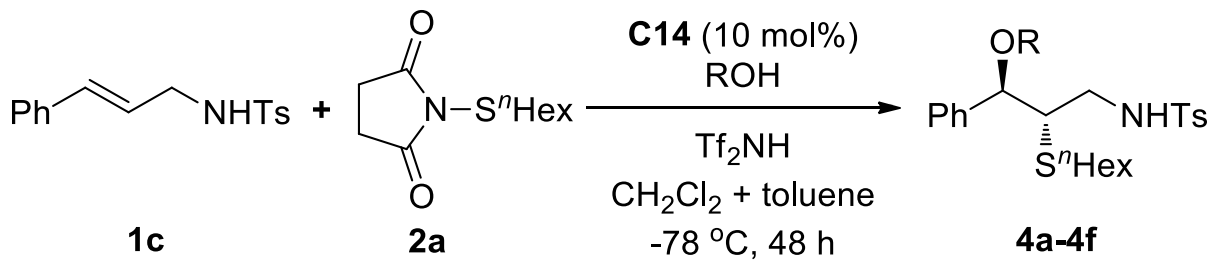


General procedure for the synthesis of $\mathbf{4 a}, \mathbf{4 e}$, and $\mathbf{4 f}$

To a 10-mL Schlenk flask charged with a magnetic stirring bar were added 1c (28.7 $\mathrm{mg}, 0.1 \mathrm{mmol})$, sulfur reagent $2 \mathbf{a}(32.3 \mathrm{mg}, 0.15 \mathrm{mmol})$, catalyst C14 (5.8 $\mathrm{mg}, 10$ mol\%) and $\mathrm{CH}_{2} \mathrm{Cl}_{2} /$ toluene $(2 \mathrm{~mL} / 2 \mathrm{~mL})$ subsequently. The flask was placed into a cooling bath at $-78{ }^{\circ} \mathrm{C}$. $\mathrm{Tf}_{2} \mathrm{NH}(70.3 \mathrm{mg}, 0.25 \mathrm{mmol})$ was added after the solution was stirred for about $5 \mathrm{~min}$ at $-78{ }^{\circ} \mathrm{C}$. After another $5 \mathrm{~min}$, a solution of $\mathrm{ROH}(0.2 \mathrm{mmol}, 2$ equiv) in DCM $(100 \mu \mathrm{L})$ was added. The resulting solution was stirred at $-78{ }^{\circ} \mathrm{C}$ for $48 \mathrm{~h}$, and then quenched with $5 \% \mathrm{NaHCO}_{3}$ aqueous solution $(4 \mathrm{~mL})$ and extracted with DCM $(5 \mathrm{~mL} \times 3)$. The organic layers were dried over $\mathrm{Na}_{2} \mathrm{SO}_{4}$, filtered, and concentrated under reduced pressure. The residue was purified by flash silica gel column chromatography to yield the corresponding product.

General procedure for the synthesis of $\mathbf{4 b} \mathbf{b}-\mathbf{4 d}$

To a 10-mL Schlenk flask charged with a magnetic stirring bar were added 1c (28.7 $\mathrm{mg}, 0.1 \mathrm{mmol})$, sulfur reagent $\mathbf{2 a}(32.3 \mathrm{mg}, 0.15 \mathrm{mmol})$, catalyst C14 (5.8 $\mathrm{mg}, 10$ mol\%) and $\mathrm{CH}_{2} \mathrm{Cl}_{2} /$ toluene $(2 \mathrm{~mL} / 2 \mathrm{~mL})$ subsequently. The flask was placed into a cooling bath at $-78{ }^{\circ} \mathrm{C}$. $\mathrm{Tf}_{2} \mathrm{NH}(70.3 \mathrm{mg}, 0.25 \mathrm{mmol})$ was added after the solution was stirred for about $5 \mathrm{~min}$ at $-78{ }^{\circ} \mathrm{C}$. After another $5 \mathrm{~min}$, ROH $(0.2 \mathrm{mmol}, 2$ equiv) was added. The resulting solution was stirred at $-78{ }^{\circ} \mathrm{C}$ for $48 \mathrm{~h}$, and then quenched with $5 \% \mathrm{NaHCO}_{3}$ aqueous solution $(4 \mathrm{~mL})$ and extracted with $\mathrm{DCM}(5 \mathrm{~mL} \times 3)$. The organic layers were dried over $\mathrm{Na}_{2} \mathrm{SO}_{4}$, filtered, and concentrated under reduced pressure. The residue was purified by flash silica gel column chromatography to yield the corresponding product.

\subsection{Characterization data of difunctionalization products}

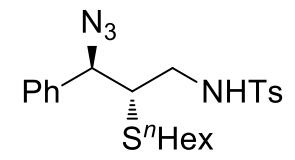

\section{$N$-((2S,3R)-3-Azido-2-(hexylthio)-3-phenylpropyl)-4-methylbenzenesulfonamide}

(3c): Flash column chromatography (eluent: PE/EtOAc $=15 / 1$ to $5 / 1, \mathrm{v} / \mathrm{v}$ ) to afford 3c as a colorless oil. $41.0 \mathrm{mg}, 92 \%$ yield, $94 \%$ ee with $>99: 1 d r .[\alpha]^{25} \mathrm{D}=-95.4(\mathrm{c}=$ 0.2, $\left.\mathrm{CHCl}_{3}\right) .{ }^{1} \mathrm{H}$ NMR $\left(400 \mathrm{MHz}, \mathrm{CDCl}_{3}\right) \delta 7.73(\mathrm{~d}, J=8.2 \mathrm{~Hz}, 2 \mathrm{H}), 7.40-7.33(\mathrm{~m}$, $3 \mathrm{H}), 7.32(\mathrm{~d}, J=8.2 \mathrm{~Hz}, 2 \mathrm{H}), 7.27-7.21(\mathrm{~m}, 2 \mathrm{H}), 5.13(\mathrm{dd}, J=7.3,5.3 \mathrm{~Hz}, 1 \mathrm{H}), 4.49$ $(\mathrm{d}, J=8.2 \mathrm{~Hz}, 1 \mathrm{H}), 3.42-3.30(\mathrm{~m}, 1 \mathrm{H}), 3.04-2.94(\mathrm{~m}, 1 \mathrm{H}), 2.79(\mathrm{td}, J=7.9,5.3 \mathrm{~Hz}$, $1 \mathrm{H}), 2.44(\mathrm{~s}, 3 \mathrm{H}), 2.20-2.01(\mathrm{~m}, 2 \mathrm{H}), 1.37-1.09(\mathrm{~m}, 8 \mathrm{H}), 0.86(\mathrm{t}, J=7.2 \mathrm{~Hz}, 3 \mathrm{H})$. ${ }^{13} \mathrm{C}$ NMR $\left(101 \mathrm{MHz}, \mathrm{CDCl}_{3}\right) \delta 143.59,136.90,136.82,129.79,128.84,128.77$, 127.65, 127.18, 68.47, 51.17, 43.75, 32.04, 31.22, 29.28, 28.31, 22.47, 21.55, 14.01. HR-ESI-MS $m / z$ calcd. for $\left.\mathrm{C}_{22} \mathrm{H}_{29} \mathrm{~N}_{4} \mathrm{O}_{2} \mathrm{~S}_{2}[\mathrm{M}-\mathrm{H}]\right]^{-}: 445.1752$, found: 445.1737. HPLC (Daicel Chiralpak AD-H column, $i-\mathrm{PrOH} /$ hexane $=5 / 95,1 \mathrm{~mL} / \mathrm{min}, 235 \mathrm{~nm}$ ) $\mathrm{t}_{1}=19.4$ $\min$ (minor), $\mathrm{t}_{2}=20.6 \min$ (major). 
$\mathrm{mAU}$

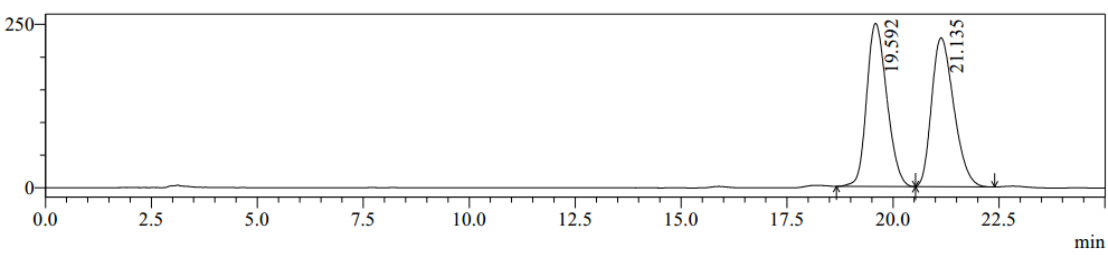

Peak Table

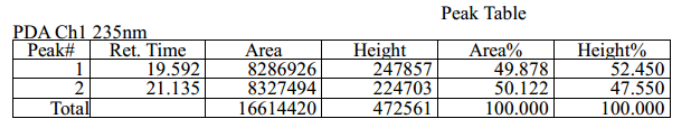

$\mathrm{mAU}$

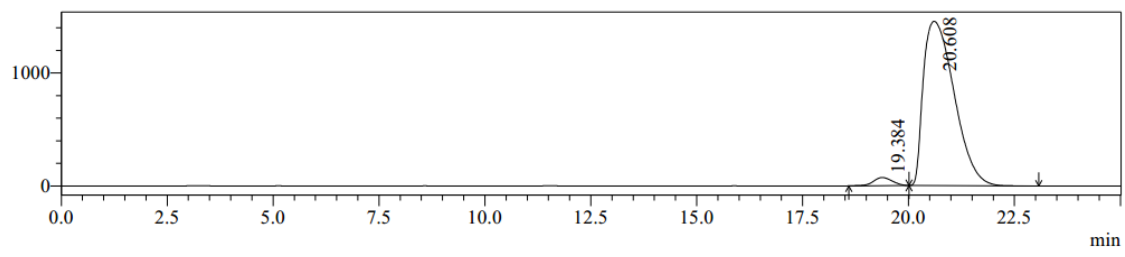

Peak Table

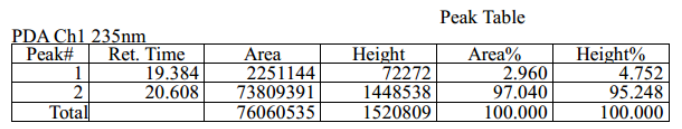

$\overbrace{\mathrm{NHTS}}^{\mathrm{N}_{\mathrm{S}}}$

$N$-((2S,3R)-3-Azido-3-phenyl-2-(propylthio)propyl)-4-methylbenzenesulfonamide

(3e): Flash column chromatography (eluent: PE/EtOAc $=15 / 1$ to $5 / 1, \mathrm{v} / \mathrm{v}$ ) to afford $3 \mathbf{e}$ a as colorless oil. $36.4 \mathrm{mg}, 90 \%$ yield, $95 \%$ ee with $31: 1 d r .[\alpha]^{25} \mathrm{D}=-112.1(\mathrm{c}=0.2$, $\left.\mathrm{CHCl}_{3}\right) .{ }^{1} \mathrm{H} \mathrm{NMR}\left(400 \mathrm{MHz}, \mathrm{CDCl}_{3}\right) \delta 7.73(\mathrm{~d}, J=8.2 \mathrm{~Hz}, 2 \mathrm{H}), 7.42-7.34(\mathrm{~m}, 3 \mathrm{H})$, $7.31(\mathrm{~d}, J=8.2 \mathrm{~Hz}, 2 \mathrm{H}), 7.28-7.22(\mathrm{~m}, 2 \mathrm{H}), 5.17(\mathrm{t}, J=5.5 \mathrm{~Hz}, 1 \mathrm{H}), 4.51(\mathrm{~d}, J=8.3$ $\mathrm{Hz}, 1 \mathrm{H}), 3.44-3.26(\mathrm{~m}, 1 \mathrm{H}), 2.99$ (ddd, $J=13.8,9.5,5.5 \mathrm{~Hz}, 1 \mathrm{H}), 2.80$ (td, $J=7.8$, $4.7 \mathrm{~Hz}, 1 \mathrm{H}), 2.43(\mathrm{~s}, 3 \mathrm{H}), 2.16-2.02(\mathrm{~m}, 2 \mathrm{H}), 1.42-1.28(\mathrm{~m}, 2 \mathrm{H}), 0.79(\mathrm{t}, J=7.3$ $\mathrm{Hz}, 3 \mathrm{H}) .{ }^{13} \mathrm{C}$ NMR $\left(101 \mathrm{MHz}, \mathrm{CDCl}_{3}\right) \delta 143.71,136.96,136.89,129.87,128.92$, $128.85,127.73,127.26,68.52,51.19,43.85,34.09,22.78,21.64,13.33$. HR-ESI-MS $m / z$ calcd. for $\mathrm{C}_{19} \mathrm{H}_{23} \mathrm{~N}_{4} \mathrm{O}_{2} \mathrm{~S}_{2}[\mathrm{M}-\mathrm{H}]$ ]: 403.1268, found: 403.1275. HPLC (Daicel Chiralpak OJ-H column, $i-\mathrm{PrOH} /$ hexane $=5 / 95,1 \mathrm{~mL} / \mathrm{min}, 206 \mathrm{~nm}) \mathrm{t}_{1}=27.0 \mathrm{~min}$ (major), $\mathrm{t}_{2}=32.6 \mathrm{~min}$ (minor).

$\mathrm{mAU}$
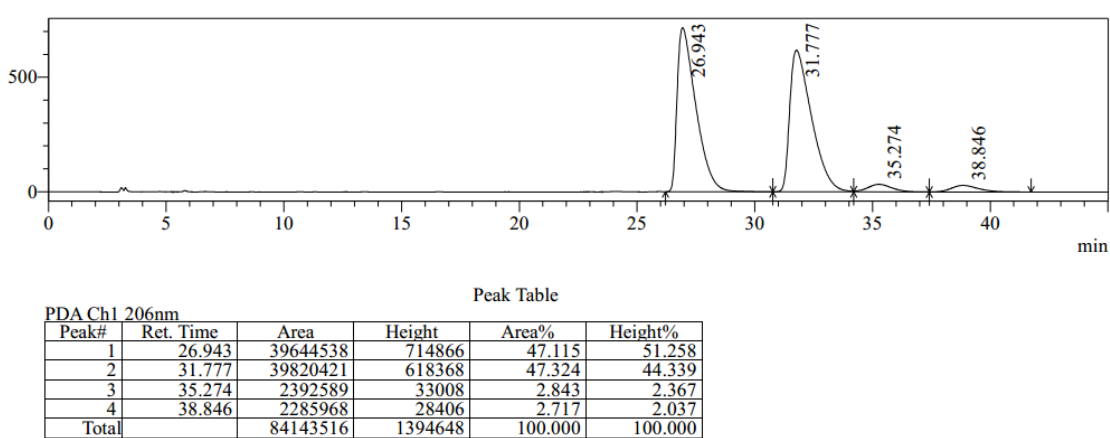

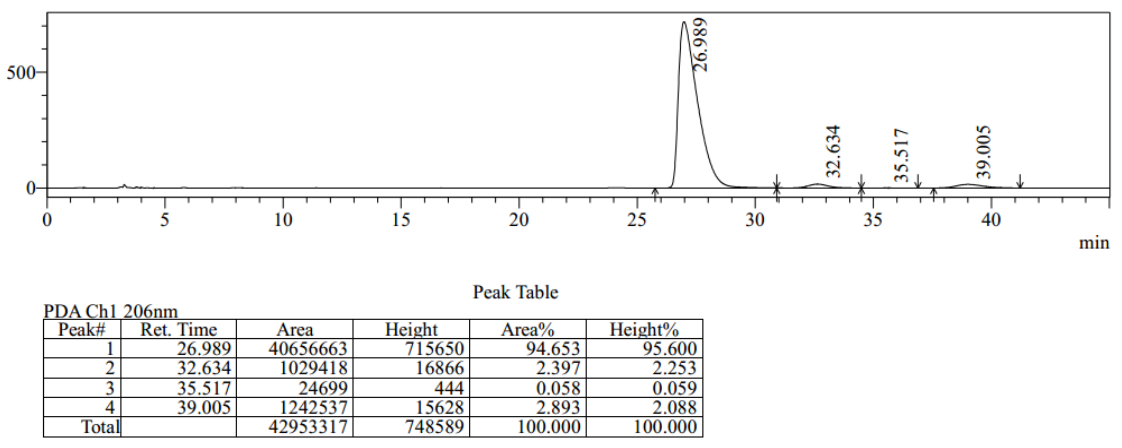

$\overbrace{M}^{\mathrm{NHT}}$

$N$-((2S,3R)-3-Azido-2-(isopentylthio)-3-phenylpropyl)-4-methylbenzenesulfonami de (3f): Flash column chromatography (eluent: PE/EtOAc $=15 / 1$ to 5/1, v/v) to afford 3f as a colorless oil. $37.2 \mathrm{mg}, 86 \%$ yield, 94\% ee with $>99: 1 d r$. $[\alpha]^{25}{ }_{\mathrm{D}}=-110.4(\mathrm{c}=$ 0.2, $\left.\mathrm{CHCl}_{3}\right) .{ }^{1} \mathrm{H} \mathrm{NMR}\left(400 \mathrm{MHz}, \mathrm{CDCl}_{3}\right) \delta 7.74(\mathrm{~d}, J=8.2 \mathrm{~Hz}, 2 \mathrm{H}), 7.41-7.33(\mathrm{~m}$, $4 \mathrm{H}), 7.31(\mathrm{~d}, J=8.2 \mathrm{~Hz}, 2 \mathrm{H}), 7.27-7.22(\mathrm{~m}, 2 \mathrm{H}), 5.23-5.10(\mathrm{~m}, 1 \mathrm{H}), 4.50(\mathrm{~d}, J=$ $8.2 \mathrm{~Hz}, 1 \mathrm{H}), 3.43-3.30(\mathrm{~m}, 1 \mathrm{H}), 3.04-2.92(\mathrm{~m}, 1 \mathrm{H}), 2.79(\mathrm{td}, J=8.2,4.7 \mathrm{~Hz}, 1 \mathrm{H})$, $2.43(\mathrm{~s}, 3 \mathrm{H}), 2.16-1.99(\mathrm{~m}, 2 \mathrm{H}), 1.42(\mathrm{tq}, J=13.2,6.7 \mathrm{~Hz}, 1 \mathrm{H}), 1.19(\mathrm{dd}, J=14.8$, $7.5 \mathrm{~Hz}, 2 \mathrm{H}), 0.76(\mathrm{t}, J=6.7 \mathrm{~Hz}, 6 \mathrm{H}) .{ }^{13} \mathrm{C} \mathrm{NMR}\left(101 \mathrm{MHz}, \mathrm{CDCl}_{3}\right) \delta 143.70,136.99$, 136.92, 129.89, 128.95, 128.87, 127.78, 127.27, 68.58, 51.28, 43.87, 38.32, 30.18, 27.38, 22.21, 22.17, 21.64. HR-ESI-MS $\mathrm{m} / z$ calcd. for $\mathrm{C}_{21} \mathrm{H}_{27} \mathrm{~N}_{4} \mathrm{O}_{2} \mathrm{~S}_{2}[\mathrm{M}-\mathrm{H}]^{-}$: 431.1581, found: 431.1591. HPLC (Daicel Chiralpak OD-H column, $i$-PrOH/hexane $=3 / 97,1 \mathrm{~mL} / \mathrm{min}, 223 \mathrm{~nm}$ ) $\mathrm{t}_{1}=20.2 \mathrm{~min}$ (minor), $\mathrm{t}_{2}=22.7 \mathrm{~min}$ (major).

$\mathrm{mAU}$
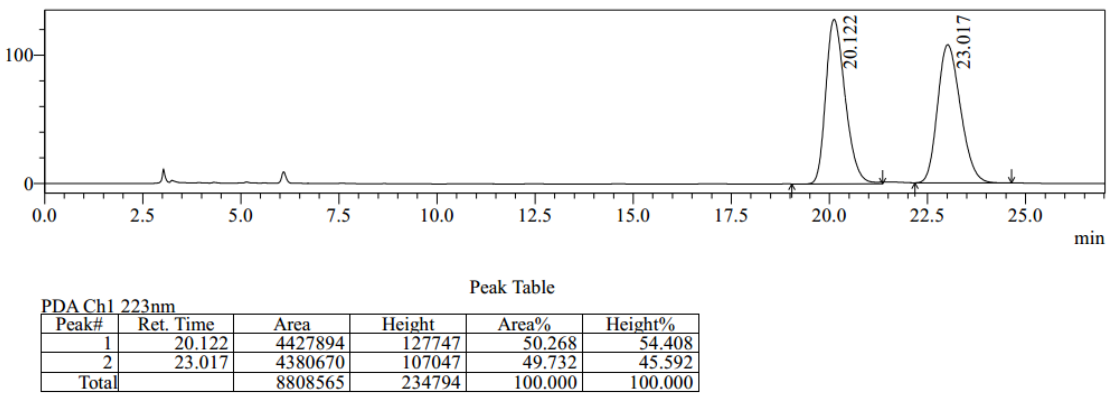
$\mathrm{mAU}$
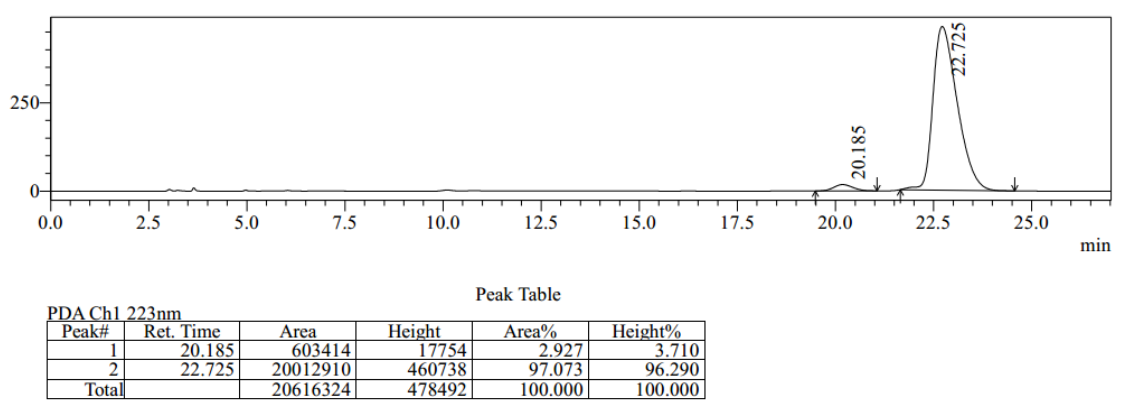

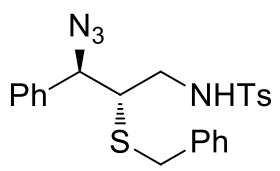

$N$-((2S,3R)-3-Azido-2-(benzylthio)-3-phenylpropyl)-4-methylbenzenesulfonamide (3g): Flash column chromatography (eluent: PE/EtOAc $=15 / 1$ to $5 / 1, \mathrm{v} / \mathrm{v}$ ) to afford 3g as a colorless oil. $39.3 \mathrm{mg}, 87 \%$ yield, $90 \%$ ee with $49: 1 d r .[\alpha]^{25} \mathrm{D}=-95.1(\mathrm{c}=0.2$, $\left.\mathrm{CHCl}_{3}\right) .{ }^{1} \mathrm{H} \mathrm{NMR}\left(400 \mathrm{MHz}, \mathrm{CDCl}_{3}\right) \delta 7.64(\mathrm{~d}, J=8.2 \mathrm{~Hz}, 2 \mathrm{H}), 7.37-7.31(\mathrm{~m}, 3 \mathrm{H})$, $7.26(\mathrm{~m}, 5 \mathrm{H}), 7.14-7.08(\mathrm{~m}, 4 \mathrm{H}), 5.08-4.97(\mathrm{~m}, 1 \mathrm{H}), 4.42(\mathrm{~d}, J=7.7 \mathrm{~Hz}, 1 \mathrm{H}), 3.42$ - $3.30(\mathrm{~m}, 2 \mathrm{H}), 3.20$ (ddd, $J=13.2,7.6,4.6 \mathrm{~Hz}, 1 \mathrm{H}), 3.07-2.94(\mathrm{~m}, 1 \mathrm{H}), 2.74$ (td, $J$ $=7.7,4.6 \mathrm{~Hz}, 1 \mathrm{H}), 2.42(\mathrm{~s}, 3 \mathrm{H}) .{ }^{13} \mathrm{C} \mathrm{NMR}\left(101 \mathrm{MHz}, \mathrm{CDCl}_{3}\right) \delta 143.63,137.21$, $136.84,136.76,129.85,129.00,128.85,128.83,128.80,127.62,127.58,127.23$, 68.30, 50.37, 43.42, 36.46, 21.64. HR-ESI-MS m/z calcd. for $\mathrm{C}_{23} \mathrm{H}_{23} \mathrm{~N}_{4} \mathrm{O}_{2} \mathrm{~S}_{2}[\mathrm{M}-\mathrm{H}]^{-}$: 451.1268, found: 451.1279. HPLC (Daicel Chiralpak AD-H column, $i-\mathrm{PrOH} /$ hexane $=10 / 90,1 \mathrm{~mL} / \min , 223 \mathrm{~nm}$ ) $\mathrm{t}_{1}=19.6 \min$ (major), $\mathrm{t}_{2}=21.3 \min$ (minor).

$\mathrm{mAU}$
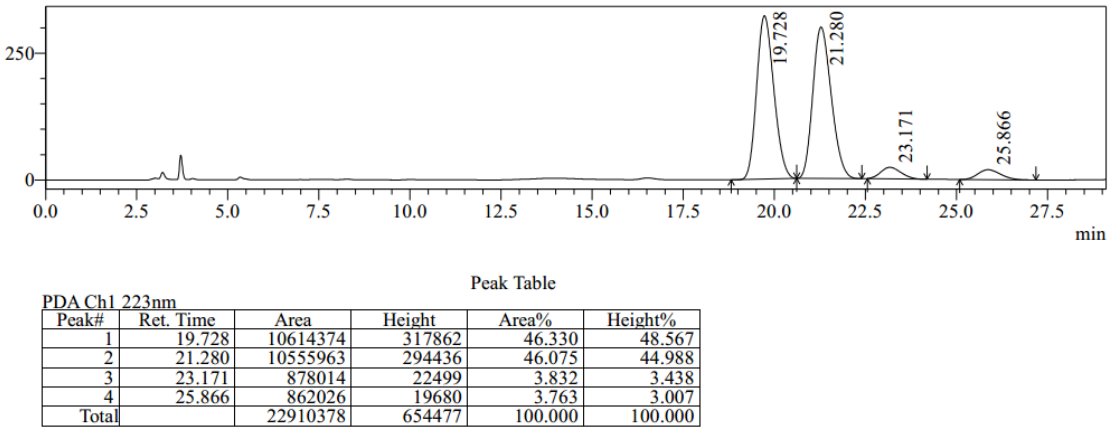

$\mathrm{mAU}$
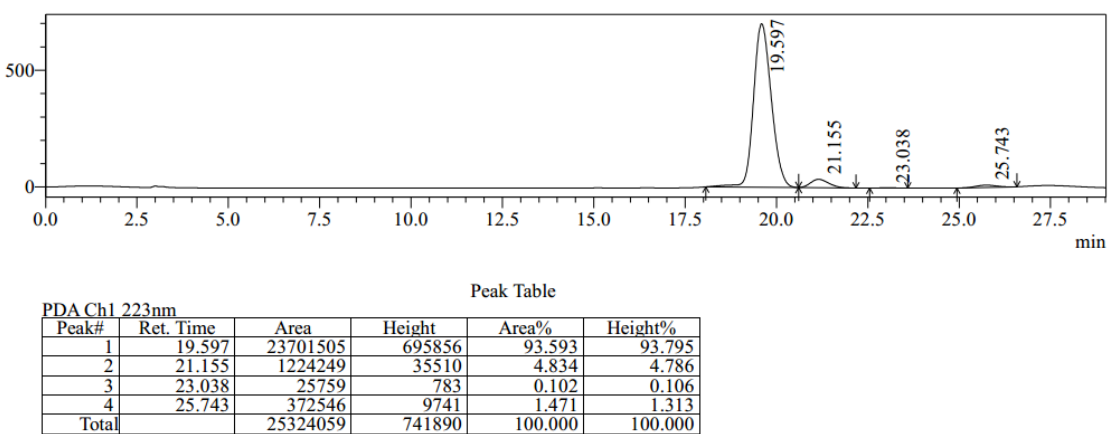
<smiles></smiles>

\section{$N$-((2S,3R)-3-Azido-2-(phenethylthio)-3-phenylpropyl)-4-methylbenzenesulfona} mide (3h): Flash column chromatography (eluent: PE/EtOAc $=15 / 1$ to $5 / 1, \mathrm{v} / \mathrm{v}$ ) to afford $3 \mathbf{h}$ as a colorless oil. $42.9 \mathrm{mg}, 92 \%$ yield, $96 \%$ ee with $>99: 1 d r .[\alpha]^{25} \mathrm{D}=$ -107.4 (c = 0.2, $\left.\mathrm{CHCl}_{3}\right) .{ }^{1} \mathrm{H} \mathrm{NMR}\left(400 \mathrm{MHz}, \mathrm{CDCl}_{3}\right) \delta 7.70(\mathrm{~d}, J=8.1 \mathrm{~Hz}, 2 \mathrm{H}), 7.42$ $-7.31(\mathrm{~m}, 3 \mathrm{H}), 7.31-7.15(\mathrm{~m}, 7 \mathrm{H}), 7.00(\mathrm{~d}, J=7.1 \mathrm{~Hz}, 2 \mathrm{H}), 5.17(\mathrm{~s}, 1 \mathrm{H}), 4.48(\mathrm{~d}, J$ $=7.9 \mathrm{~Hz}, 1 \mathrm{H}), 3.41-3.24(\mathrm{~m}, 1 \mathrm{H}), 3.06-2.88(\mathrm{~m}, 1 \mathrm{H}), 2.81(\mathrm{td}, J=7.9,4.7 \mathrm{~Hz}, 1 \mathrm{H})$, $2.64-2.54(\mathrm{~m}, 2 \mathrm{H}), 2.44-2.29(\mathrm{~m}, 5 \mathrm{H}) .{ }^{13} \mathrm{C} \mathrm{NMR}\left(101 \mathrm{MHz}, \mathrm{CDCl}_{3}\right) \delta$ 143.66, $139.72,136.84,136.81,129.83,128.90,128.84,128.54,128.45,127.72,127.18$, 126.56, 68.39, 51.47, 43.86, 35.87, 33.45, 21.56. HR-ESI-MS $\mathrm{m} / \mathrm{z}$ calcd. for $\mathrm{C}_{24} \mathrm{H}_{25} \mathrm{~N}_{4} \mathrm{O}_{2} \mathrm{~S}_{2}[\mathrm{M}-\mathrm{H}]^{-}:$465.1424, found: 465.1430. HPLC (Daicel Chiralpak OD-H column, $i-\mathrm{PrOH} / \mathrm{h}$ exane $=10 / 90,1 \mathrm{~mL} / \mathrm{min}, 223 \mathrm{~nm}$ ) $\mathrm{t}_{1}=17.6 \mathrm{~min}$ (minor), $\mathrm{t}_{2}=23.0$ $\min$ (major).

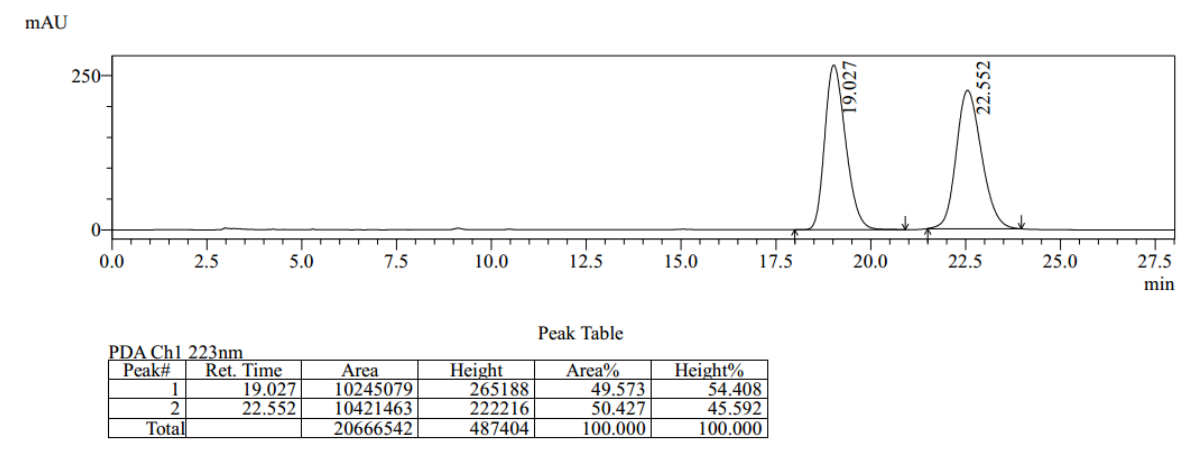

mAU
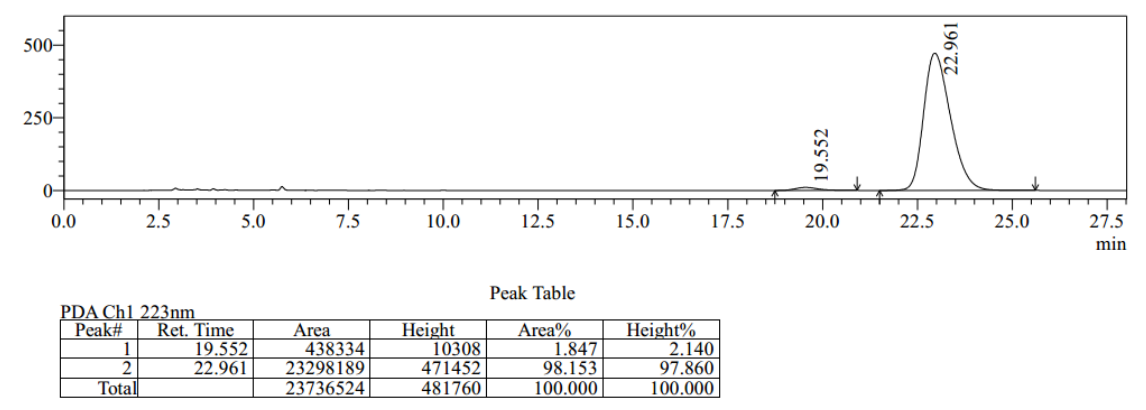

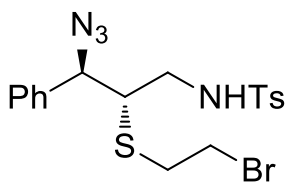

$N$-((2S,3R)-3-Azido-2-((2-bromoethyl)thio)-3-phenylpropyl)-4-methylbenzenesulf onamide (3i): Flash column chromatography (eluent: PE/EtOAc $=15 / 1$ to $5 / 1, \mathrm{v} / \mathrm{v}$ ) to afford 3i as a colorless oil. $38.4 \mathrm{mg}, 82 \%$ yield, $94 \%$ ee with $>99: 1 d r .[\alpha]^{25} \mathrm{D}=-151.0$ $\left(\mathrm{c}=0.2, \mathrm{CHCl}_{3}\right) .{ }^{1} \mathrm{H} \mathrm{NMR}\left(400 \mathrm{MHz}, \mathrm{CDCl}_{3}\right) \delta 7.76-7.71(\mathrm{~m}, 2 \mathrm{H}), 7.42-7.35(\mathrm{~m}$, $3 \mathrm{H}), 7.33(\mathrm{~d}, J=8.0 \mathrm{~Hz}, 2 \mathrm{H}), 7.29-7.23(\mathrm{~m}, 2 \mathrm{H}), 5.38-5.21(\mathrm{~m}, 1 \mathrm{H}), 4.56(\mathrm{~d}, J=$ 
8.0 Hz, 1H), $3.38(\mathrm{ddd}, J=13.7,7.8,4.8 \mathrm{~Hz}, 1 \mathrm{H}), 3.20-3.06(\mathrm{~m}, 2 \mathrm{H}), 3.05-2.95(\mathrm{~m}$, $1 \mathrm{H}), 2.86(\mathrm{td}, J=8.0,4.8 \mathrm{~Hz}, 1 \mathrm{H}), 2.64-2.47(\mathrm{~m}, 2 \mathrm{H}), 2.44(\mathrm{~s}, 3 \mathrm{H}) .{ }^{13} \mathrm{C}$ NMR $(101$ $\left.\mathrm{MHz}, \mathrm{CDCl}_{3}\right) \delta 143.90,136.78,136.49,129.96,129.13,128.96,127.78,127.20$, 68.30, 52.14, 44.37, 34.34, 30.05, 21.64. HR-ESI-MS $\mathrm{m} / \mathrm{z}$ calcd. for $\mathrm{C}_{18} \mathrm{H}_{21} \mathrm{BrN}_{4} \mathrm{NaO}_{2} \mathrm{~S}_{2}[\mathrm{M}+\mathrm{Na}]^{+}:$491.0182, found: 491.0182. HPLC (Daicel Chiralpak IC column, $i$-PrOH/hexane $=10 / 90,1 \mathrm{~mL} / \mathrm{min}, 235 \mathrm{~nm}) \mathrm{t}_{1}=21.8 \mathrm{~min}($ minor $), \mathrm{t}_{2}=$ 24.7 min (major).

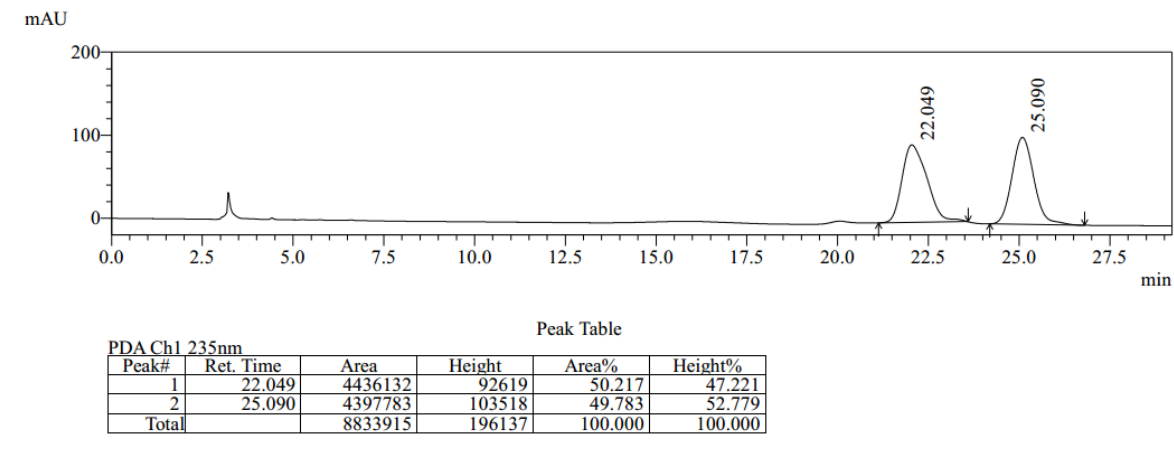

$\mathrm{mAU}$
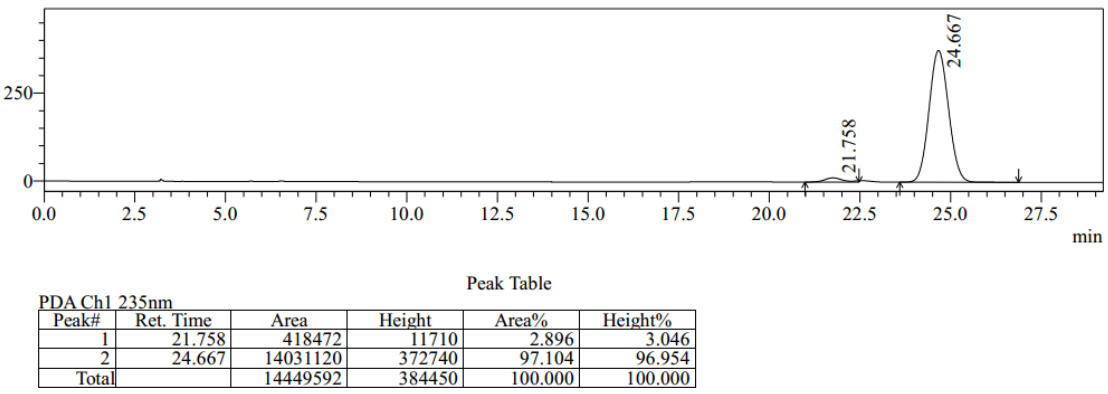<smiles>[13CH3]NC[C@H](SCCCCl)C(N)c1ccccc1</smiles>

$N$-((2S,3R)-3-Azido-2-((3-chloropropyl)thio)-3-phenylpropyl)-4-methylbenzenesu

Ifonamide (3j): Flash column chromatography (eluent: PE/EtOAc $=15 / 1$ to $3 / 1$, v/v) to afford $3 \mathbf{j}$ as a colorless oil. $36.4 \mathrm{mg}, 83 \%$ yield, $94 \%$ ee with $>99: 1 d r$. $[\alpha]^{25} \mathrm{D}=$ -43.5 (c = 0.2, $\left.\mathrm{CHCl}_{3}\right) .{ }^{1} \mathrm{H}$ NMR $\left(400 \mathrm{MHz}, \mathrm{CDCl}_{3}\right) \delta 7.73(\mathrm{~d}, J=8.2 \mathrm{~Hz}, 2 \mathrm{H}), 7.41-$ $7.35(\mathrm{~m}, 3 \mathrm{H}), 7.32(\mathrm{~d}, J=8.2 \mathrm{~Hz}, 2 \mathrm{H}), 7.29-7.24(\mathrm{~m}, 2 \mathrm{H}), 5.13(\mathrm{dd}, J=6.9,5.9 \mathrm{~Hz}$, $1 \mathrm{H}), 4.51(\mathrm{~d}, J=8.0 \mathrm{~Hz}, 1 \mathrm{H}), 3.47-3.28(\mathrm{~m}, 3 \mathrm{H}), 3.07-2.97(\mathrm{~m}, 1 \mathrm{H}), 2.83(\mathrm{td}, J=$ 8.0, $4.8 \mathrm{~Hz}, 1 \mathrm{H}), 2.44(\mathrm{~s}, 3 \mathrm{H}), 2.38-2.25(\mathrm{~m}, 2 \mathrm{H}), 1.83-1.68(\mathrm{~m}, 2 \mathrm{H}) .{ }^{13} \mathrm{C} \mathrm{NMR}$ $\left(101 \mathrm{MHz}, \mathrm{CDCl}_{3}\right) \delta 143.71,136.79,136.75,129.83,128.97,128.86,127.67,127.16$, 68.29, 51.55, 44.04, 42.99, 31.74, 29.02, 21.55. HR-ESI-MS $\mathrm{m} / \mathrm{z}$ calcd. for $\mathrm{C}_{19} \mathrm{H}_{22} \mathrm{~N}_{4} \mathrm{O}_{2} \mathrm{~S}_{2} \mathrm{Cl}[\mathrm{M}-\mathrm{H}]=:$ 437.0878, found: 437.0887. HPLC (Daicel Chiralpak OJ-H column, $i-\mathrm{PrOH} / \mathrm{hexane}=20 / 80,1 \mathrm{~mL} / \mathrm{min}, 223 \mathrm{~nm}$ ) $\mathrm{t}_{1}=14.9 \mathrm{~min}$ (major), $\mathrm{t}_{2}=17.5$ $\min$ (minor). 
$\mathrm{mAU}$
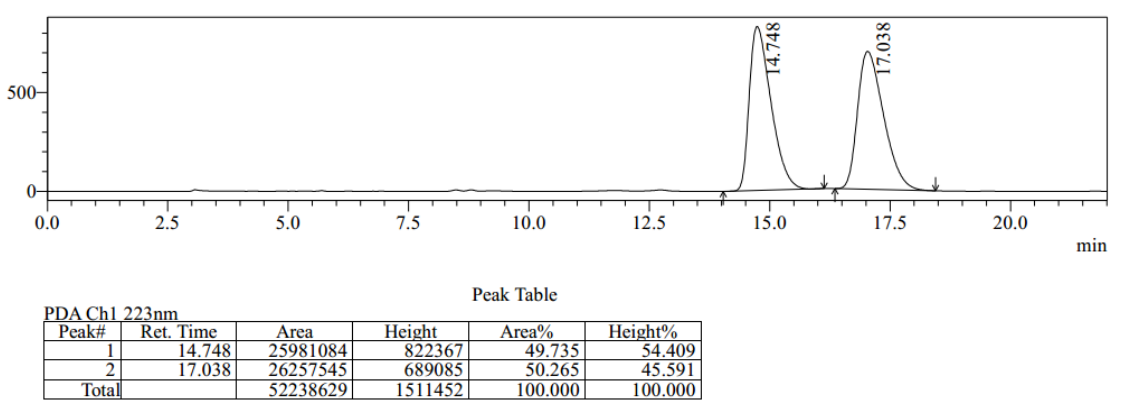

$\mathrm{mAU}$
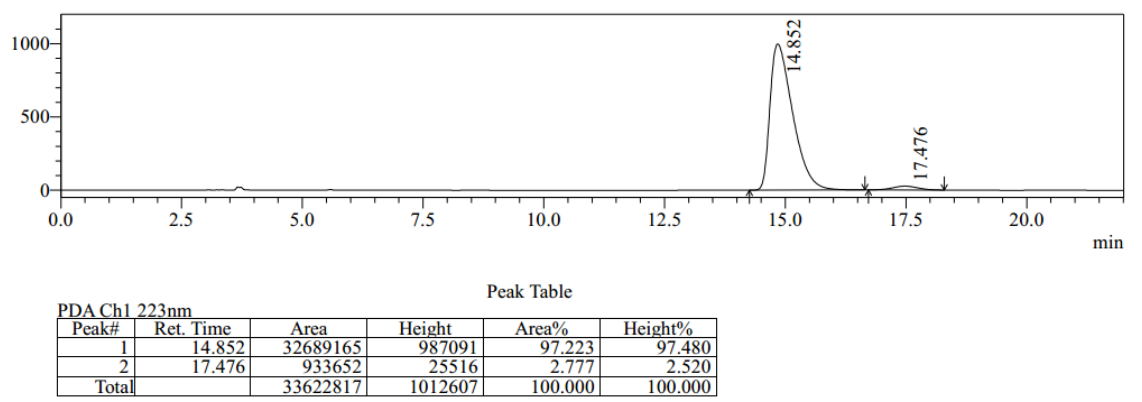

N

$N$-((2S,3R)-2-(Allylthio)-3-azido-3-phenylpropyl)-4-methylbenzenesulfonamide

(3k): Flash column chromatography (eluent: $\mathrm{PE} / \mathrm{EtOAc}=15 / 1$ to $5 / 1, \mathrm{v} / \mathrm{v}$ ) to afford 3k as a colorless oil. $25.3 \mathrm{mg}, 63 \%$ yield, $92 \%$ ee with $>99: 1 d r .[\alpha]^{25} \mathrm{D}=9.1(\mathrm{c}=0.2$, $\left.\mathrm{CHCl}_{3}\right) .{ }^{1} \mathrm{H} \mathrm{NMR}\left(400 \mathrm{MHz}, \mathrm{CDCl}_{3}\right) \delta 7.71(\mathrm{~d}, J=8.2 \mathrm{~Hz}, 2 \mathrm{H}), 7.40-7.34(\mathrm{~m}, 3 \mathrm{H})$, $7.30(\mathrm{~d}, J=8.2 \mathrm{~Hz}, 2 \mathrm{H}), 7.27-7.22(\mathrm{~m}, 2 \mathrm{H}), 5.55(\mathrm{ddt}, J=17.1,9.9,7.2 \mathrm{~Hz}, 1 \mathrm{H})$, $5.12(\mathrm{dt}, J=11.6,5.8 \mathrm{~Hz}, 1 \mathrm{H}), 5.07-4.88(\mathrm{~m}, 2 \mathrm{H}), 4.57(\mathrm{~d}, J=7.9 \mathrm{~Hz}, 1 \mathrm{H}), 3.34-$ $3.24(\mathrm{~m}, 1 \mathrm{H}), 3.08-2.98(\mathrm{~m}, 1 \mathrm{H}), 2.88-2.80(\mathrm{~m}, 2 \mathrm{H}), 2.69(\mathrm{dd}, J=13.7,7.3 \mathrm{~Hz}$, $1 \mathrm{H}), 2.43(\mathrm{~s}, 3 \mathrm{H}) .{ }^{13} \mathrm{C} \mathrm{NMR}\left(101 \mathrm{MHz}, \mathrm{CDCl}_{3}\right) \delta 143.66,136.84,133.66,129.85$, 129.04, 128.91, 128.84, 127.72, 127.23, 118.32, 68.35, 49.60, 43.55, 35.00, 21.62. R-ESI-MS $m / z$ calcd. for $\mathrm{C}_{19} \mathrm{H}_{21} \mathrm{~N}_{4} \mathrm{O}_{2} \mathrm{~S}_{2}[\mathrm{M}-\mathrm{H}]=$ : 401.1111, found: 401.1116. HPLC (Daicel Chiralpak OD-H column, $i-\mathrm{PrOH} /$ hexane $=5 / 95,1 \mathrm{~mL} / \mathrm{min}, 216 \mathrm{~nm}$ ) $\mathrm{t}_{1}=32.6$ $\min$ (minor), $\mathrm{t}_{2}=36.7$ min (major).

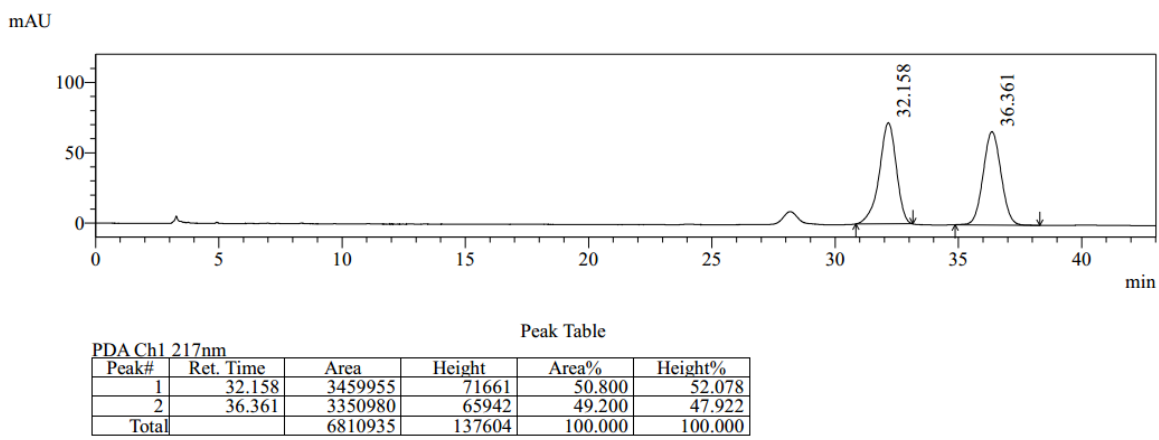



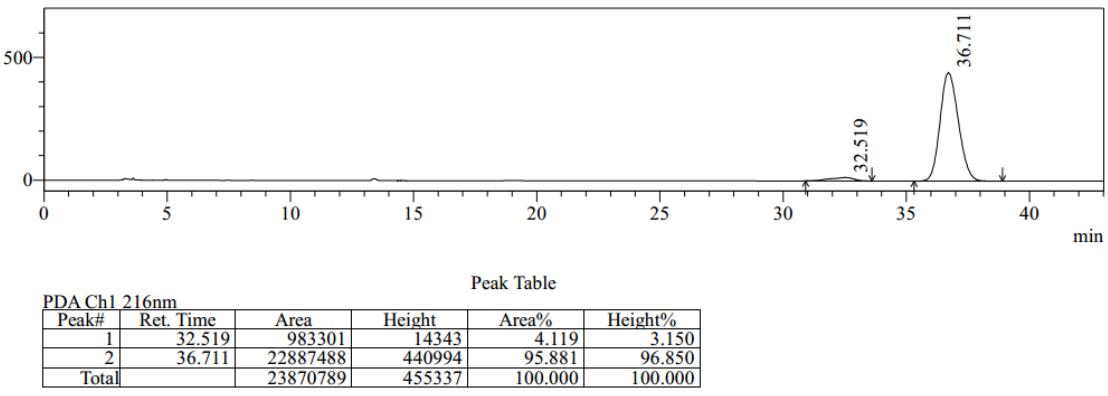

$\overbrace{\mathrm{CN}}^{\mathrm{NHTS}}$

$N$-((2S,3R)-3-Azido-2-((4-cyanobutyl)thio)-3-phenylpropyl)-4-methylbenzenesulf onamide (3I): Flash column chromatography (eluent: PE/EtOAc $=10 / 1$ to 3/1, v/v) to afford 31 as a colorless oil. $35.5 \mathrm{mg}, 80 \%$ yield, $94 \%$ ee with $>99: 1 d r .[\alpha]^{25}{ }_{\mathrm{D}}=-99.6$ $\left(\mathrm{c}=0.2, \mathrm{CHCl}_{3}\right) .{ }^{1} \mathrm{H} \mathrm{NMR}\left(400 \mathrm{MHz}, \mathrm{CDCl}_{3}\right) \delta 7.73(\mathrm{~d}, J=8.2 \mathrm{~Hz}, 2 \mathrm{H}), 7.41-7.35$ $(\mathrm{m}, 3 \mathrm{H}), 7.33(\mathrm{~d}, J=8.2 \mathrm{~Hz}, 2 \mathrm{H}), 7.29-7.26(\mathrm{~m}, 2 \mathrm{H}), 5.27(\mathrm{dd}, J=7.2,5.7 \mathrm{~Hz}, 1 \mathrm{H})$, $4.51(\mathrm{~d}, J=8.0 \mathrm{~Hz}, 1 \mathrm{H}), 3.35(\mathrm{ddd}, J=13.4,7.4,4.8 \mathrm{~Hz}, 1 \mathrm{H}), 3.09-2.94(\mathrm{~m}, 1 \mathrm{H})$, $2.84(\mathrm{td}, J=8.0,4.8 \mathrm{~Hz}, 1 \mathrm{H}), 2.44(\mathrm{~s}, 3 \mathrm{H}), 2.28-2.09(\mathrm{~m}, 4 \mathrm{H}), 1.58-1.38(\mathrm{~m}, 4 \mathrm{H})$. ${ }^{13} \mathrm{C}$ NMR $\left(101 \mathrm{MHz}, \mathrm{CDCl}_{3}\right) \delta 143.78,136.85,136.76,129.88,128.97,128.85$, 127.82 , 127.17, 119.39, 68.29, 51.49, 44.25, 31.29, 27.96, 24.05, 21.60, 16.65. HR-ESI-MS $m / z$ calcd. for $\mathrm{C}_{21} \mathrm{H}_{24} \mathrm{~N}_{5} \mathrm{O}_{2} \mathrm{~S}_{2}[\mathrm{M}-\mathrm{H}]^{-}:$442.1377, found: 442.1390. HPLC (Daicel Chiralpak OD-H column, $i$-PrOH/hexane $=12 / 88,1 \mathrm{~mL} / \mathrm{min}, 223 \mathrm{~nm}$ ) $\mathrm{t}_{1}=$ $34.0 \mathrm{~min}$ (major), $\mathrm{t}_{2}=37.2 \mathrm{~min}$ (minor).

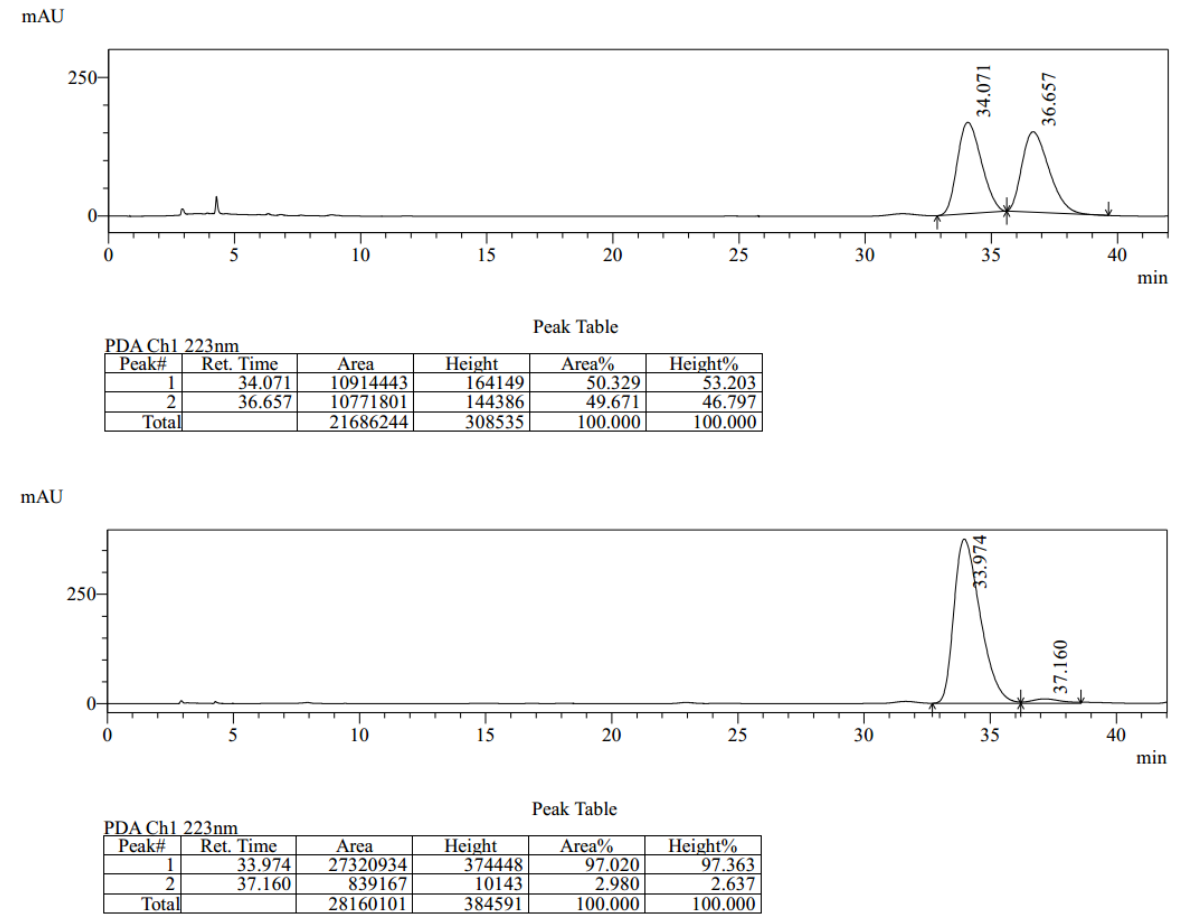


<smiles>NC(c1ccccc1)[C@H](CNC(N)(F)F)Sc1ccccc1</smiles>

$N$-((2S,3R)-3-Azido-3-phenyl-2-(phenylthio)propyl)-4-methylbenzenesulfonamide (3m): Flash column chromatography (eluent: PE/EtOAc $=15 / 1$ to $5 / 1, \mathrm{v} / \mathrm{v}$ ) to afford 3m as a white solid. $41.2 \mathrm{mg}, 94 \%$ yield, $96 \%$ ee with $>99: 1 d r$ mp $92.6-94.8^{\circ} \mathrm{C}$. $[\alpha]^{25} \mathrm{D}=-119.5\left(\mathrm{c}=0.2, \mathrm{CHCl}_{3}\right) .{ }^{1} \mathrm{H} \mathrm{NMR}\left(400 \mathrm{MHz}, \mathrm{CDCl}_{3}\right) \delta 7.64(\mathrm{~d}, J=8.3 \mathrm{~Hz}$, $2 \mathrm{H}), 7.36-7.29(\mathrm{~m}, 2 \mathrm{H}), 7.28-7.22(\mathrm{~m}, 3 \mathrm{H}), 7.22-7.20(\mathrm{~m}, 1 \mathrm{H}), 7.20-7.12(\mathrm{~m}$, $5 \mathrm{H}), 5.26(\mathrm{t}, J=6.1 \mathrm{~Hz}, 1 \mathrm{H}), 4.59(\mathrm{~d}, J=7.1 \mathrm{~Hz}, 1 \mathrm{H}), 3.37-3.18(\mathrm{~m}, 2 \mathrm{H}), 3.20-$ $2.97(\mathrm{~m}, 1 \mathrm{H}), 2.42(\mathrm{~s}, 3 \mathrm{H}) .{ }^{13} \mathrm{C} \mathrm{NMR}\left(101 \mathrm{MHz}, \mathrm{CDCl}_{3}\right) \delta 143.59,136.68,136.40$, 133.05, 132.46, 129.80, 129.23, 128.83, 128.12, 127.46, 127.17, 67.24, 55.00, 43.11, 21.58. HR-ESI-MS $m / z$ calcd. for $\mathrm{C}_{22} \mathrm{H}_{21} \mathrm{~N}_{4} \mathrm{O}_{2} \mathrm{~S}_{2}[\mathrm{M}-\mathrm{H}]^{-}: 437.1111$, found: 437.1114 . HPLC (Daicel Chiralpak OD-H column, $i$-PrOH/hexane = 10/90, $1 \mathrm{~mL} / \mathrm{min}, 223 \mathrm{~nm}$ ) $\mathrm{t}_{1}=14.9 \min$ (major), $\mathrm{t}_{2}=17.8 \mathrm{~min}$ (minor).
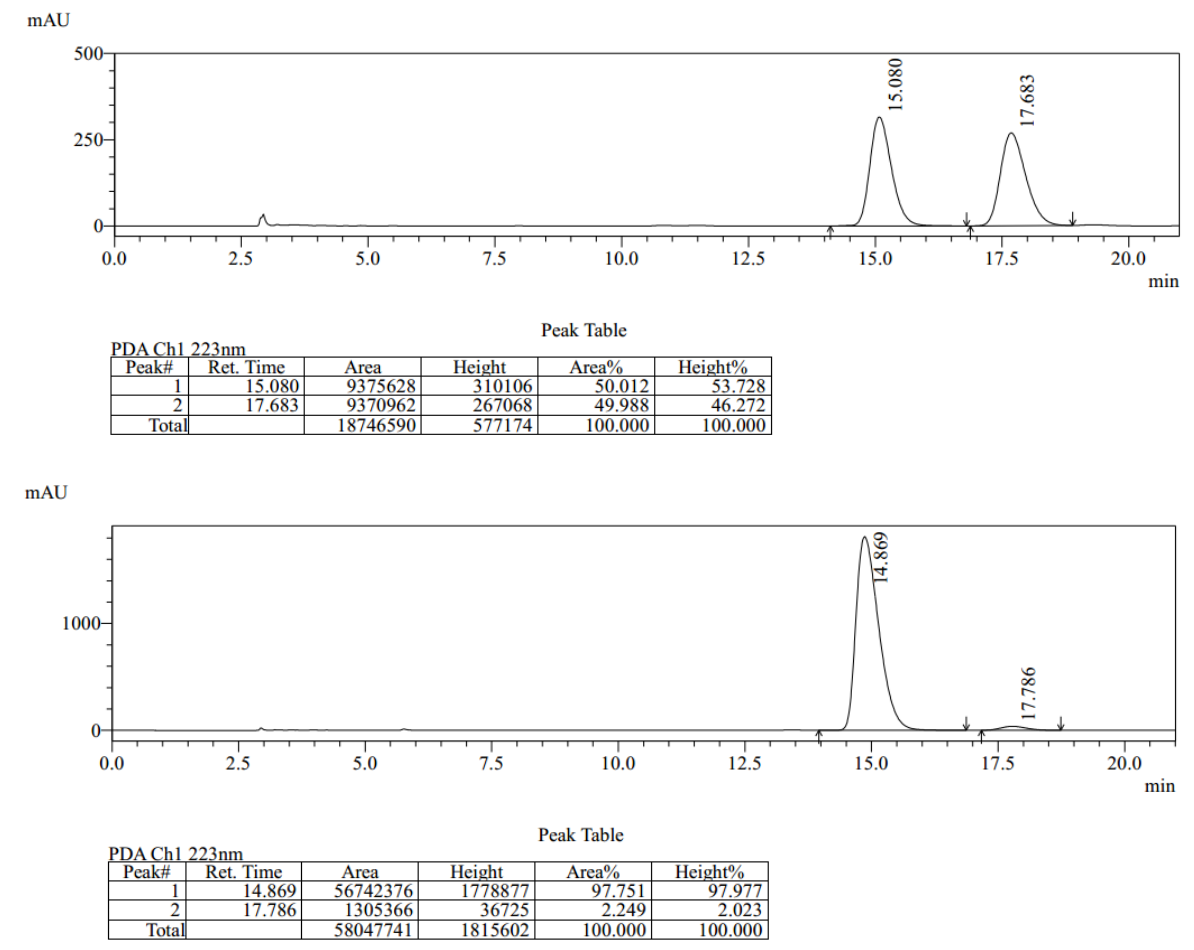<smiles>CNC[C@@H](Sc1ccccc1C)C(N)c1ccccc1</smiles>

$N$-((2S,3R)-3-Azido-3-phenyl-2-(o-tolylthio)propyl)-4-methylbenzenesulfonamide (3n): Flash column chromatography (eluent: PE/EtOAc $=15 / 1$ to $5 / 1, \mathrm{v} / \mathrm{v}$ ) to afford 3n as a colorless oil. $42.0 \mathrm{mg}, 93 \%$ yield, $97 \%$ ee with $>99: 1 d r .[\alpha]^{25} \mathrm{D}=-78.2(\mathrm{c}=$ 0.2, $\left.\mathrm{CHCl}_{3}\right) .{ }^{1} \mathrm{H} \mathrm{NMR}\left(400 \mathrm{MHz}, \mathrm{CDCl}_{3}\right) \delta 7.59(\mathrm{~d}, J=8.1 \mathrm{~Hz}, 2 \mathrm{H}), 7.36-7.28(\mathrm{~m}$, 
$3 \mathrm{H}), 7.24(\mathrm{~d}, J=7.9 \mathrm{~Hz}, 2 \mathrm{H}), 7.19-7.11(\mathrm{~m}, 5 \mathrm{H}), 7.09-7.01(\mathrm{~m}, 1 \mathrm{H}), 5.14(\mathrm{t}, J=$ $6.0 \mathrm{~Hz}, 1 \mathrm{H}), 4.62(\mathrm{~d}, J=6.9 \mathrm{~Hz}, 1 \mathrm{H}), 3.28(\mathrm{dd}, J=11.7,5.9 \mathrm{~Hz}, 1 \mathrm{H}), 3.24-3.08(\mathrm{~m}$, 2H), 2.42 (s, 3H), $2.20(\mathrm{~s}, 3 \mathrm{H}) .{ }^{13} \mathrm{C}$ NMR $\left(101 \mathrm{MHz}, \mathrm{CDCl}_{3}\right) \delta 143.60,140.69,136.75$, $136.62,133.26,132.03,130.81,129.83,128.90,128.25,127.40,127.21,126.79$, 67.42, 53.97, 43.23, 21.63, 20.73. HR-ESI-MS $\mathrm{m} / \mathrm{z}$ calcd. for $\mathrm{C}_{23} \mathrm{H}_{24} \mathrm{~N}_{4} \mathrm{NaO}_{2} \mathrm{~S}_{2}$ $[\mathrm{M}+\mathrm{Na}]^{+}:$475.1233, found: 475.1235. HPLC (Daicel Chiralpak IA column, $i$-PrOH/hexane $=10 / 90,1 \mathrm{~mL} / \mathrm{min}, 215 \mathrm{~nm}$ ) $\mathrm{t}_{1}=13.8 \mathrm{~min}$ (major), $\mathrm{t}_{2}=15.1 \mathrm{~min}$ (minor).
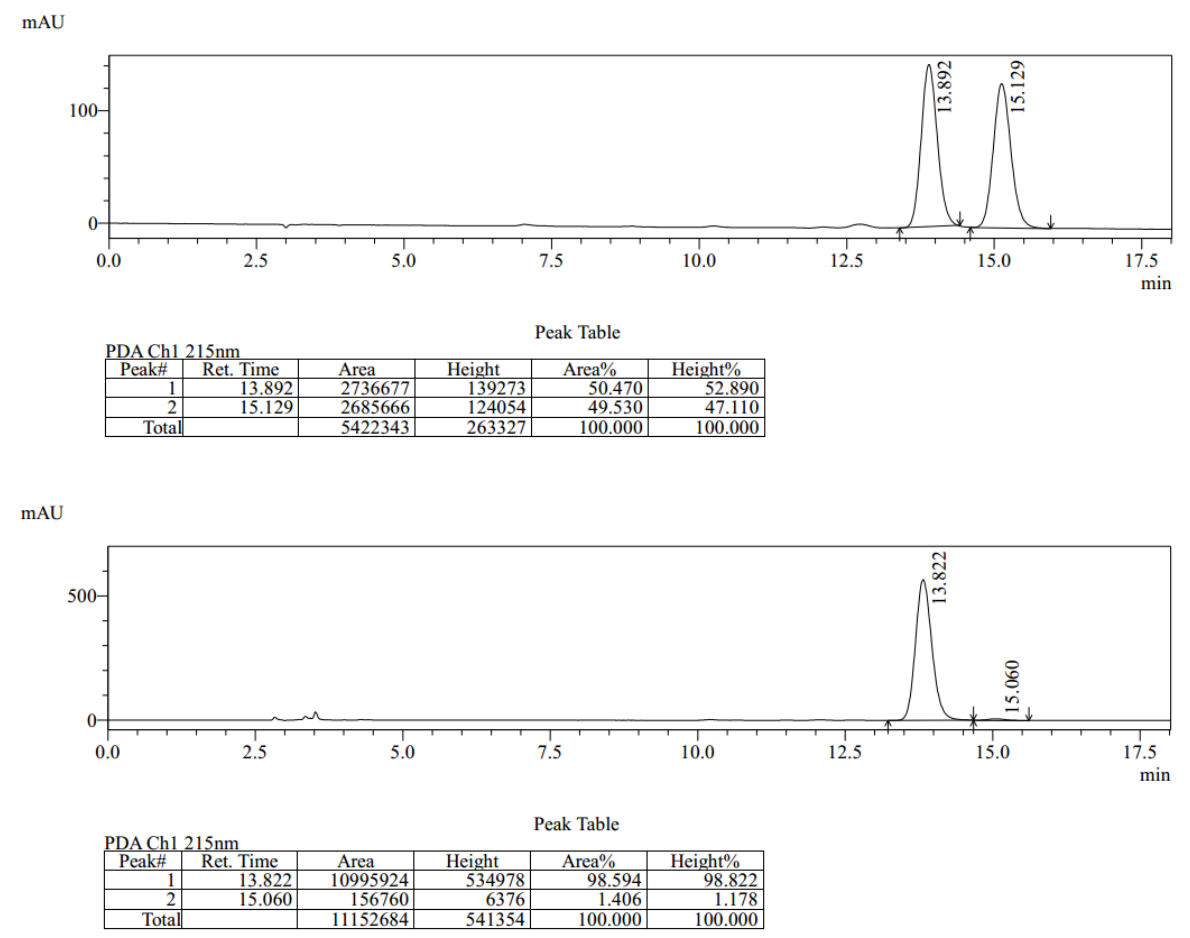<smiles>Cc1ccc(SC(CN[135I])C([NH3+])c2ccccc2)cc1</smiles>

$N$-((2S,3R)-3-Azido-3-phenyl-2-(p-tolylthio)propyl)-4-methylbenzenesulfonamide

(3o): Flash column chromatography (eluent: $\mathrm{PE} / \mathrm{EtOAc}=15 / 1$ to $5 / 1, \mathrm{v} / \mathrm{v}$ ) to afford 3o as a white solid. $42.0 \mathrm{mg}, 93 \%$ yield, $96 \%$ ee with $>99: 1 d r . \mathrm{mp} 82.9-84.2{ }^{\circ} \mathrm{C}$. $[\alpha]^{25} \mathrm{D}=-53.7\left(\mathrm{c}=0.2, \mathrm{CHCl}_{3}\right) .{ }^{1} \mathrm{H} \mathrm{NMR}\left(400 \mathrm{MHz}, \mathrm{CDCl}_{3}\right) \delta 7.65(\mathrm{~d}, J=8.2 \mathrm{~Hz}$, $2 \mathrm{H}), 7.37-7.30(\mathrm{~m}, 3 \mathrm{H}), 7.30-7.24(\mathrm{~m}, 2 \mathrm{H}), 7.15(\mathrm{~d}, J=6.6 \mathrm{~Hz}, 2 \mathrm{H}), 7.07-6.97$ $(\mathrm{m}, 4 \mathrm{H}), 5.19-5.12(\mathrm{~m}, 1 \mathrm{H}), 4.54(\mathrm{~d}, J=7.4 \mathrm{~Hz}, 1 \mathrm{H}), 3.28$ (ddd, $J=13.1,7.2,4.3$ $\mathrm{Hz}, 1 \mathrm{H}), 3.22-3.16(\mathrm{~m}, 1 \mathrm{H}), 3.14-3.06(\mathrm{~m}, 1 \mathrm{H}), 2.44(\mathrm{~s}, 3 \mathrm{H}), 2.31(\mathrm{~s}, 3 \mathrm{H}) .{ }^{13} \mathrm{C}$ NMR $\left(101 \mathrm{MHz}, \mathrm{CDCl}_{3}\right) \delta 143.63,138.66,136.79,136.61,133.71,130.12,129.86$, 128.93, 128.47, 127.52, 127.28, 67.25, 55.17, 42.99, 21.67, 21.26. HR-ESI-MS m/z calcd. for $\mathrm{C}_{23} \mathrm{H}_{23} \mathrm{~N}_{4} \mathrm{O}_{2} \mathrm{~S}_{2}[\mathrm{M}-\mathrm{H}]=4$ 451.1268, found: 451.1276. HPLC (Daicel Chiralpak OD-H column, $i$-PrOH/hexane $=10 / 90,1 \mathrm{~mL} / \mathrm{min}, 206 \mathrm{~nm}$ ) $\mathrm{t}_{1}=13.8 \mathrm{~min}$ (major), $\mathrm{t}_{2}$ $=15.6 \mathrm{~min}$ (minor). 

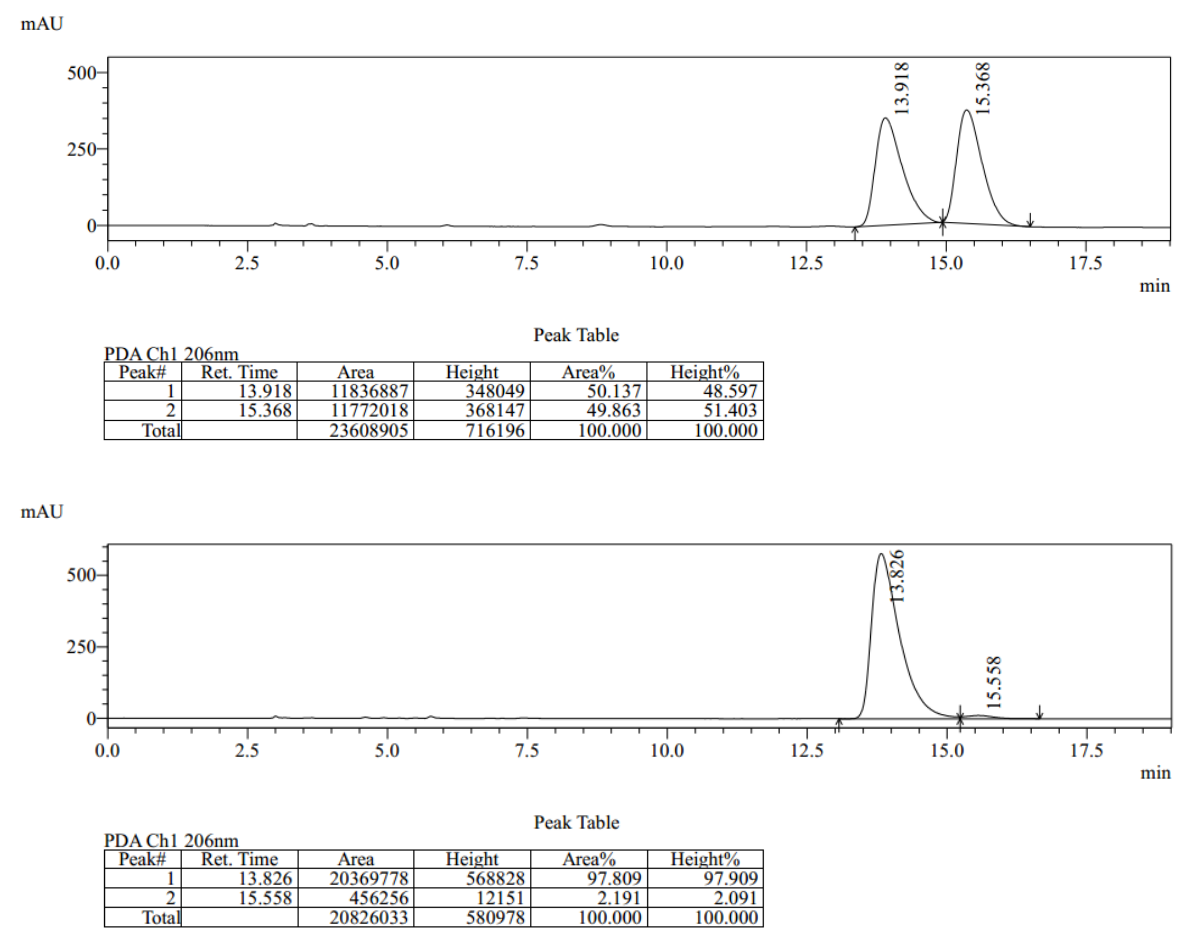

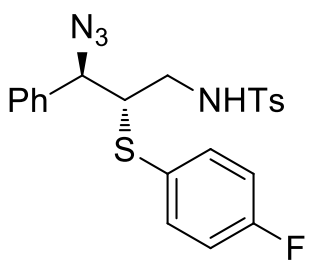

$N$-((2S,3R)-3-Azido-2-((4-fluorophenyl)thio)-3-phenylpropyl)-4-methylbenzenesu Ifonamide (3p): Flash column chromatography (eluent: PE/EtOAc $=15 / 1$ to 5/1, v/v) to afford $\mathbf{3 p}$ as a white solid. $43.3 \mathrm{mg}, 95 \%$ yield, $97 \%$ ee with $>99: 1 d r$. mp 102.3 $103.7{ }^{\circ} \mathrm{C} .[\alpha]^{25} \mathrm{D}=-15.3\left(\mathrm{c}=0.2, \mathrm{CHCl}_{3}\right) .{ }^{1} \mathrm{H} \mathrm{NMR}\left(400 \mathrm{MHz}, \mathrm{CDCl}_{3}\right) \delta 7.67(\mathrm{~d}, J=$ $8.1 \mathrm{~Hz}, 2 \mathrm{H}), 7.37-7.32(\mathrm{~m}, 3 \mathrm{H}), 7.28(\mathrm{~d}, J=8.1 \mathrm{~Hz}, 2 \mathrm{H}), 7.18-7.13(\mathrm{~m}, 2 \mathrm{H}), 7.13$ $-7.08(\mathrm{~m}, 2 \mathrm{H}), 6.87(\mathrm{t}, J=8.6 \mathrm{~Hz}, 2 \mathrm{H}), 5.23(\mathrm{t}, J=6.0 \mathrm{~Hz}, 1 \mathrm{H}), 4.55(\mathrm{~d}, J=7.7 \mathrm{~Hz}$, $1 \mathrm{H}), 3.31(\mathrm{ddd}, J=13.2,7.1,4.6 \mathrm{~Hz}, 1 \mathrm{H}), 3.21(\mathrm{td}, J=7.7,4.6 \mathrm{~Hz}, 1 \mathrm{H}), 3.14-3.05$ (m, 1H), $2.44(\mathrm{~s}, 3 \mathrm{H}) .{ }^{13} \mathrm{C}$ NMR $\left(101 \mathrm{MHz}, \mathrm{CDCl}_{3}\right) \delta 162.87(\mathrm{~d}, J=249.3 \mathrm{~Hz})$, $143.78,136.77,136.41,135.74(\mathrm{~d}, J=8.3 \mathrm{~Hz}), 129.89,129.03,128.95,127.70$, $127.63,127.27,116.41(\mathrm{~d}, J=21.9 \mathrm{~Hz}), 67.48,55.92,43.31,21.64 .{ }^{19} \mathrm{~F}$ NMR $(376$ $\left.\mathrm{MHz}, \mathrm{CDCl}_{3}\right) \delta$-112.37. HR-ESI-MS $\mathrm{m} / z$ calcd. for $\mathrm{C}_{22} \mathrm{H}_{21} \mathrm{FN}_{4} \mathrm{NaO}_{2} \mathrm{~S}_{2}[\mathrm{M}+\mathrm{Na}]^{+}$: 479.0982, found: 472.0985. HPLC (Daicel Chiralpak OJ-H column, $i-\mathrm{PrOH} /$ hexane $=$ 20/80, $1 \mathrm{~mL} / \mathrm{min}, 225 \mathrm{~nm}$ ) $\mathrm{t}_{1}=17.8 \mathrm{~min}$ (major), $\mathrm{t}_{2}=21.9 \mathrm{~min}$ (minor). 
$\mathrm{mAU}$
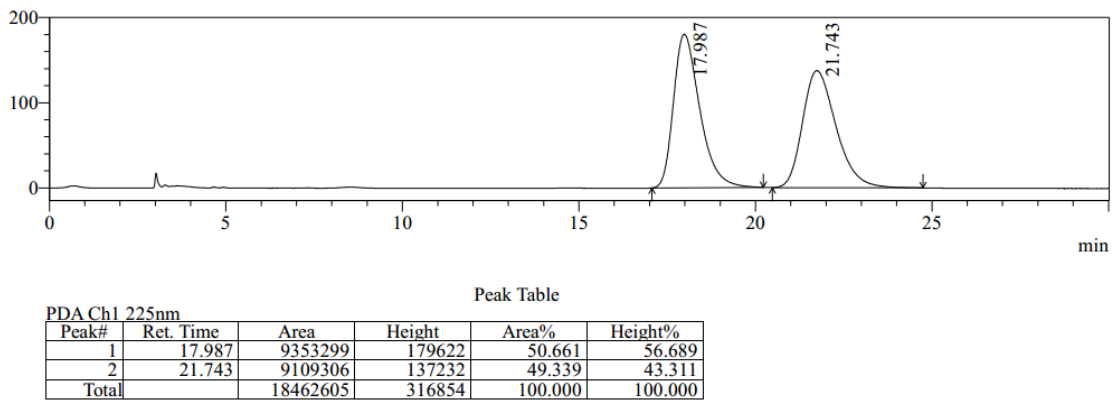

$\mathrm{mAU}$
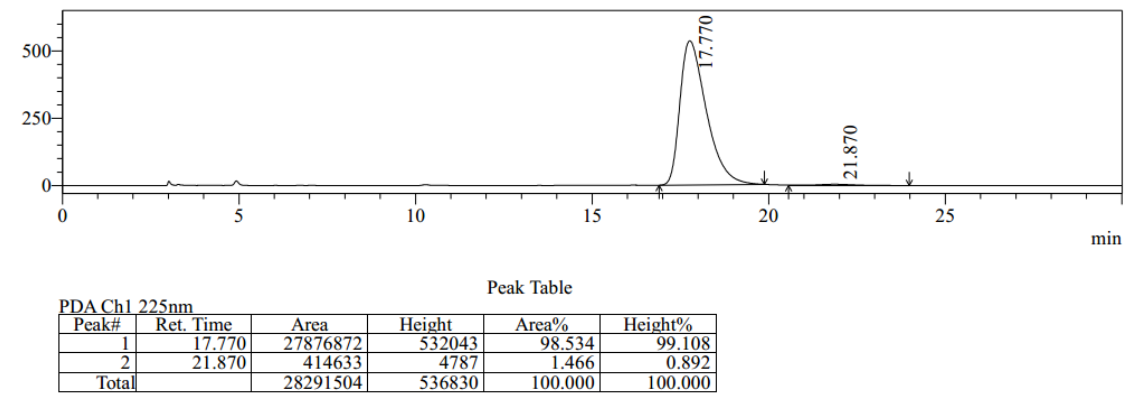

NHTs

$N$-((2S,3R)-3-Azido-2-(hexylthio)-3-(p-tolyl)propyl)-4-methylbenzenesulfonamide

(3q): Flash column chromatography (eluent: PE/EtOAc $=15 / 1$ to $5 / 1, \mathrm{v} / \mathrm{v}$ ) to afford 3q as a colorless oil. $42.3 \mathrm{mg}, 92 \%$ yield, 94\% ee with $>99: 1 d r .[\alpha]^{25} \mathrm{D}=-105.2(\mathrm{c}=$ $\left.0.2, \mathrm{CHCl}_{3}\right) .{ }^{1} \mathrm{H} \mathrm{NMR}\left(400 \mathrm{MHz}, \mathrm{CDCl}_{3}\right) \delta 7.74(\mathrm{~d}, J=8.2 \mathrm{~Hz}, 2 \mathrm{H}), 7.31(\mathrm{~d}, J=8.2$ $\mathrm{Hz}, 2 \mathrm{H}), 7.17(\mathrm{~d}, J=8.0 \mathrm{~Hz}, 2 \mathrm{H}), 7.12(\mathrm{~d}, J=8.0 \mathrm{~Hz}, 2 \mathrm{H}), 5.17(\mathrm{dd}, J=6.9,5.5 \mathrm{~Hz}$, $1 \mathrm{H}), 4.46(\mathrm{~d}, J=8.4 \mathrm{~Hz}, 1 \mathrm{H}), 3.42-3.29(\mathrm{~m}, 1 \mathrm{H}), 3.04-2.95(\mathrm{~m}, 1 \mathrm{H}), 2.44(\mathrm{~s}, 3 \mathrm{H})$, $2.35(\mathrm{~s}, 3 \mathrm{H}), 2.20-2.00(\mathrm{~m}, 2 \mathrm{H}), 1.36-1.19(\mathrm{~m}, 5 \mathrm{H}), 1.19-1.06(\mathrm{~m}, 4 \mathrm{H}), 0.86(\mathrm{t}, J$ $=7.2 \mathrm{~Hz}, 3 \mathrm{H}) .{ }^{13} \mathrm{C} \mathrm{NMR}\left(101 \mathrm{MHz}, \mathrm{CDCl}_{3}\right) \delta 143.66,138.75,136.90,133.86,129.86$, 129.51, 127.63, 127.26, 68.26, 51.10, 43.86, 32.02, 31.30, 29.35, 28.41, 22.56, 21.63, 21.30, 14.10. HR-ESI-MS $\mathrm{m} / z$ calcd. for $\mathrm{C}_{23} \mathrm{H}_{31} \mathrm{~N}_{4} \mathrm{O}_{2} \mathrm{~S}_{2}[\mathrm{M}-\mathrm{H}]^{-}:$459.1894, found: 459.1905. HPLC (Daicel Chiralpak OD-H column, $i$-PrOH $/$ hexane $=3 / 97,1 \mathrm{~mL} / \mathrm{min}$, $223 \mathrm{~nm}$ ) $\mathrm{t}_{1}=16.7 \mathrm{~min}$ (minor), $\mathrm{t}_{2}=18.4 \mathrm{~min}$ (major).

$\mathrm{mAU}$
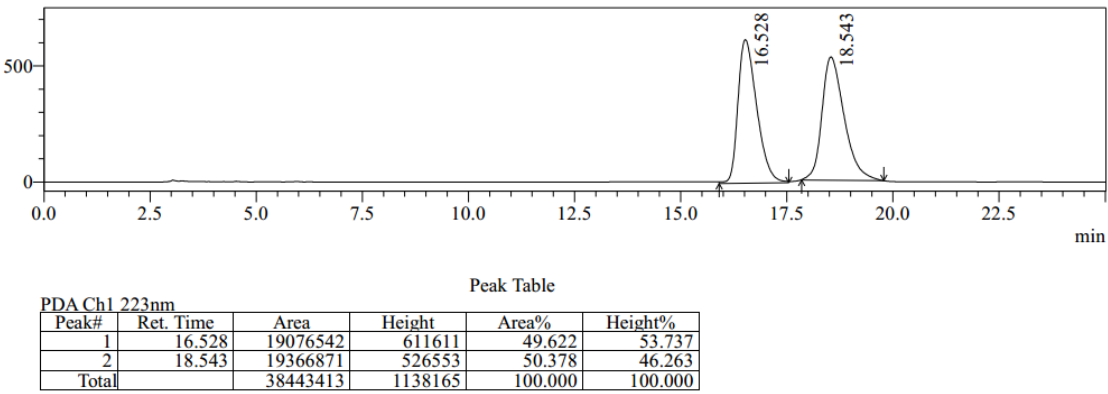
$\mathrm{mAU}$
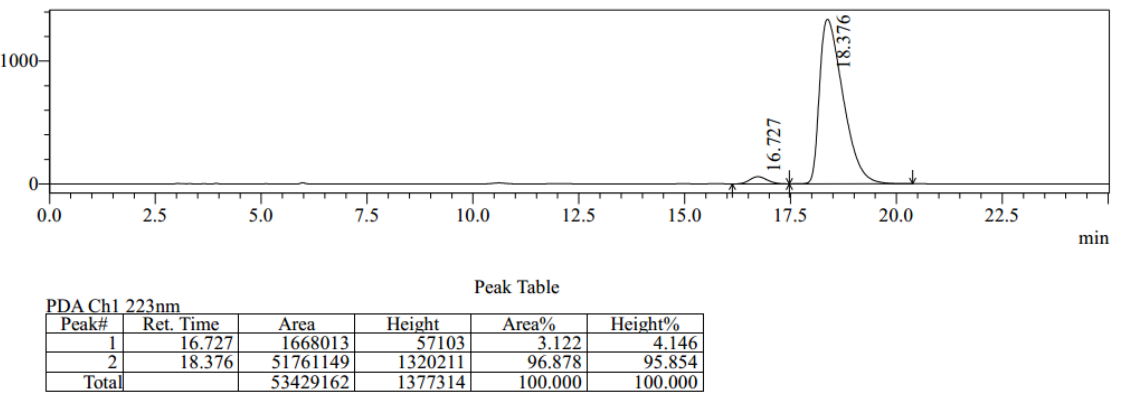

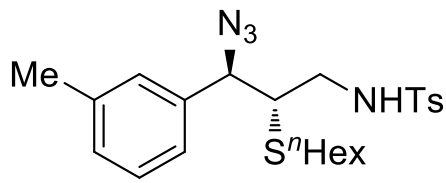

$N$-((2S,3R)-3-Azido-2-(hexylthio)-3-(m-tolyl)propyl)-4-methylbenzenesulfonamid e (3r): Flash column chromatography (eluent: PE/EtOAc $=15 / 1$ to $5 / 1, \mathrm{v} / \mathrm{v}$ ) to afford 3r as a colorless oil. $42.8 \mathrm{mg}, 93 \%$ yield, 94\% ee with $>99: 1 d r .[\alpha]^{25} \mathrm{D}=-102.5(\mathrm{c}=$ 0.2, $\left.\mathrm{CHCl}_{3}\right) .{ }^{1} \mathrm{H} \mathrm{NMR}\left(400 \mathrm{MHz}, \mathrm{CDCl}_{3}\right) \delta 7.79-7.70(\mathrm{~m}, 2 \mathrm{H}), 7.31(\mathrm{~d}, J=8.0 \mathrm{~Hz}$, 2H), $7.27-7.22(\mathrm{~m}, 1 \mathrm{H}), 7.15(\mathrm{~d}, J=7.4 \mathrm{~Hz}, 1 \mathrm{H}), 7.03(\mathrm{~d}, J=7.4 \mathrm{~Hz}, 2 \mathrm{H}), 5.16(\mathrm{dd}$, $J=7.4,5.2 \mathrm{~Hz}, 1 \mathrm{H}), 4.45(\mathrm{~d}, J=7.8 \mathrm{~Hz}, 1 \mathrm{H}), 3.36$ (ddd, $J=13.2,7.4,4.7 \mathrm{~Hz}, 1 \mathrm{H}$ ), $3.05-2.92(\mathrm{~m}, 1 \mathrm{H}), 2.78(\mathrm{td}, J=7.8,4.7 \mathrm{~Hz}, 1 \mathrm{H}), 2.43(\mathrm{~s}, 3 \mathrm{H}), 2.36(\mathrm{~s}, 3 \mathrm{H}), 2.19-$ $2.02(\mathrm{~m}, 2 \mathrm{H}), 1.35-1.21(\mathrm{~m}, 4 \mathrm{H}), 1.19-1.09(\mathrm{~m}, 4 \mathrm{H}), 0.86(\mathrm{t}, J=7.2 \mathrm{~Hz}, 3 \mathrm{H}) .{ }^{13} \mathrm{C}$ NMR $\left(101 \mathrm{MHz}, \mathrm{CDCl}_{3}\right) \delta 143.65,138.58,136.96,136.88,129.86,129.68,128.72$, 128.34, 127.27, 124.79, 68.59, 51.09, 43.85, 32.06, 31.32, 29.38, 28.42, 22.57, 21.64, 21.57, 14.10. HR-ESI-MS $\mathrm{m} / z$ calcd. for $\mathrm{C}_{23} \mathrm{H}_{31} \mathrm{~N}_{4} \mathrm{O}_{2} \mathrm{~S}_{2}$ [M-H] : 459.1894, found: 459.1900. HPLC (Daicel Chiralpak OD-H column, $i$-PrOH/hexane $=3 / 97,1 \mathrm{~mL} / \mathrm{min}$, $223 \mathrm{~nm}$ ) $\mathrm{t}_{1}=15.7 \min$ (minor), $\mathrm{t}_{2}=16.9 \min$ (major).

$\mathrm{mAU}$

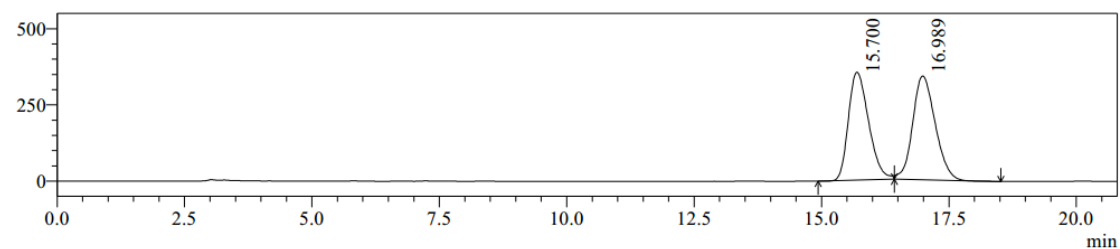

Peak Table

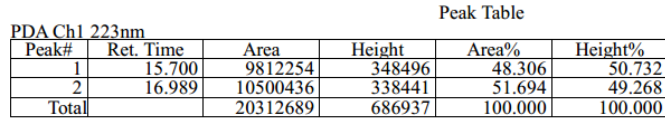

$\mathrm{mAU}$

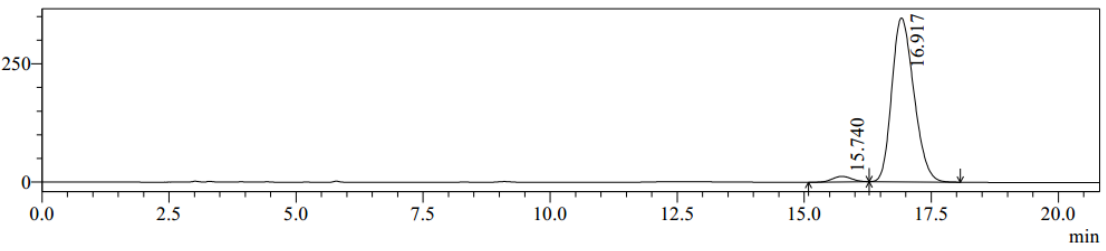

Peak Table

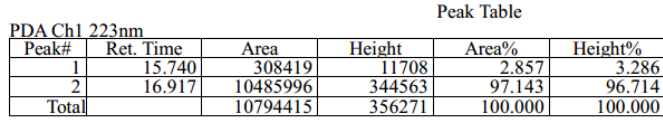


$\overbrace{\mathrm{NHTS}_{\mathrm{s}^{n} \mathrm{Hex}}}^{\mathrm{MeO}_{3}}$

$N$-((2S,3R)-3-Azido-2-(hexylthio)-3-(3-methoxyphenyl)propyl)-4-methylbenzenes ulfonamide (3s): Flash column chromatography (eluent: PE/EtOAc $=15 / 1$ to 5/1, v/v) to afford $3 \mathrm{~s}$ as a colorless oil. $41.9 \mathrm{mg}, 88 \%$ yield, $96 \%$ ee with $32: 1 \mathrm{dr}$. $[\alpha]^{25} \mathrm{D}=$ -101.6 (c = 0.2, $\left.\mathrm{CHCl}_{3}\right) .{ }^{1} \mathrm{H} \mathrm{NMR}\left(400 \mathrm{MHz}, \mathrm{CDCl}_{3}\right) \delta 7.72(\mathrm{~d}, J=8.2 \mathrm{~Hz}, 2 \mathrm{H}), 7.34$ $-7.27(\mathrm{~m}, 3 \mathrm{H}), 6.88(\mathrm{~d}, J=8.2 \mathrm{~Hz}, 1 \mathrm{H}), 6.83$ (d, $J=7.6 \mathrm{~Hz}, 1 \mathrm{H}), 6.79$ (s, 1H), 5.13 $(\mathrm{dd}, J=7.2,5.3 \mathrm{~Hz}, 1 \mathrm{H}), 4.47(\mathrm{~d}, J=7.8 \mathrm{~Hz}, 1 \mathrm{H}), 3.81(\mathrm{~s}, 3 \mathrm{H}), 3.35(\mathrm{ddd}, J=12.7$, 7.2, $4.7 \mathrm{~Hz}, 1 \mathrm{H}), 3.05-2.94(\mathrm{~m}, 1 \mathrm{H}), 2.78(\mathrm{td}, J=7.8,4.7 \mathrm{~Hz}, 1 \mathrm{H}), 2.44(\mathrm{~s}, 3 \mathrm{H}), 2.20$ $-2.06(\mathrm{~m}, 2 \mathrm{H}), 1.38-1.28(\mathrm{~m}, 2 \mathrm{H}), 1.28-1.10(\mathrm{~m}, 6 \mathrm{H}), 0.86(\mathrm{t}, J=7.1 \mathrm{~Hz}, 3 \mathrm{H}) .{ }^{13} \mathrm{C}$ NMR $\left(101 \mathrm{MHz}, \mathrm{CDCl}_{3}\right) \delta 159.95,143.72,138.52,136.94,129.94,129.90,127.28$, $119.98,114.18,113.50,68.50,55.42,51.14,43.82,32.16,31.35,29.43,28.46,22.59$, 21.66, 14.12. HR-ESI-MS $\mathrm{m} / z$ calcd. for $\mathrm{C}_{23} \mathrm{H}_{31} \mathrm{~N}_{4} \mathrm{O}_{3} \mathrm{~S}_{2}[\mathrm{M}-\mathrm{H}]^{-}: 475.1843$, found: 475.1847. HPLC (Daicel Chiralpak OD-H column, $i$-PrOH/hexane $=5 / 95,1 \mathrm{~mL} / \mathrm{min}$, $223 \mathrm{~nm}$ ) $\mathrm{t}_{1}=14.5 \mathrm{~min}$ (minor), $\mathrm{t}_{2}=19.6 \mathrm{~min}$ (major).

$\mathrm{mAU}$
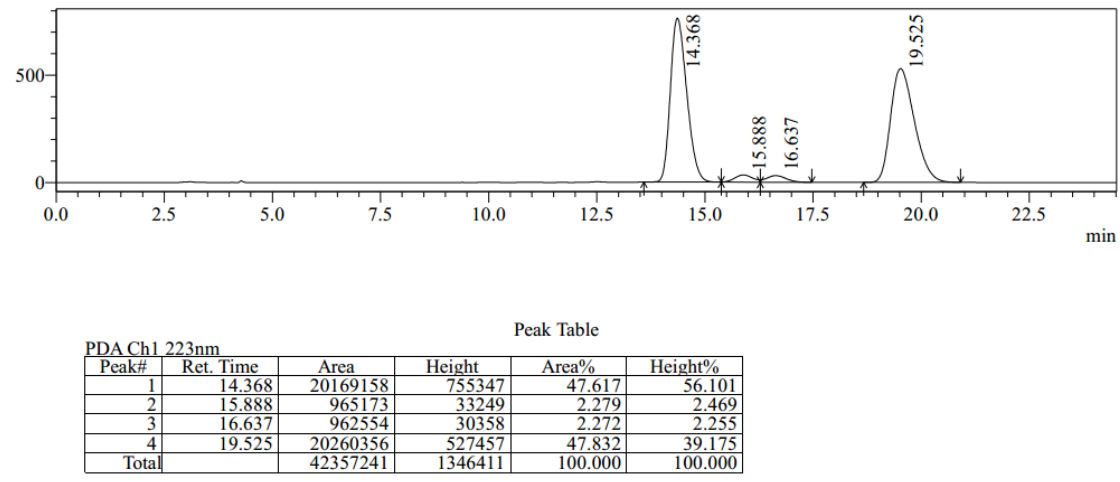

$\mathrm{mAU}$
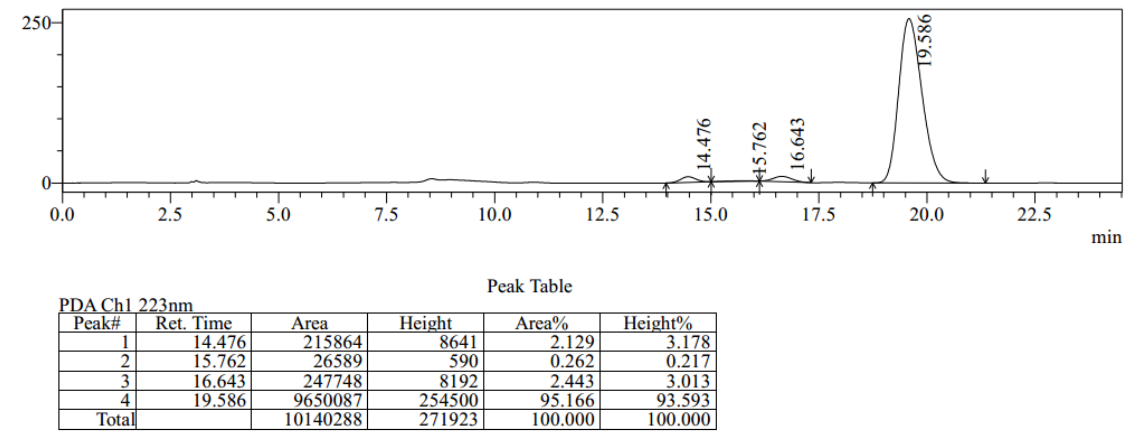

$\overbrace{\mathrm{s}_{\mathrm{S}^{n} \mathrm{Hex}}}^{\mathrm{N}_{3}}$

$N$-((2S,3R)-3-Azido-3-(4-chlorophenyl)-2-(hexylthio)propyl)-4-methylbenzenesulf onamide (3t): Flash column chromatography (eluent: PE/EtOAc $=15 / 1$ to $5 / 1$, v/v) to 
afford 3t as a colorless oil. $39.4 \mathrm{mg}, 82 \%$ yield, 95\% ee with $>99: 1 d r .[\alpha]^{25} \mathrm{D}=-76.3$ $\left(\mathrm{c}=0.2, \mathrm{CHCl}_{3}\right) .{ }^{1} \mathrm{H} \mathrm{NMR}\left(400 \mathrm{MHz}, \mathrm{CDCl}_{3}\right) \delta 7.74(\mathrm{~d}, J=8.3 \mathrm{~Hz}, 2 \mathrm{H}), 7.33(\mathrm{t}, J=$ $8.9 \mathrm{~Hz}, 4 \mathrm{H}), 7.21(\mathrm{~d}, J=8.3 \mathrm{~Hz}, 2 \mathrm{H}), 5.20(\mathrm{t}, J=5.9 \mathrm{~Hz}, 1 \mathrm{H}), 4.49(\mathrm{~d}, J=8.4 \mathrm{~Hz}$, $1 \mathrm{H}), 3.37-3.21(\mathrm{~m}, 1 \mathrm{H}), 3.07-2.94(\mathrm{~m}, 1 \mathrm{H}), 2.77(\mathrm{dd}, J=12.6,7.6 \mathrm{~Hz}, 1 \mathrm{H}), 2.44(\mathrm{~s}$, $3 \mathrm{H}), 2.25-2.03(\mathrm{~m}, 2 \mathrm{H}), 1.36-1.10(\mathrm{~m}, 8 \mathrm{H}), 0.86(\mathrm{t}, J=7.1 \mathrm{~Hz}, 3 \mathrm{H}) .{ }^{13} \mathrm{C} \mathrm{NMR}$ $\left(101 \mathrm{MHz}, \mathrm{CDCl}_{3}\right) \delta 143.78,136.86,135.61,134.78,129.89,129.17,129.03,127.27$, 67.60, 51.29, 43.92, 32.30, 31.32, 29.37, 28.39, 22.57, 21.64, 14.09. HR-ESI-MS m/z calcd. for $\mathrm{C}_{22} \mathrm{H}_{28} \mathrm{ClN}_{4} \mathrm{O}_{2} \mathrm{~S}_{2}[\mathrm{M}-\mathrm{H}]^{-}$: 479.1348, found: 479.1355. HPLC (Daicel Chiralpak OD-H column, $i$-PrOH/hexane $=4 / 96,1 \mathrm{~mL} / \mathrm{min}, 223 \mathrm{~nm}) \mathrm{t}_{1}=16.7 \mathrm{~min}$ (minor), $\mathrm{t}_{2}=18.7 \mathrm{~min}$ (major).
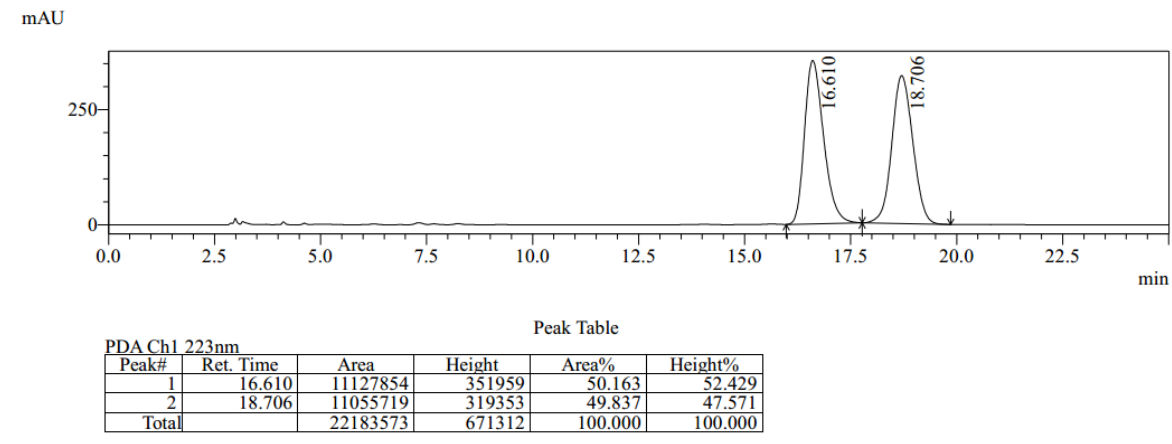

$\mathrm{mAU}$
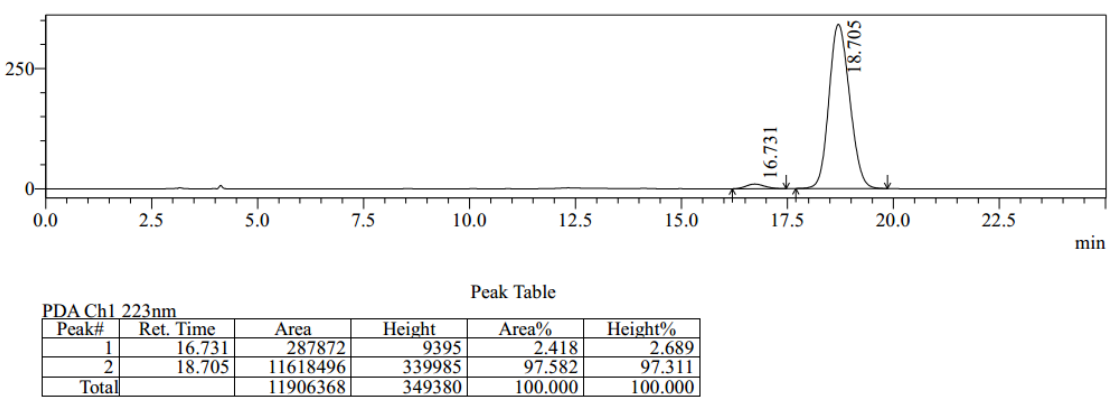

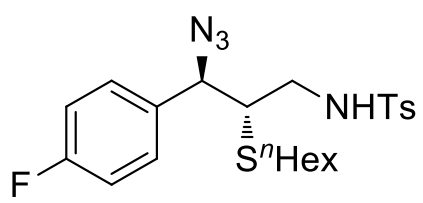

$N$-((2S,3R)-3-Azido-3-(4-fluorophenyl)-2-(hexylthio)propyl)-4-methylbenzenesulf onamide (3u): Flash column chromatography (eluent: PE/EtOAc $=15 / 1$ to $5 / 1$, v/v) to afford $3 \mathbf{u}$ as a colorless oil. $31.6 \mathrm{mg}, 68 \%$ yield, $94 \%$ ee with $>99: 1 d r$. $[\alpha]^{25}{ }_{\mathrm{D}}=$ -90.6 (c = 0.2, $\left.\mathrm{CHCl}_{3}\right) .{ }^{1} \mathrm{H} \mathrm{NMR}\left(400 \mathrm{MHz}, \mathrm{CDCl}_{3}\right) \delta 7.75(\mathrm{~d}, J=7.8 \mathrm{~Hz}, 2 \mathrm{H}), 7.33$ $(\mathrm{d}, J=7.8 \mathrm{~Hz}, 2 \mathrm{H}), 7.29-7.22(\mathrm{~m}, 2 \mathrm{H}), 7.07(\mathrm{dd}, J=8.4,7.4 \mathrm{~Hz}, 2 \mathrm{H}), 5.17(\mathrm{t}, J=$ $5.9 \mathrm{~Hz}, 1 \mathrm{H}), 4.49$ (d, $J=8.5 \mathrm{~Hz}, 1 \mathrm{H}), 3.39-3.26(\mathrm{~m}, 1 \mathrm{H}), 3.08-2.97(\mathrm{~m}, 1 \mathrm{H}), 2.77$ $(\mathrm{dd}, J=12.1,7.4 \mathrm{~Hz}, 1 \mathrm{H}), 2.44(\mathrm{~s}, 3 \mathrm{H}), 2.21-2.04(\mathrm{~m}, 2 \mathrm{H}), 1.36-1.07(\mathrm{~m}, 8 \mathrm{H})$, $0.86(\mathrm{t}, J=7.1 \mathrm{~Hz}, 1 \mathrm{H}) .{ }^{13} \mathrm{C} \mathrm{NMR}\left(101 \mathrm{MHz}, \mathrm{CDCl}_{3}\right) \delta 162.88(\mathrm{~d}, J=248.1 \mathrm{~Hz})$, $143.79,136.87,132.94$ (d, $J=3.1 \mathrm{~Hz}), 129.91,129.63,129.55,127.29,115.85$ (d, $J=$ $21.7 \mathrm{~Hz}), 67.65,51.42,43.96,32.25,31.33,29.38,28.41,22.58,21.66,14.10 .{ }^{19} \mathrm{~F}$ NMR $\left(376 \mathrm{MHz}, \mathrm{CDCl}_{3}\right) \delta-112.45$. HR-ESI-MS $\mathrm{m} / z$ calcd. for $\mathrm{C}_{22} \mathrm{H}_{29} \mathrm{FN}_{4} \mathrm{NaO}_{2} \mathrm{~S}_{2}$ 
$[\mathrm{M}+\mathrm{Na}]^{+}:$487.1608, found: 487.1596. HPLC (Daicel Chiralpak IA column, $i$-PrOH/hexane $=5 / 95,1 \mathrm{~mL} / \mathrm{min}, 223 \mathrm{~nm}) \mathrm{t}_{1}=17.7 \mathrm{~min}$ (minor), $\mathrm{t}_{2}=20.1 \mathrm{~min}$ (major).

$\mathrm{mAU}$
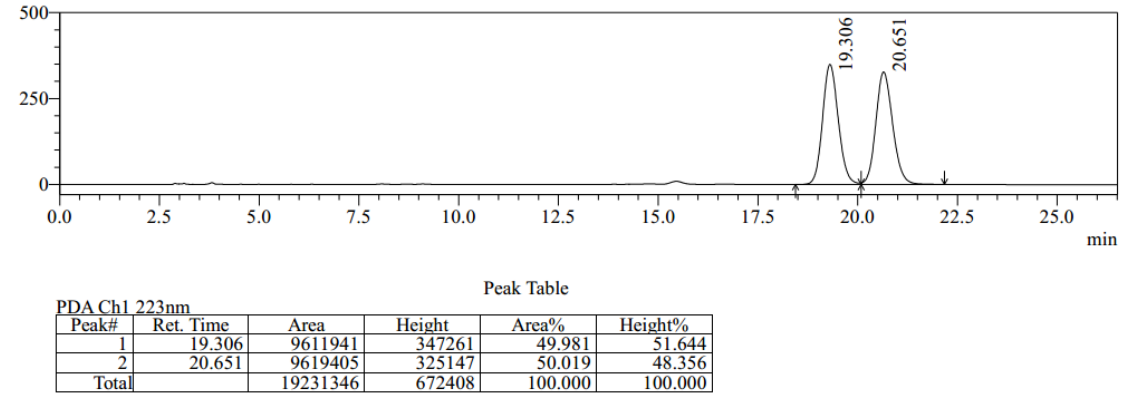

$\mathrm{mAU}$

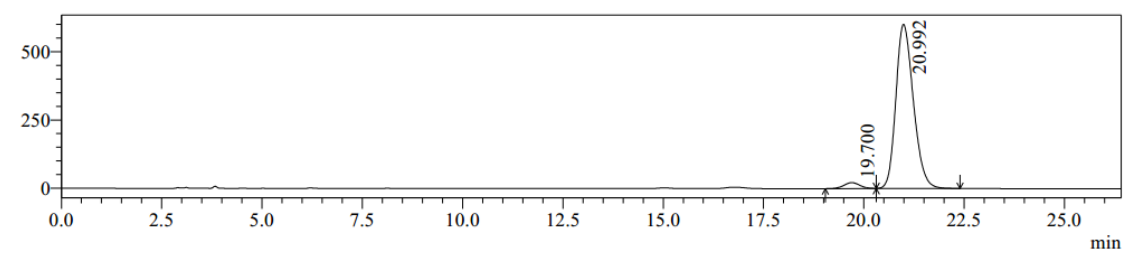

Peak Table

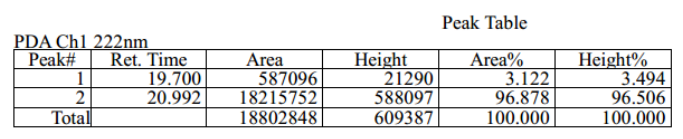

$\overbrace{\mathrm{s}^{n} \mathrm{Hex}}^{\mathrm{NHTS}}$

$N$-((2S,3R)-3-Azido-3-(4-bromophenyl)-2-(hexylthio)propyl)-4-methylbenzenesul

fonamide (3v): Flash column chromatography (eluent: PE/EtOAc $=15 / 1$ to $5 / 1, \mathrm{v} / \mathrm{v}$ ) to afford $3 \mathrm{v}$ as a colorless oil. $43.0 \mathrm{mg}, 82 \%$ yield, $96 \%$ ee with $>99: 1 \mathrm{dr}$. $[\alpha]^{25} \mathrm{D}=$ -97.5 (c = 0.2, $\left.\mathrm{CHCl}_{3}\right) .{ }^{1} \mathrm{H} \mathrm{NMR}\left(400 \mathrm{MHz}, \mathrm{CDCl}_{3}\right) \delta 7.74(\mathrm{~d}, J=7.5 \mathrm{~Hz}, 2 \mathrm{H}), 7.50$ $(\mathrm{d}, J=7.5 \mathrm{~Hz}, 2 \mathrm{H}), 7.32(\mathrm{~d}, J=7.8 \mathrm{~Hz}, 2 \mathrm{H}), 7.15(\mathrm{~d}, J=7.8 \mathrm{~Hz}, 2 \mathrm{H}), 5.21(\mathrm{t}, J=6.0$ $\mathrm{Hz}, 1 \mathrm{H}), 4.48(\mathrm{~d}, J=8.4 \mathrm{~Hz}, 1 \mathrm{H}), 3.34-3.24(\mathrm{~m}, 1 \mathrm{H}), 3.07-2.96(\mathrm{~m}, 1 \mathrm{H}), 2.77(\mathrm{dd}$, $J=12.3,7.3 \mathrm{~Hz}, 1 \mathrm{H}), 2.44(\mathrm{~s}, 3 \mathrm{H}), 2.23-2.03(\mathrm{~m}, 2 \mathrm{H}), 1.37-1.20(\mathrm{~m}, 4 \mathrm{H}), 1.20-$ $1.02(\mathrm{~m}, 4 \mathrm{H}), 0.87(\mathrm{t}, J=6.9 \mathrm{~Hz}, 3 \mathrm{H}) .{ }^{13} \mathrm{C} \mathrm{NMR}\left(101 \mathrm{MHz}, \mathrm{CDCl}_{3}\right) \delta 143.79,136.80$, 136.12, 131.99, 129.90, 129.46, 127.26, 122.93, 67.64, 51.21, 43.88, 32.31, 31.32, 29.36, 28.40, 22.58, 21.66, 14.12. HR-ESI-MS $\mathrm{m} / z$ calcd. for $\mathrm{C}_{22} \mathrm{H}_{28} \mathrm{BrN}_{4} \mathrm{O}_{2} \mathrm{~S}_{2}[\mathrm{M}-\mathrm{H}]^{-}:$ 523.0843, found: 523.0846. HPLC (Daicel Chiralpak OD-H column, $i-\mathrm{PrOH} /$ hexane $=4 / 96,1 \mathrm{~mL} / \mathrm{min}, 223 \mathrm{~nm}$ ) $\mathrm{t}_{1}=17.6 \mathrm{~min}$ (minor), $\mathrm{t}_{2}=19.3 \mathrm{~min}$ (major). 

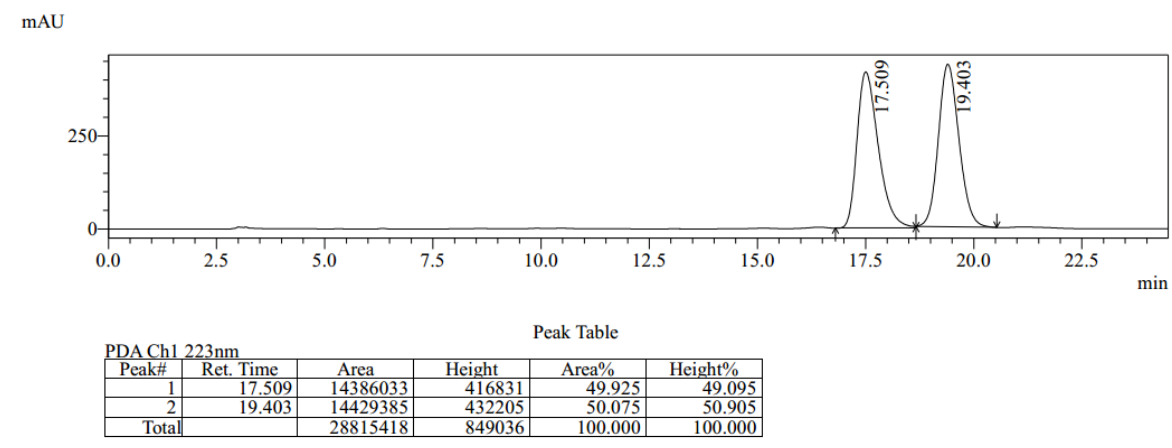

$\mathrm{mAU}$
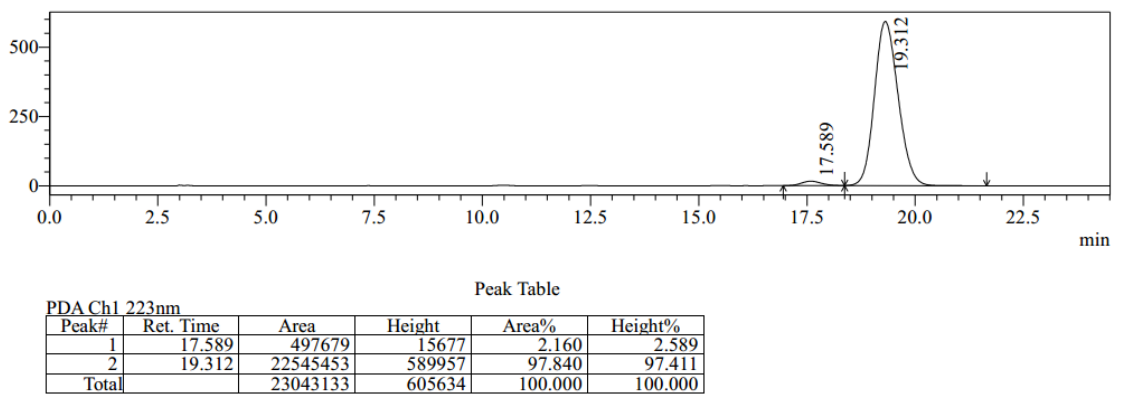

$\underbrace{N_{3}}_{\underbrace{N H T s}_{\hat{s}^{n} \mathrm{Hex}}}$

$N$-((2S,3R)-3-Azido-3-(2-fluorophenyl)-2-(hexylthio)propyl)-4-methylbenzenesulf onamide (3w): Flash column chromatography (eluent: PE/EtOAc $=15 / 1$ to $5 / 1, \mathrm{v} / \mathrm{v}$ ) to afford $3 \mathrm{w}$ as a colorless oil. $40.8 \mathrm{mg}, 88 \%$ yield, $85 \%$ ee with $>99: 1 d r .[\alpha]^{25} \mathrm{D}=$ -59.2 (c = 0.2, $\left.\mathrm{CHCl}_{3}\right) .{ }^{1} \mathrm{H} \mathrm{NMR}\left(400 \mathrm{MHz}, \mathrm{CDCl}_{3}\right) \delta 7.70(\mathrm{~d}, J=8.0 \mathrm{~Hz}, 2 \mathrm{H}), 7.38-$ $7.27(\mathrm{~m}, 4 \mathrm{H}), 7.18(\mathrm{t}, J=7.5 \mathrm{~Hz}, 1 \mathrm{H}), 7.08(\mathrm{dd}, J=9.7,8.9 \mathrm{~Hz}, 1 \mathrm{H}), 5.14(\mathrm{dd}, J=7.4$, $5.0 \mathrm{~Hz}, 1 \mathrm{H}), 4.89(\mathrm{~d}, J=7.5 \mathrm{~Hz}, 1 \mathrm{H}), 3.35-3.23(\mathrm{~m}, 1 \mathrm{H}), 3.07-2.97(\mathrm{~m}, 1 \mathrm{H}), 2.89$ $(\mathrm{td}, J=7.5,4.6 \mathrm{~Hz}, 1 \mathrm{H}), 2.43(\mathrm{~s}, 3 \mathrm{H}), 2.35-2.15(\mathrm{~m}, 2 \mathrm{H}), 1.48-1.31(\mathrm{~m}, 2 \mathrm{H}), 1.31$ $-1.09(\mathrm{~m}, 6 \mathrm{H}), 0.87(\mathrm{t}, J=7.0 \mathrm{~Hz}, 3 \mathrm{H}) .{ }^{13} \mathrm{C} \mathrm{NMR}\left(101 \mathrm{MHz}, \mathrm{CDCl}_{3}\right) \delta 160.29(\mathrm{~d}, J=$ $247.1 \mathrm{~Hz}), 143.69,136.89,130.50(\mathrm{~d}, J=8.4 \mathrm{~Hz}), 129.89,128.79(\mathrm{~d}, J=3.4 \mathrm{~Hz})$, 127.18, 124.69 (d, $J=3.4 \mathrm{~Hz}), 124.40(\mathrm{~d}, J=13.0 \mathrm{~Hz}), 115.92(\mathrm{~d}, J=21.9 \mathrm{~Hz}), 62.09$, 50.26, 43.43, 32.00, 31.32, 29.46, 28.41, 22.56, 21.63, 14.10. ${ }^{19} \mathrm{~F}$ NMR (376 MHz, $\left.\mathrm{CDCl}_{3}\right) \delta-117.15$. HR-ESI-MS $\mathrm{m} / z$ calcd. for $\mathrm{C}_{22} \mathrm{H}_{28} \mathrm{~N}_{4} \mathrm{O}_{2} \mathrm{FS}_{2}[\mathrm{M}-\mathrm{H}]=$ : 463.1643, found: 463.1650. HPLC (Daicel Chiralpak OD-H column, $i$-PrOH/hexane $=5 / 95,1$ $\mathrm{mL} / \mathrm{min}, 223 \mathrm{~nm}$ ) $\mathrm{t}_{1}=9.2 \min$ (minor), $\mathrm{t}_{2}=10.5 \min$ (major). 

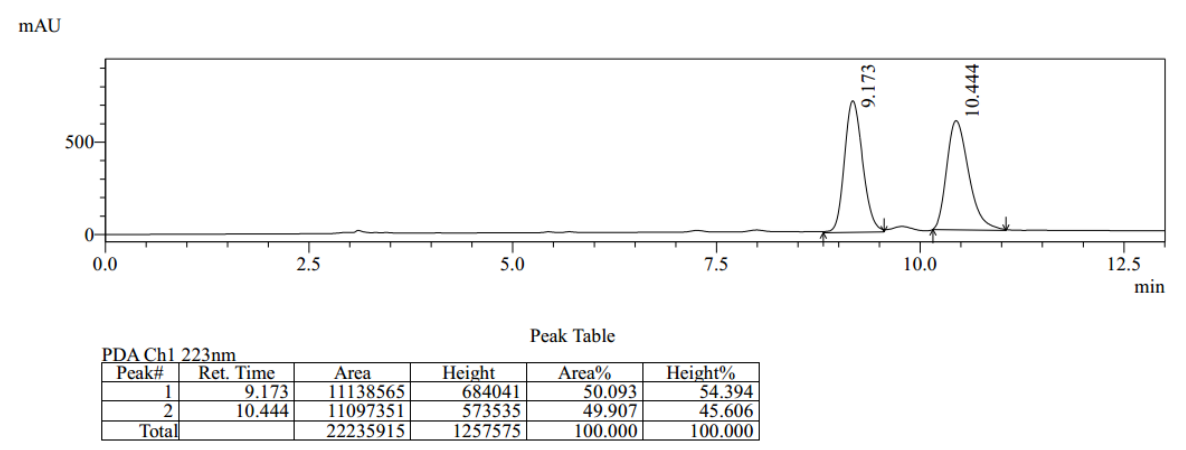

mAU
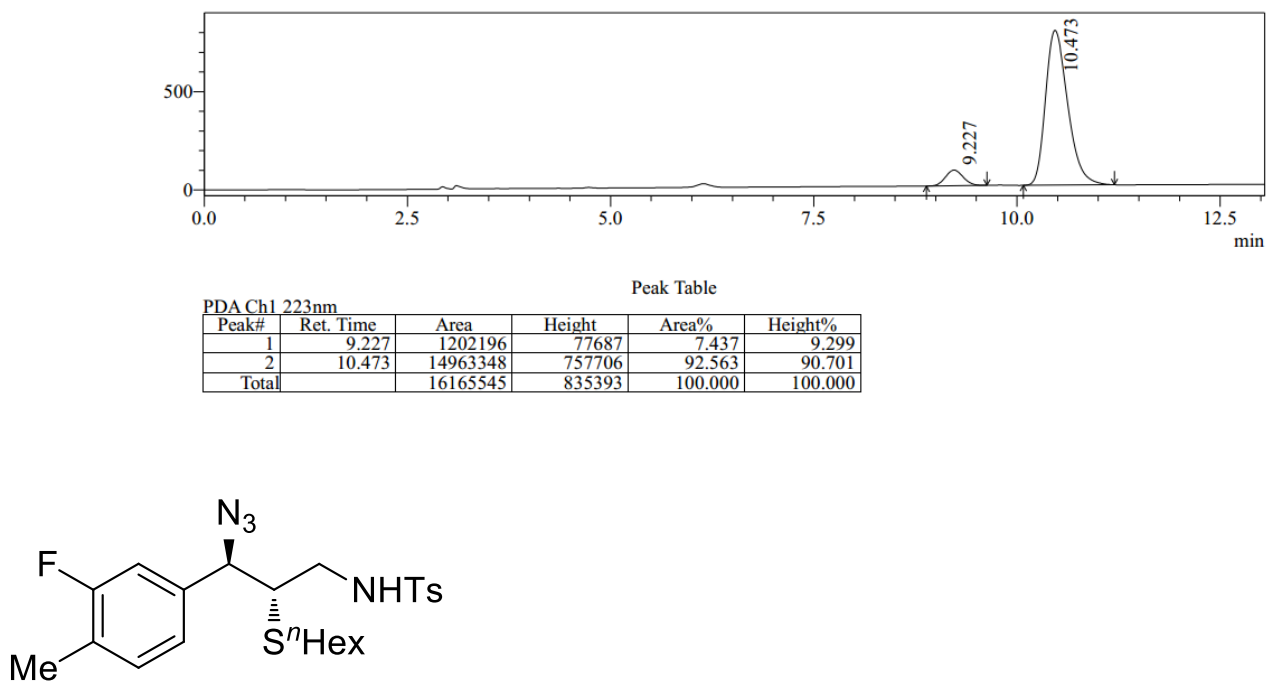

$N$-((2S,3R)-3-Azido-3-(3-fluoro-4-methylphenyl)-2-(hexylthio)propyl)-4-methylbe nzenesulfonamide (3x): Flash column chromatography (eluent: PE/EtOAc $=15 / 1$ to $5 / 1, \mathrm{v} / \mathrm{v}$ ) to afford $\mathbf{3 x}$ as a colorless oil. $37.3 \mathrm{mg}, 78 \%$ yield, $93 \%$ ee with $>99: 1 d r$. $[\alpha]^{25} \mathrm{D}=-73.1\left(\mathrm{c}=0.2, \mathrm{CHCl}_{3}\right) .{ }^{1} \mathrm{H} \mathrm{NMR}\left(400 \mathrm{MHz}, \mathrm{CDCl}_{3}\right) \delta 7.74(\mathrm{~d}, J=8.3 \mathrm{~Hz}$, $2 \mathrm{H}), 7.32(\mathrm{~d}, J=8.3 \mathrm{~Hz}, 2 \mathrm{H}), 7.18(\mathrm{t}, J=7.8 \mathrm{~Hz}, 1 \mathrm{H}), 6.99-6.85(\mathrm{~m}, 2 \mathrm{H}), 5.15(\mathrm{dd}$, $J=7.0,5.6 \mathrm{~Hz}, 1 \mathrm{H}), 4.44(\mathrm{~d}, J=8.0 \mathrm{~Hz}, 1 \mathrm{H}), 3.32(\mathrm{ddd}, J=13.3,7.6,4.8 \mathrm{~Hz}, 1 \mathrm{H})$, $3.06-2.96(\mathrm{~m}, 1 \mathrm{H}), 2.73(\mathrm{td}, J=8.0,4.8 \mathrm{~Hz}, 1 \mathrm{H}), 2.44(\mathrm{~s}, 3 \mathrm{H}), 2.28(\mathrm{~d}, J=1.4 \mathrm{~Hz}$, $3 \mathrm{H}), 2.21-2.08(\mathrm{~m}, 2 \mathrm{H}), 1.37-1.20(\mathrm{~m}, 4 \mathrm{H}), 1.20-1.11(\mathrm{~m}, 4 \mathrm{H}), 0.86(\mathrm{t}, J=7.1 \mathrm{~Hz}$, $3 \mathrm{H}) .{ }^{13} \mathrm{C} \mathrm{NMR}\left(101 \mathrm{MHz}, \mathrm{CDCl}_{3}\right) \delta 161.33(\mathrm{~d}, J=246.3 \mathrm{~Hz}), 143.81,136.93,136.65$ $(\mathrm{d}, J=6.8 \mathrm{~Hz}), 131.86(\mathrm{~d}, J=5.4 \mathrm{~Hz}), 129.92,127.29,125.70(\mathrm{~d}, J=17.3 \mathrm{~Hz})$, $123.24(\mathrm{~d}, J=3.2 \mathrm{~Hz}), 114.29(\mathrm{~d}, J=23.2 \mathrm{~Hz}), 67.72,51.11,43.82,32.18,31.33$, 29.39, 28.43, 22.59, 21.65, 14.52, 14.49, 14.11. ${ }^{19} \mathrm{~F}$ NMR $\left(376 \mathrm{MHz}, \mathrm{CDCl}_{3}\right) \delta$ -115.93. HR-ESI-MS $\mathrm{m} / z$ calcd. for $\mathrm{C}_{23} \mathrm{H}_{30} \mathrm{FN}_{4} \mathrm{O}_{2} \mathrm{~S}_{2}[\mathrm{M}-\mathrm{H}]^{-}: 477.1800$, found: 477.1807. HPLC (Daicel Chiralpak OD-H column, $i$-PrOH/hexane $=5 / 95,1 \mathrm{~mL} / \mathrm{min}$, $223 \mathrm{~nm}$ ) $\mathrm{t}_{1}=9.4 \min$ (minor), $\mathrm{t}_{2}=10.7 \mathrm{~min}$ (major). 
$\mathrm{mAU}$
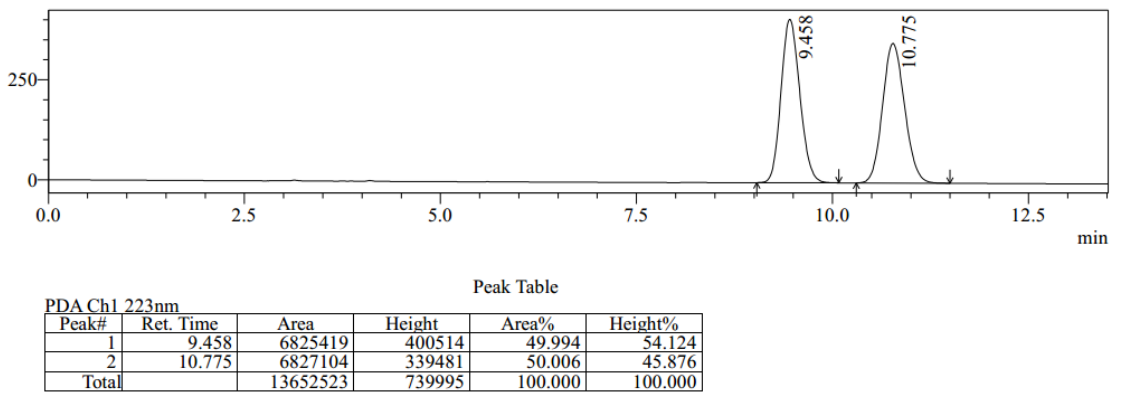

$\mathrm{mAU}$
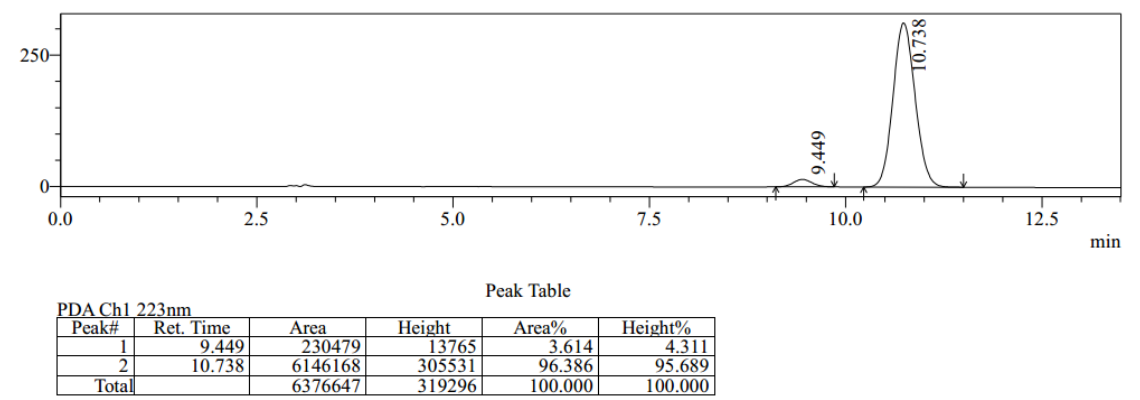

$\overbrace{\underbrace{N}_{\mathbf{s}^{n} \mathrm{Hex}}}^{\mathrm{N}_{3}}$

$N$-((2S,3R)-3-Aazido-2-(hexylthio)-3-(naphthalen-2-yl)propyl)-4-methylbenzenes ulfonamide (3y): Flash column chromatography (eluent: PE/EtOAc $=15 / 1$ to 5/1, v/v) to afford $3 \mathbf{y}$ as a colorless oil. $44.6 \mathrm{mg}$, 90\% yield, 92\% ee with $>99: 1 d r .[\alpha]^{25} \mathrm{D}=$ -114.1 (c = 0.2, $\left.\mathrm{CHCl}_{3}\right) .{ }^{1} \mathrm{H} \mathrm{NMR}\left(400 \mathrm{MHz}, \mathrm{CDCl}_{3}\right) \delta 7.91-7.73(\mathrm{~m}, 3 \mathrm{H}), 7.71(\mathrm{~d}, J$ $=8.1 \mathrm{~Hz}, 3 \mathrm{H}), 7.55-7.47(\mathrm{~m}, 2 \mathrm{H}), 7.35(\mathrm{dd}, J=8.5,1.6 \mathrm{~Hz}, 1 \mathrm{H}), 7.28-7.21(\mathrm{~m}$, $2 \mathrm{H}), 5.27-5.10(\mathrm{~m}, 1 \mathrm{H}), 4.66(\mathrm{~d}, J=8.0 \mathrm{~Hz}, 1 \mathrm{H}), 3.44-3.33(\mathrm{~m}, 1 \mathrm{H}), 3.12-3.03$ (m, 1H), $2.91(\mathrm{td}, J=8.0,4.7 \mathrm{~Hz}, 1 \mathrm{H}), 2.41(\mathrm{~s}, 3 \mathrm{H}), 2.18-2.05(\mathrm{~m}, 2 \mathrm{H}), 1.34-1.19$ $(\mathrm{m}, 3 \mathrm{H}), 1.20-0.96(\mathrm{~m}, 6 \mathrm{H}), 0.80(\mathrm{t}, J=7.2 \mathrm{~Hz}, 3 \mathrm{H}) .{ }^{13} \mathrm{C} \mathrm{NMR}\left(101 \mathrm{MHz}, \mathrm{CDCl}_{3}\right) \delta$ $143.69,136.93,134.35,133.48,133.11,129.87,128.92,128.23,127.90,127.56$, 127.29, 127.25, 126.77, 124.63, 68.77, 51.16, 43.93, 32.19, 31.26, 29.38, 28.37, 22.51, 21.64, 14.06. HR-ESI-MS $\mathrm{m} / z$ calcd. for $\mathrm{C}_{26} \mathrm{H}_{31} \mathrm{~N}_{4} \mathrm{O}_{2} \mathrm{~S}_{2}[\mathrm{M}-\mathrm{H}]^{-}: 495.1894$, found: 495.1894. HPLC (Daicel Chiralpak OD-H column, $i$-PrOH/hexane $=4 / 96,1 \mathrm{~mL} / \mathrm{min}$, $223 \mathrm{~nm}$ ) $\mathrm{t}_{1}=22.5 \mathrm{~min}$ (minor), $\mathrm{t}_{2}=26.2 \mathrm{~min}$ (major).

$\mathrm{mAU}$
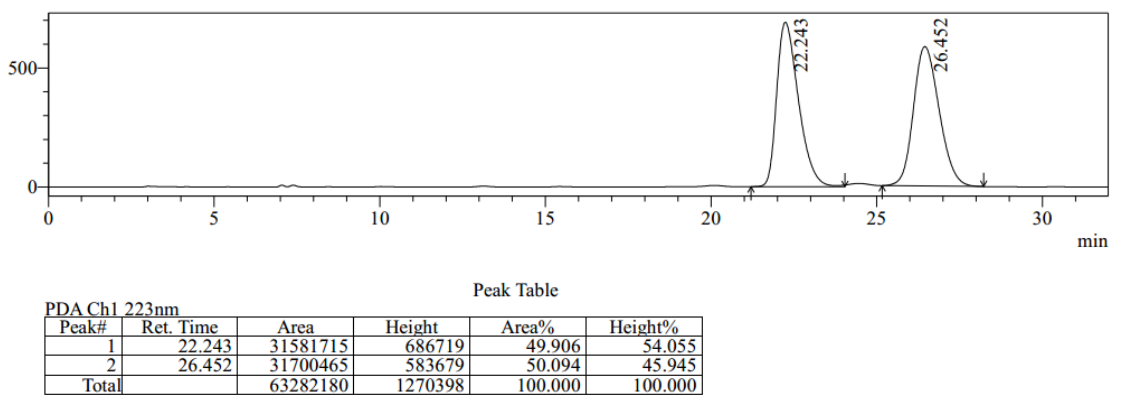


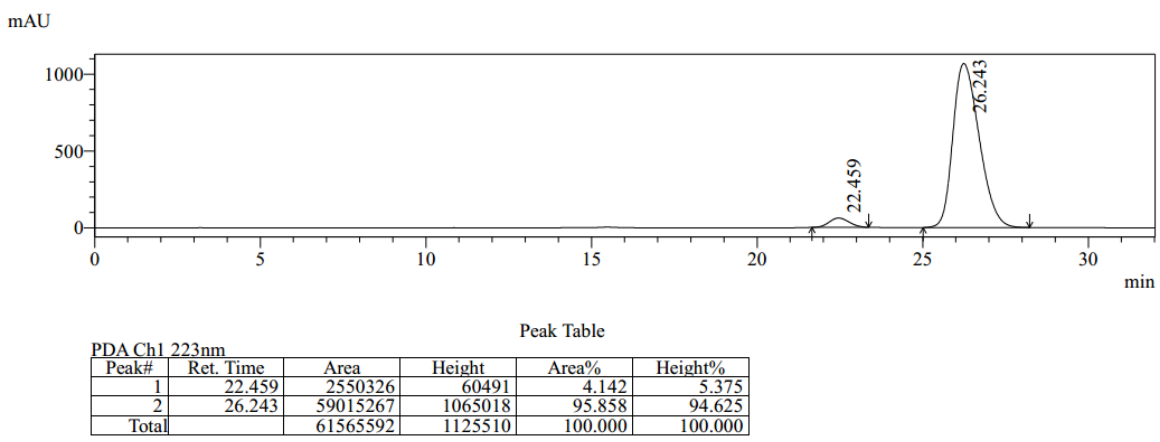

$\overbrace{\mathrm{I}}^{\mathrm{N}}$

$N$-((2S,3R)-3-Azido-2-(p-tolylthio)butyl)-4-methylbenzenesulfonamide (3z): Flash column chromatography (eluent: $\mathrm{PE} / \mathrm{EtOAc}=15 /$ to $5 / 1, \mathrm{v} / \mathrm{v}$ ) to afford $\mathbf{3 z}$ as a colorless oil. $35.5 \mathrm{mg}, 91 \%$ yield, $90 \%$ ee with $19: 1 \mathrm{dr} .[\alpha]^{25} \mathrm{D}=-27.9(\mathrm{c}=0.2$, $\left.\mathrm{CHCl}_{3}\right) .{ }^{1} \mathrm{H} \mathrm{NMR}\left(400 \mathrm{MHz}, \mathrm{CDCl}_{3}\right) \delta 7.72(\mathrm{~d}, J=8.3 \mathrm{~Hz}, 2 \mathrm{H}), 7.29(\mathrm{~d}, J=8.3 \mathrm{~Hz}$, 2H), $7.23-7.18(\mathrm{~m}, 2 \mathrm{H}), 7.06(\mathrm{~d}, J=7.9 \mathrm{~Hz}, 2 \mathrm{H}), 5.19(\mathrm{dd}, J=7.4,4.9 \mathrm{~Hz}, 1 \mathrm{H}), 3.63$ - $3.56(\mathrm{~m}, 1 \mathrm{H}), 3.28(\mathrm{ddd}, J=12.7,7.7,4.7 \mathrm{~Hz}, 1 \mathrm{H}), 3.03-2.84(\mathrm{~m}, 2 \mathrm{H}), 2.44(\mathrm{~s}$, $3 \mathrm{H}), 2.32(\mathrm{~s}, 3 \mathrm{H}), 1.39$ (d, $J=6.6 \mathrm{~Hz}, 3 \mathrm{H}) .{ }^{13} \mathrm{C} \mathrm{NMR}\left(101 \mathrm{MHz}, \mathrm{CDCl}_{3}\right) \delta 143.74$, 138.70, 136.80, 133.57, 130.24, 129.92, 128.48, 127.24, 58.85, 55.35, 43.37, 21.68, 21.26, 17.46. HR-ESI-MS $\mathrm{m} / z$ calcd. for $\mathrm{C}_{18} \mathrm{H}_{21} \mathrm{~N}_{4} \mathrm{O}_{2} \mathrm{~S}_{2}$ [M-H] : 389.1111, found: 389.1117. HPLC (Daicel Chiralpak OD-H column, $i$-PrOH/hexane $=2 / 97,1 \mathrm{~mL} / \mathrm{min}$, $223 \mathrm{~nm}$ ) $\mathrm{t}_{1}=32.4 \min$ (minor), $\mathrm{t}_{2}=36.8 \min$ (major).

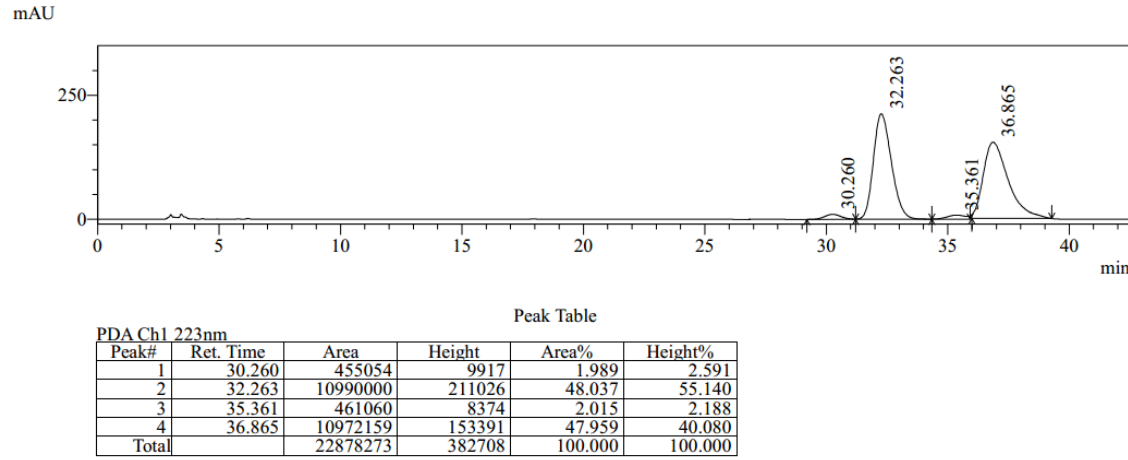



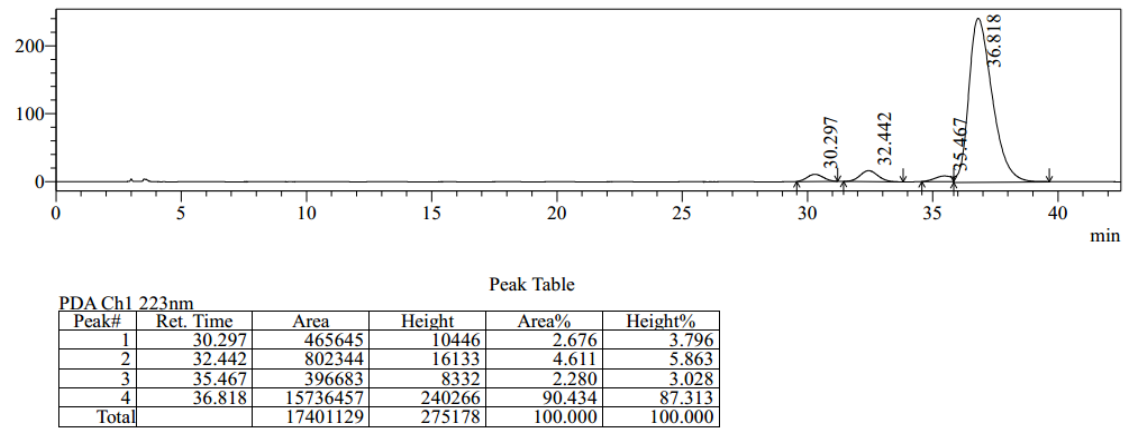<smiles>CCC[C@H](N)[C@H](CN[13CH3])Sc1ccc(C)cc1</smiles>

$N$-((2S,3R)-3-Azido-2-( $p$-tolylthio)hexyl)-4-methylbenzenesulfonamide

(3aa):

Flash column chromatography (eluent: PE/EtOAc $=15 / 1$ to $5 / 1, \mathrm{v} / \mathrm{v}$ ) to afford 3aa as a colorless oil. $34.3 \mathrm{mg}, 82 \%$ yield, $89 \%$ ee with $>99: 1 d r .[\alpha]^{25} \mathrm{D}=-12.5(\mathrm{c}=0.2$, $\left.\mathrm{CHCl}_{3}\right) .{ }^{1} \mathrm{H} \mathrm{NMR}\left(400 \mathrm{MHz}, \mathrm{CDCl}_{3}\right) \delta 7.72(\mathrm{~d}, J=8.2 \mathrm{~Hz}, 2 \mathrm{H}), 7.30(\mathrm{~d}, J=8.2 \mathrm{~Hz}$, $2 \mathrm{H}), 7.20(\mathrm{~d}, J=7.9 \mathrm{~Hz}, 2 \mathrm{H}), 7.07(\mathrm{~d}, J=7.9 \mathrm{~Hz}, 2 \mathrm{H}), 5.19(\mathrm{dd}, J=7.7,3.9 \mathrm{~Hz}, 1 \mathrm{H})$, $3.44(\mathrm{dt}, J=9.2,4.7 \mathrm{~Hz}, 1 \mathrm{H}), 3.28(\mathrm{dt}, J=21.4,7.7 \mathrm{~Hz}, 1 \mathrm{H}), 3.07-2.94(\mathrm{~m}, 2 \mathrm{H})$, $2.44(\mathrm{~s}, 3 \mathrm{H}), 2.33(\mathrm{~s}, 3 \mathrm{H}), 1.74-1.62(\mathrm{~m}, 2 \mathrm{H}), 1.62-1.52(\mathrm{~m}, 1 \mathrm{H}), 1.52-1.39(\mathrm{~m}$, $1 \mathrm{H}), 1.37-1.24(\mathrm{~m}, 1 \mathrm{H}), 0.90(\mathrm{t}, J=7.3 \mathrm{~Hz}, 3 \mathrm{H}) .{ }^{13} \mathrm{C} \mathrm{NMR}\left(101 \mathrm{MHz}, \mathrm{CDCl}_{3}\right) \delta$ 143.71, 138.67, 136.78, 133.55, 130.23, 129.90, 128.56, 127.23, 64.15, 54.31, 43.06, 34.46, 21.65, 21.25, 19.49, 13.80. HR-ESI-MS m/z calcd. for $\mathrm{C}_{20} \mathrm{H}_{25} \mathrm{~N}_{4} \mathrm{O}_{2} \mathrm{~S}_{2}[\mathrm{M}-\mathrm{H}]^{-}$: 417.1424, found: 417.1419. HPLC (Daicel Chiralpak OJ-H column, $i-\mathrm{PrOH} / \mathrm{hexane}=$ 10/90, $1 \mathrm{~mL} / \mathrm{min}, 223 \mathrm{~nm}$ ) $\mathrm{t}_{1}=10.8 \mathrm{~min}$ (major), $\mathrm{t}_{2}=12.9 \mathrm{~min}$ (minor).

$\mathrm{mAU}$
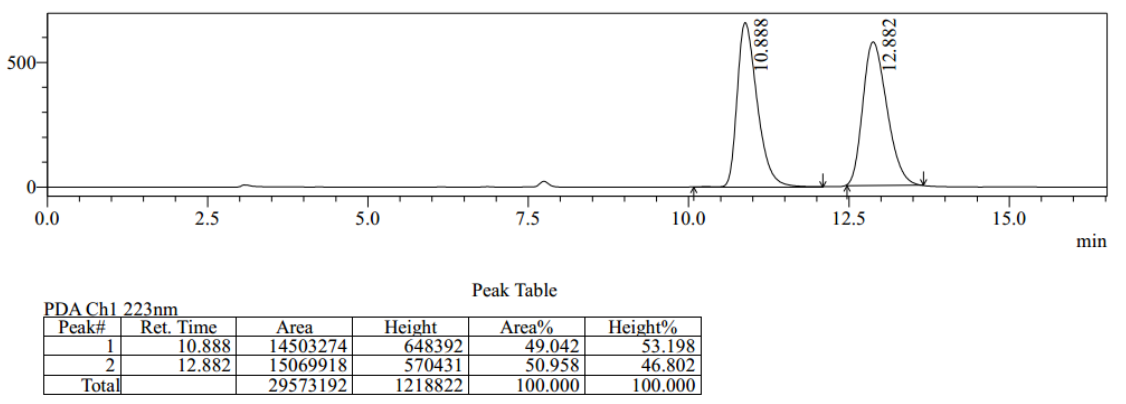
$\mathrm{mAU}$
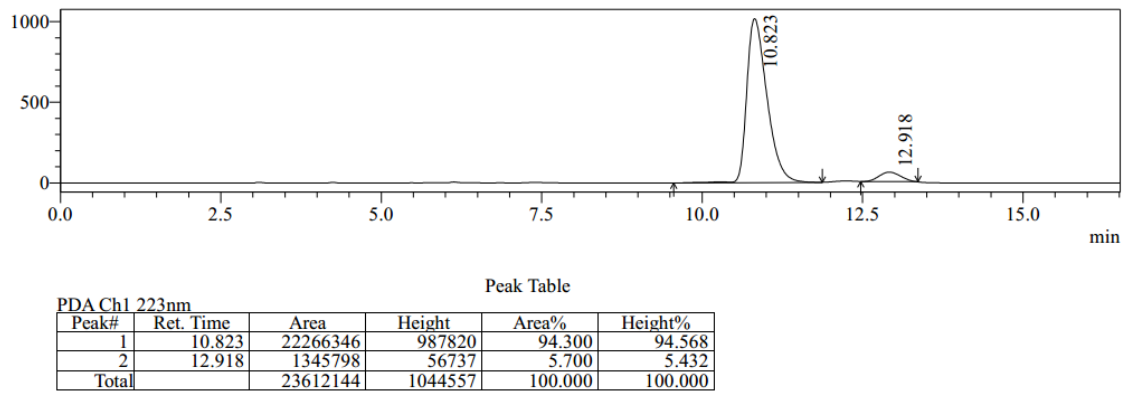<smiles>[CH2-]NC[C@@H](Sc1ccc(C)cc1)[C@H](N)CCc1ccccc1</smiles>

$N$-((2S,3R)-3-Azido-5-phenyl-2-(p-tolylthio)pentyl)-4-methylbenzenesulfonamide (3ab): Flash column chromatography (eluent: PE/EtOAc $=15 /$ to $5 / 1, \mathrm{v} / \mathrm{v}$ ) to afford 3ab as a colorless oil. $41.8 \mathrm{mg}, 87 \%$ yield, $91 \%$ ee with $21: 1 d r$. $[\alpha]^{25} \mathrm{D}=-14.4(\mathrm{c}=$ $\left.0.2, \mathrm{CHCl}_{3}\right) .{ }^{1} \mathrm{H} \mathrm{NMR}\left(400 \mathrm{MHz}, \mathrm{CDCl}_{3}\right) \delta 7.71(\mathrm{~d}, J=8.2 \mathrm{~Hz}, 2 \mathrm{H}), 7.29-7.17(\mathrm{~m}$, $6 \mathrm{H}), 7.14-7.09(\mathrm{~m}, 3 \mathrm{H}), 7.00(\mathrm{~d}, J=7.9 \mathrm{~Hz}, 2 \mathrm{H}), 5.22(\mathrm{dd}, J=7.6,4.3 \mathrm{~Hz}, 1 \mathrm{H}), 3.41$ (ddd, $J=9.3,5.6,3.8 \mathrm{~Hz}, 1 \mathrm{H}), 3.33-3.24(\mathrm{~m}, 1 \mathrm{H}), 3.05-2.91(\mathrm{~m}, 2 \mathrm{H}), 2.74(\mathrm{ddd}, J$ $=14.1,9.3,5.5 \mathrm{~Hz}, 1 \mathrm{H}), 2.65-2.55(\mathrm{~m}, 1 \mathrm{H}), 2.41(\mathrm{~s}, 3 \mathrm{H}), 2.31(\mathrm{~s}, 3 \mathrm{H}), 2.10-2.00$ (m, 1H), $1.94-1.81(\mathrm{~m}, 1 \mathrm{H}) .{ }^{13} \mathrm{C} \mathrm{NMR}\left(101 \mathrm{MHz}, \mathrm{CDCl}_{3}\right) \delta 143.76,140.48,138.78$, $136.79,133.73,130.26,129.94,128.72,128.56,128.08,127.24,126.38,63.24,54.31$, 43.07, 34.06, 32.24, 21.67, 21.26. HR-ESI-MS m/z calcd. for $\mathrm{C}_{25} \mathrm{H}_{27} \mathrm{~N}_{4} \mathrm{O}_{2} \mathrm{~S}_{2}[\mathrm{M}-\mathrm{H}]^{-}$: 479.1581, found: 479.1591. HPLC (Daicel Chiralpak IA column, $i$-PrOH/hexane $=$ $5 / 95,1 \mathrm{~mL} / \mathrm{min}, 223 \mathrm{~nm}$ ) $\mathrm{t}_{1}=19.9 \mathrm{~min}$ (minor), $\mathrm{t}_{2}=21.2 \mathrm{~min}$ (major).

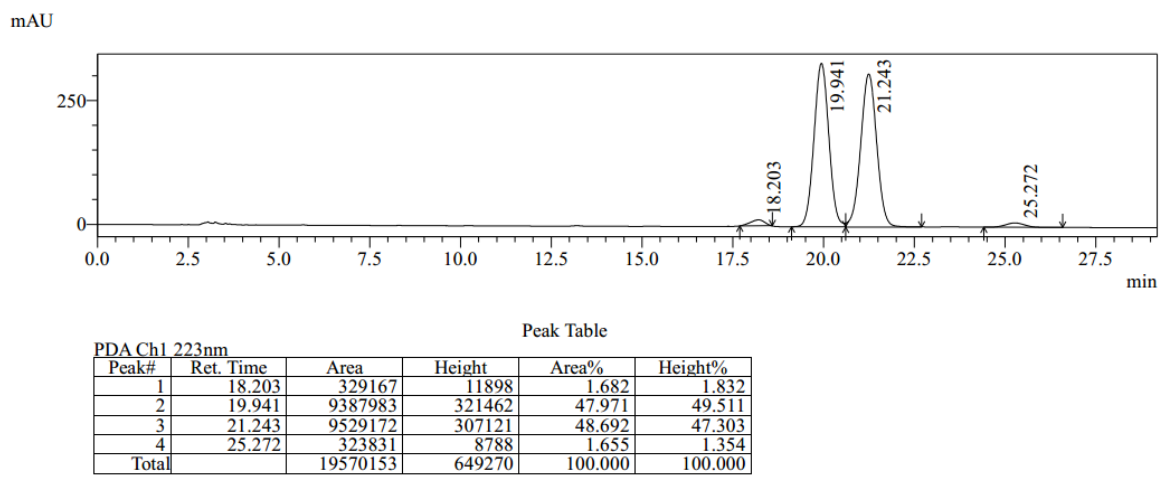


$\mathrm{mAU}$
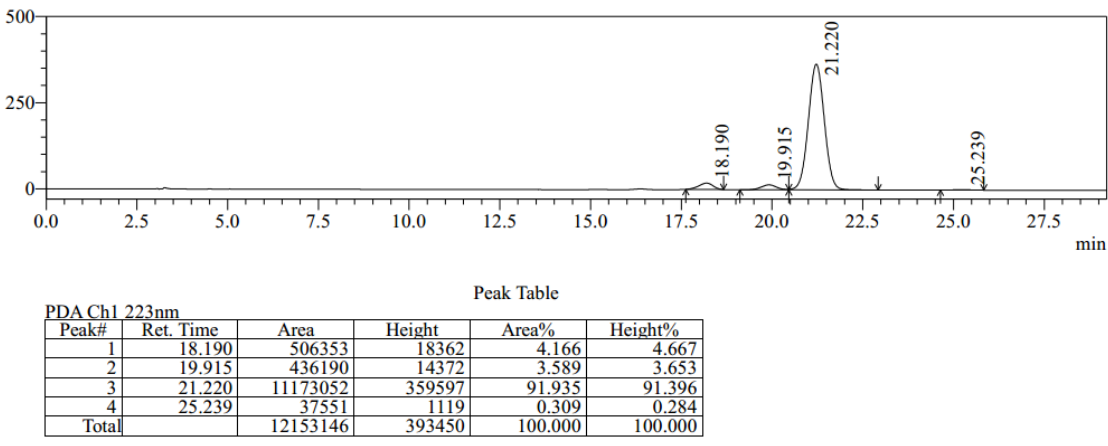

$\overbrace{\substack{\mathrm{s}^{n} \mathrm{Hex} \\ \mathrm{NHTs}}}^{\mathrm{OH}}$

$N$-((2S,3R)-2-(Hexylthio)-3-hydroxy-3-phenylpropyl)-4-methylbenzenesulfonami de (4a): Flash column chromatography (eluent: PE/EtOAc $=10 / 1$ to $3 / 1, \mathrm{v} / \mathrm{v}$ ) to afford $4 \mathbf{a}$ as a colorless oil. $29.9 \mathrm{mg}, 71 \%$ yield, $88 \%$ ee with $>99: 1 d r .[\alpha]^{25} \mathrm{D}=-26.4$ $\left(\mathrm{c}=0.2, \mathrm{CHCl}_{3}\right) .{ }^{1} \mathrm{H} \mathrm{NMR}\left(400 \mathrm{MHz}, \mathrm{CDCl}_{3}\right) \delta 7.67(\mathrm{~d}, J=8.2 \mathrm{~Hz}, 2 \mathrm{H}), 7.36-7.21$ $(\mathrm{m}, 7 \mathrm{H}), 5.19(\mathrm{t}, J=6.1 \mathrm{~Hz}, 1 \mathrm{H}), 4.71(\mathrm{~d}, J=6.5 \mathrm{~Hz}, 1 \mathrm{H}), 3.30-3.19(\mathrm{~m}, 1 \mathrm{H}), 3.12-$ $3.03(\mathrm{~m}, 1 \mathrm{H}), 2.86(\mathrm{dd}, J=11.7,6.1 \mathrm{~Hz}, 1 \mathrm{H}), 2.76(\mathrm{~s}, 1 \mathrm{H}), 2.43(\mathrm{~s}, 3 \mathrm{H}), 2.28-2.14$ $(\mathrm{m}, 2 \mathrm{H}), 1.42-1.31(\mathrm{~m}, 2 \mathrm{H}), 1.29-1.11(\mathrm{~m}, 7 \mathrm{H}), 0.87(\mathrm{t}, J=7.1 \mathrm{~Hz}, 3 \mathrm{H}) .{ }^{13} \mathrm{C}$ NMR $\left(101 \mathrm{MHz}, \mathrm{CDCl}_{3}\right) \delta 143.55,141.04,136.96,129.84,128.56,128.21,127.26,126.51$, 75.36, 52.72, 43.59, 31.93, 31.39, 29.54, 28.50, 22.61, 21.66, 14.14. HR-ESI-MS m/z calcd. for $\mathrm{C}_{22} \mathrm{H}_{31} \mathrm{NNaO}_{3} \mathrm{~S}_{2}[\mathrm{M}+\mathrm{Na}]^{+}$: 444.1638, found: 444.1646. HPLC (Daicel Chiralpak IA column, $i$-PrOH$/$ hexane $=10 / 90,1 \mathrm{~mL} / \mathrm{min}, 223 \mathrm{~nm}) \mathrm{t}_{1}=17.7 \mathrm{~min}$ (major), $\mathrm{t}_{2}=21.7 \mathrm{~min}$ (minor).

$\mathrm{mAU}$
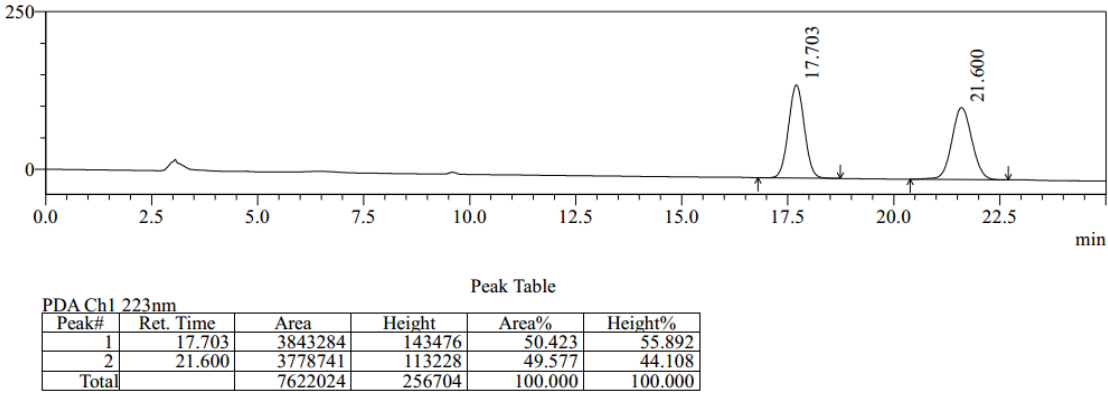

$\mathrm{mAU}$

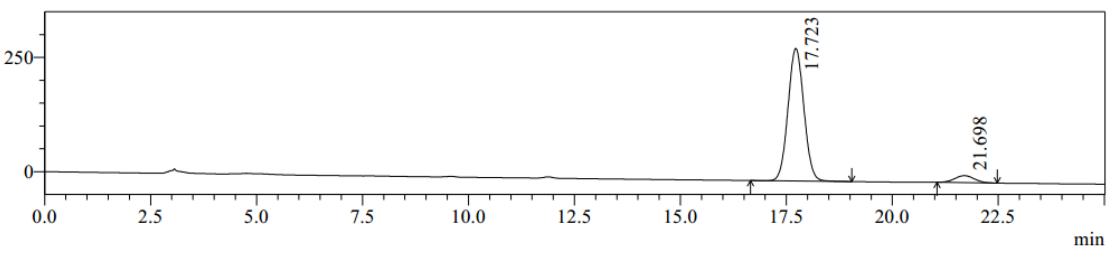

Peak Table

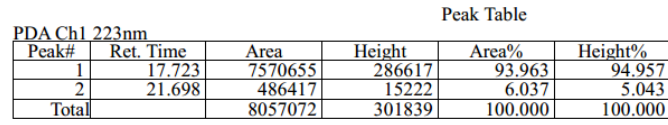


$\overbrace{\overline{\underline{\underline{S}}}^{n} \mathrm{Hex}}^{\mathrm{OEt}} \mathrm{NHTS}$

$N$-((2S,3R)-3-Ethoxy-2-(hexylthio)-3-phenylpropyl)-4-methylbenzenesulfonamide (4b): Flash column chromatography (eluent: PE/EtOAc $=15 / 1$ to $5 / 1, \mathrm{v} / \mathrm{v}$ ) to afford 4b as a colorless oil. $35.5 \mathrm{mg}, 79 \%$ yield, $91 \%$ ee with $>99: 1 d r .[\alpha]^{25} \mathrm{D}=-39.1(\mathrm{c}=$ 0.2, $\left.\mathrm{CHCl}_{3}\right) .{ }^{1} \mathrm{H} \mathrm{NMR}\left(400 \mathrm{MHz}, \mathrm{CDCl}_{3}\right) \delta 7.86-7.66(\mathrm{~m}, 2 \mathrm{H}), 7.33-7.26(\mathrm{~m}, 5 \mathrm{H})$, $7.22-7.16(\mathrm{~m}, 2 \mathrm{H}), 5.45(\mathrm{t}, J=6.0 \mathrm{~Hz}, 1 \mathrm{H}), 4.15(\mathrm{~d}, J=8.1 \mathrm{~Hz}, 1 \mathrm{H}), 3.38-3.26(\mathrm{~m}$, $2 \mathrm{H}), 3.26-3.12(\mathrm{~m}, 2 \mathrm{H}), 2.69(\mathrm{dt}, J=8.1,5.9 \mathrm{~Hz}, 1 \mathrm{H}), 2.44(\mathrm{~s}, 3 \mathrm{H}), 2.12-1.96(\mathrm{~m}$, $2 \mathrm{H}), 1.29-1.17(\mathrm{~m}, 4 \mathrm{H}), 1.16-1.09(\mathrm{~m}, 7 \mathrm{H}), 0.85(\mathrm{t}, J=7.2 \mathrm{~Hz}, 3 \mathrm{H}) .{ }^{13} \mathrm{C} \mathrm{NMR}$ $\left(101 \mathrm{MHz}, \mathrm{CDCl}_{3}\right) \delta 143.37,139.87,137.27,129.78,128.32,128.18,127.47,127.30$, 84.67, 64.88, 51.16, 44.72, 31.80, 31.36, 29.34, 28.41, 22.58, 21.64, 15.27, 14.12. HR-ESI-MS $\mathrm{m} / z$ calcd. for $\mathrm{C}_{24} \mathrm{H}_{35} \mathrm{NNaO}_{3} \mathrm{~S}_{2}[\mathrm{M}+\mathrm{Na}]^{+}: 472.1951$, found: 471.1942 . HPLC (Daicel Chiralpak OD-H column, $i$-PrOH/hexane = 3/97, $1 \mathrm{~mL} / \mathrm{min}, 231 \mathrm{~nm}$ ) $\mathrm{t}_{1}$ $=8.9 \underset{\operatorname{maU}}{\min }$ (minor), $\mathrm{t}_{2}=10.0 \min$ (major).
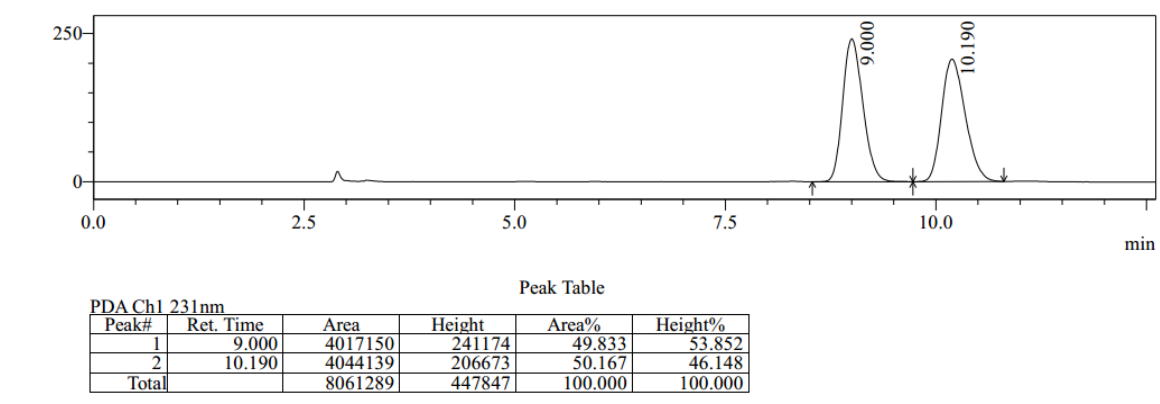

mAU
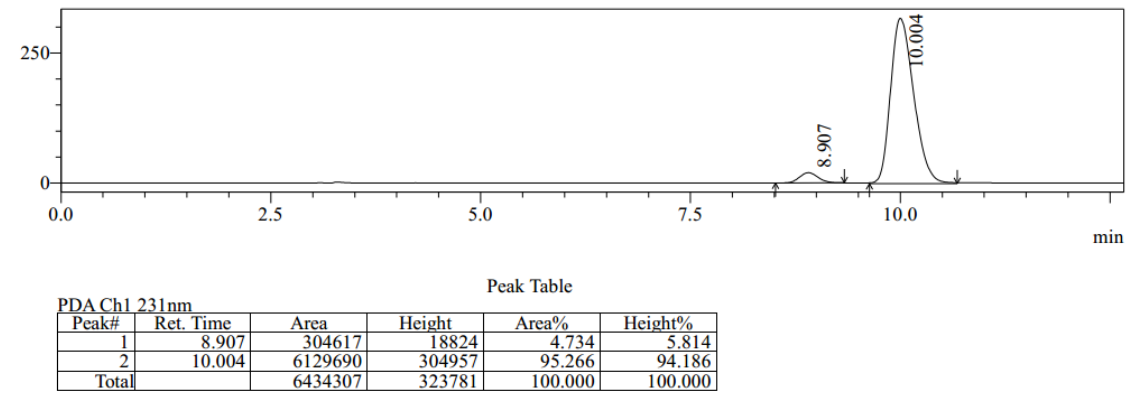

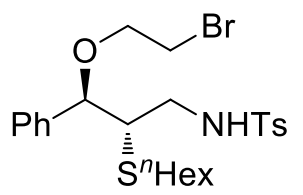

$N$-((2S,3R)-3-(2-Bromoethoxy)-2-(hexylthio)-3-phenylpropyl)-4-methylbenzenesu Ifonamide (4c): Flash column chromatography (eluent: $\mathrm{PE} / \mathrm{EtOAc}=15 / 1$ to $5 / 1$, v/v) to afford $\mathbf{4 c}$ as a colorless oil. $38.0 \mathrm{mg}, 72 \%$ yield, $93 \%$ ee with $>99: 1 d r$. $[\alpha]^{25} \mathrm{D}=$ -50.0 (c = 0.2, $\left.\mathrm{CHCl}_{3}\right) .{ }^{1} \mathrm{H} \mathrm{NMR}\left(400 \mathrm{MHz}, \mathrm{CDCl}_{3}\right) \delta 7.77(\mathrm{~d}, J=8.2 \mathrm{~Hz}, 2 \mathrm{H}), 7.38-$ $7.27(\mathrm{~m}, 5 \mathrm{H}), 7.27-7.20(\mathrm{~m}, 2 \mathrm{H}), 5.45(\mathrm{t}, J=6.3 \mathrm{~Hz}, 1 \mathrm{H}), 4.18(\mathrm{~d}, J=8.6 \mathrm{~Hz}, 1 \mathrm{H})$, $3.58(\mathrm{dt}, J=10.8,5.5 \mathrm{~Hz}, 1 \mathrm{H}), 3.48(\mathrm{dd}, J=11.3,5.5 \mathrm{~Hz}, 1 \mathrm{H}), 3.44-3.35(\mathrm{~m}, 3 \mathrm{H})$, $3.28-3.20(\mathrm{~m}, 1 \mathrm{H}), 2.76(\mathrm{dt}, J=8.6,5.9 \mathrm{~Hz}, 1 \mathrm{H}), 2.44(\mathrm{~s}, 3 \mathrm{H}), 2.10-1.94(\mathrm{~m}, 2 \mathrm{H})$, 
$1.30-1.17(\mathrm{~m}, 4 \mathrm{H}), 1.15-1.05(\mathrm{~m}, 4 \mathrm{H}), 0.85(\mathrm{t}, J=7.2 \mathrm{~Hz}, 3 \mathrm{H}) .{ }^{13} \mathrm{C}$ NMR $(101$ $\left.\mathrm{MHz}, \mathrm{CDCl}_{3}\right) \delta 143.39,138.98,137.32,129.78,128.56,128.46,127.68,127.34$, 85.10, 68.84, 51.37, 45.06, 31.94, 31.33, 31.02, 29.30, 28.36, 22.56, 21.65, 14.11. HR-ESI-MS $m / z$ calcd. for $\mathrm{C}_{24} \mathrm{H}_{34} \mathrm{NNaO}_{3} \mathrm{~S}_{2} \mathrm{Br}[\mathrm{M}+\mathrm{Na}]^{+}: 550.1056$, found: 550.1052 . HPLC (Daicel Chiralpak OD-H column, $i$-PrOH/hexane = 10/90, $1 \mathrm{~mL} / \mathrm{min}, 218 \mathrm{~nm}$ ) $\mathrm{t}_{1}=7.7 \min$ (minor), $\mathrm{t}_{2}=11.4$ min (major).

$\mathrm{mAU}$
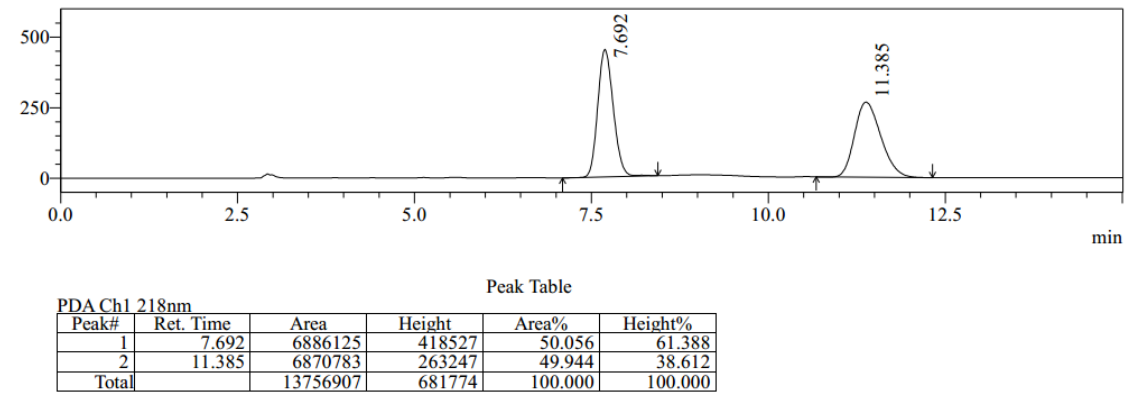

$\mathrm{mAU}$
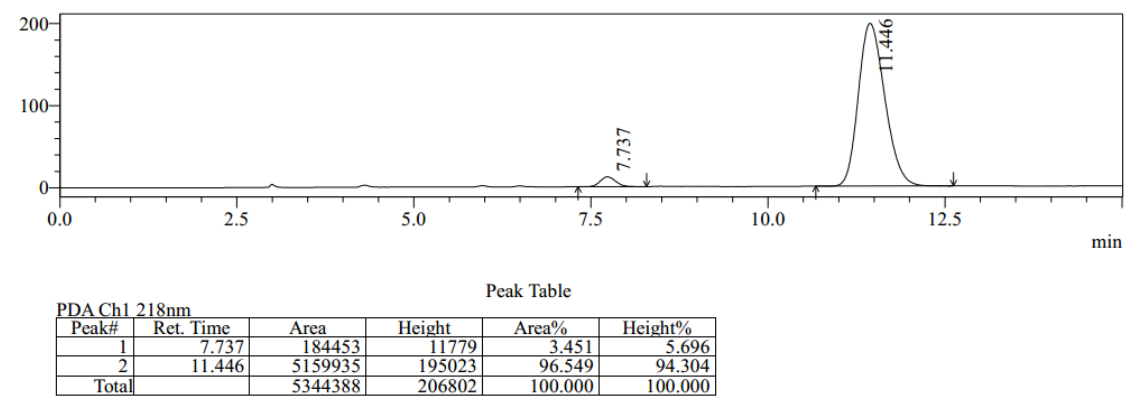

$\overbrace{\text { ì }}^{\mathrm{S}^{n} \mathrm{Hex}}$

$N$-((2S,3R)-2-(Hexylthio)-3-methoxy-3-phenylpropyl)-4-methylbenzenesulfonami de (4d): Flash column chromatography (eluent: PE/EtOAc $=15 / 1$ to $5 / 1, \mathrm{v} / \mathrm{v}$ ) to afford $4 \mathbf{d}$ as a colorless oil. $36.1 \mathrm{mg}, 83 \%$ yield, 94\% ee with $>99: 1 d r .[\alpha]^{25} \mathrm{D}=-40.2$ $\left(\mathrm{c}=0.2, \mathrm{CHCl}_{3}\right) .{ }^{1} \mathrm{H} \mathrm{NMR}\left(400 \mathrm{MHz}, \mathrm{CDCl}_{3}\right) \delta 7.75(\mathrm{~d}, J=8.1 \mathrm{~Hz}, 2 \mathrm{H}), 7.32-7.26$ $(\mathrm{m}, 5 \mathrm{H}), 7.20(\mathrm{~d}, J=7.4 \mathrm{~Hz}, 2 \mathrm{H}), 5.38(\mathrm{t}, J=5.8 \mathrm{~Hz}, 1 \mathrm{H}), 4.05(\mathrm{~d}, J=8.0 \mathrm{~Hz}, 1 \mathrm{H})$, $3.35(\mathrm{dt}, J=12.6,6.1 \mathrm{~Hz}, 1 \mathrm{H}), 3.18-3.06(\mathrm{~m}, 4 \mathrm{H}), 2.70(\mathrm{dd}, J=13.8,6.1 \mathrm{~Hz}, 1 \mathrm{H})$, $2.44(\mathrm{~s}, 3 \mathrm{H}), 2.17-1.85(\mathrm{~m}, 2 \mathrm{H}), 1.30-1.16(\mathrm{~m}, 4 \mathrm{H}), 1.16-1.07(\mathrm{~m}, 4 \mathrm{H}), 0.85(\mathrm{t}, J$ $=7.2 \mathrm{~Hz}, 3 \mathrm{H}) .{ }^{13} \mathrm{C} \mathrm{NMR}\left(101 \mathrm{MHz}, \mathrm{CDCl}_{3}\right) \delta 143.39,139.16,137.27,129.80,128.41$, $128.33,127.57,127.31,86.54,57.21,51.36,44.57,31.87,31.36,29.35,28.42,22.58$, 21.64, 14.13. HR-ESI-MS $m / z$ calcd. for $\mathrm{C}_{23} \mathrm{H}_{33} \mathrm{NNaO}_{3} \mathrm{~S}_{2}[\mathrm{M}+\mathrm{Na}]^{+}: 458.1794$, found: 458.1793. HPLC (Daicel Chiralpak OD-H column, $i$-PrOH/hexane $=1 / 99,1 \mathrm{~mL} / \mathrm{min}$, $223 \mathrm{~nm}$ ) $\mathrm{t}_{1}=20.5 \mathrm{~min}$ (minor), $\mathrm{t}_{2}=23.6 \mathrm{~min}$ (major). 
$\mathrm{mAU}$
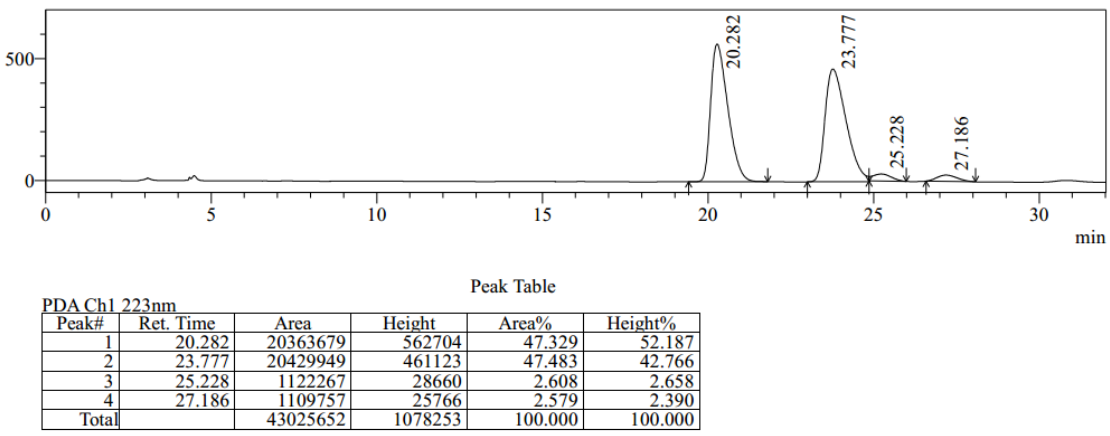

$\mathrm{mAU}$
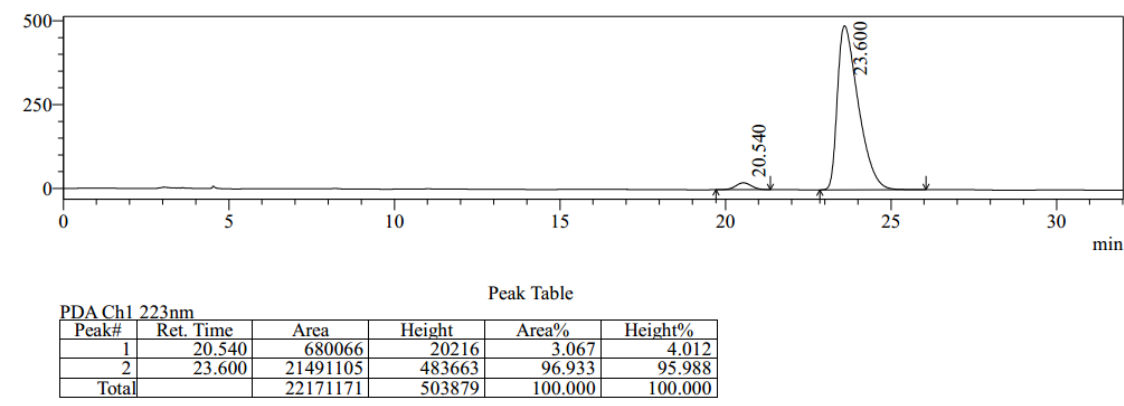

$\overbrace{\mathrm{s}_{S^{n} \mathrm{Hex}}}^{\mathrm{OBn}} \mathrm{NHTS}$

$N$-((2S,3R)-3-(Benzyloxy)-2-(hexylthio)-3-phenylpropyl)-4-methylbenzenesulfona mide (4e): Flash column chromatography (eluent: PE/EtOAc $=15 / 1$ to $5 / 1, \mathrm{v} / \mathrm{v}$ ) to afford $4 \mathbf{e}$ as a colorless oil. $42.4 \mathrm{mg}, 83 \%$ yield, $94 \%$ ee with $>99: 1 d r .[\alpha]^{25} \mathrm{D}=-78.9$ $\left(\mathrm{c}=0.2, \mathrm{CHCl}_{3}\right) .{ }^{1} \mathrm{H} \mathrm{NMR}\left(400 \mathrm{MHz}, \mathrm{CDCl}_{3}\right) \delta 7.68(\mathrm{~d}, J=8.2 \mathrm{~Hz}, 2 \mathrm{H}), 7.37-7.23$ $(\mathrm{m}, 10 \mathrm{H}), 7.22-7.18(\mathrm{~m}, 2 \mathrm{H}), 5.38(\mathrm{t}, J=6.1 \mathrm{~Hz}, 1 \mathrm{H}), 4.38(\mathrm{~d}, J=11.5 \mathrm{~Hz}, 1 \mathrm{H})$, $4.22(\mathrm{~d}, J=8.3 \mathrm{~Hz}, 1 \mathrm{H}), 4.15(\mathrm{~d}, J=11.5 \mathrm{~Hz}, 1 \mathrm{H}), 3.37(\mathrm{dt}, J=12.5,6.1 \mathrm{~Hz}, 1 \mathrm{H})$, $3.21-3.10(\mathrm{~m}, 1 \mathrm{H}), 2.76(\mathrm{dt}, J=8.3,6.0 \mathrm{~Hz}, 1 \mathrm{H}), 2.42(\mathrm{~s}, 3 \mathrm{H}), 2.07-1.91(\mathrm{~m}, 2 \mathrm{H})$, $1.28-1.15(\mathrm{~m}, 4 \mathrm{H}), 1.15-1.04(\mathrm{~m}, 4 \mathrm{H}), 0.84(\mathrm{t}, J=7.2 \mathrm{~Hz}, 3 \mathrm{H}) .{ }^{13} \mathrm{C}$ NMR $(101$ $\left.\mathrm{MHz}, \mathrm{CDCl}_{3}\right) \delta 143.35,139.24,137.65,137.16,129.77,128.56,128.46,128.42$, $127.98,127.95,127.79,127.28,83.67,70.90,51.40,44.80,31.88,31.32,29.29,28.37$, 22.56, 21.63, 14.11. HR-ESI-MS $m / z$ calcd. for $\mathrm{C}_{29} \mathrm{H}_{37} \mathrm{NNaO}_{3} \mathrm{~S}_{2}[\mathrm{M}+\mathrm{Na}]^{+}:$534.2107, found: 534.2121. HPLC (Daicel Chiralpak AD-H column, $i$-PrOH/hexane $=5 / 95,1$ $\mathrm{mL} / \mathrm{min}, 218 \mathrm{~nm}$ ) $\mathrm{t}_{1}=17.3 \min$ (minor), $\mathrm{t}_{2}=19.0 \min$ (major).

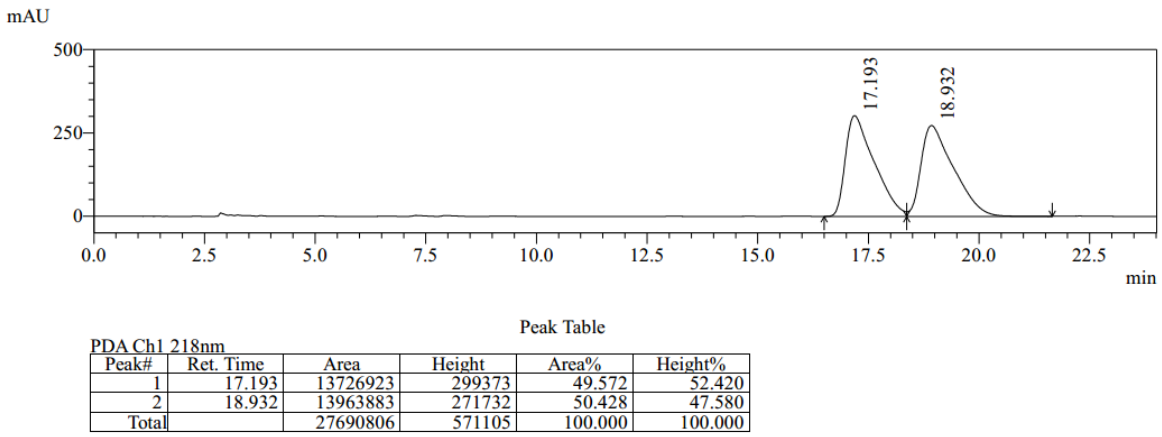



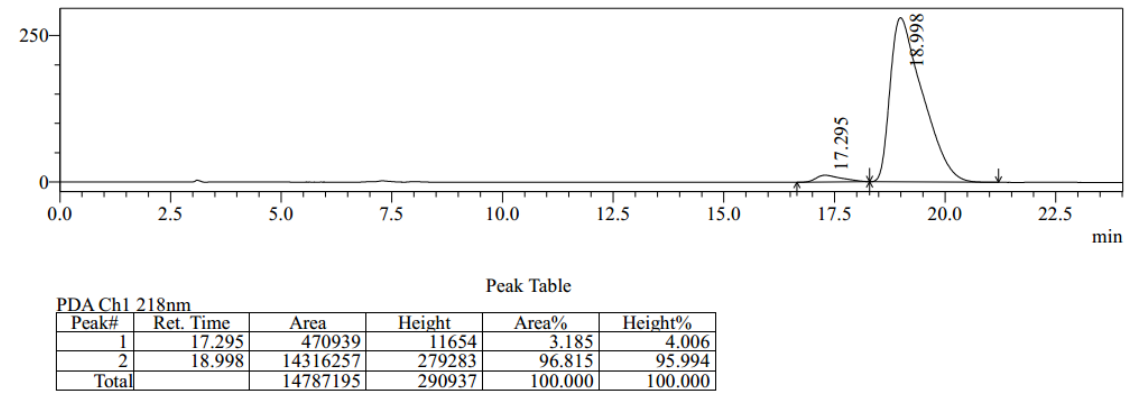

$\overbrace{\mathrm{i} \bar{s}^{n} \mathrm{Hex}}^{\mathrm{NHTs}}$

$N$-((2S,3R)-2-(Hexylthio)-3-phenyl-3-(prop-2-yn-1-yloxy)propyl)-4-methylbenzen esulfonamide (4f): Flash column chromatography (eluent: PE/EtOAc $=15 / 1$ to 5/1, $\mathrm{v} / \mathrm{v})$ to afford $4 \mathbf{f}$ as a colorless oil. $31.7 \mathrm{mg}, 69 \%$ yield, $92 \%$ ee with $>99: 1 d r$. $[\alpha]^{25} \mathrm{D}=$ -69.5 (c = 0.2, $\left.\mathrm{CHCl}_{3}\right) .{ }^{1} \mathrm{H} \mathrm{NMR}\left(400 \mathrm{MHz}, \mathrm{CDCl}_{3}\right) \delta 7.77(\mathrm{~d}, J=8.3 \mathrm{~Hz}, 2 \mathrm{H}), 7.34-$ $7.29(\mathrm{~m}, 5 \mathrm{H}), 7.27-7.20(\mathrm{~m}, 2 \mathrm{H}), 5.34(\mathrm{t}, J=6.2 \mathrm{~Hz}, 1 \mathrm{H}), 4.39(\mathrm{~d}, J=8.7 \mathrm{~Hz}, 1 \mathrm{H})$, $4.07(\mathrm{dd}, J=15.8,2.3 \mathrm{~Hz}, 1 \mathrm{H}), 3.75(\mathrm{dd}, J=15.8,2.3 \mathrm{~Hz}, 1 \mathrm{H}), 3.44$ (ddd, $J=12.9$, 6.7, $5.9 \mathrm{~Hz}, 1 \mathrm{H}), 3.17-3.06(\mathrm{~m}, 1 \mathrm{H}), 2.76(\mathrm{dt}, J=8.7,6.2 \mathrm{~Hz}, 1 \mathrm{H}), 2.44(\mathrm{~s}, 3 \mathrm{H}), 2.39$ $(\mathrm{t}, J=2.3 \mathrm{~Hz}, 1 \mathrm{H}), 2.08-1.91(\mathrm{~m}, 2 \mathrm{H}), 1.30-1.16(\mathrm{~m}, 4 \mathrm{H}), 1.15-1.05(\mathrm{~m}, 4 \mathrm{H})$, $0.85(\mathrm{t}, J=7.2 \mathrm{~Hz}, 3 \mathrm{H}) .{ }^{13} \mathrm{C} \mathrm{NMR}\left(101 \mathrm{MHz}, \mathrm{CDCl}_{3}\right) \delta 143.42,138.24,137.28$, $129.82,128.70,128.51,127.98,127.35,83.05,79.25,75.05,55.96,51.13,44.95$, $31.88,31.35,29.31,28.40,22.59,21.66,14.13$. HR-ESI-MS $\mathrm{m} / \mathrm{z}$ calcd. for $\mathrm{C}_{25} \mathrm{H}_{33} \mathrm{NNaO}_{3} \mathrm{~S}_{2}[\mathrm{M}+\mathrm{Na}]^{+}$: 482.1794, found: 482.1799. HPLC (Daicel Chiralpak OD-H column, $i$-PrOH/hexane $=3 / 97,1 \mathrm{~mL} / \mathrm{min}, 230 \mathrm{~nm}) \mathrm{t}_{1}=16.6 \mathrm{~min}($ minor $), \mathrm{t}_{2}=$ 18.9 min (major).

mAU

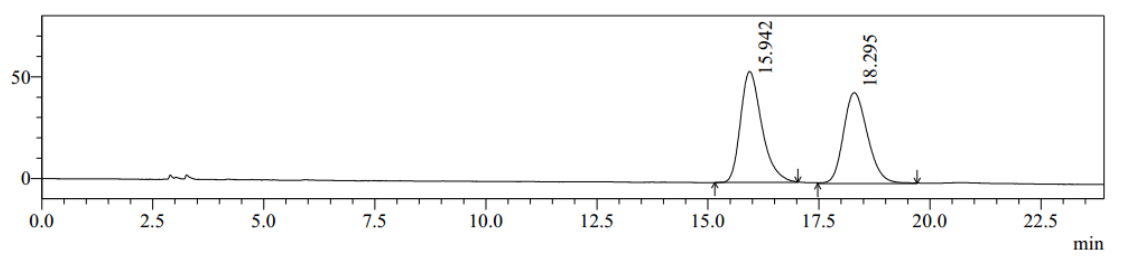

Peak Table

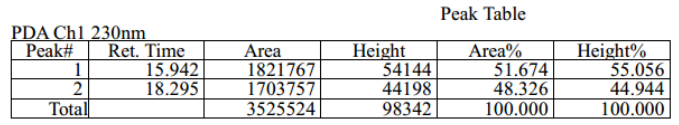


$\mathrm{mAU}$
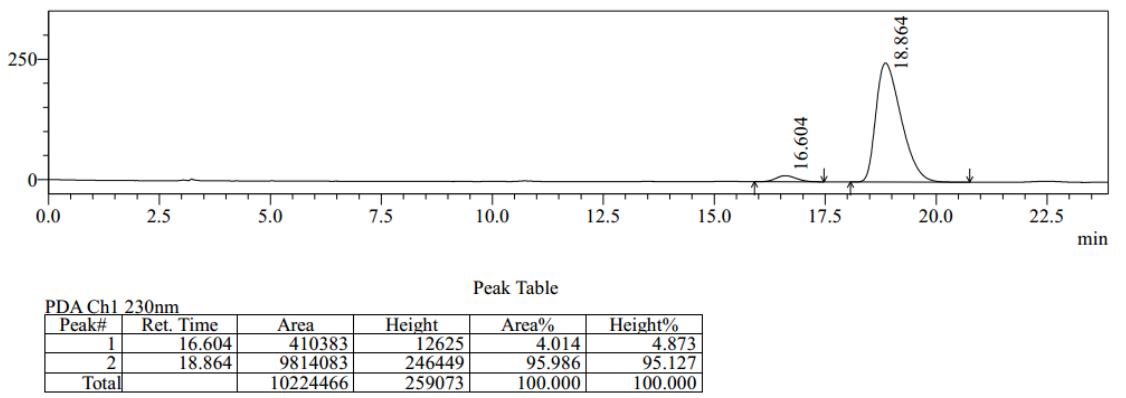

\section{Transformations of products}

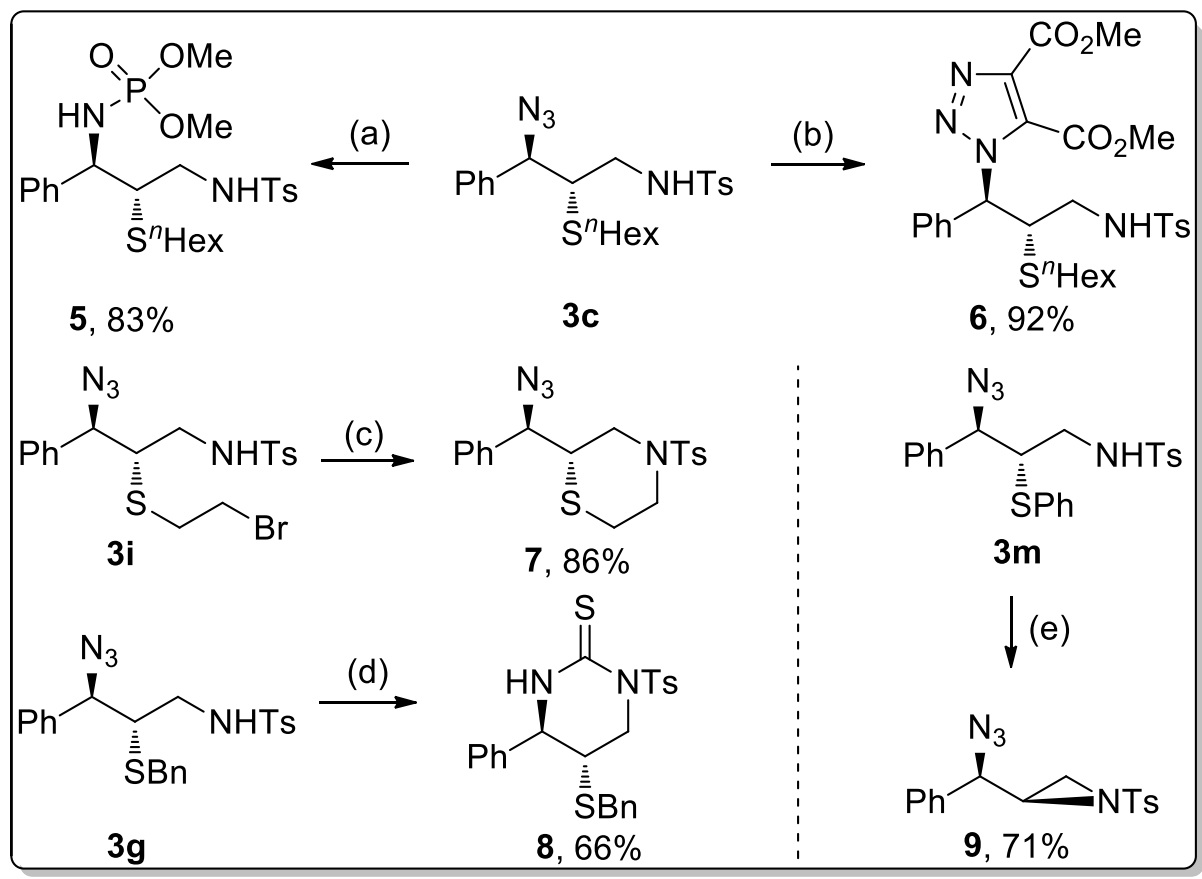

$\underset{\mathrm{Ph}_{\mathrm{\underline {S }}^{n} \mathrm{Hex}}}{\mathrm{NHTs}_{3}}+\mathrm{P}(\mathrm{OMe})_{3} \underset{80^{\circ} \mathrm{C}, 8 \mathrm{~h}}{\stackrel{\text { toluene }}{\longrightarrow}}$

$3 c$

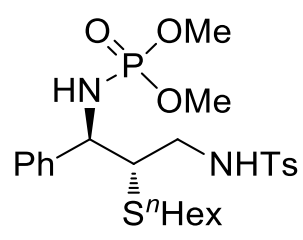

5

A solution of sulfide $3 \mathrm{c}(44.6 \mathrm{mg}, 0.1 \mathrm{mmol})$ and trimethyl phosphite $(14.2 \mu \mathrm{L}, 0.12$ mmol) in toluene $(2 \mathrm{~mL})$ was heated at $80{ }^{\circ} \mathrm{C}$ for $8 \mathrm{~h}$, and then cooled to room temperature and concentrated under reduced pressure. The residue was purified by flash silica gel column chromatography to yield the corresponding product. 


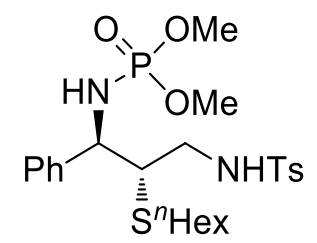

Dimethyl((1R,2S)-2-(hexylthio)-3-(4-methylphenylsulfonamido)-1-phenylpropyl) phosphoramidate (5): Flash column chromatography (eluent: $\mathrm{PE} / \mathrm{EtOAc}=3 / 1$ to $1 / 2$, $\mathrm{v} / \mathrm{v})$ to afford 5 as a white solid. $43.8 \mathrm{mg}, 83 \%$ yield and $94 \%$ ee. $\mathrm{mp} 106.8-108.0{ }^{\circ} \mathrm{C}$. $[\alpha]^{25} \mathrm{D}=2.2\left(\mathrm{c}=0.2, \mathrm{CHCl}_{3}\right) .{ }^{1} \mathrm{H} \mathrm{NMR}\left(400 \mathrm{MHz}, \mathrm{CDCl}_{3}\right) \delta 7.74(\mathrm{~d}, J=8.3 \mathrm{~Hz}, 1 \mathrm{H})$, $7.30-7.23(\mathrm{~m}, 7 \mathrm{H}), 5.75(\mathrm{dd}, J=8.0,4.4 \mathrm{~Hz}, 1 \mathrm{H}), 4.34(\mathrm{ddd}, J=10.9,9.7,6.3 \mathrm{~Hz}$, 1H), 4.26 (ddd, $J=10.9,9.7,6.3 \mathrm{~Hz}, 1 \mathrm{H}), 3.63(\mathrm{~d}, J=11.2 \mathrm{~Hz}, 3 \mathrm{H}), 3.38$ (d, $J=11.2$ $\mathrm{Hz}, 3 \mathrm{H}), 3.20-3.11(\mathrm{~m}, 1 \mathrm{H}), 3.01(\mathrm{dd}, J=13.3,6.3 \mathrm{~Hz}, 1 \mathrm{H}), 2.80$ (ddd, $J=13.3,7.3$, $4.4 \mathrm{~Hz}, 1 \mathrm{H}), \quad 2.42(\mathrm{~s}, 3 \mathrm{H}), 2.28-2.21(\mathrm{~m}, 2 \mathrm{H}), 1.46-1.35(\mathrm{~m}, 2 \mathrm{H}), 1.31-1.12(\mathrm{~m}$, $6 \mathrm{H}), 0.87(\mathrm{t}, J=7.0 \mathrm{~Hz}, 3 \mathrm{H}) .{ }^{13} \mathrm{C}$ NMR $\left(101 \mathrm{MHz}, \mathrm{CDCl}_{3}\right) \delta 143.45,140.17,136.77$, $129.73,128.30,127.82,127.25,127.14,77.40,53.41(\mathrm{dd}, J=6.5,3.6 \mathrm{~Hz}), 53.02(\mathrm{~d}, J$ $=5.2 \mathrm{~Hz}), 45.22,32.67,31.29,29.55,28.43,22.48,21.52,14.02 .{ }^{31} \mathrm{P}$ NMR $(162 \mathrm{MHz}$, $\left.\mathrm{CDCl}_{3}\right) \delta$ 9.54. HR-ESI-MS $m / z$ calcd. for $\mathrm{C}_{24} \mathrm{H}_{36} \mathrm{~N}_{2} \mathrm{O}_{5} \mathrm{PS}_{2}[\mathrm{M}-\mathrm{H}]^{-}: 527.1809$, found: 527.1820. HPLC (Daicel Chiralpak IA column, $i$-PrOH/hexane $=20 / 80,1 \mathrm{~mL} / \mathrm{min}$, $223 \mathrm{~nm}$ ) $\mathrm{t}_{1}=9.1 \mathrm{~min}$ (minor), $\mathrm{t}_{2}=11.3 \min$ (major).
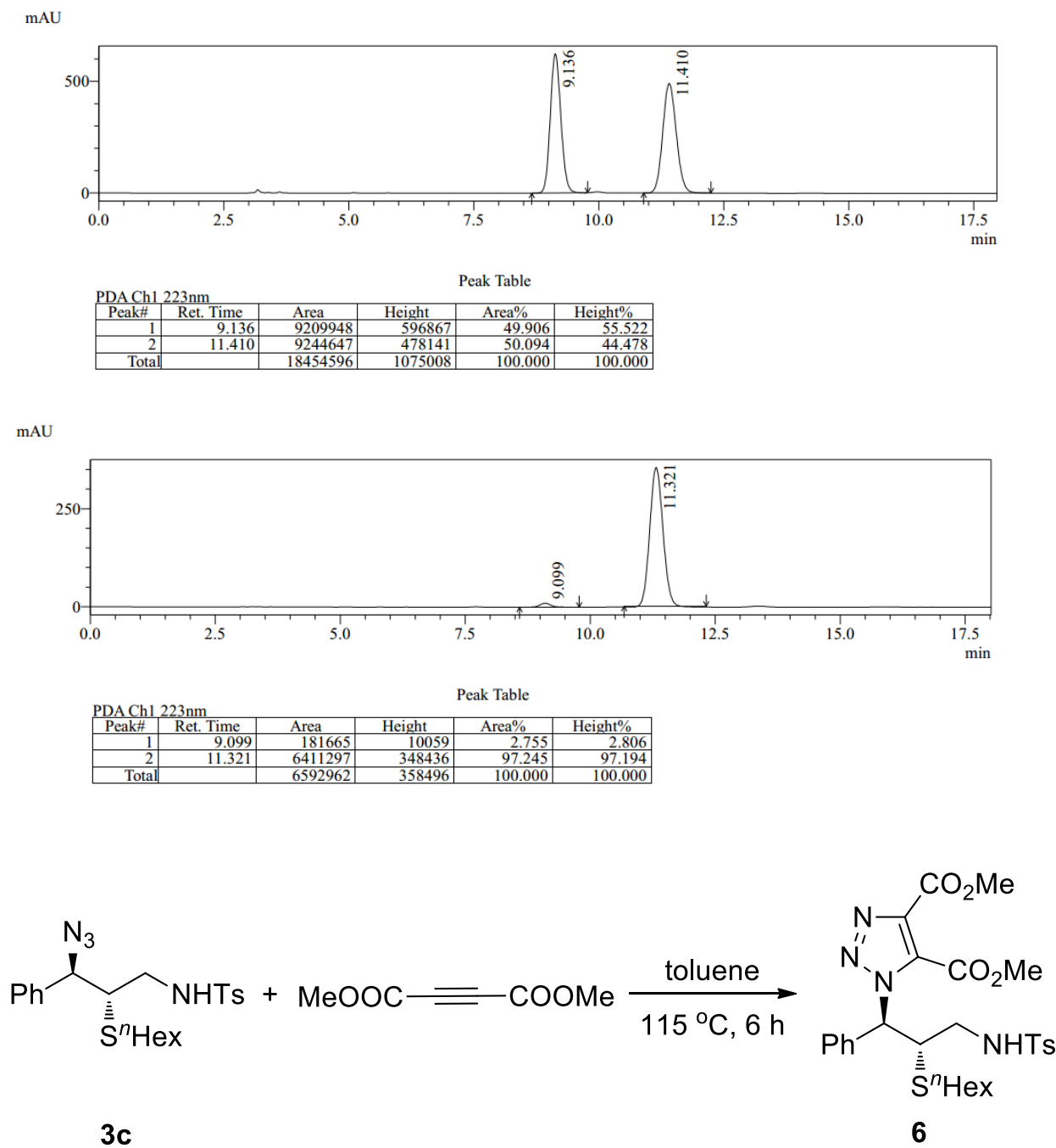
The reaction was carried out by modifying the literature procedure. ${ }^{17} \mathrm{~A}$ solution of sulfide $3 \mathrm{c}$ (44.6 mg, $0.1 \mathrm{mmol})$ and dimethyl but-2-ynedioate $(61.4 \mu \mathrm{L}, 0.11 \mathrm{mmol})$ in toluene $(2 \mathrm{~mL})$ was heated at $115{ }^{\circ} \mathrm{C}$ for $6 \mathrm{~h}$, and then cooled to room temperature and concentrated under reduced pressure. The residue was purified by flash silica gel column chromatography to yield the corresponding product.

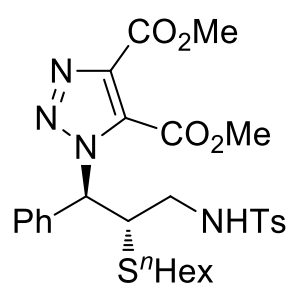

Dimethyl-1-((1R,2S)-2-(hexylthio)-3-(4-methylphenylsulfonamido)-1-phenylprop yl)-1H-1,2,3-triazole-4,5-dicarboxylate (6): Flash column chromatography (eluent: $\mathrm{PE} / \mathrm{EtOAc}=10 / 1$ to $2 / 1, \mathrm{v} / \mathrm{v})$ to afford 6 as a colorless oil. $54.1 \mathrm{mg}, 92 \%$ yield and $94 \%$ ee. $[\alpha]^{25} \mathrm{D}=44.0\left(\mathrm{c}=0.2, \mathrm{CHCl}_{3}\right) .{ }^{1} \mathrm{H} \mathrm{NMR}\left(400 \mathrm{MHz}, \mathrm{CDCl}_{3}\right) \delta 7.61(\mathrm{~d}, J=8.3$ $\mathrm{Hz}, 2 \mathrm{H}), 7.50-7.44(\mathrm{~m}, 2 \mathrm{H}), 7.36-7.30(\mathrm{~m}, 3 \mathrm{H}), 7.27(\mathrm{~d}, J=8.3 \mathrm{~Hz}, 2 \mathrm{H}), 6.17$ (d, $J$ $=10.8 \mathrm{~Hz}, 1 \mathrm{H}), 5.12(\mathrm{dd}, J=7.4,6.0 \mathrm{~Hz}, 1 \mathrm{H}), 4.12-3.70(\mathrm{~m}, 7 \mathrm{H}), 3.11(\mathrm{ddd}, J=$ $13.4,5.7,3.3 \mathrm{~Hz}, 1 \mathrm{H}), 2.96$ (ddd, $J=13.4,7.7,5.7 \mathrm{~Hz}, 1 \mathrm{H}), 2.41(\mathrm{~s}, 3 \mathrm{H}), 2.12-1.95$ $(\mathrm{m}, 2 \mathrm{H}), 1.33-1.16(\mathrm{~m}, 4 \mathrm{H}), 1.14-1.04(\mathrm{~m}, 4 \mathrm{H}), 0.84(\mathrm{t}, J=7.2 \mathrm{~Hz}, 3 \mathrm{H}) .{ }^{13} \mathrm{C} \mathrm{NMR}$ $\left(101 \mathrm{MHz}, \mathrm{CDCl}_{3}\right) \delta 160.56,158.81,143.70,140.06,136.50,136.15,130.57,129.85$, 129.10, 128.68, 128.54, 127.11, 65.36, 53.48, 52.81, 49.68, 43.93, 32.28, 31.20, 29.10, 28.23, 22.49, 21.58, 14.04. HR-ESI-MS $\mathrm{m} / \mathrm{z}$ calcd. for $\mathrm{C}_{28} \mathrm{H}_{35} \mathrm{~N}_{4} \mathrm{O}_{6} \mathrm{~S}_{2} \quad[\mathrm{M}-\mathrm{H}]^{-}$: 587.2003, found: 587.2001. HPLC (Daicel Chiralpak AD-H column, $i$-PrOH/hexane $=25 / 75,1 \mathrm{~mL} / \mathrm{min}, 223 \mathrm{~nm}$ ) $\mathrm{t}_{1}=13.0 \mathrm{~min}$ (major), $\mathrm{t}_{2}=17.0 \mathrm{~min}$ (minor).

$\mathrm{mAU}$
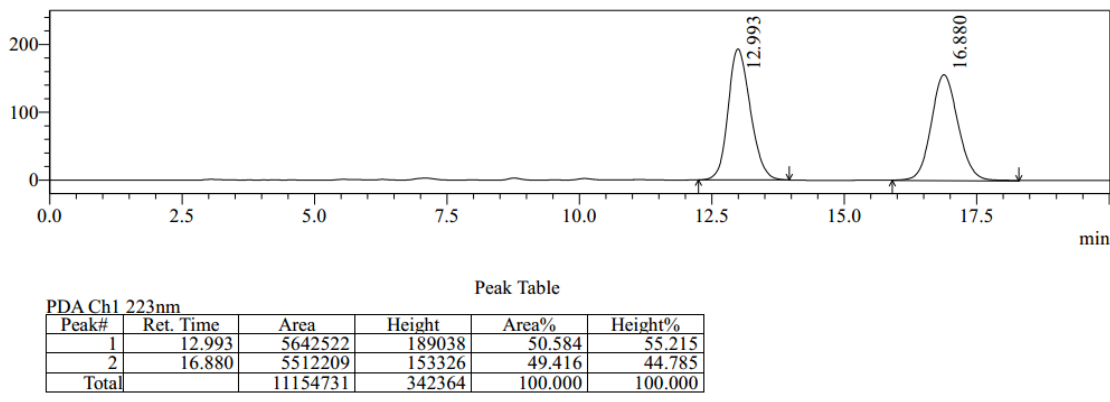

$\mathrm{mAU}$
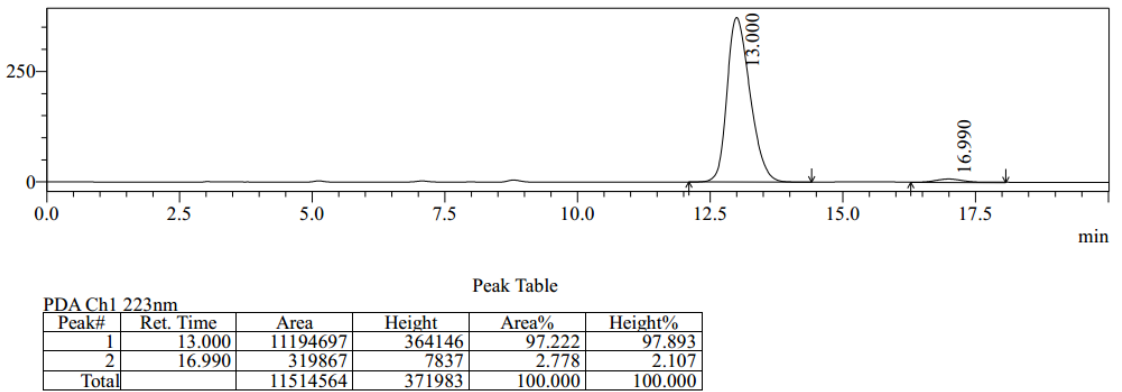
<smiles>NC(c1ccccc1)[C@H](CN[18OH])SCCBr</smiles>

3i<smiles>N[C@@H](c1[R17]ccc1)[C@H]1C[NH+](S)CCS1</smiles>

7

A suspension of sulfide $3 \mathbf{i}(46.8 \mathrm{mg}, 0.1 \mathrm{mmol})$ and $\mathrm{NaH}(60 \%, 4.4 \mathrm{mg}, 0.11 \mathrm{mmol})$ in DMF (2 mL) was stirred at room temperature for $8 \mathrm{~h}$. Then, the reaction was quenched with water $(2 \mathrm{~mL})$. The mixture was extracted with EtOAc $(3 \mathrm{~mL} \times 3)$ and washed with water $(10 \mathrm{~mL} \times 3)$. The organic layers were dried over $\mathrm{Na}_{2} \mathrm{SO}_{4}$, filtered, and concentrated under reduced pressure. The residue was purified by flash silica gel collumn chromotography to give the corresponding product.<smiles>NC(c1ccccc1)[C@H]1C[NH2+]CCS1</smiles>

(S)-2-((R)-Azido(phenyl)methyl)-4-tosylthiomorpholine (7): Flash column chromatography (eluent: PE/EtOAc $=15 / 1$ to $5 / 1$, v/v) to afford 7 as a white solid. $33.4 \mathrm{mg}, 86 \%$ yield and $94 \%$ ee. $\mathrm{mp} 96.3-98.2{ }^{\circ} \mathrm{C} .[\alpha]^{25} \mathrm{D}=-127.0\left(\mathrm{c}=0.2, \mathrm{CHCl}_{3}\right)$. ${ }^{1} \mathrm{H}$ NMR $\left(400 \mathrm{MHz}, \mathrm{CDCl}_{3}\right) \delta 7.65(\mathrm{~d}, J=8.2 \mathrm{~Hz}, 2 \mathrm{H}), 7.44-7.37(\mathrm{~m}, 3 \mathrm{H}), 7.34(\mathrm{~d}$, $J=8.2 \mathrm{~Hz}, 2 \mathrm{H}), 7.31-7.27(\mathrm{~m}, 2 \mathrm{H}), 4.53(\mathrm{~d}, J=9.0 \mathrm{~Hz}, 1 \mathrm{H}), 3.85(\mathrm{~d}, J=10.4 \mathrm{~Hz}$, $1 \mathrm{H}), 3.57(\mathrm{dt}, J=9.0,4.2 \mathrm{~Hz}, 1 \mathrm{H}), 3.16-3.00(\mathrm{~m}, 3 \mathrm{H}), 2.71-2.61(\mathrm{~m}, 2 \mathrm{H}), 2.44(\mathrm{~s}$, $3 \mathrm{H}) .{ }^{13} \mathrm{C} \mathrm{NMR}\left(101 \mathrm{MHz}, \mathrm{CDCl}_{3}\right) \delta 143.97,136.50,133.96,129.96,129.20,128.97$, 127.66, 127.50, 66.60, 50.12, 47.39, 44.04, 26.50, 21.62. HR-ESI-MS $\mathrm{m} / \mathrm{z}$ calcd. for $\mathrm{C}_{18} \mathrm{H}_{20} \mathrm{~N}_{4} \mathrm{NaO}_{2} \mathrm{~S}_{2}[\mathrm{M}+\mathrm{Na}]^{+}:$411.0920, found: 411.0928. HPLC (Daicel Chiralpak OJ-H column, $i-\mathrm{PrOH} /$ hexane $=25 / 75,1 \mathrm{~mL} / \mathrm{min}, 223 \mathrm{~nm}) \mathrm{t}_{1}=23.4 \mathrm{~min}($ minor $), \mathrm{t}_{2}=$ 28.5 min (major).

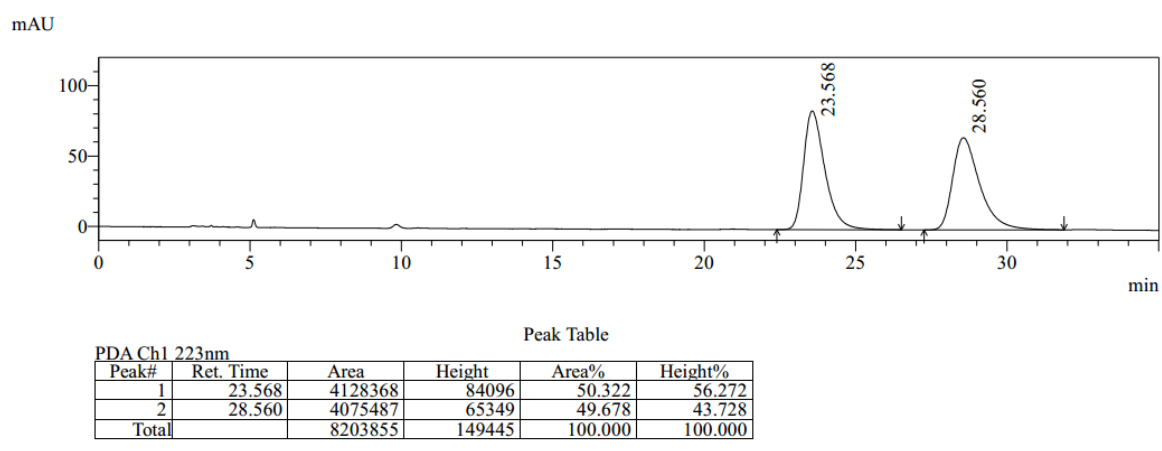



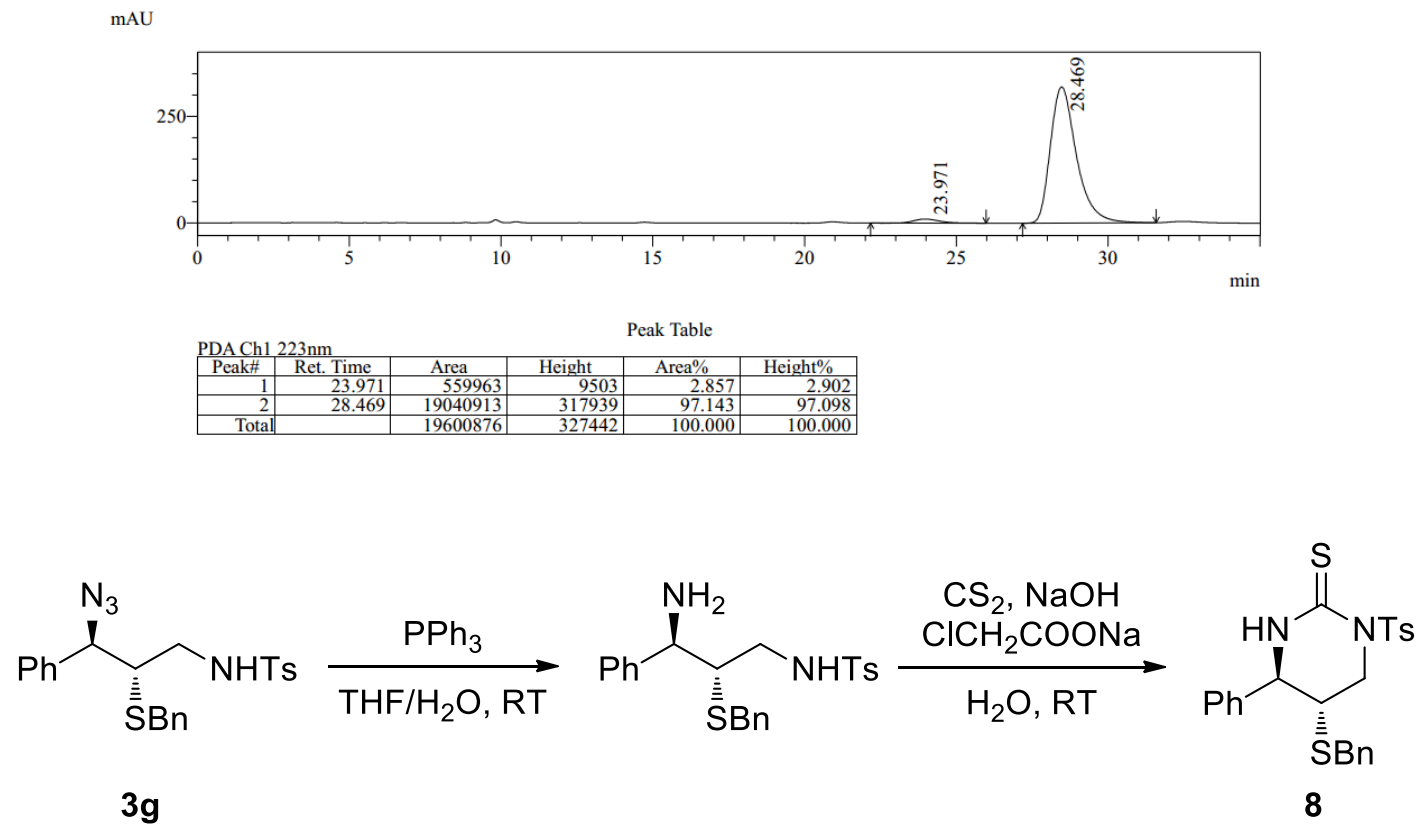

To a solution of sulfide $3 \mathrm{~g}(90.4 \mathrm{mg}, 0.2 \mathrm{mmol})$ in $\mathrm{THF} / \mathrm{H}_{2} \mathrm{O}(2 \mathrm{~mL} / 0.5 \mathrm{~mL})$ was added $\mathrm{PPh}_{3}(62.9 \mathrm{mg}, 0.24 \mathrm{mmol})$. The solution was stirred at room temperature overnight and then concentrated under reduced pressure. The residue was purified by flash silica gel collumn chromotography (eluent: $\mathrm{PE} / \mathrm{EtOAc}=1 / 1$ to EtOAc, v/v) to give the amine product as a colorless oil ( $81.8 \mathrm{mg}, 96 \%$ yield).

To a solution of amine $(81.8 \mathrm{mg}, 0.19 \mathrm{mmol})$ in $\mathrm{H}_{2} \mathrm{O}(2 \mathrm{~mL})$ were added $\mathrm{ClCH}_{2} \mathrm{COONa}$ (44.3 mg, $\left.0.38 \mathrm{mmol}\right), \mathrm{NaOH}(15.2 \mathrm{mg}, 0.38 \mathrm{mmol})$ and $\mathrm{CS}_{2}(22.9 \mu \mathrm{L}$, $0.38 \mathrm{mmol}$ ) successively. The mixture was stirred at room temperature for $12 \mathrm{~h}$ and then extracted with EtOAc $(3 \mathrm{~mL} \times 3)$. The organic layers were dried over $\mathrm{Na}_{2} \mathrm{SO}_{4}$, filtered and concentrated under reduced pressure. The residue was purified by flash silica gel collumn chromotography (eluent: $\mathrm{PE} / \mathrm{EtOAc}=5 / 1$ to $2 / 1, \mathrm{v} / \mathrm{v}$ ) to give the corresponding product 8 as a white solid (123 mg, 69\% yield).<smiles>S=C1NC(c2ccccc2)C(Cc2ccccc2)C[NH2+]1</smiles>

(4R,5S)-5-(Benzylthio)-4-phenyl-1-tosyltetrahydropyrimidine-2(1H)-thione $\quad(8)$ : Flash column chromatography (eluent: PE/EtOAc $=5 / 1$ to $2 / 1$, v/v) to afford 8 as a white solid. $123 \mathrm{mg}, 66 \%$ yield (two steps) and $90 \%$ ee. $\mathrm{mp} 147.6-148.8{ }^{\circ} \mathrm{C}$. $[\alpha]^{25} \mathrm{D}=$ $2.3\left(\mathrm{c}=0.2, \mathrm{CHCl}_{3}\right) .{ }^{1} \mathrm{H} \mathrm{NMR}\left(400 \mathrm{MHz}, \mathrm{CDCl}_{3}\right) \delta 7.81(\mathrm{~d}, J=8.3 \mathrm{~Hz}, 2 \mathrm{H}), 7.37-$ $7.23(\mathrm{~m}, 8 \mathrm{H}), 7.22-7.17(\mathrm{~m}, 2 \mathrm{H}), 7.09(\mathrm{~s}, 1 \mathrm{H}), 7.08-7.02(\mathrm{~m}, 2 \mathrm{H}), 4.31(\mathrm{dd}, J=$ 13.2, $3.7 \mathrm{~Hz}, 1 \mathrm{H}), 4.25(\mathrm{dd}, J=7.5,1.6 \mathrm{~Hz}, 1 \mathrm{H}), 3.86(\mathrm{dd}, J=13.2,8.7 \mathrm{~Hz}, 1 \mathrm{H}), 3.68$ $(\mathrm{d}, J=13.8 \mathrm{~Hz}, 1 \mathrm{H}), 3.56(\mathrm{~d}, J=13.8 \mathrm{~Hz}, 1 \mathrm{H}), 2.92(\mathrm{td}, J=8.3,3.7 \mathrm{~Hz}, 1 \mathrm{H}), 2.42(\mathrm{~s}$, $3 \mathrm{H}) .{ }^{13} \mathrm{C} \mathrm{NMR}\left(101 \mathrm{MHz}, \mathrm{CDCl}_{3}\right) \delta 177.51,144.61,138.13,136.64,136.33,129.17$, 
129.10, 129.04, 128.99, 128.94, 128.86, 127.65, 126.94, 62.24, 48.56, 42.80, 35.86, 21.79. HR-ESI-MS $m / z$ calcd. for $\mathrm{C}_{24} \mathrm{H}_{25} \mathrm{~N}_{2} \mathrm{O}_{2} \mathrm{~S}_{3}[\mathrm{M}+\mathrm{H}]^{+}: 469.1073$, found: 469.1068 . HPLC (Daicel Chiralpak IA column, $i-\mathrm{PrOH} / \mathrm{h}$ exane $=15 / 85,1 \mathrm{~mL} / \mathrm{min}, 215 \mathrm{~nm}$ ) $\mathrm{t}_{1}=$ $12.3 \min$ (major), $\mathrm{t}_{2}=13.3 \mathrm{~min}$ (minor).
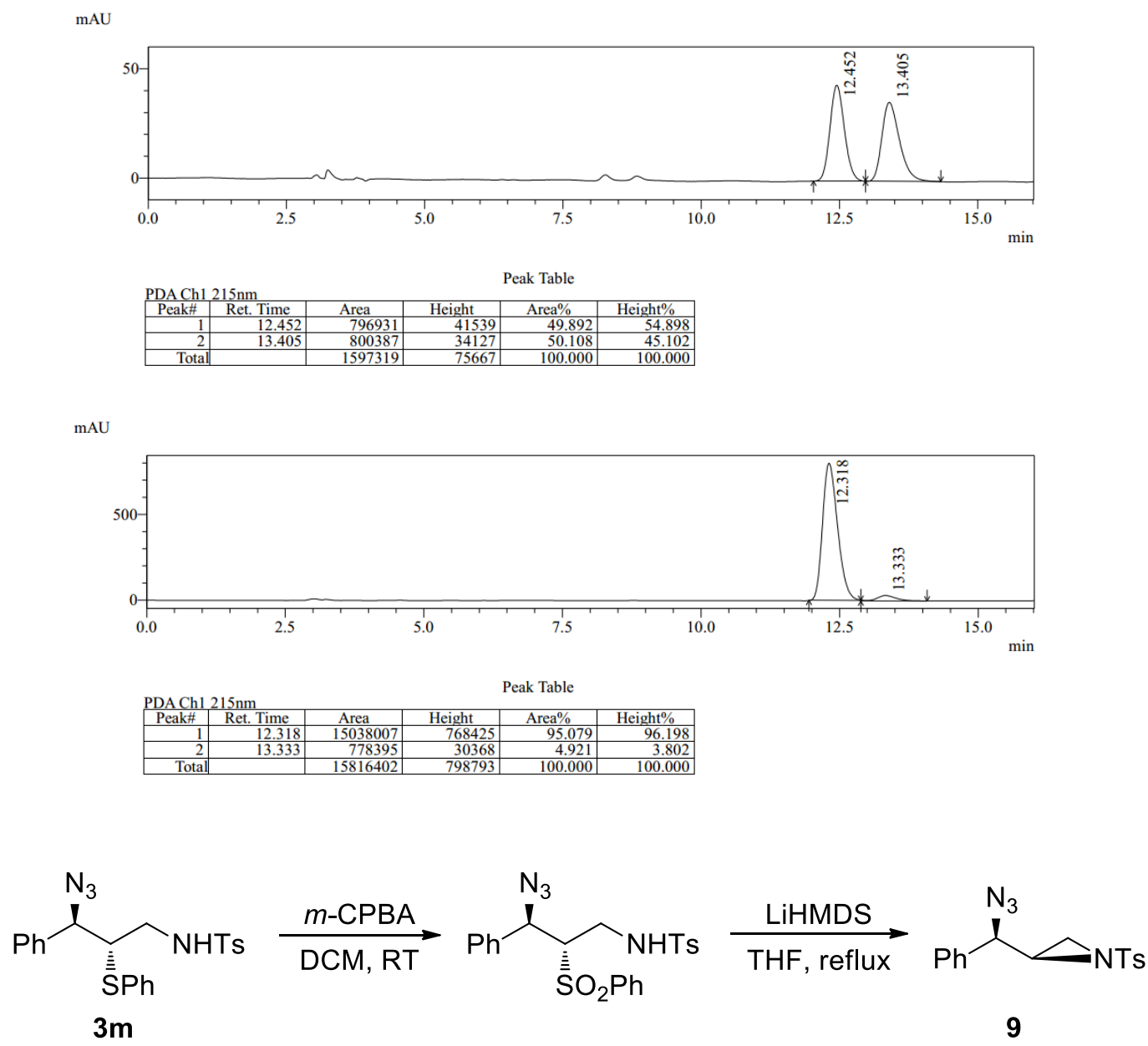

To a solution of sulfide $3 \mathrm{~m}(219 \mathrm{mg}, 0.5 \mathrm{mmol})$ in DCM $(5 \mathrm{~mL})$ was added 3-chloroperoxybenzoic acid $(215 \mathrm{mg}, 1.25 \mathrm{mmol})$ at room temperature. The solution was stirred at room temperature overnight. Then, aqueous $10 \% \mathrm{Na}_{2} \mathrm{SO}_{3}(5 \mathrm{~mL})$ was added. The mixture was stirred for $3 \mathrm{~h}$ and extracted with DCM $(5 \mathrm{~mL} \times 3)$. The organic layers were dried over $\mathrm{Na}_{2} \mathrm{SO}_{4}$, filtered, and concentrated under reduced pressure. The residue was purified by flash silica gel collumn chromotography to give the sulfone product as a white solid ( $228 \mathrm{mg}, 97 \%$ yield).

To a solution of sulfone $(94.2 \mathrm{mg}, 0.2 \mathrm{mmol})$ in dry degassed THF $(2 \mathrm{~mL})$ was droped lithium bis(trimethylsilyl)amide $(0.2 \mathrm{M}$ in THF, diluted from $1.0 \mathrm{M}$ solution, 0.4 mmol) at $-78{ }^{\circ} \mathrm{C}$ over $30 \mathrm{~min}$. The solution was stirred at $-78{ }^{\circ} \mathrm{C}$ for $30 \mathrm{~min}$ and refluxed for $24 \mathrm{~h}$ under $\mathrm{N}_{2}$ atmosphere. After cooling to room temperature, aqueous $\mathrm{NH}_{4} \mathrm{Cl}(5 \mathrm{~mL})$ was added. The mixture was stirred for $10 \mathrm{~min}$ and extracted with EtOAc $(5 \mathrm{~mL} \times 3)$. The organic layers were dried over $\mathrm{Na}_{2} \mathrm{SO}_{4}$, filtered, and concentrated under reduced pressure. The residue was purified by flash silica gel collumn chromotography to give the desired product as a colorless oil (38 $\mathrm{mg}, 73 \%$ 
yield).<smiles></smiles>

(R)-2-((R)-Azido(phenyl)methyl)-1-tosylaziridine (9): Flash column chromatography (eluent: PE/EtOAc $=30 / 1$ to $10 / 1, \mathrm{v} / \mathrm{v}$ ) to afford $\mathbf{9}$ as a colorless oil. $38 \mathrm{mg}, 71 \%$ yield (two steps) and $95 \%$ ee. $[\alpha]^{25} \mathrm{D}=-3.4\left(\mathrm{c}=0.2, \mathrm{CHCl}_{3}\right) .{ }^{1} \mathrm{H}$ NMR $\left(400 \mathrm{MHz}, \mathrm{CDCl}_{3}\right) \delta 7.84(\mathrm{~d}, J=8.2 \mathrm{~Hz}, 2 \mathrm{H}), 7.37-7.31(\mathrm{~m}, 5 \mathrm{H}), 7.29-7.24(\mathrm{~m}$, $2 \mathrm{H}), 4.22(\mathrm{~d}, J=6.8 \mathrm{~Hz}, 1 \mathrm{H}), 3.10(\mathrm{td}, J=6.8,4.7 \mathrm{~Hz}, 1 \mathrm{H}), 2.69(\mathrm{~d}, J=7.0 \mathrm{~Hz}, 1 \mathrm{H})$, $2.45(\mathrm{~s}, 3 \mathrm{H}), 2.31(\mathrm{~d}, J=4.3 \mathrm{~Hz}, 1 \mathrm{H}) .{ }^{13} \mathrm{C} \mathrm{NMR}\left(101 \mathrm{MHz}, \mathrm{CDCl}_{3}\right) \delta 145.00,135.73$, 134.62, 129.89, 129.10, 129.05, 128.29, 127.20, 65.67, 43.45, 31.00, 21.80. HR-ESI-MS $\mathrm{m} / \mathrm{z}$ calcd. for $\mathrm{C}_{16} \mathrm{H}_{16} \mathrm{~N}_{4} \mathrm{NaO}_{2} \mathrm{~S}$ [M+Na] $]^{+}$: 351.0886, found: 351.0887. HPLC (Daicel Chiralpak IA column, $i$-PrOH/hexane $=5 / 95,1 \mathrm{~mL} / \mathrm{min}, 218 \mathrm{~nm}$ ) $\mathrm{t}_{1}=$ $30.4 \min$ (major), $\mathrm{t}_{2}=35.1 \mathrm{~min}$ (minor).

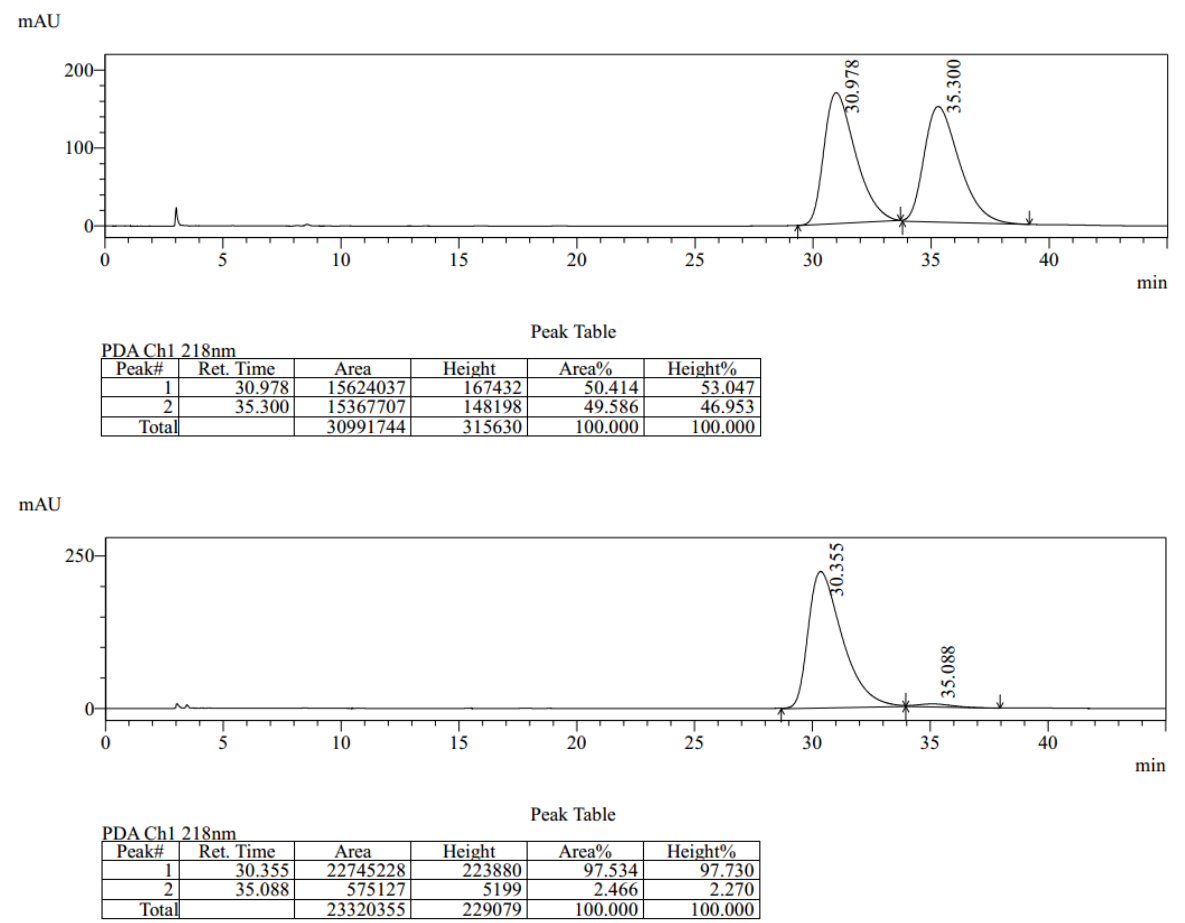

\section{Mechanistic studies}

To elucidate the reaction mechanism, some control experiments were carried out as showed below:

(a)<smiles>O=C1CC([Sn+3])CC1=O</smiles>

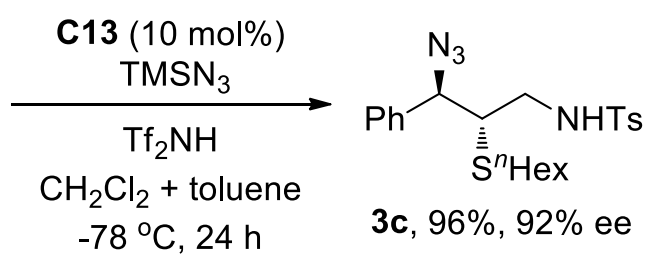


(b)<smiles>O=C1CC([Sn+][Sn])CC1=O</smiles>

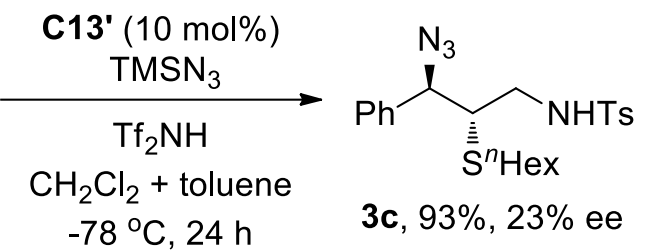

(c)

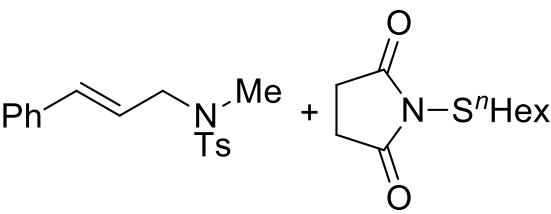

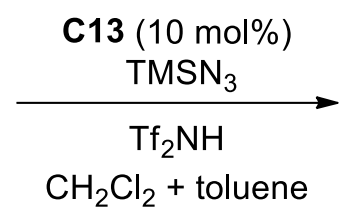

$-78{ }^{\circ} \mathrm{C}, 24 \mathrm{~h}$

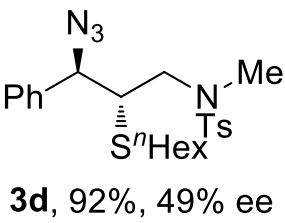<smiles>COc1c(Br)cc(Br)cc1[C@H]1Cc2ccccc2C1N[Tl]</smiles><smiles>COc1c(Br)cc(Br)cc1O[C@@H]1Cc2ccccc2C1N(C)C</smiles>

These results indicate that $\mathrm{H}$-bond donor on the substrate and the $\mathrm{N}-\mathrm{H}$ group on the catalyst play an important role for achieving high enantioselectivity of reaction.

Furthermore, thiolation products bearing an amino sulfide moiety and H-bond donor. They could serve as a Lewis base bifunctional catalysts to catalyze self thiolation reactions. To prove whether it is possible, control experiments have been carried out:
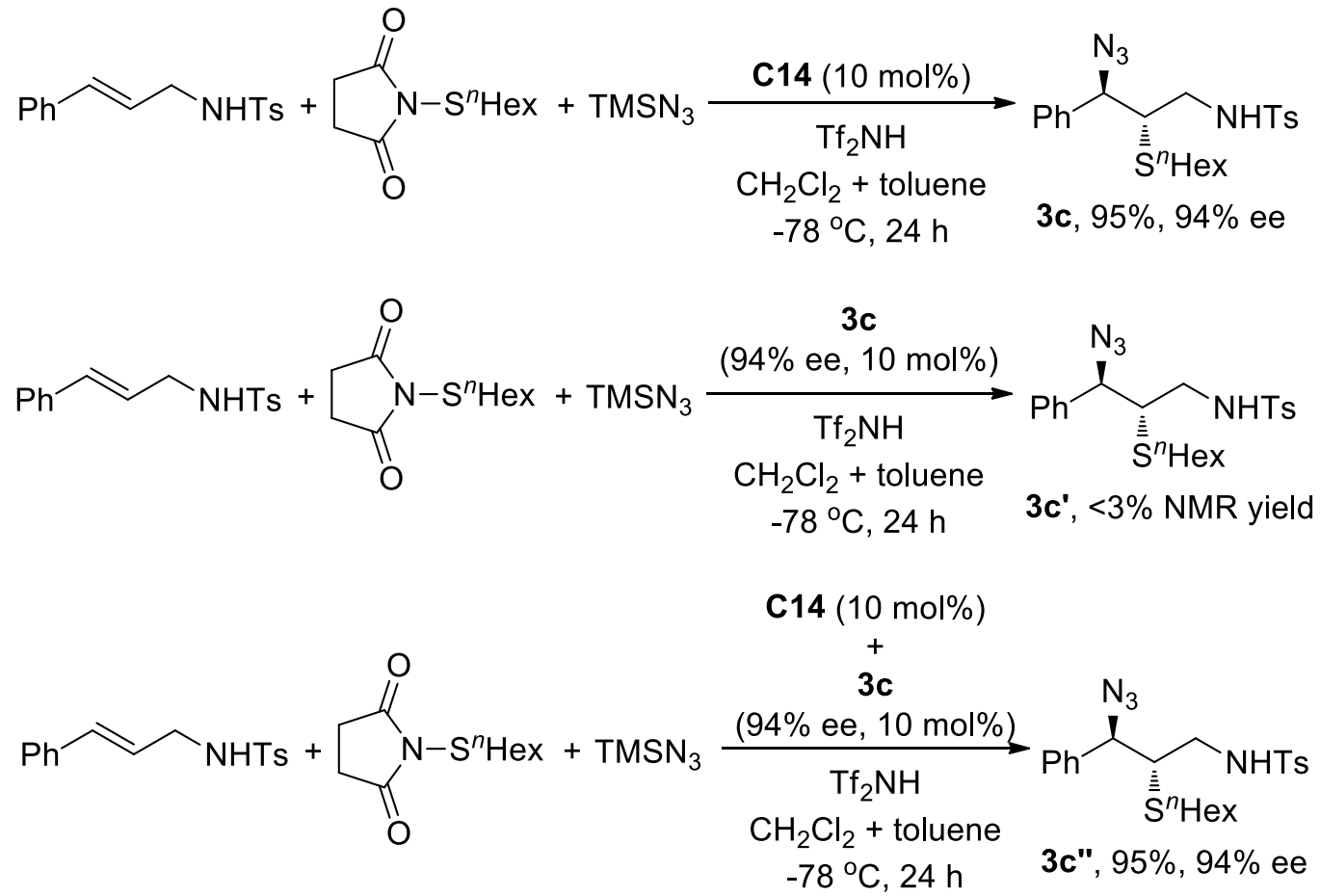
When the alkylthiolation product 3c served as a catalyst for the same reaction, difunctionalization product $\mathbf{3} \mathbf{c}^{\prime}$ was formed in only $<3 \%$ NMR yield. When the mixed catalysts of $\mathbf{C 1 4}(10 \mathrm{~mol} \%)$ and 3c (94\% ee, $10 \mathrm{~mol} \%)$ were utilized, the product was obtained with the same yield and ee as $\mathbf{3 c}$. These results indicate that alkylsulfide products do not affect the reaction.
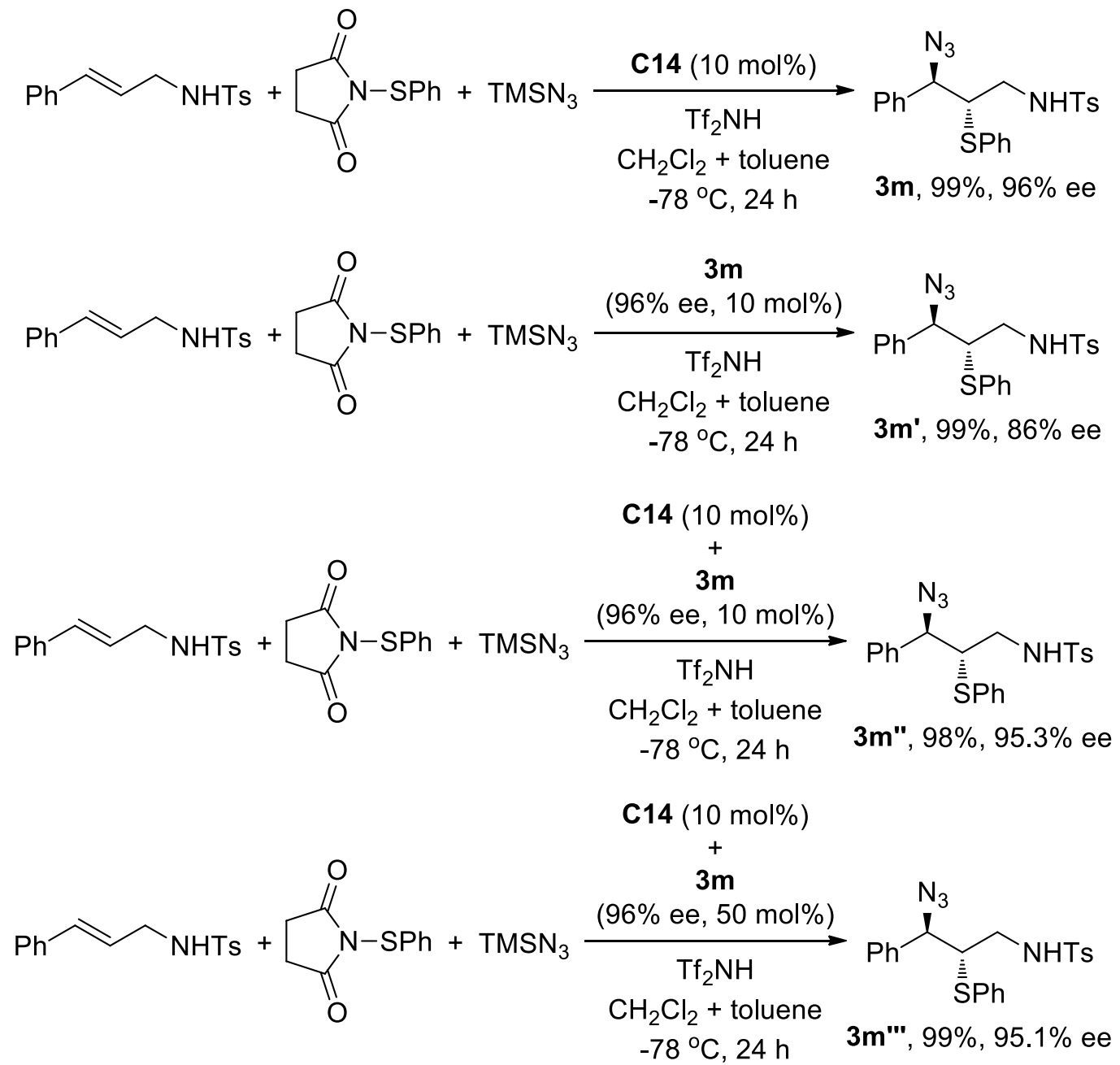

When the thiolation product $\mathbf{3 m}$ served as a catalyst for the same reaction, it was found that azidothiolation product could be formed in 99\% NMR yield with $86 \%$ ee. When the mixed catalysts of $\mathbf{C 1 4}(10 \mathrm{~mol} \%)$ and 3m (96\% ee, $10 \mathrm{~mol} \%)$ were used, product $\mathbf{3} \mathbf{m}^{\prime \prime}$ was obtained in high yield with almost same ee as $\mathbf{3 c}$. Increasing $\mathbf{3 m}$ to $50 \mathrm{~mol} \%$, the enantioselectivity was affected very slightly. These interesting results promoted us to further explore the reason.

Table S4. C14 (10 mol\%) as catalyst

\begin{tabular}{ccc}
\hline entry & time $(\min )$ & yield $^{a}(\%)$ \\
\hline 1 & 5 & 48 \\
2 & 15 & 77 \\
3 & 30 & 90 \\
\hline
\end{tabular}




\begin{tabular}{lcl}
\hline 4 & 60 & 99 \\
5 & 120 & 98 \\
6 & 180 & 98 \\
7 & 600 & 98 \\
8 & 1440 & 99 \\
\hline
\end{tabular}

${ }^{a} \overline{\text { Refers to NMR yield using quinolin as the internal standard. }}$

Table S5. 3m (10 mol\%) as catalyst

\begin{tabular}{ccc}
\hline entry & time (min) & yield $^{a}(\%)$ \\
\hline 1 & 60 & 4 \\
2 & 360 & 8 \\
3 & 720 & 30 \\
4 & 840 & 59 \\
5 & 1080 & 86 \\
6 & 1200 & 98 \\
7 & 1440 & 98 \\
\hline
\end{tabular}

${ }^{a}$ Refers to NMR yield using quinolin as the internal standard.

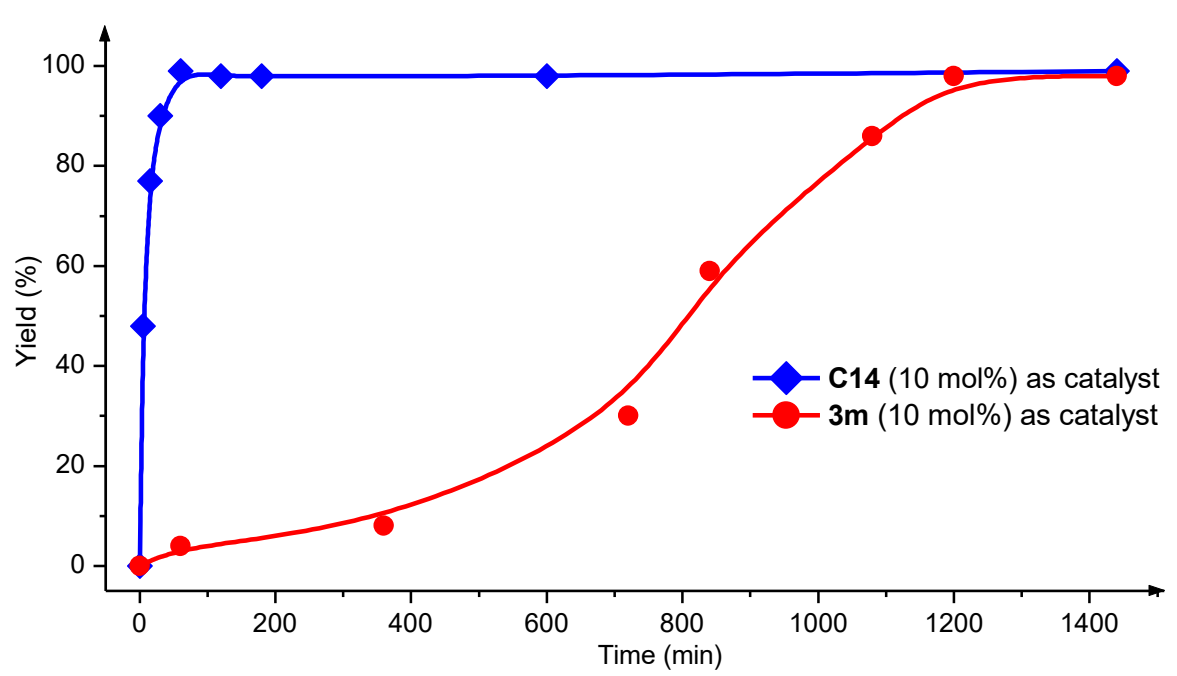

Figure S1. Correlation between yield and reaction time using C14 as catalyst and 3m as catalyst, respectively

The above results revealed that the reaction was completed in 60 min using selenide catalyst C14 (10 mol\%) and product 3m (10 mol\%) as catalyst led to only 4\% NMR yield in $60 \mathrm{~min}$. So catalyst $\mathbf{C 1 4}$ is much more efficient than $\mathbf{3 m}$ as catalyst. Accordingly, self catalysis is not remarkable.

\section{X-ray crystallographic data of 8}

Single crystals of $\mathbf{8}$ were grown by slow evaporation of its $\mathrm{CHCl}_{3} / \mathrm{PE}$ solution. Single-crystal X-ray diffraction data were collected with a 'multiwire proportional' diffractometer. The crystal was kept at $150 \mathrm{~K}$ during data collection. The structure 
was solved with the olex2.solve ${ }^{18}$ structure solution program using Charge Flipping and refined with the olex2.refine ${ }^{19}$ refinement package using Least Squares minimization. Supplementary crystallographic data have been deposited at the Cambridge Crystallographic Data Center (CCDC 1866382).

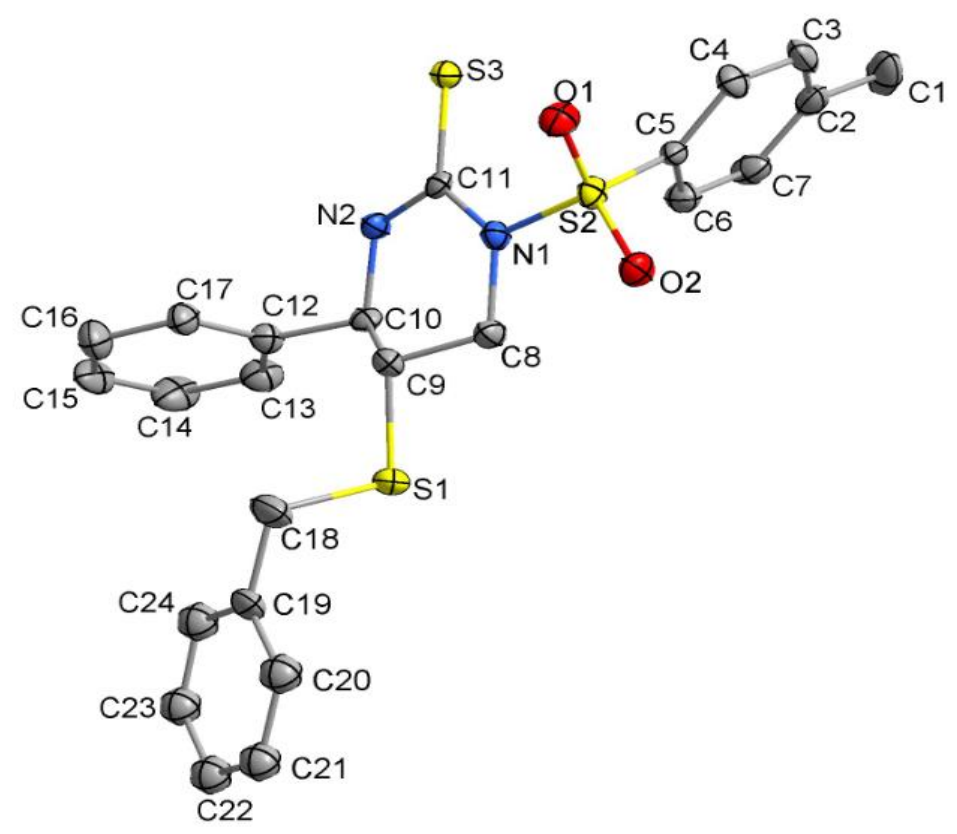

Figure S2. X-ray single crystal structure of 8

Table S6. Crystal data and structure refinement for 8

Empirical formula

Formula weight

Temperature/K

Crystal system

Space group

$\mathrm{a} / \AA$

$\mathrm{b} / \AA$

$\mathrm{c} / \AA$

$\alpha /^{\circ}$

$\beta /{ }^{\circ}$

$\gamma /{ }^{\circ}$

Volume $/ \AA^{3}$

Z

$\rho($ calc $) \mathrm{g} / \mathrm{cm}^{3}$

$\mu(\mathrm{CuKa}) / \mathrm{mm}^{-1}$

$\mathrm{F}(000)$

Crystal size $/ \mathrm{mm}^{3}$

Radiation

$2 \Theta$ range for data collection/ ${ }^{\circ}$

Index ranges
$\mathrm{C}_{24} \mathrm{H}_{24} \mathrm{~N}_{2} \mathrm{O}_{2} \mathrm{~S}_{3}$

468.10

150

Triclinic

$P 1$

$5.70840(10)$

14.6112(2)

$15.7856(2)$

$72.8010(10)$

$87.8770(10)$

$87.3440(10)$

1256.02(3)

2

1.333

4.367

502.0

$0.5 \times 0.1 \times 0.05$

$\mathrm{CuK} \alpha(\lambda=1.54178)$

7.256 to 125.978

$-6 \leq \mathrm{h} \leq 7,-18 \leq \mathrm{k} \leq 18,-19 \leq 1 \leq 19$ 
Reflections collected

Independent reflections

Data/restraints/parameters

Goodness-of-fit on $\mathrm{F}^{2}$

Final $\mathrm{R}$ indexes $[\mathrm{I}>=2 \sigma(\mathrm{I})]$

Final $\mathrm{R}$ indexes [all data]

Largest diff. peak/hole / e $\AA^{-3}$

Flack parameter
43701

$9469\left[\mathrm{R}_{\text {int }}=0.0430, \mathrm{R}_{\text {sigma }}=0.00317\right]$

9469/3/597

1.083

$\mathrm{R}_{1}=0.0548, \mathrm{wR}_{2}=0.1520$

$\mathrm{R}_{1}=0.0576, \mathrm{wR}_{2}=0.1558$

$1.09,-0.84$

$0.002(11)$

Table S7. Bond lengths for 8

\begin{tabular}{|c|c|c|c|c|c|}
\hline Atom & Atom & Length/Å & Atom & Atom & Length/Å \\
\hline $\mathrm{S} 2$ & $\mathrm{O} 1$ & $1.433(5)$ & $\mathrm{C} 4$ & C3 & $1.395(9)$ \\
\hline $\mathrm{S} 2$ & $\mathrm{O} 2$ & $1.425(4)$ & $\mathrm{C} 10$ & $\mathrm{C} 12$ & $1.520(7)$ \\
\hline $\mathrm{S} 2$ & N1 & $1.690(4)$ & C6 & $\mathrm{C} 7$ & $1.391(8)$ \\
\hline $\mathrm{S} 2$ & $\mathrm{C} 5$ & $1.764(6)$ & $\mathrm{C} 15$ & C14 & $1.365(11)$ \\
\hline $\mathrm{S} 3$ & C11 & $1.695(6)$ & $\mathrm{C} 15$ & C16 & $1.388(11)$ \\
\hline $\mathrm{S} 1$ & C9 & $1.828(6)$ & $\mathrm{C} 2$ & $\mathrm{C} 7$ & $1.394(9)$ \\
\hline $\mathrm{S} 1$ & C18 & $1.824(7)$ & $\mathrm{C} 2$ & C3 & $1.378(10)$ \\
\hline $\mathrm{N} 2$ & $\mathrm{C} 11$ & $1.337(7)$ & $\mathrm{C} 2$ & $\mathrm{C} 1$ & $1.510(8)$ \\
\hline $\mathrm{N} 2$ & $\mathrm{C} 10$ & $1.472(7)$ & $\mathrm{C} 12$ & $\mathrm{C} 17$ & $1.375(9)$ \\
\hline N1 & C11 & $1.377(6)$ & C19 & $\mathrm{C} 20$ & $1.379(10)$ \\
\hline N1 & $\mathrm{C} 8$ & $1.496(7)$ & C19 & C18 & $1.500(9)$ \\
\hline C13 & $\mathrm{C} 12$ & $1.390(9)$ & C19 & $\mathrm{C} 24$ & $1.362(11)$ \\
\hline C13 & $\mathrm{C} 14$ & $1.392(9)$ & $\mathrm{C} 17$ & $\mathrm{C} 16$ & $1.394(8)$ \\
\hline $\mathrm{C} 8$ & C9 & $1.517(7)$ & $\mathrm{C} 24$ & $\mathrm{C} 23$ & $1.379(18)$ \\
\hline $\mathrm{C} 5$ & $\mathrm{C} 4$ & $1.384(8)$ & $\mathrm{C} 20$ & $\mathrm{C} 21$ & $1.405(13)$ \\
\hline $\mathrm{C} 5$ & C6 & $1.373(9)$ & $\mathrm{C} 21$ & $\mathrm{C} 22$ & $1.320(2)$ \\
\hline C9 & $\mathrm{C} 10$ & $1.524(7)$ & $\mathrm{C} 22$ & $\mathrm{C} 23$ & $1.380(3)$ \\
\hline
\end{tabular}

Table S8. Bond angles for 8

\begin{tabular}{|c|c|c|c|c|c|c|c|}
\hline Atom & Atom & Atom & Angle $/^{\circ}$ & Atom & Atom & Atom & Angle $/^{\circ}$ \\
\hline $\mathrm{O} 1$ & S2 & N1 & $110.6(3)$ & $\mathrm{N} 2$ & $\mathrm{C} 10$ & $\mathrm{C} 12$ & $109.3(4)$ \\
\hline O1 & S2 & $\mathrm{C} 5$ & $109.9(3)$ & $\mathrm{C} 12$ & $\mathrm{C} 10$ & C9 & $114.5(4)$ \\
\hline $\mathrm{O} 2$ & S2 & $\mathrm{O} 1$ & 118.1(3) & $\mathrm{C} 5$ & C6 & $\mathrm{C} 7$ & $118.7(5)$ \\
\hline $\mathrm{O} 2$ & S2 & N1 & $103.7(2)$ & C14 & $\mathrm{C} 15$ & C16 & $120.6(6)$ \\
\hline $\mathrm{O} 2$ & S2 & $\mathrm{C} 5$ & $108.6(2)$ & $\mathrm{C} 7$ & $\mathrm{C} 2$ & $\mathrm{C} 1$ & $120.3(7)$ \\
\hline N1 & S2 & $\mathrm{C} 5$ & $105.1(2)$ & $\mathrm{C} 3$ & $\mathrm{C} 2$ & $\mathrm{C} 7$ & 119.1(6) \\
\hline $\mathrm{C} 18$ & $\mathrm{~S} 1$ & C9 & $100.2(3)$ & $\mathrm{C} 3$ & $\mathrm{C} 2$ & $\mathrm{C} 1$ & $120.6(6)$ \\
\hline C11 & $\mathrm{N} 2$ & C10 & $126.8(4)$ & C13 & C12 & C10 & $118.0(5)$ \\
\hline
\end{tabular}




\begin{tabular}{|c|c|c|c|c|c|c|c|}
\hline $\mathrm{C} 11$ & N1 & $\mathrm{S} 2$ & $122.0(4)$ & $\mathrm{C} 17$ & $\mathrm{C} 12$ & $\mathrm{C} 13$ & $120.3(5)$ \\
\hline C11 & N1 & $\mathrm{C} 8$ & $122.4(4)$ & $\mathrm{C} 17$ & $\mathrm{C} 12$ & $\mathrm{C} 10$ & $121.7(5)$ \\
\hline $\mathrm{C} 8$ & $\mathrm{~N} 1$ & $\mathrm{~S} 2$ & $114.8(3)$ & $\mathrm{C} 20$ & C19 & C18 & $118.7(7)$ \\
\hline $\mathrm{N} 2$ & $\mathrm{C} 11$ & S3 & 119.1(4) & $\mathrm{C} 24$ & C19 & $\mathrm{C} 20$ & $119.8(8)$ \\
\hline $\mathrm{N} 2$ & $\mathrm{C} 11$ & N1 & $117.1(5)$ & $\mathrm{C} 24$ & C19 & $\mathrm{C} 18$ & $121.5(8)$ \\
\hline N1 & $\mathrm{C} 11$ & S3 & $123.8(4)$ & C6 & $\mathrm{C} 7$ & $\mathrm{C} 2$ & $120.6(6)$ \\
\hline C12 & $\mathrm{C} 13$ & $\mathrm{C} 14$ & $119.8(6)$ & $\mathrm{C} 15$ & $\mathrm{C} 14$ & $\mathrm{C} 13$ & $119.9(6)$ \\
\hline $\mathrm{N} 1$ & $\mathrm{C} 8$ & $\mathrm{C} 9$ & $110.0(4)$ & $\mathrm{C} 2$ & $\mathrm{C} 3$ & $\mathrm{C} 4$ & $121.2(5)$ \\
\hline $\mathrm{C} 4$ & $\mathrm{C} 5$ & S2 & $117.1(5)$ & $\mathrm{C} 12$ & $\mathrm{C} 17$ & $\mathrm{C} 16$ & $119.6(6)$ \\
\hline C6 & $\mathrm{C} 5$ & $\mathrm{~S} 2$ & $120.5(4)$ & $\mathrm{C} 15$ & $\mathrm{C} 16$ & $\mathrm{C} 17$ & $119.8(7)$ \\
\hline C6 & $\mathrm{C} 5$ & $\mathrm{C} 4$ & $122.2(5)$ & C19 & $\mathrm{C} 20$ & $\mathrm{C} 21$ & $119.7(10)$ \\
\hline $\mathrm{C} 8$ & $\mathrm{C} 9$ & $\mathrm{~S} 1$ & $107.1(4)$ & C19 & $\mathrm{C} 18$ & $\mathrm{~S} 1$ & $109.0(5)$ \\
\hline $\mathrm{C} 8$ & C9 & $\mathrm{C} 10$ & $109.5(4)$ & C19 & $\mathrm{C} 24$ & $\mathrm{C} 23$ & $120.2(12)$ \\
\hline $\mathrm{C} 10$ & C9 & $\mathrm{S} 1$ & $111.0(4)$ & $\mathrm{C} 22$ & $\mathrm{C} 21$ & $\mathrm{C} 20$ & $119.6(12)$ \\
\hline $\mathrm{C} 5$ & $\mathrm{C} 4$ & $\mathrm{C} 3$ & 118.1(6) & $\mathrm{C} 21$ & $\mathrm{C} 22$ & $\mathrm{C} 23$ & $121.5(9)$ \\
\hline $\mathrm{N} 2$ & $\mathrm{C} 10$ & C9 & $106.6(4)$ & $\mathrm{C} 24$ & $\mathrm{C} 23$ & $\mathrm{C} 22$ & $119.3(12)$ \\
\hline
\end{tabular}

\section{References}

(1) Chen, C.; Jin, S.; Zhang, Z.; Wei, B.; Wang, H.; Zhang, K.; Lv, H.; Dong, X.-Q.; Zhang, X. Rhodium/Yanphos-Catalyzed Asymmetric Interrupted Intramolecular Hydroaminomethylation of trans-1,2-Disubstituted Alkenes. J. Am. Chem. Soc. 2016, 138, 9017-9020.

(2) Sorimachi, K.; Terada, M. Relay Catalysis by a Metal-Complex/Brønsted Acid Binary System in a Tandem Isomerization/Carbon-Carbon Bond Forming Sequence. J. Am. Chem. Soc. 2008, 130, 14452-14453.

(3) Zhang, W.; Liu, N.; Schienebeck, C. M.; Zhou, X.; Izhar, I. I.; Guzei, I. A.; Tang, W. Enantioselective Intermolecular Bromoesterification of Allylic Sulfonamides. Chem. Sci. 2013, 4, 2652-2656.

(4) Chogii, I.; Das, P.; Fell, J. S.; Scott, K. A.; Crawford, M. N.; Houk, K. N.; Njardarson, J. T. New Class of Anion-Accelerated Amino-Cope Rearrangements as Gateway to Diverse Chiral Structures. J. Am. Chem. Soc. 2017, 139, 13141-13146.

(5) Huang, J.; Zheng, J.; Wu, W.; Li, J.; Ma, Z.; Ren, Y.; Jiang, H. Palladium-Catalyzed Intermolecular Oxidative Cyclization of Allyltosylamides with AcOH: Assembly of 3-Pyrrolin-2-ones. J. Org. Chem. 2017, 82, 8191-8198.

(6) Ashikari, Y.; Nokami, T.; Yoshida, J. Integrated Electrochemical-Chemical Oxidation Mediated by Alkoxysulfonium Ions. J. Am. Chem. Soc. 2011, 133, 11840-11843.

(7) Arai, S.; Koike, Y.; Hada, H.; Nishida, A. Catalytic Dicyanative [4+2] Cycloaddition Triggered by Cyanopalladation Using Ene-Enynes and Cyclic Enynes with Methyl Acrylate. J. Org. Chem. 2010, 75, 7573-7579. 
(8) Ramesh, E.; Guntreddi, T.; Sahoo, A. K. $\mathrm{AlCl}_{3}$-Catalyzed Intermolecular Annulation of Thiol Derivatives and Alkynes by 1,2-Sulfur Migration: Construction of 6-Substituted Benzo[b]thiophenes. Eur. J. Org. Chem. 2017, 4405-4413.

(9) Hostier, T.; Ferey, V.; Ricci, G.; Pardo, D. G.; Cossy, J. Synthesis of Aryl Sulfides: Metal-Free C-H Sulfenylation of Electron-Rich Arenes. Org. Lett. 2015, 17, 3898-3901.

(10) Ramesh, E.; Shankar, M.; Dana, S.; Sahoo, A. K. Silver-Mediated Oxidative Annulation of $N$-Arylthio Succinimides with Alkynes: Direct Access to Benzo[b]thiophenes. Org. Chem. Front. 2016, 3, 1126-1130.

(11) Sheppard, J. G.; Long, T. E. Allicin-Inspired Thiolated Fluoroquinolones as Antibacterials Against ESKAPE Pathogens. Bioorg. Med. Chem. Lett. 2016, 26, $5545-5549$.

(12) Reese, C. B.; Yan, H. B. Solution Phase Synthesis of ISIS 2922 (Vitravene) by the Modified H-phosphonate Approach. J. Chem. Soc., Perkin Trans. 1, 2002, 2619-2633.

(13) Abbasi, M.; Jabbari, A. One-Pot Conversion of Alkyl Halides to Organic Disulfides (Disulfanes) Using Thiourea and Hexamethyldisilazane (HMDS) in DMSO. J. Iran. Chem. Soc. 2016, 13, 81-86.

(14) Harusawa, S.; Yoshida, K.; Kojima, C.; Araki, L.; Kurihara, T. Design and Synthesis of an Aminobenzo-15-crown-5-labeled Estradiol Tethered with Disulfide Linkage. Tetrahedron 2004, 60, 11911-11922.

(15) Luca, L. D.; Giacomelli, G.; Porcheddu, A. An Efficient Route to Alkyl Chlorides from Alcohols Using the Complex TCT/DMF. Org. Lett. 2002, 4, 553-555.

(16) Luo, J.; Liu, Y.; Zhao, X. Chiral Selenide-Catalyzed Enantioselective Construction of Saturated Trifluoromethylthiolated Azaheterocycles. Org. Lett. 2017, 19, 3434-3437.

(17) Bellavista, T.; Meninno, S.; Lattanzi, A.; Sala, G. D. Asymmetric Hydroazidation of Nitroalkenes Promoted by a Secondary Amine-Thiourea Catalyst. Adv. Synth. Catal. 2015, 357, 3365-3373.

(18) Dolomanov, O. V.; Bourhis, L. J.; Gildea, R. J.; Howard, J. A. K.; Puschmann, H. OLEX2: A Complete Structure Solution, Refinement and Analysis Program. J. Appl. Cryst. 2009, 42, 339-341.

(19) Bourhis, L. J.; Dolomanov, O. V.; Gildea, R. J.; Howard, J. A. K.; Puschmann, H. The Anatomy of a Comprehensive Constrained, Restrained Refinement Program for the Modern Computing Environment-Olex2 Dissected. Acta Cryst. 2015, A71, $59-75$. 


\section{NMR spectra of new compounds}

${ }^{1} \mathrm{H}$ NMR (400 MHz, $\mathrm{CDCl}_{3}$ ) spectrum of compound $\mathbf{1 v}$

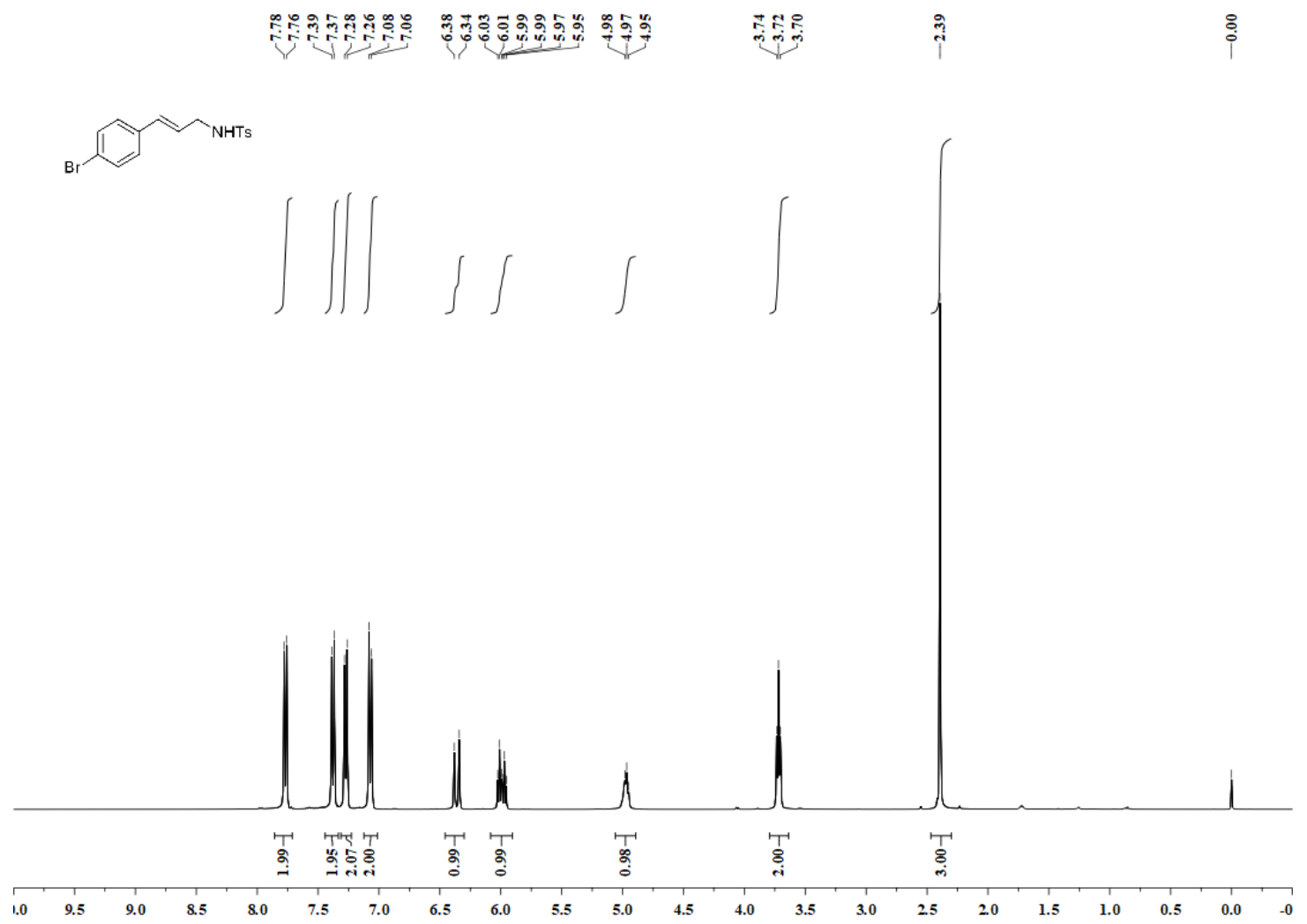

${ }^{13} \mathrm{C}$ NMR (101 MHz, $\left.\mathrm{CDCl}_{3}\right)$ spectrum of compound $\mathbf{1 v}$

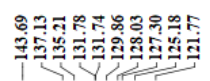

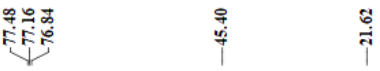

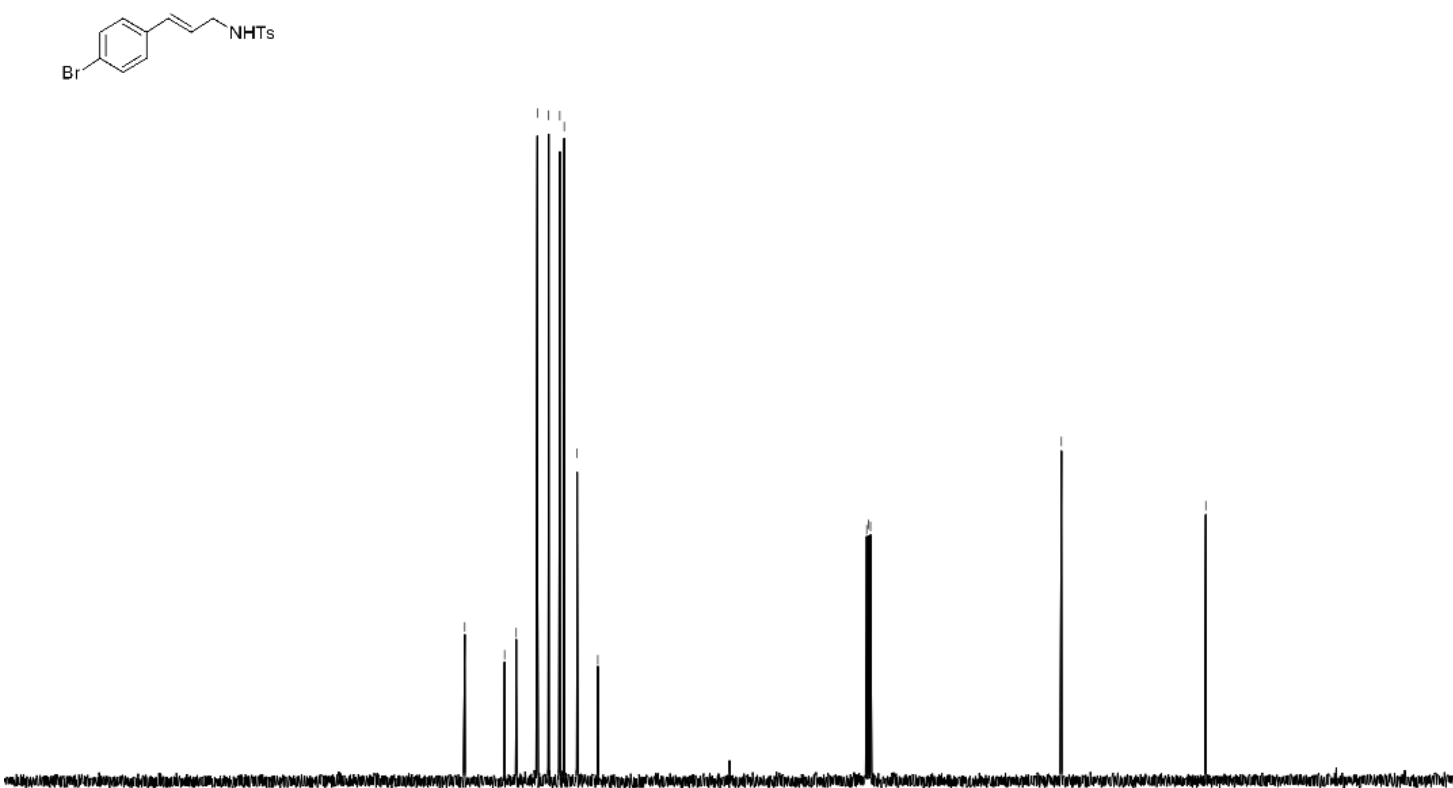

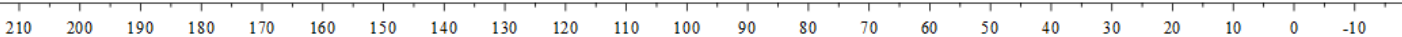


${ }^{1} \mathrm{H}$ NMR (400 MHz, $\mathrm{CDCl}_{3}$ ) spectrum of compound $\mathbf{1 x}$

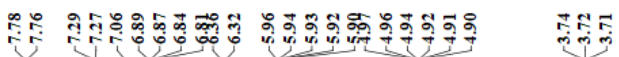

NHTS
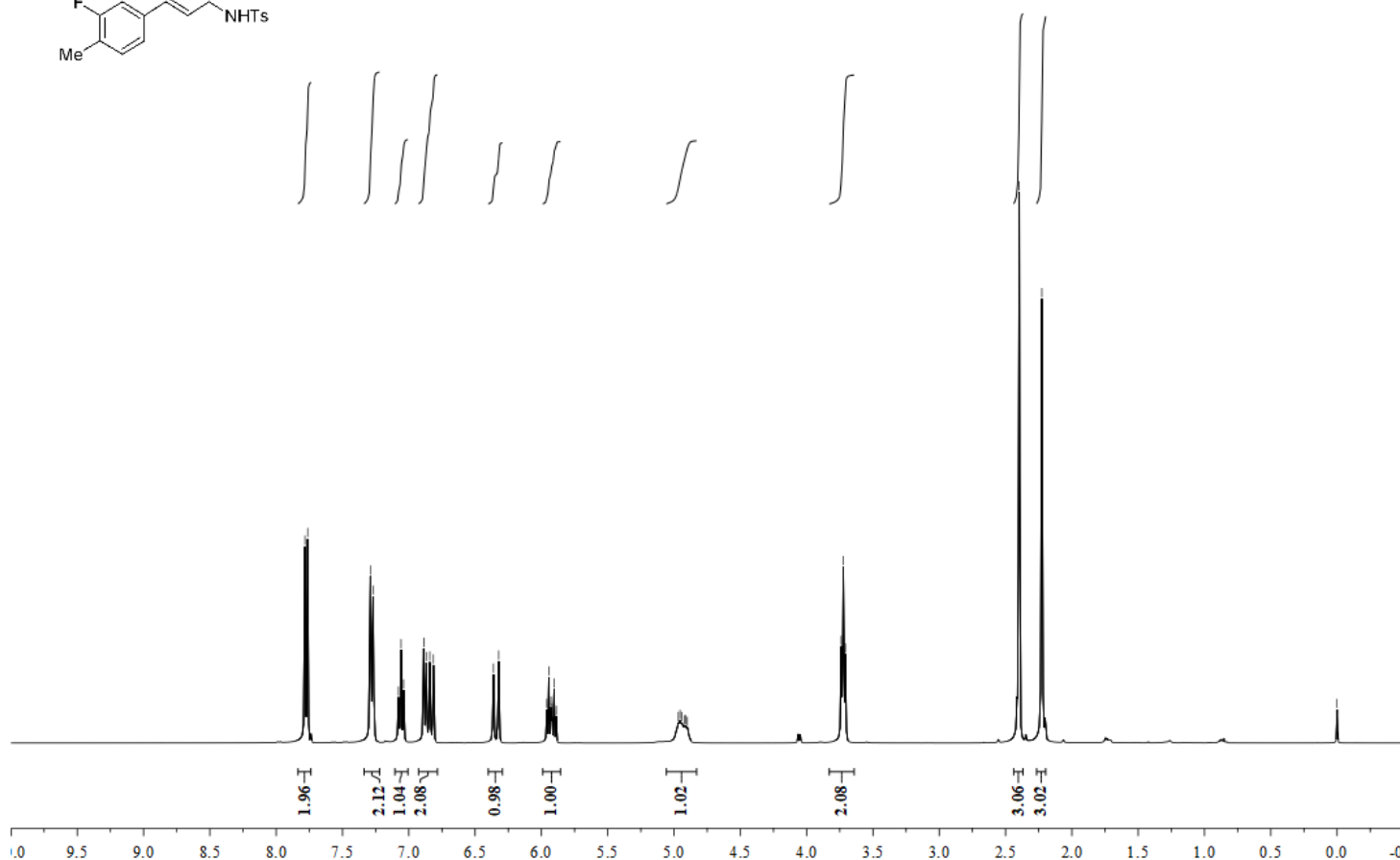

${ }^{13} \mathrm{C}$ NMR (101 MHz, $\mathrm{CDCl}_{3}$ ) spectrum of compound $\mathbf{1 x}$

\begin{tabular}{|c|c|}
\hline 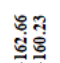 & 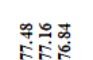 \\
\hline
\end{tabular}

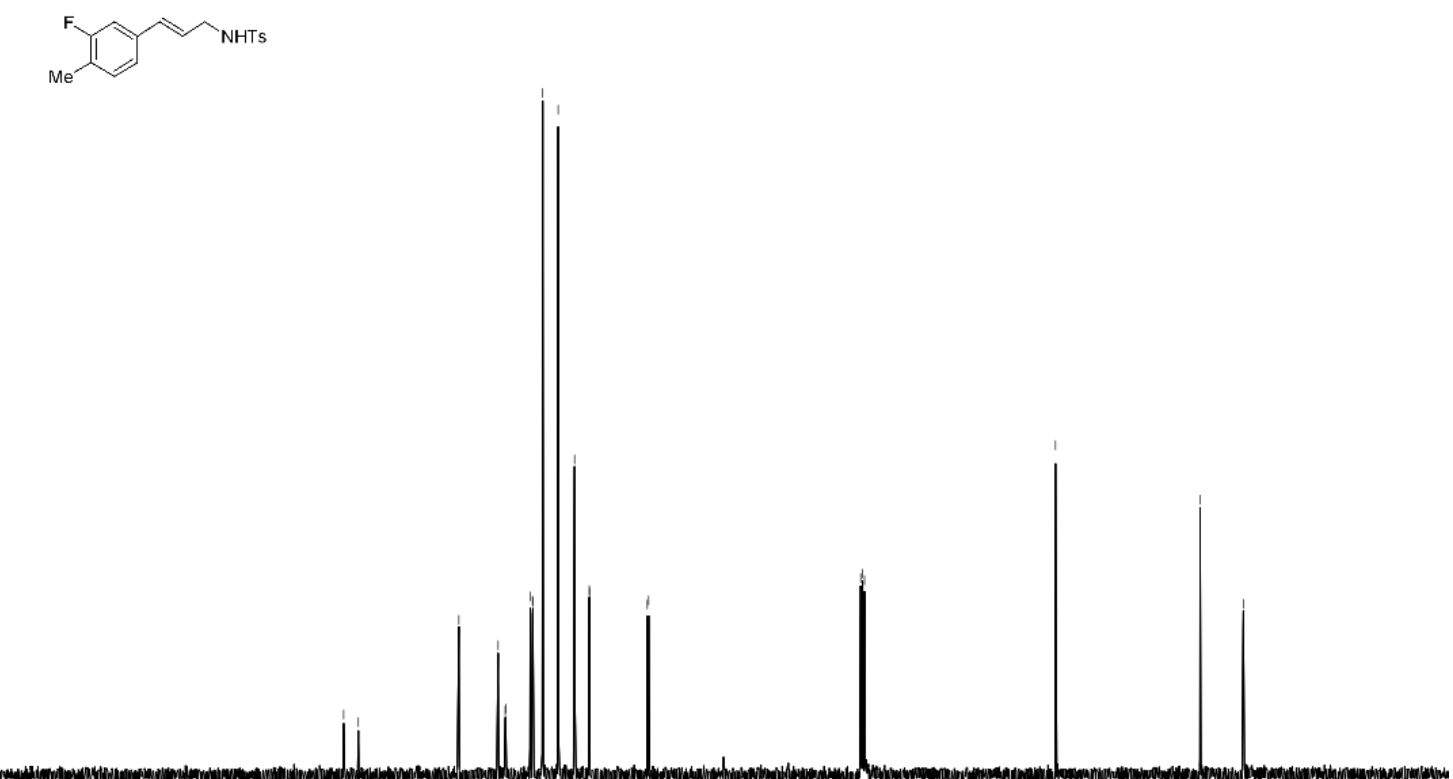

$\begin{array}{llllllllllllllllllllll}210 & 200 & 190 & 180 & 170 & 160 & 150 & 140 & 130 & 120 & 110 & 100 & 90 & 80 & 70 & 60 & 50 & 40 & 30 & 20 & 10 & 0\end{array}$ 
${ }^{1} \mathrm{H}$ NMR (400 MHz, $\mathrm{CDCl}_{3}$ ) spectrum of compound $\mathbf{1 a b}$

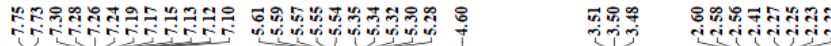
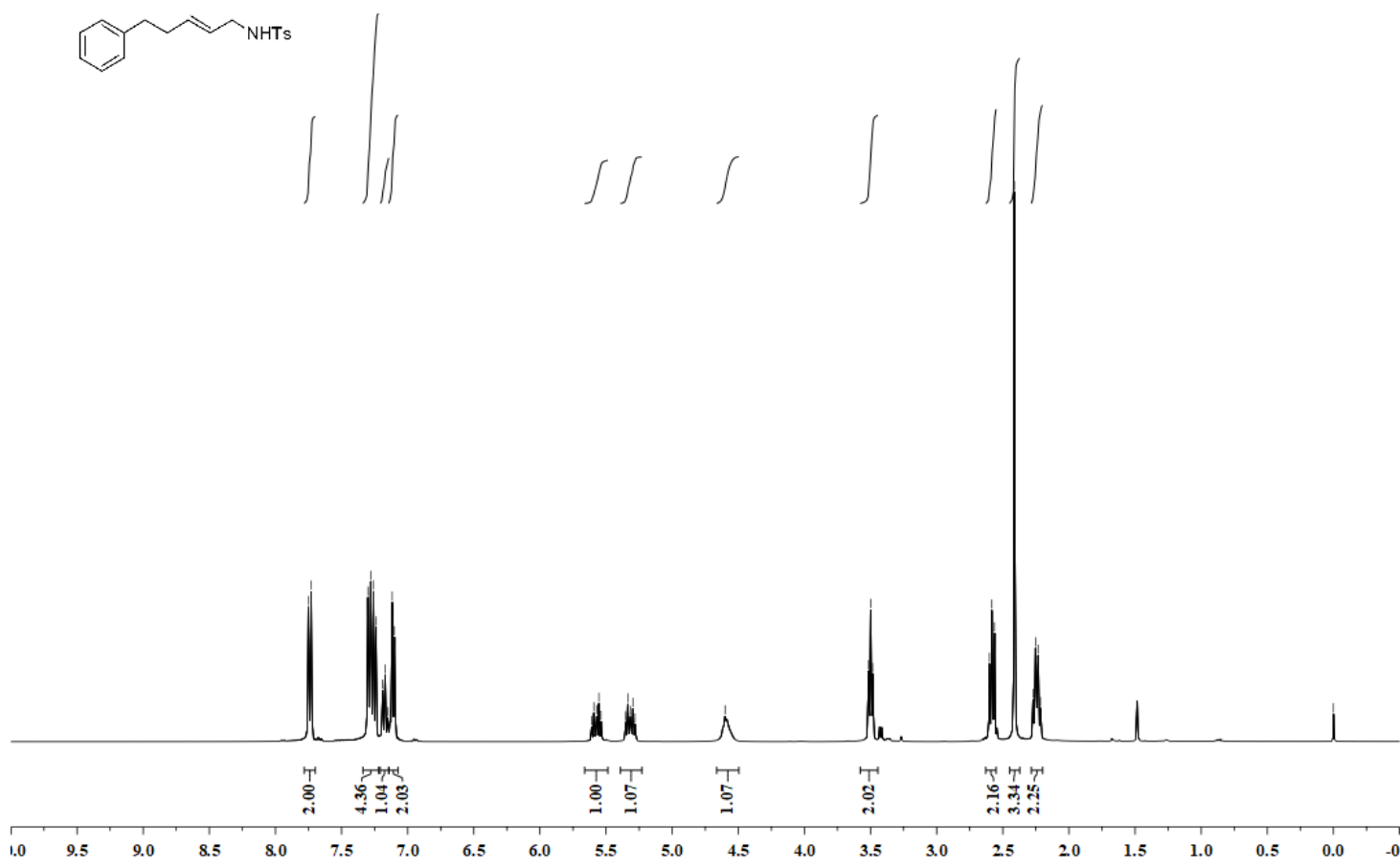

${ }^{13} \mathrm{C}$ NMR $\left(101 \mathrm{MHz}, \mathrm{CDCl}_{3}\right)$ spectrum of compound 1ab

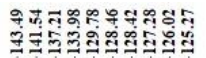

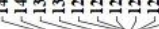<smiles>SNCC=CCCc1ccccc1</smiles>

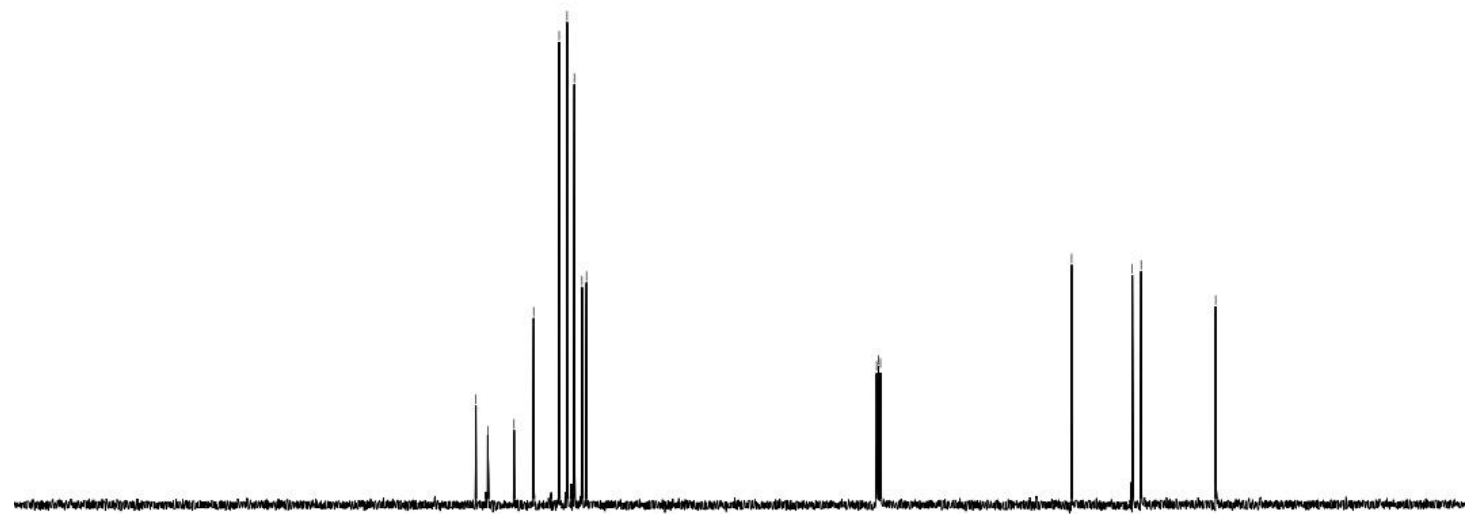


${ }^{1} \mathrm{H}$ NMR (400 MHz, $\mathrm{CDCl}_{3}$ ) spectrum of compound 2a

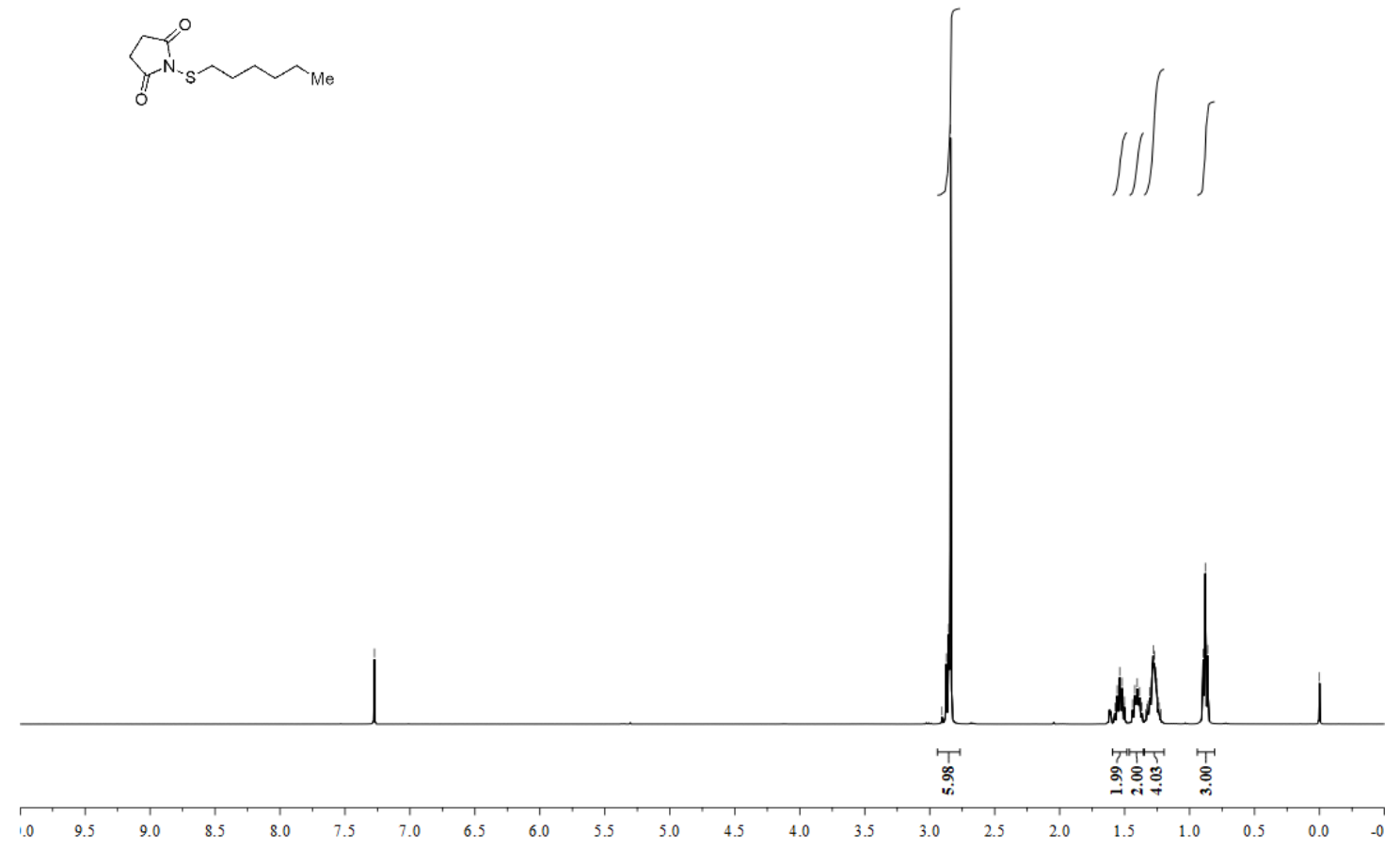

${ }^{13} \mathrm{C}$ NMR $\left(101 \mathrm{MHz}, \mathrm{CDCl}_{3}\right)$ spectrum of compound $\mathbf{2 a}$

$\stackrel{4}{\stackrel{4}{\leftrightarrows}}$
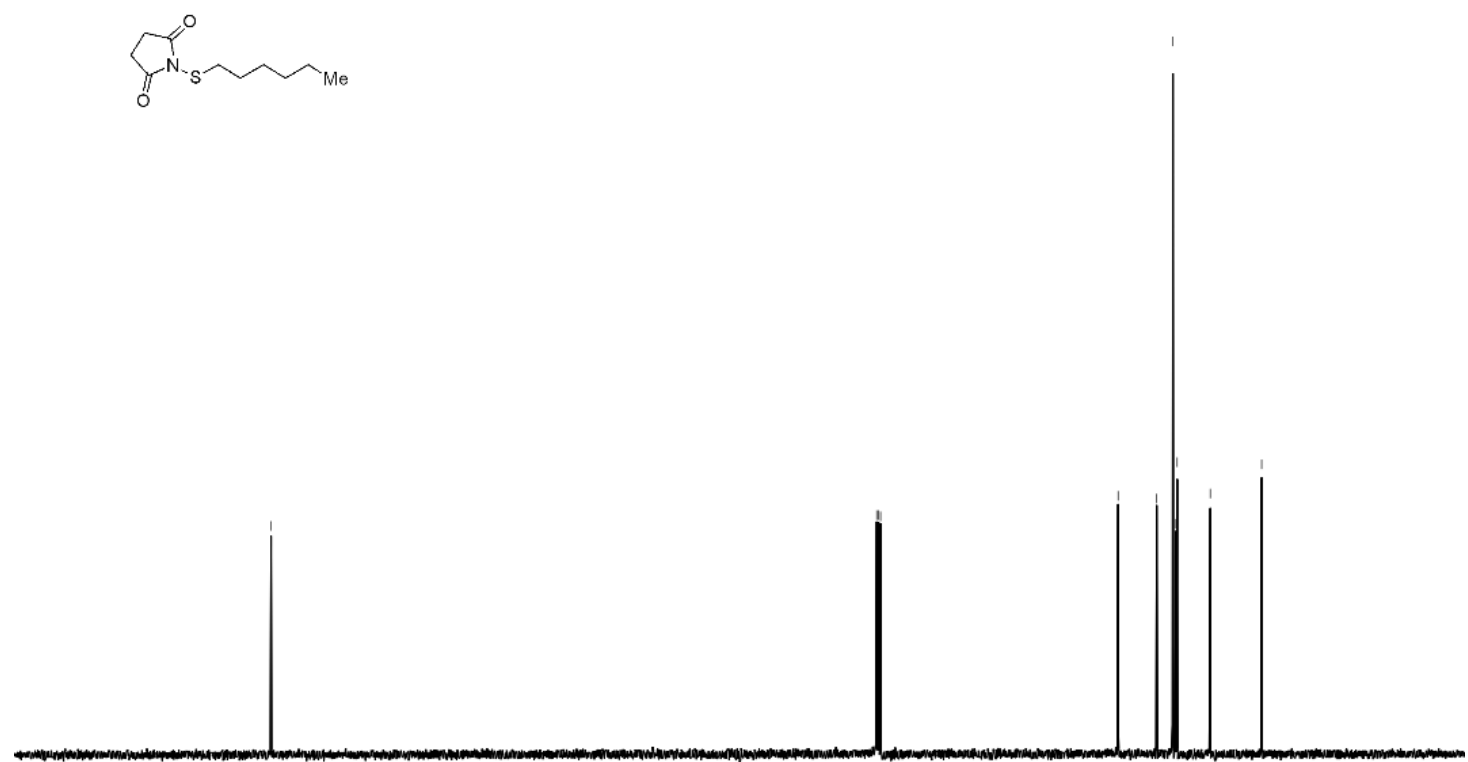

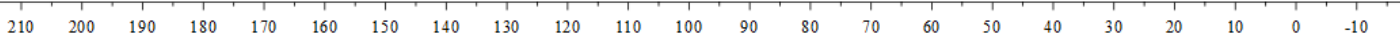


${ }^{1} \mathrm{H}$ NMR (400 MHz, $\mathrm{CDCl}_{3}$ ) spectrum of compound $\mathbf{2 b}$

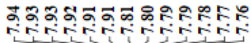

कom toำ

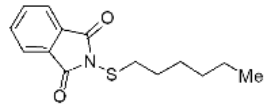

$\|$
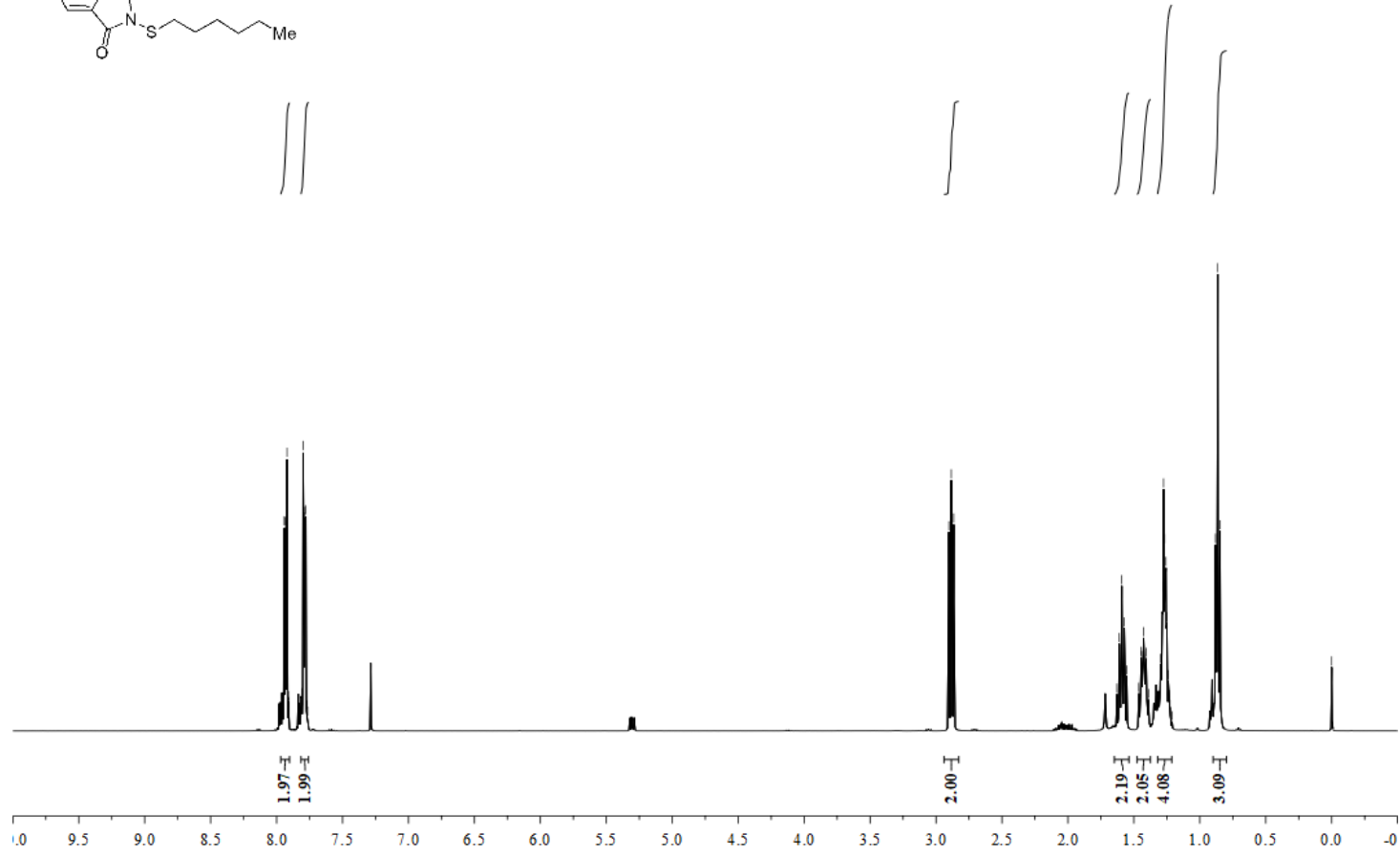

${ }^{13} \mathrm{C}$ NMR (101 MHz, $\mathrm{CDCl}_{3}$ ) spectrum of compound $\mathbf{2 b}$

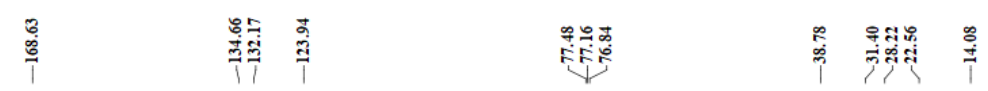

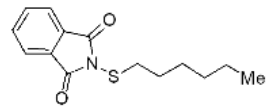

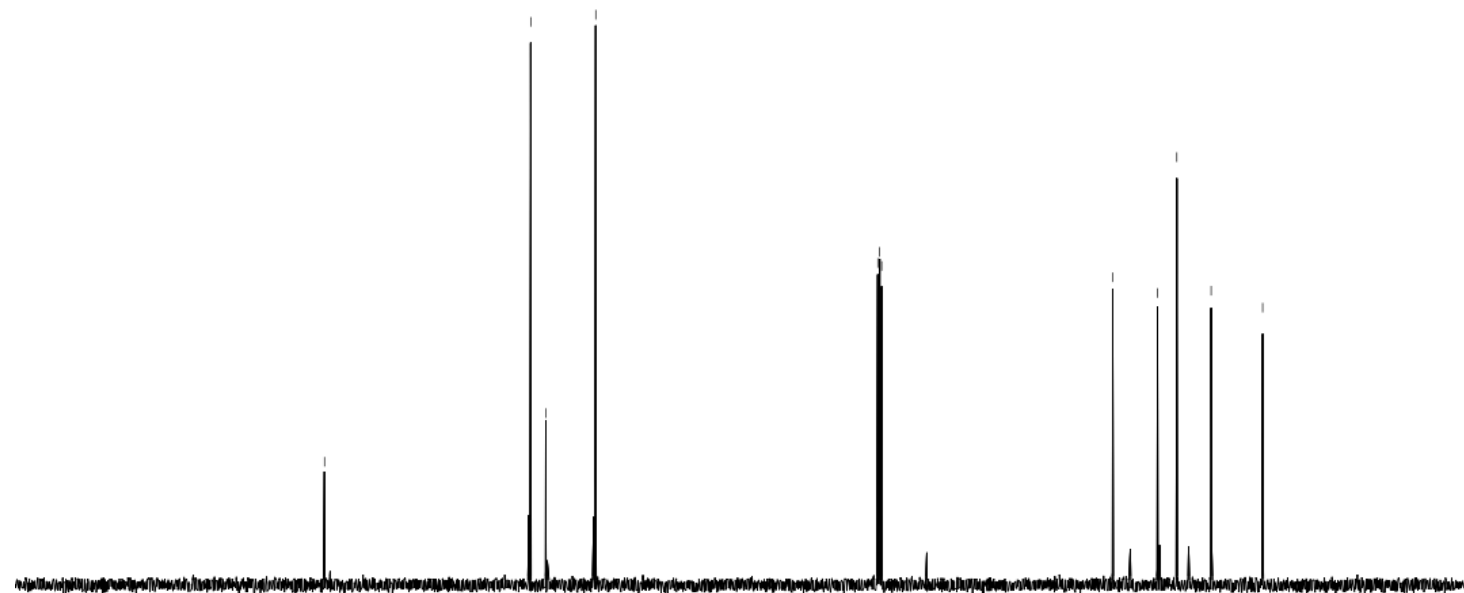

$\begin{array}{llllllllllllllllllllll}210 & 200 & 190 & 180 & 170 & 160 & 150 & 140 & 130 & 120 & 110 & 100 & 90 & 80 & 70 & 60 & 50 & 40 & 30 & 20 & 10 & 0\end{array}$ 
${ }^{1} \mathrm{H}$ NMR (400 MHz, $\mathrm{CDCl}_{3}$ ) spectrum of compound 2c

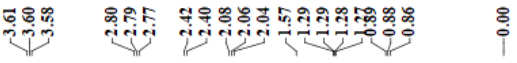

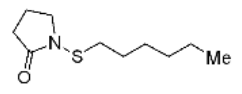

$\iint|d|$

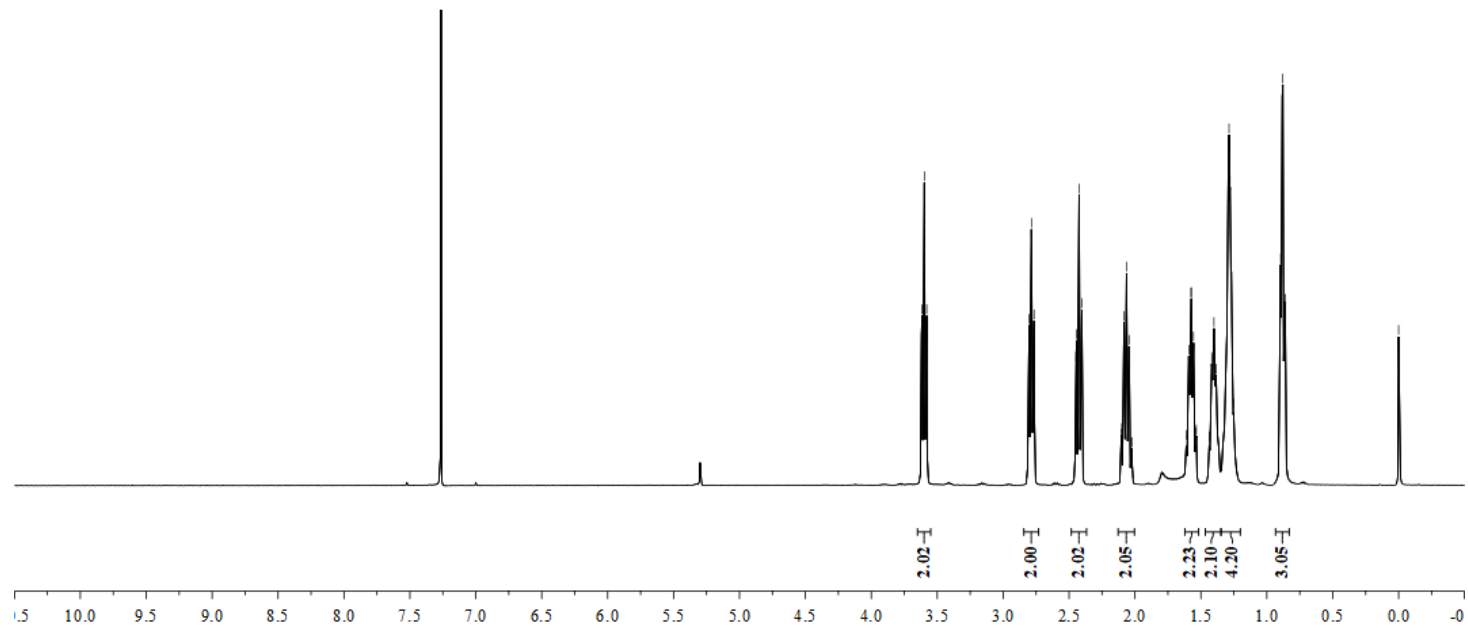

${ }^{13} \mathrm{C}$ NMR (101 MHz, $\left.\mathrm{CDCl}_{3}\right)$ spectrum of compound 2c

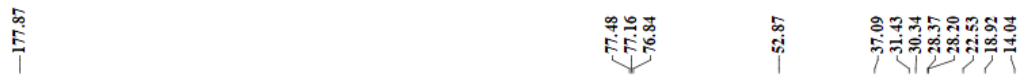

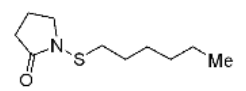

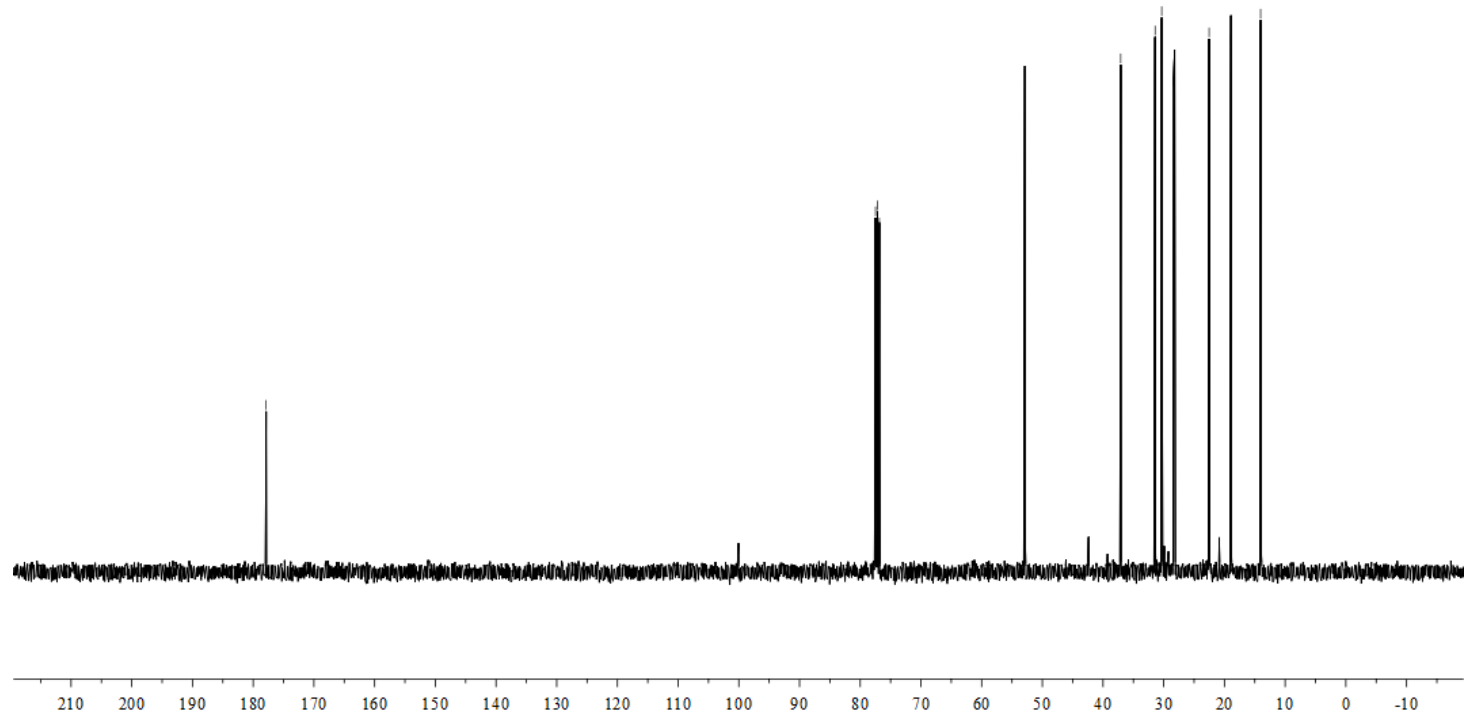


${ }^{1} \mathrm{H}$ NMR (400 MHz, $\mathrm{CDCl}_{3}$ ) spectrum of compound 2d

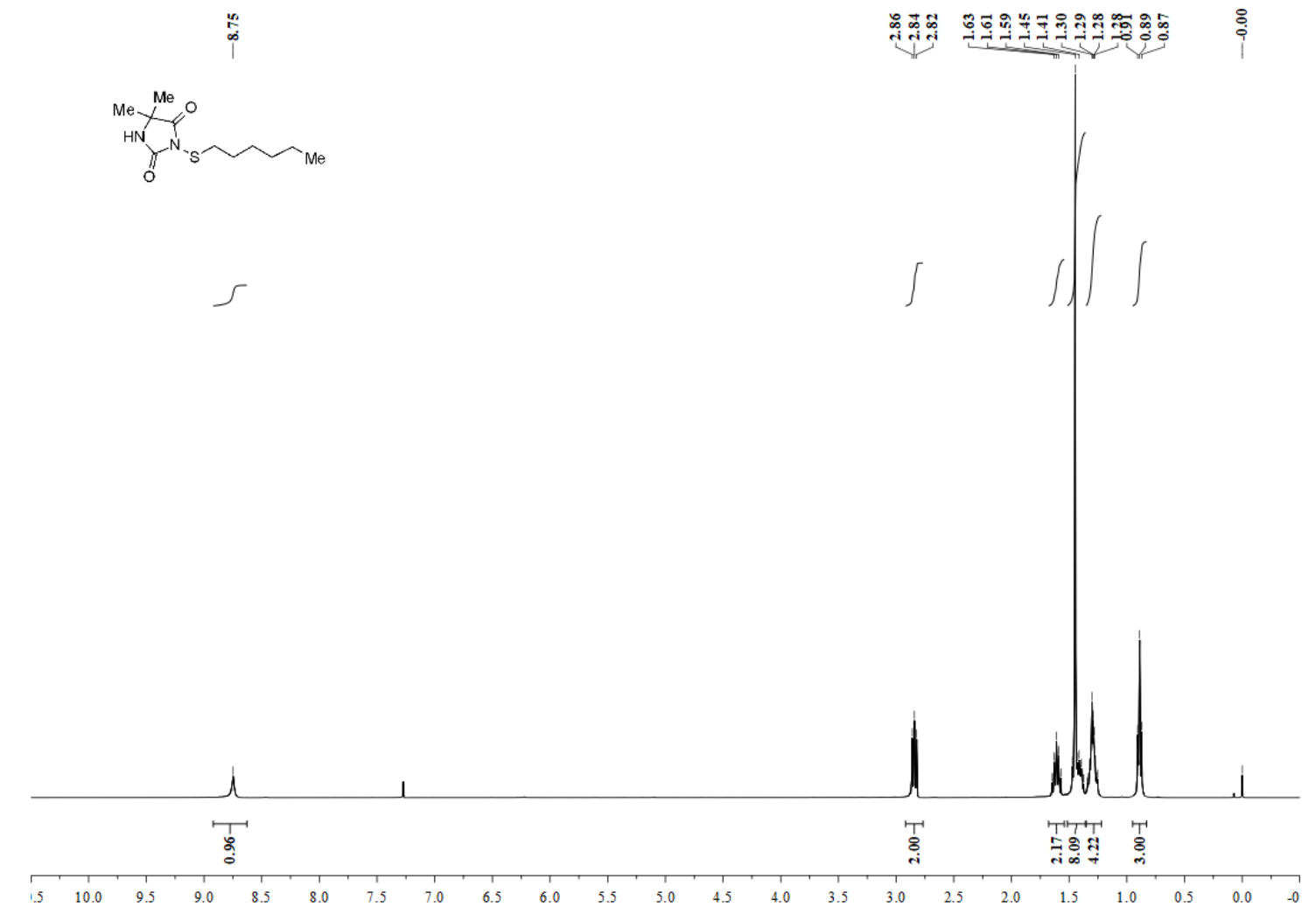

${ }^{13} \mathrm{C}$ NMR (101 MHz, $\left.\mathrm{CDCl}_{3}\right)$ spectrum of compound 2d

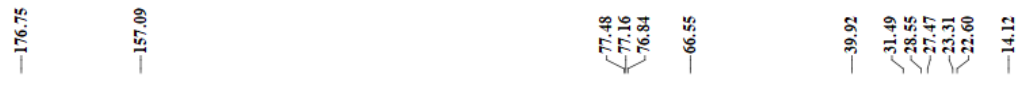

$$
\begin{aligned}
& \underbrace{\text { Me }}_{\text {Me }}
\end{aligned}
$$

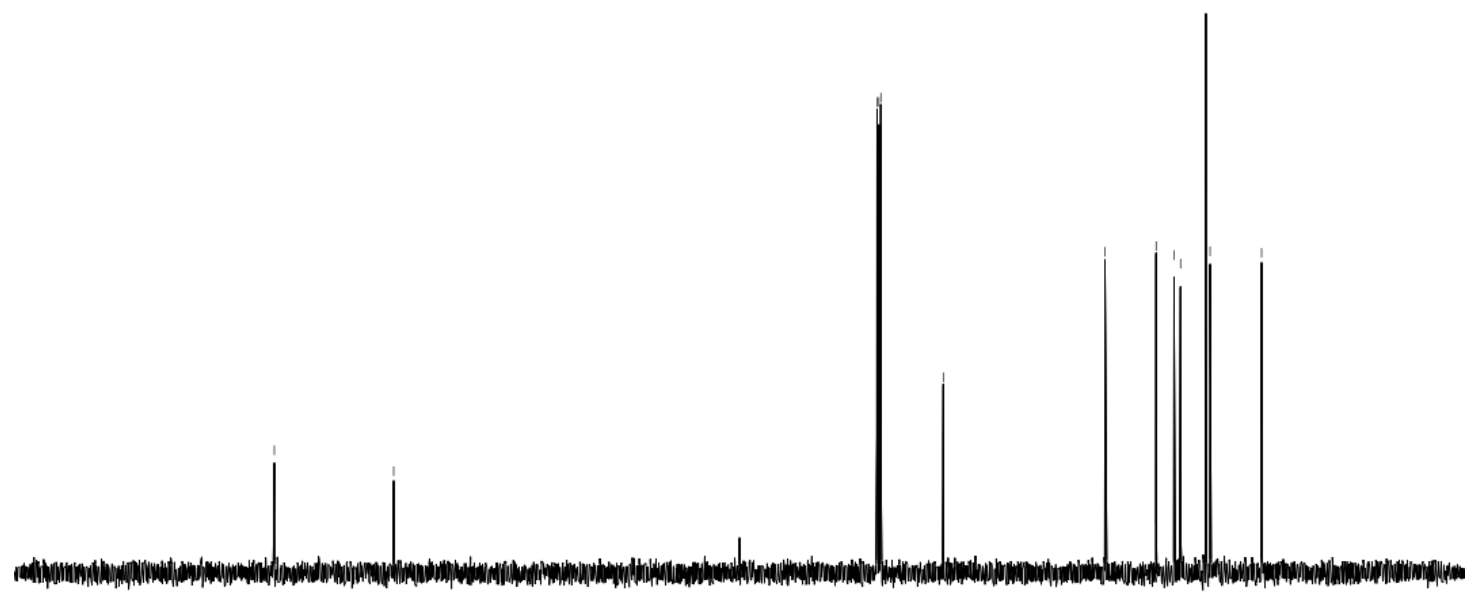


${ }^{1} \mathrm{H}$ NMR (400 MHz, $\left.\mathrm{CDCl}_{3}\right)$ spectrum of compound $\mathbf{2 e}$

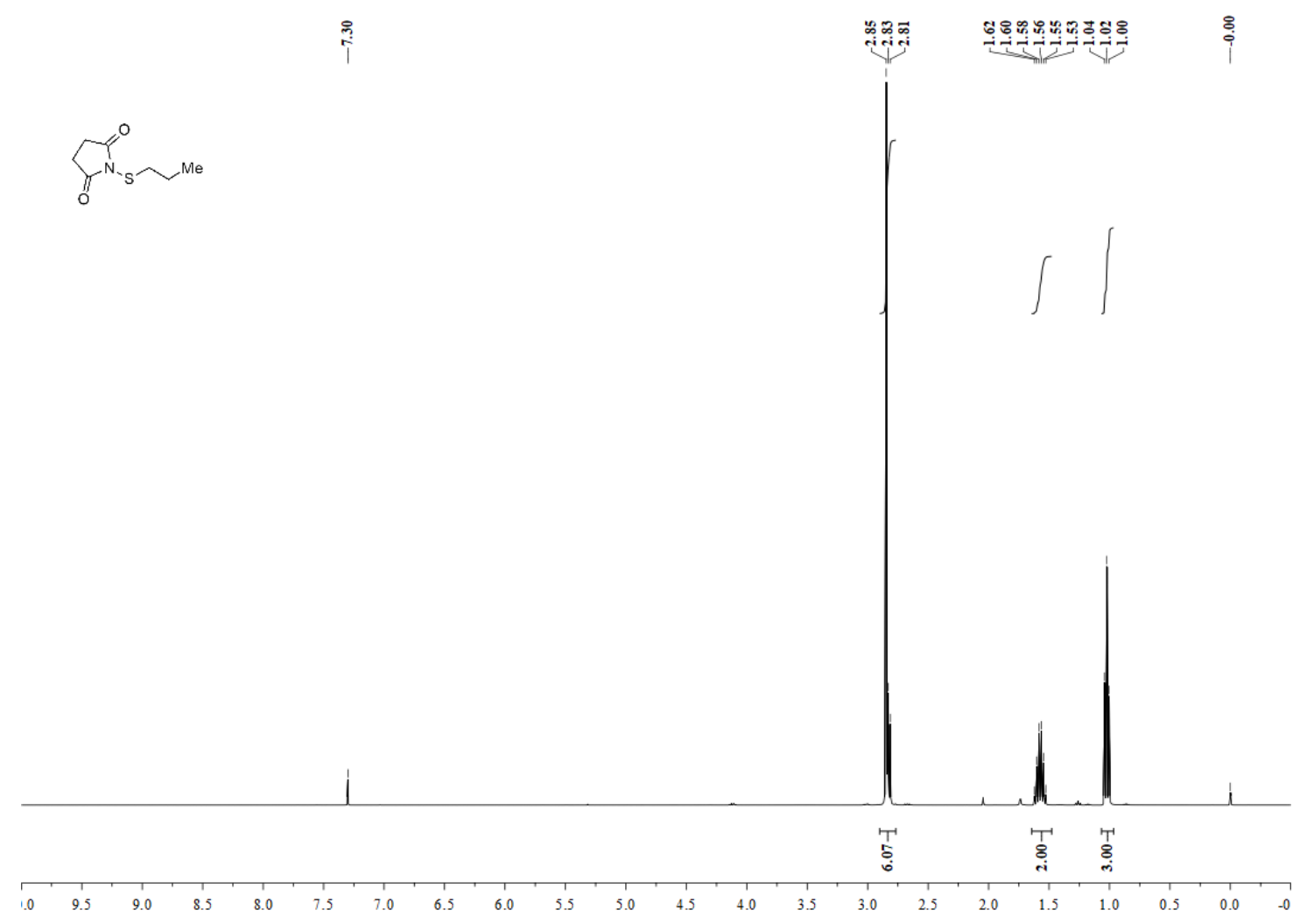

${ }^{13} \mathrm{C}$ NMR $\left(101 \mathrm{MHz}, \mathrm{CDCl}_{3}\right)$ spectrum of compound $\mathbf{2 e}$
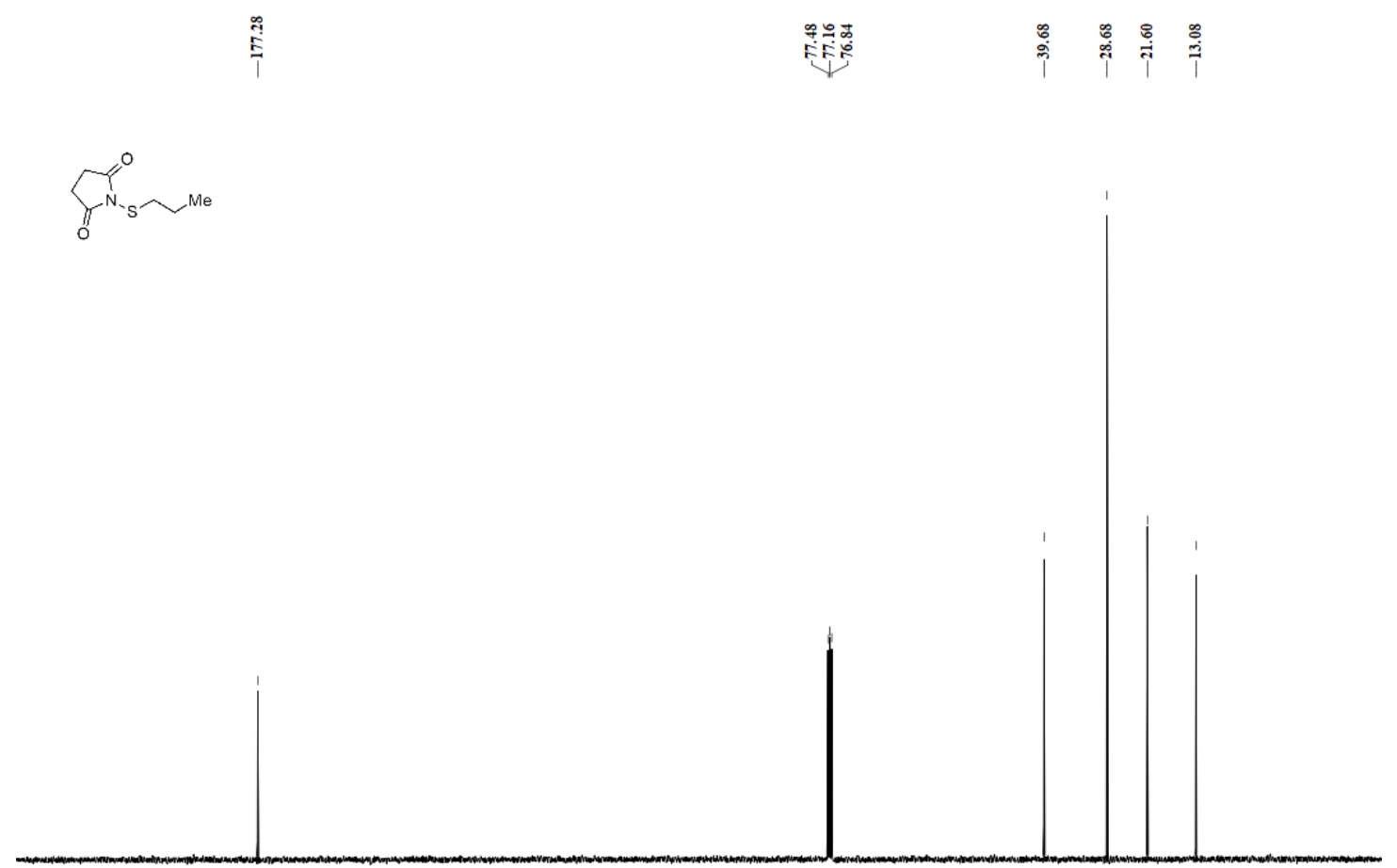

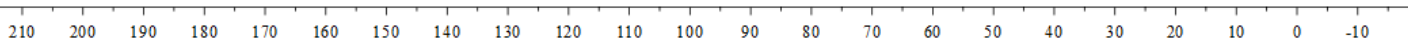


${ }^{1} \mathrm{H}$ NMR (400 MHz, $\mathrm{CDCl}_{3}$ ) spectrum of compound $\mathbf{2 f}$

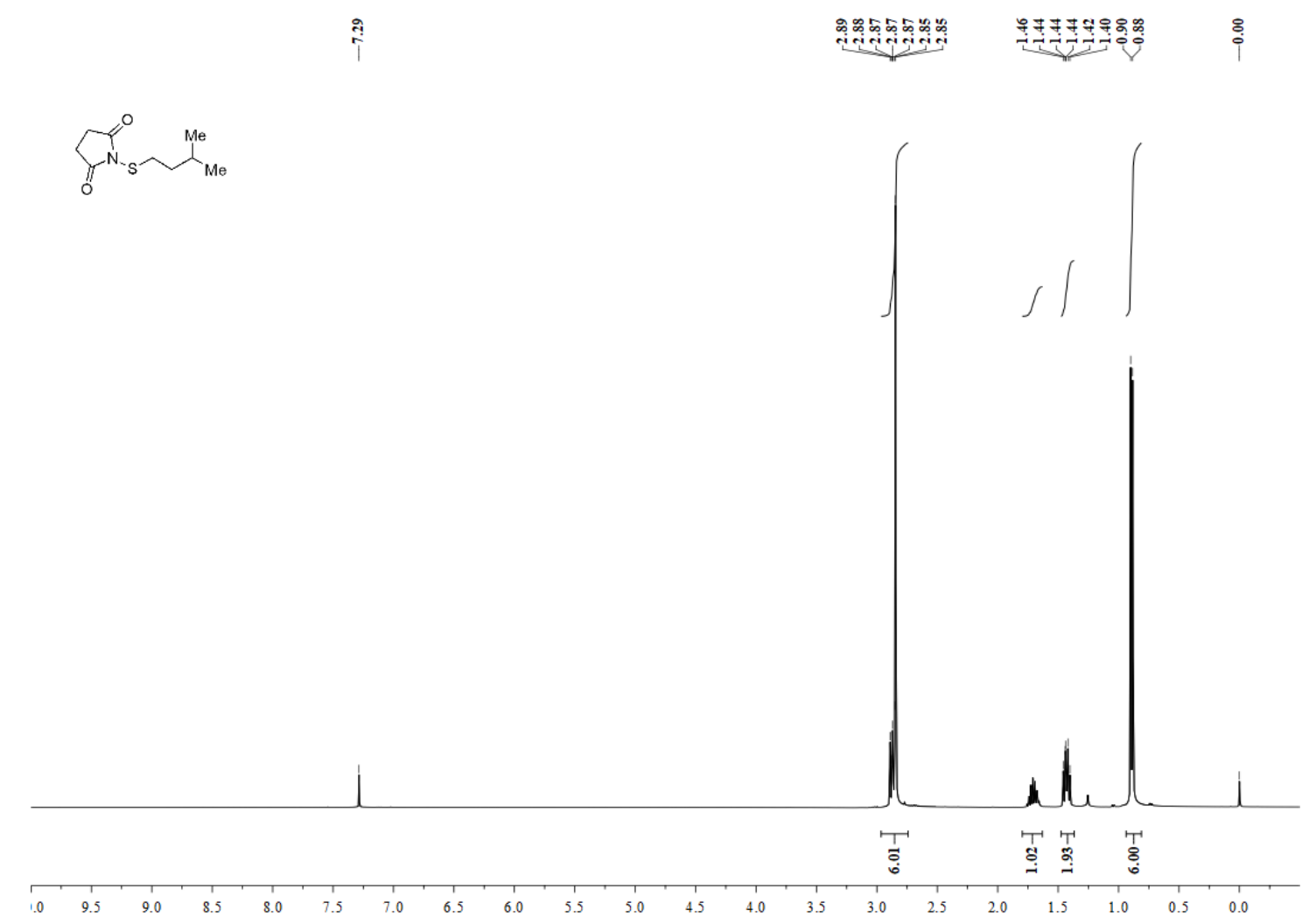

${ }^{13} \mathrm{C}$ NMR $\left(101 \mathrm{MHz}, \mathrm{CDCl}_{3}\right)$ spectrum of compound $\mathbf{2 f}$

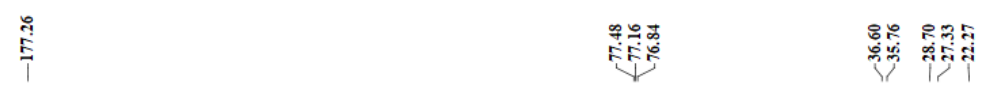

$\overbrace{0}^{N_{N}} \sim_{M e}^{M e}$

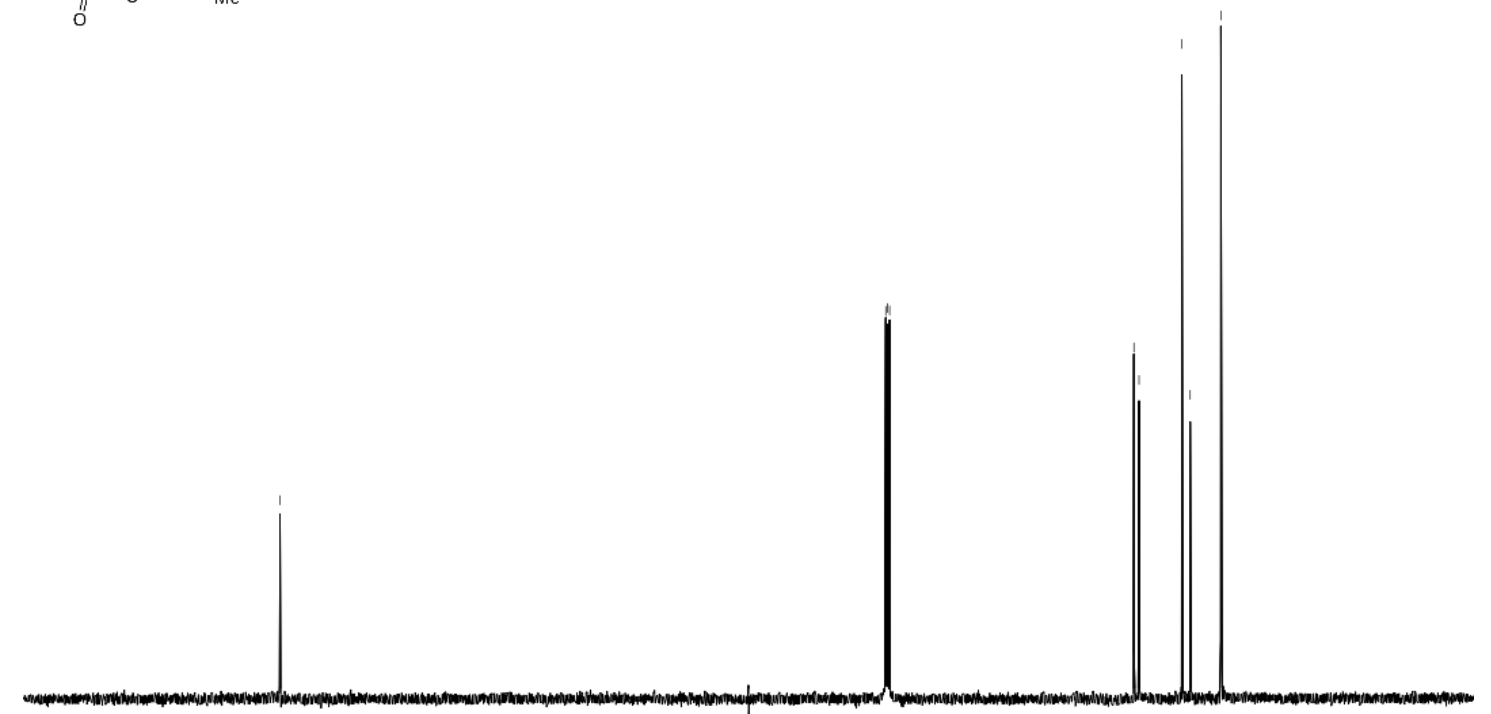


${ }^{1} \mathrm{H}$ NMR (400 MHz, $\mathrm{CDCl}_{3}$ ) spectrum of compound $\mathbf{2 g}$

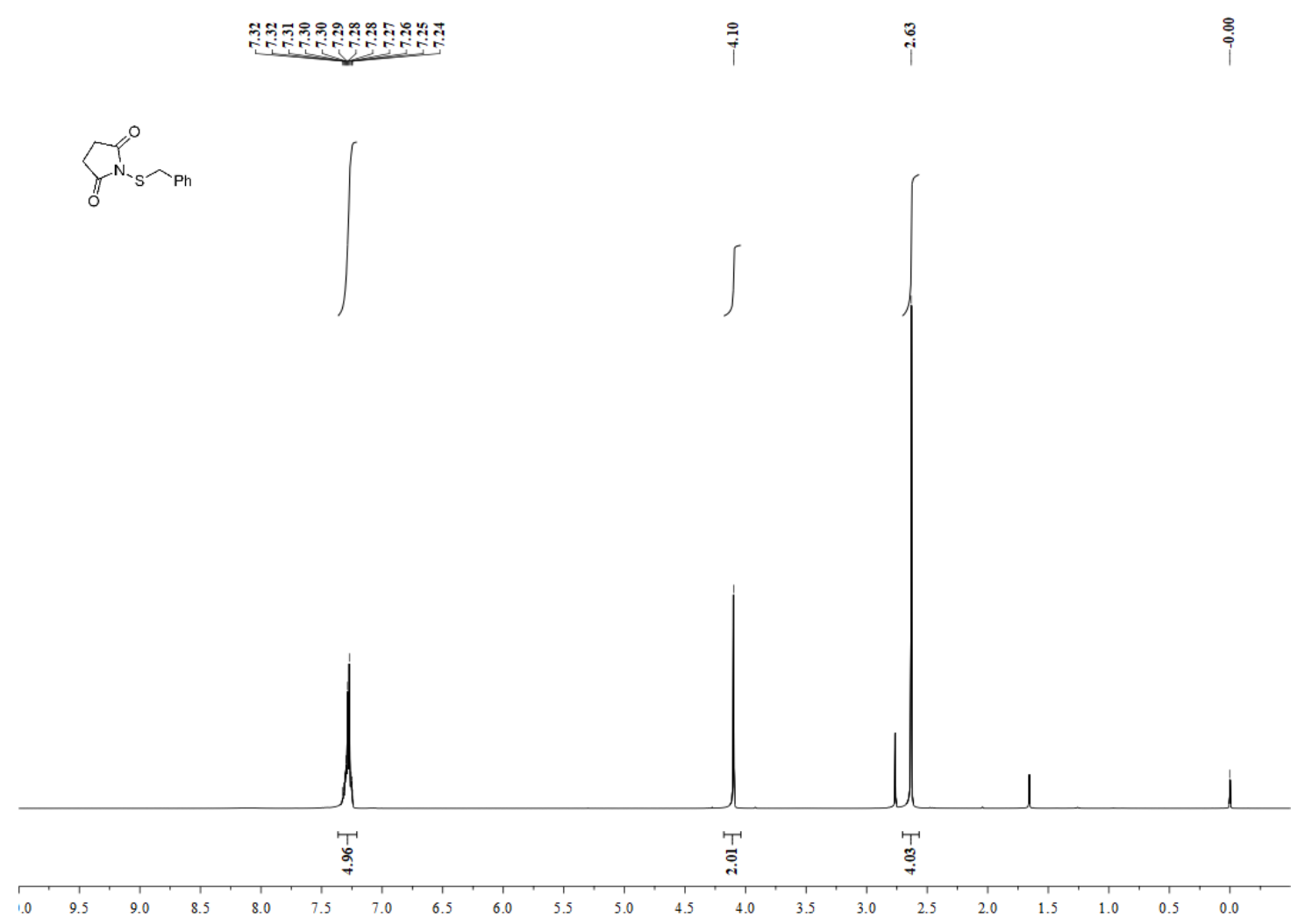

${ }^{13} \mathrm{C}$ NMR (101 MHz, $\left.\mathrm{CDCl}_{3}\right)$ spectrum of compound $\mathbf{2 g}$

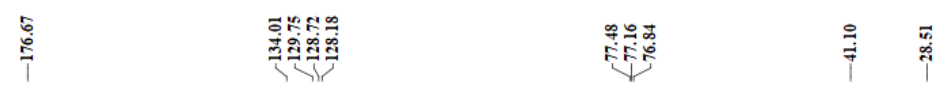

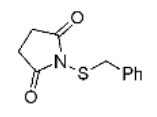

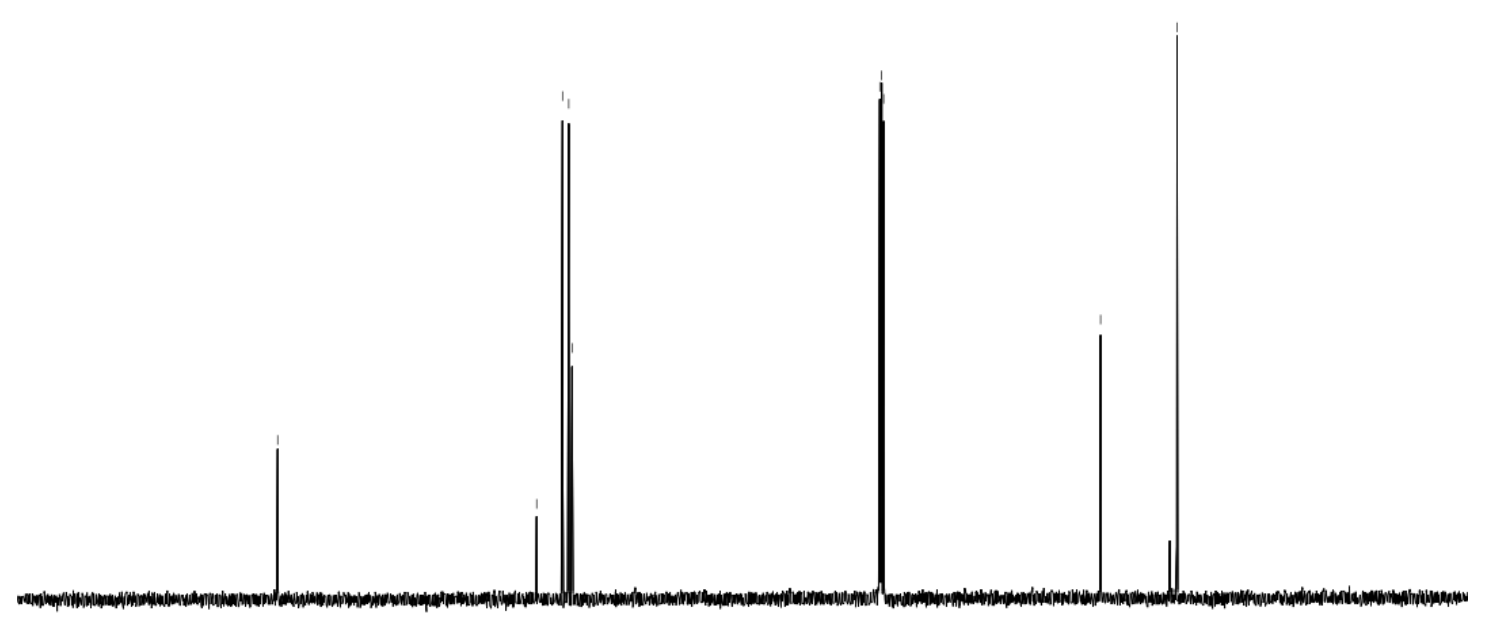

$\begin{array}{llllllllllllllllllllllllll}210 & 200 & 190 & 180 & 170 & 160 & 150 & 140 & 130 & 120 & 110 & 100 & 90 & 80 & 70 & 60 & 50 & 40 & 30 & 20 & 10 & 0 & -10 & 10\end{array}$ 
${ }^{1} \mathrm{H}$ NMR (400 MHz, $\mathrm{CDCl}_{3}$ ) spectrum of compound $\mathbf{2 h}$

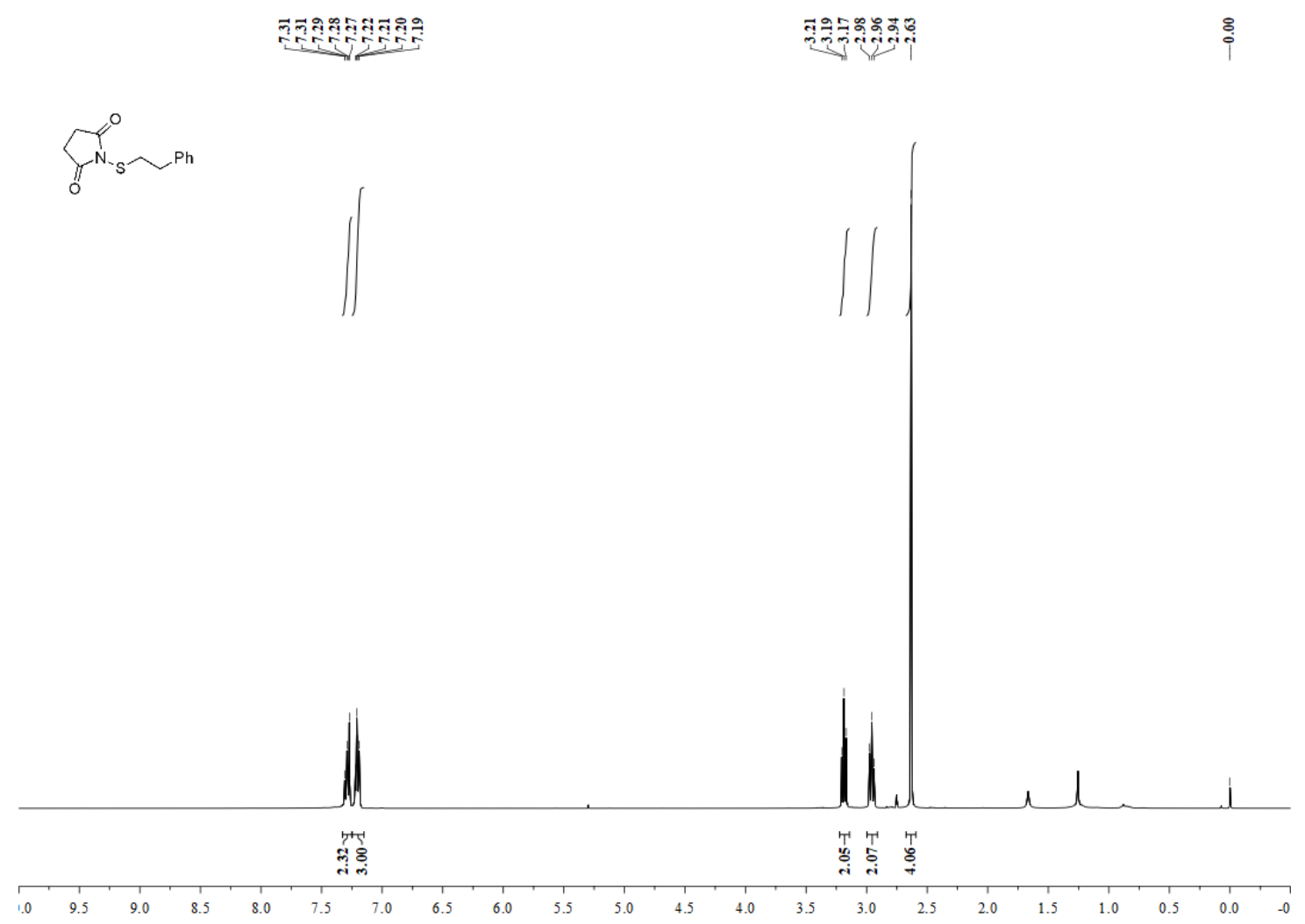

${ }^{13} \mathrm{C}$ NMR (101 MHz, $\mathrm{CDCl}_{3}$ ) spectrum of compound $\mathbf{2 h}$

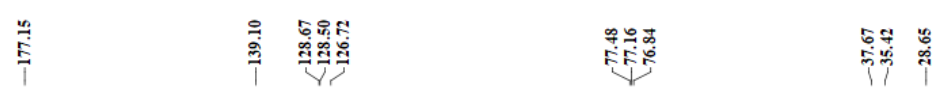

$\overbrace{0}^{N^{N} \cdot S} \sim^{P h}$

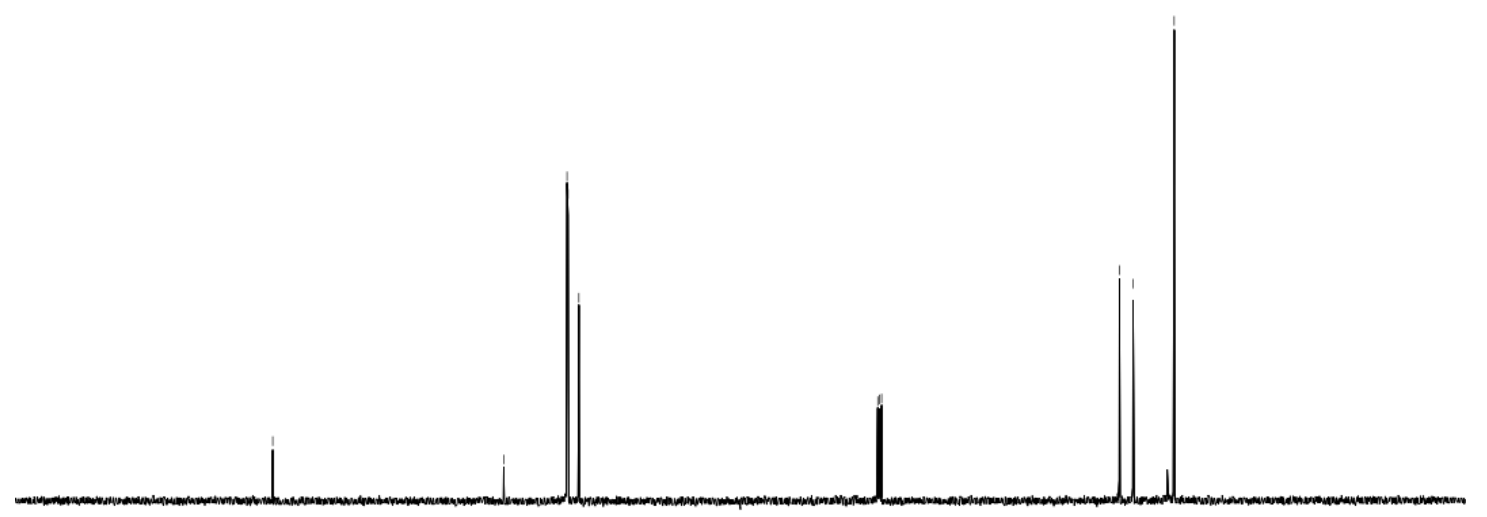


${ }^{1} \mathrm{H} \mathrm{NMR}\left(400 \mathrm{MHz}, \mathrm{CDCl}_{3}\right.$ ) spectrum of compound $\mathbf{2 i}$

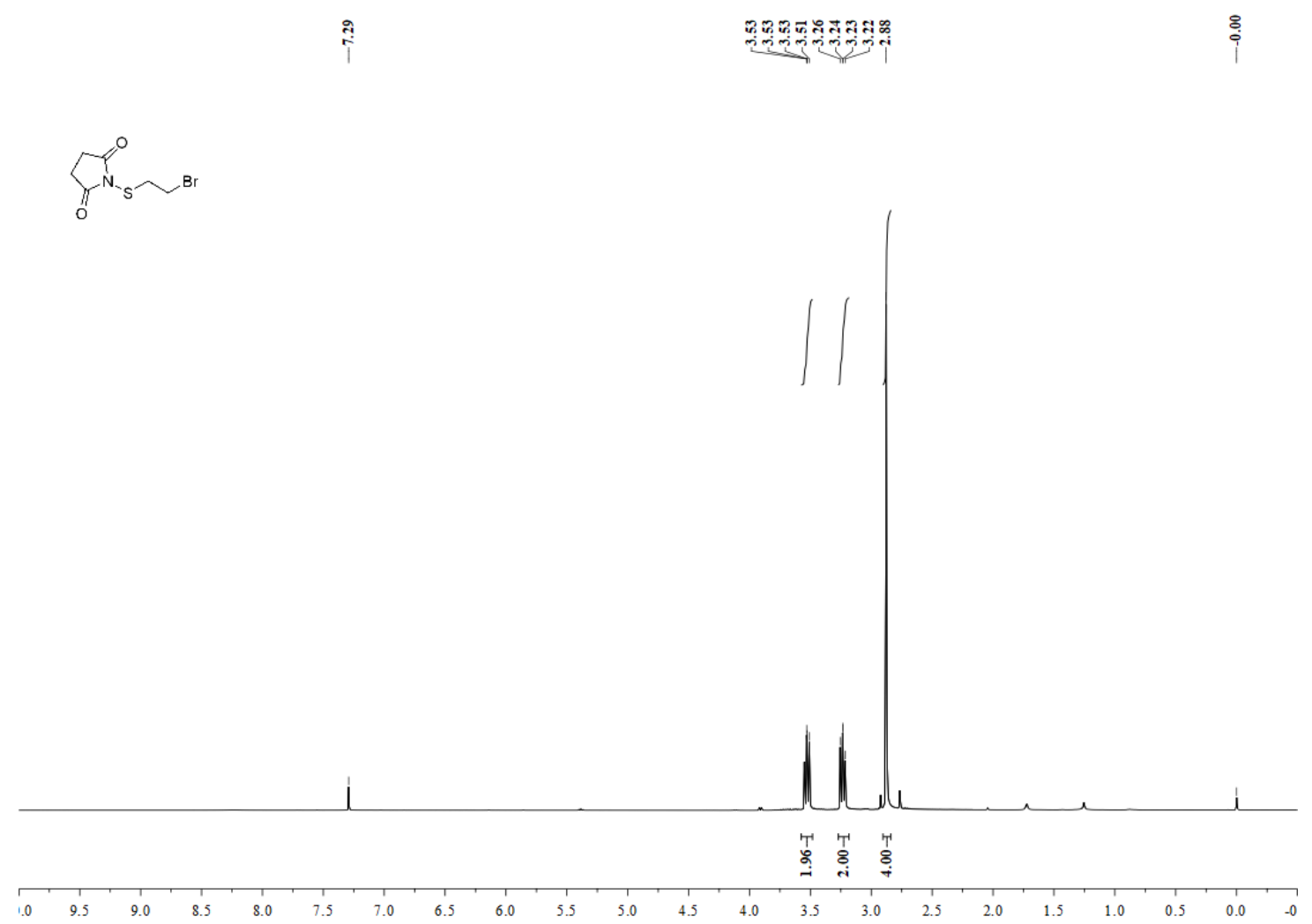

${ }^{13} \mathrm{C}$ NMR $\left(101 \mathrm{MHz}, \mathrm{CDCl}_{3}\right)$ spectrum of compound $\mathbf{2 i}$
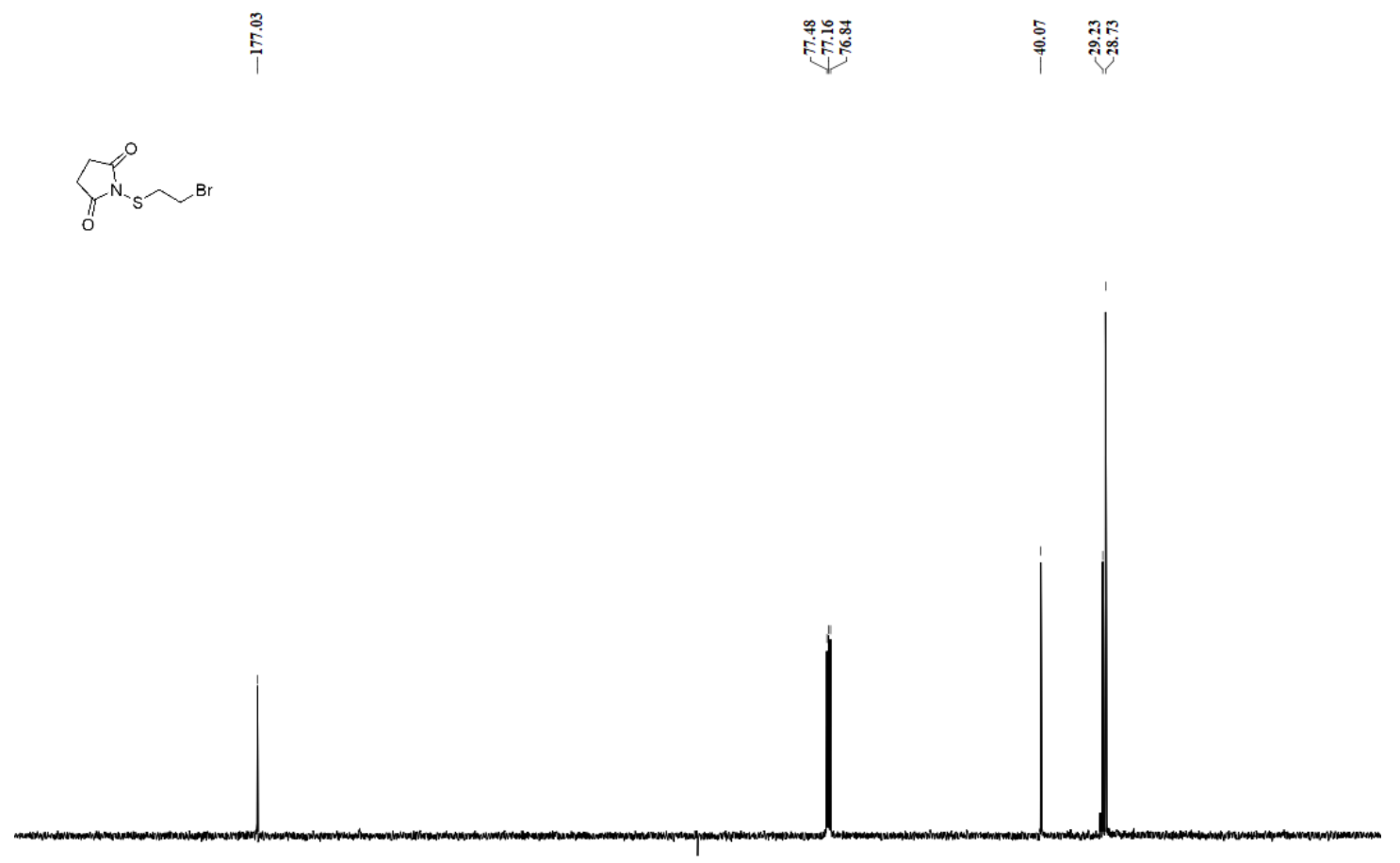
${ }^{1} \mathrm{H}$ NMR $\left(400 \mathrm{MHz}, \mathrm{CDCl}_{3}\right)$ spectrum of compound $\mathbf{2} \mathbf{j}$

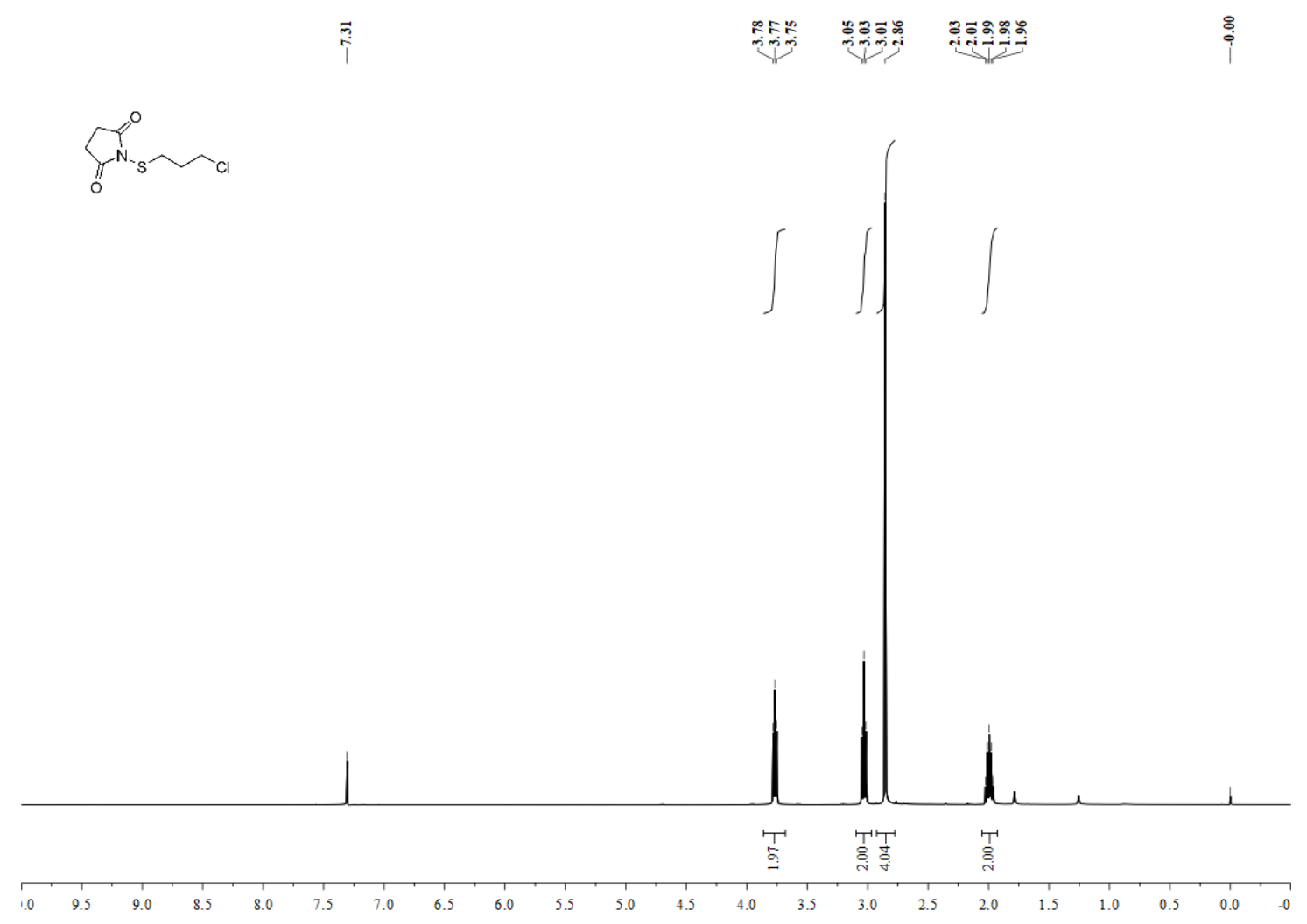

${ }^{13} \mathrm{C}$ NMR $\left(101 \mathrm{MHz}, \mathrm{CDCl}_{3}\right)$ spectrum of compound $\mathbf{2 j}$

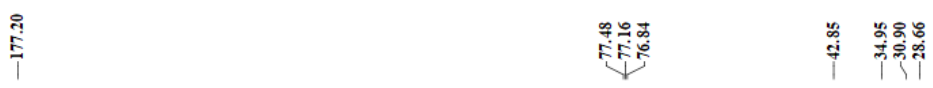

$\mathrm{H}_{0}^{\mathrm{N}} \mathrm{N}^{\mathrm{S}} \mathrm{MCl}_{\mathrm{Cl}}$

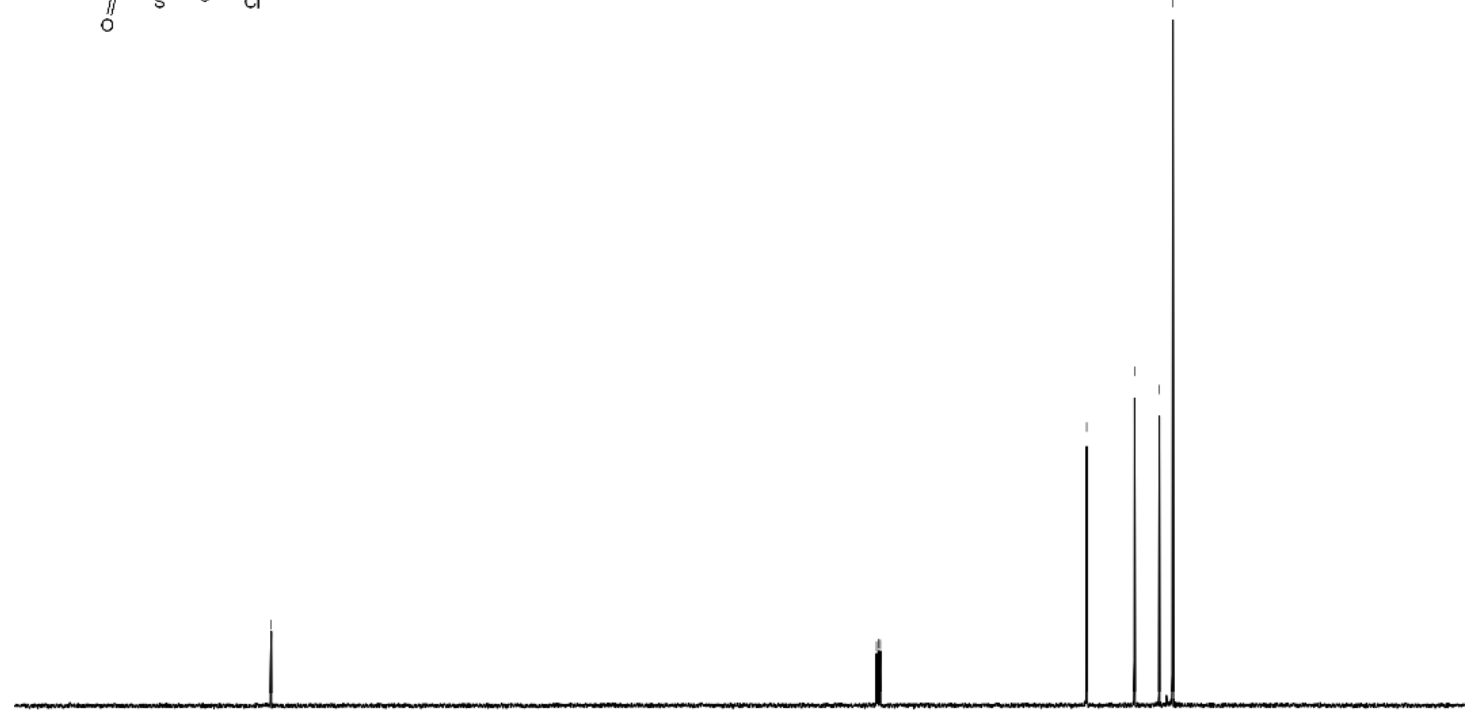

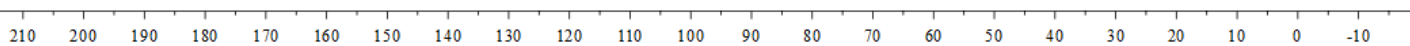


${ }^{1} \mathrm{H}$ NMR (400 MHz, $\mathrm{CDCl}_{3}$ ) spectrum of compound $\mathbf{2 k}$

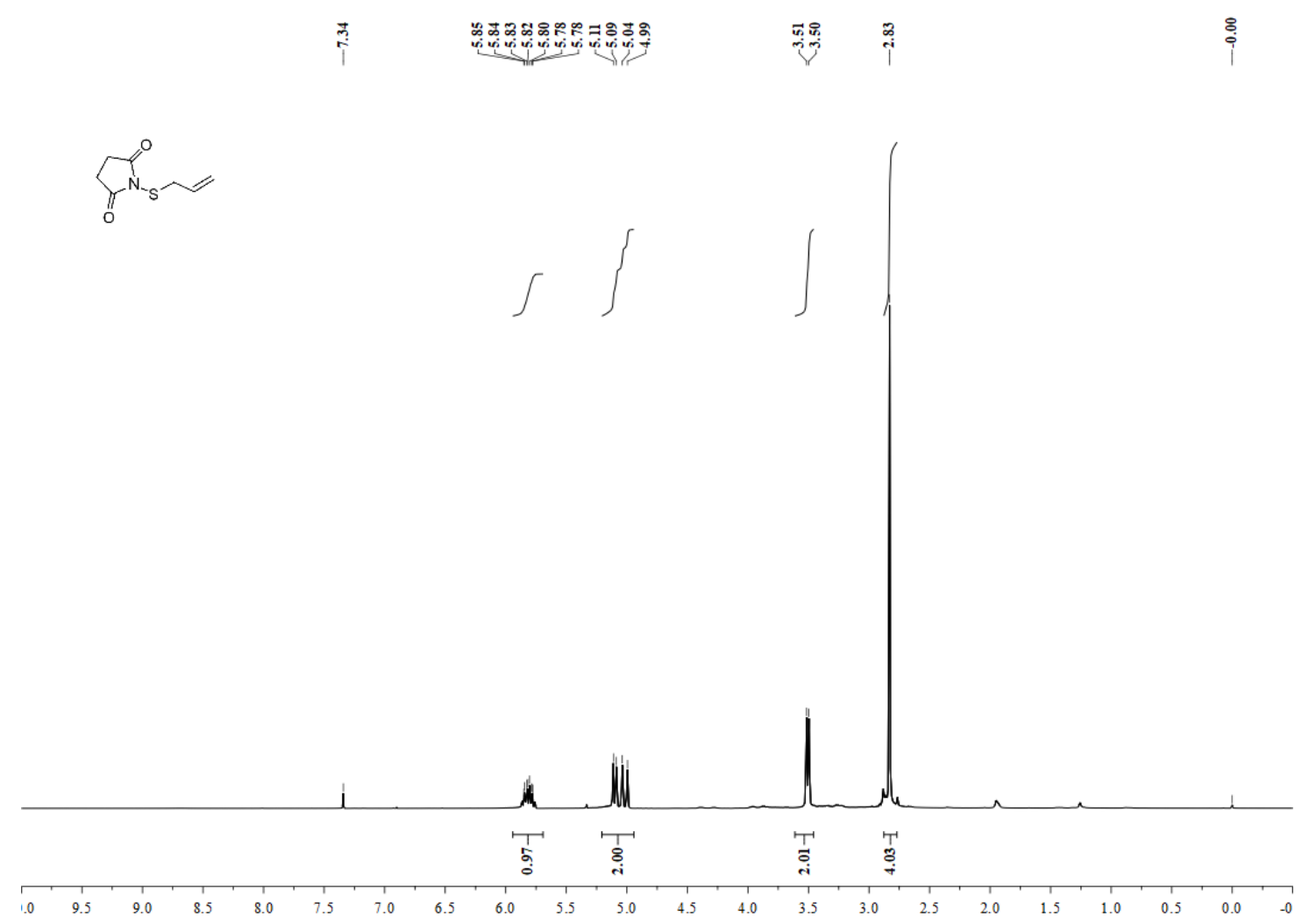

${ }^{13} \mathrm{C}$ NMR (101 MHz, $\left.\mathrm{CDCl}_{3}\right)$ spectrum of compound $\mathbf{2 k}$

总

$\sum_{0}^{\infty}$

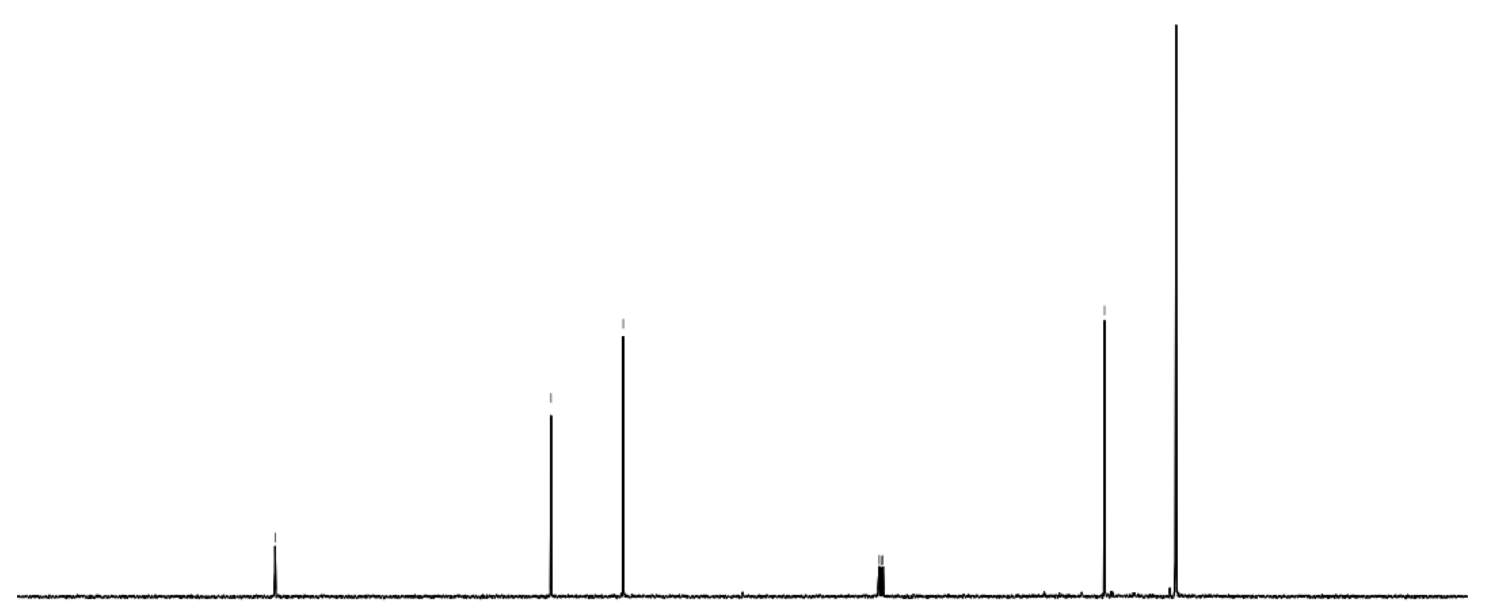

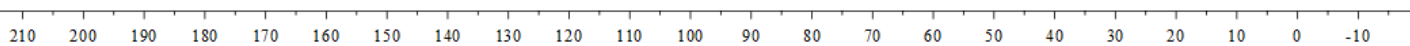


${ }^{1} \mathrm{H} \mathrm{NMR}\left(400 \mathrm{MHz}, \mathrm{CDCl}_{3}\right.$ ) spectrum of compound $\mathbf{2 l}$

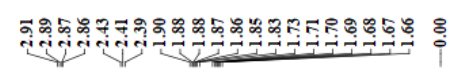

$\overbrace{0}^{\gamma^{N}} \sim^{\mathrm{C}} \sim^{\mathrm{CN}}$

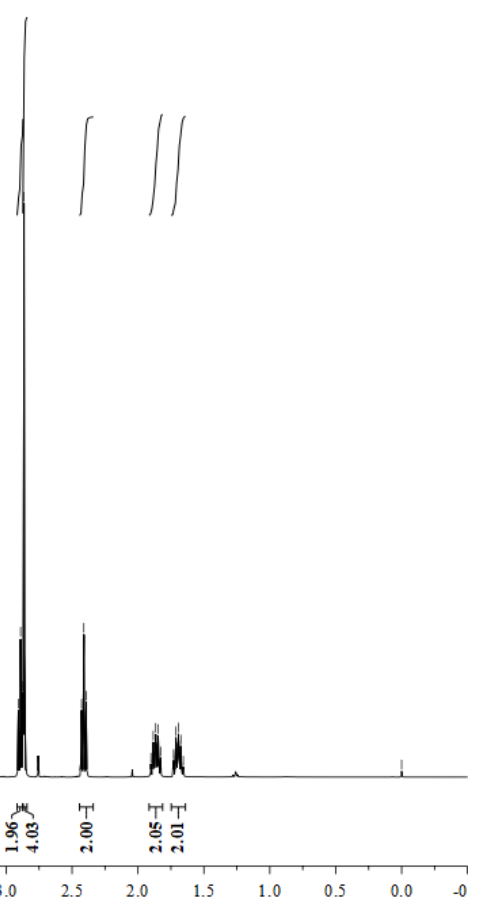

${ }^{13} \mathrm{C}$ NMR (101 MHz, $\left.\mathrm{CDCl}_{3}\right)$ spectrum of compound 2l

$\stackrel{\mathscr{2}}{\mathrm{I}}$

$\stackrel{2}{\stackrel{2}{1}}$

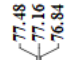

कृ

$\overbrace{0}^{N^{N}} \sim^{C N}$

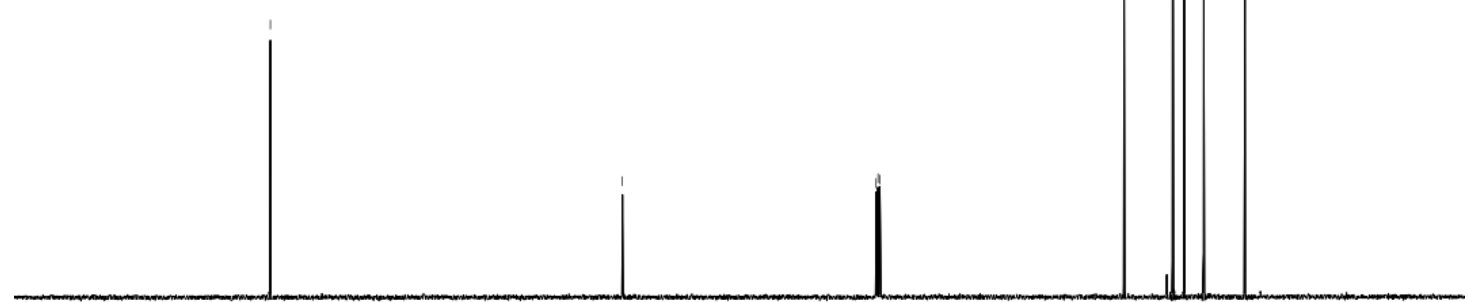

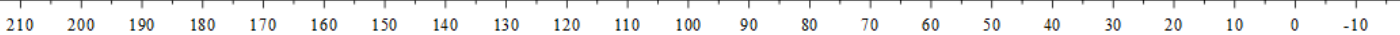


${ }^{1} \mathrm{H}$ NMR (400 MHz, $\mathrm{CDCl}_{3}$ ) spectrum of compound $\mathbf{C 2}$

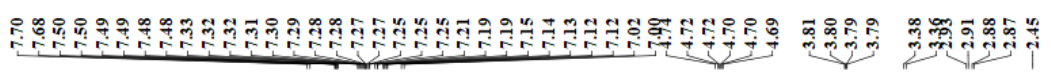

$\stackrel{8}{i}$
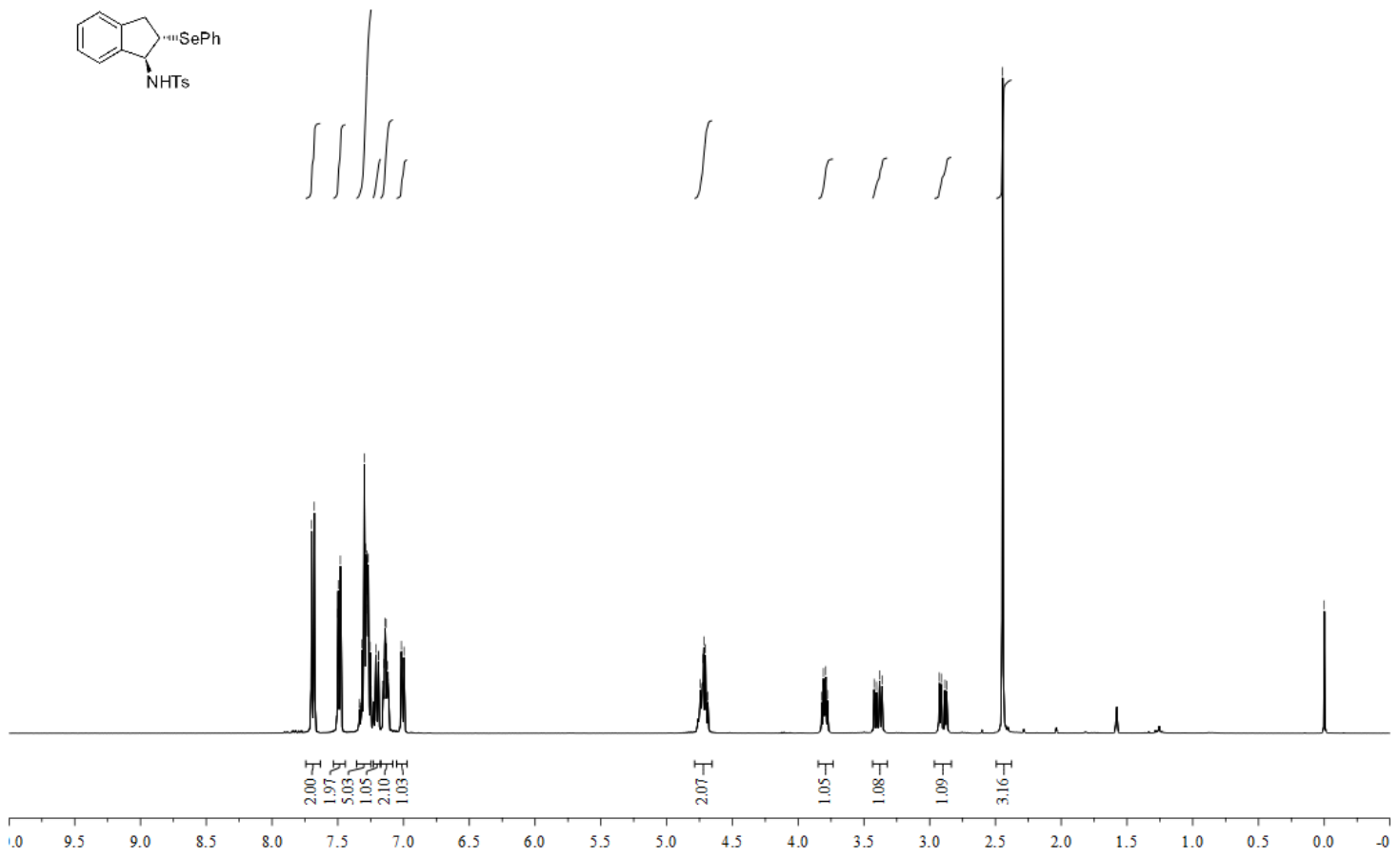

${ }^{13} \mathrm{C}$ NMR (101 MHz, $\mathrm{CDCl}_{3}$ ) spectrum of compound $\mathbf{C 2}$

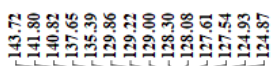

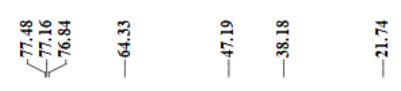
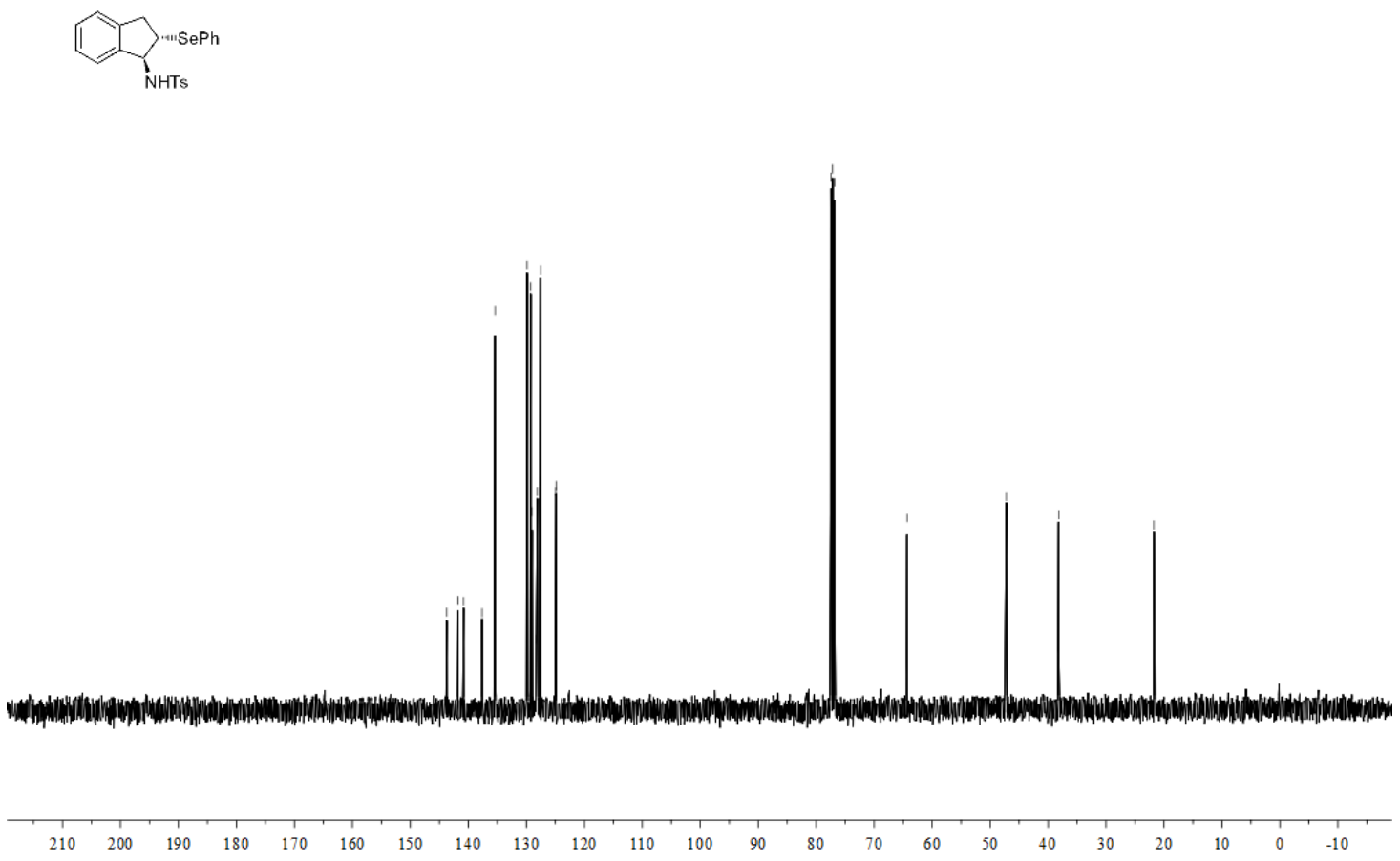

S78 
${ }^{1} \mathrm{H}$ NMR (400 MHz, $\mathrm{CDCl}_{3}$ ) spectrum of compound $\mathbf{C 3}$

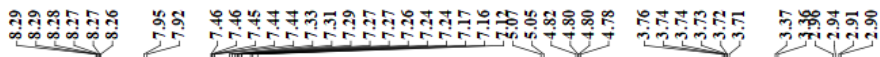<smiles>CC1CC=CC1</smiles><smiles>CC#CCCC</smiles>
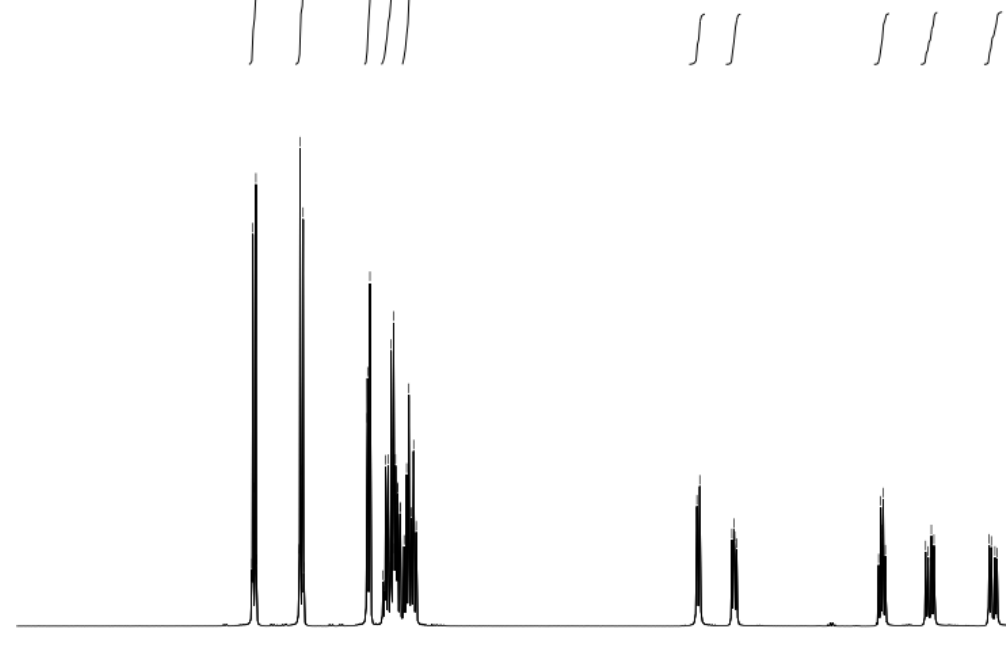

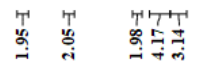

$\stackrel{8}{\rightarrow} \stackrel{8}{-}$

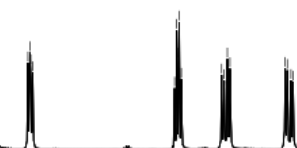

草罗

$\begin{array}{lllllllllllllllllllllllllllll}1.0 & 9.5 & 9.0 & 8.5 & 1.0 & 1.5 & 7.0 & 6.5 & 6.0 & 5.5 & 5.0 & 4.5 & 1.0 & 3.5 & 3.0 & 2.5 & 2.0 & 1.5 & 1.0 & 0.5 & 0.0 & -0\end{array}$

${ }^{13} \mathrm{C}$ NMR (101 MHz, $\left.\mathrm{CDCl}_{3}\right)$ spectrum of compound $\mathbf{C 3}$

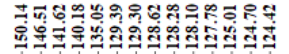

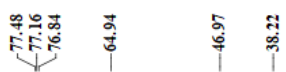<smiles>CC(C)Cc1ccccc1</smiles>

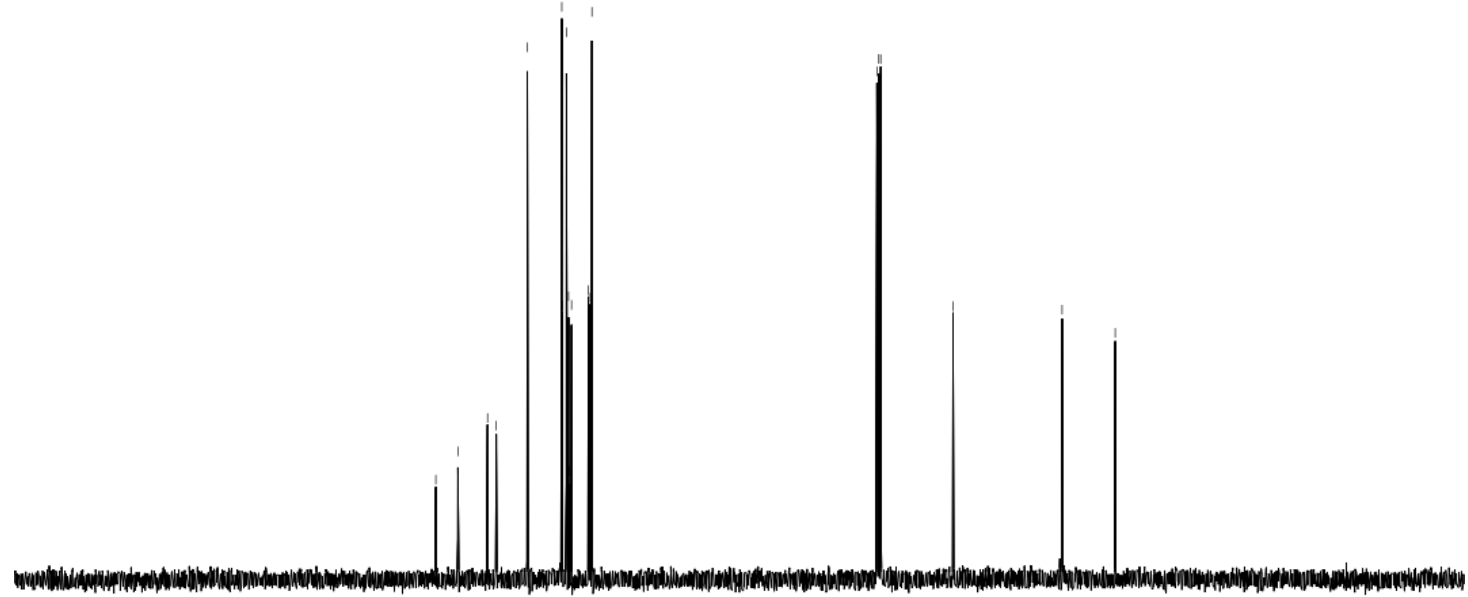


${ }^{1} \mathrm{H}$ NMR (400 MHz, $\mathrm{CDCl}_{3}$ ) spectrum of compound $\mathbf{C 4}$

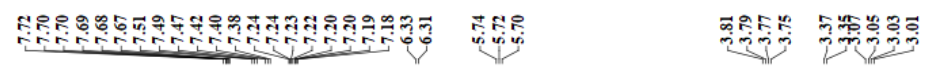

$\stackrel{8}{i}$

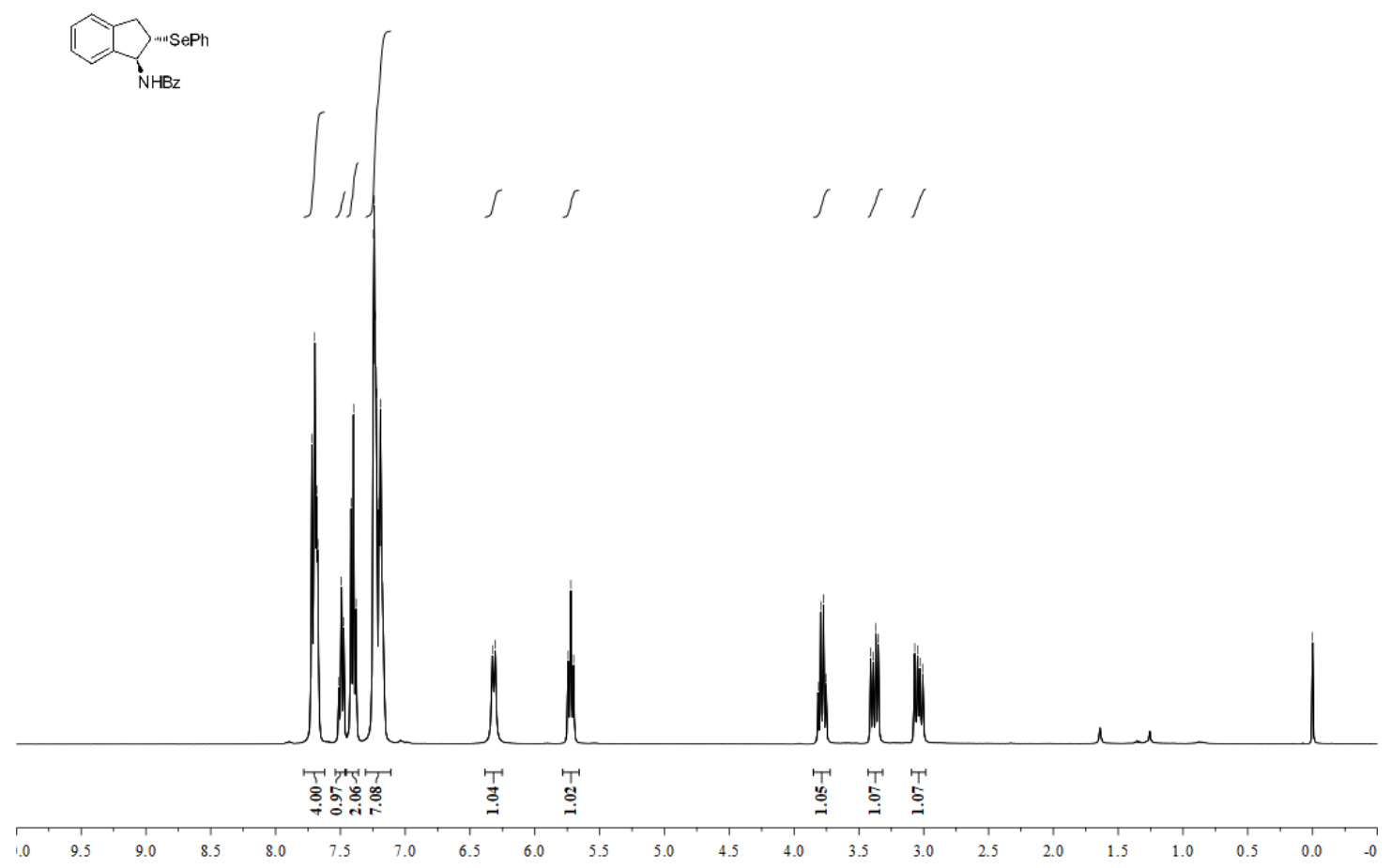

${ }^{13} \mathrm{C}$ NMR (101 MHz, $\left.\mathrm{CDCl}_{3}\right)$ spectrum of compound $\mathbf{C 4}$
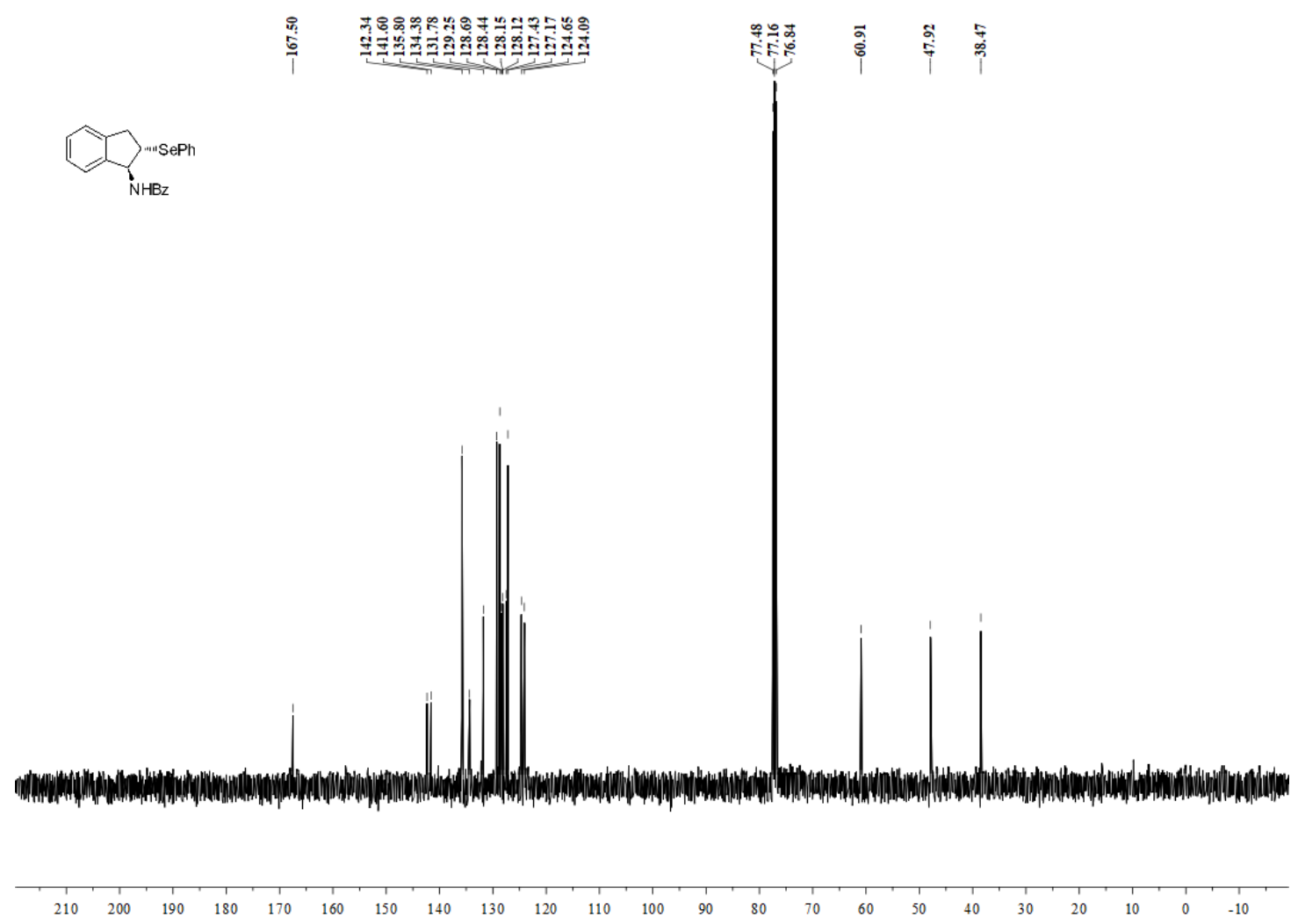

S80 
${ }^{1} \mathrm{H}$ NMR (400 MHz, $\mathrm{CDCl}_{3}$ ) spectrum of compound $\mathbf{C 9}$

ㄴ.

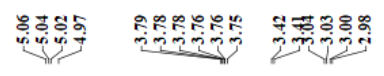

8
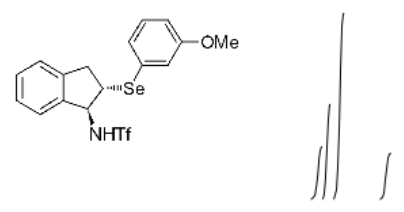

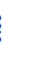

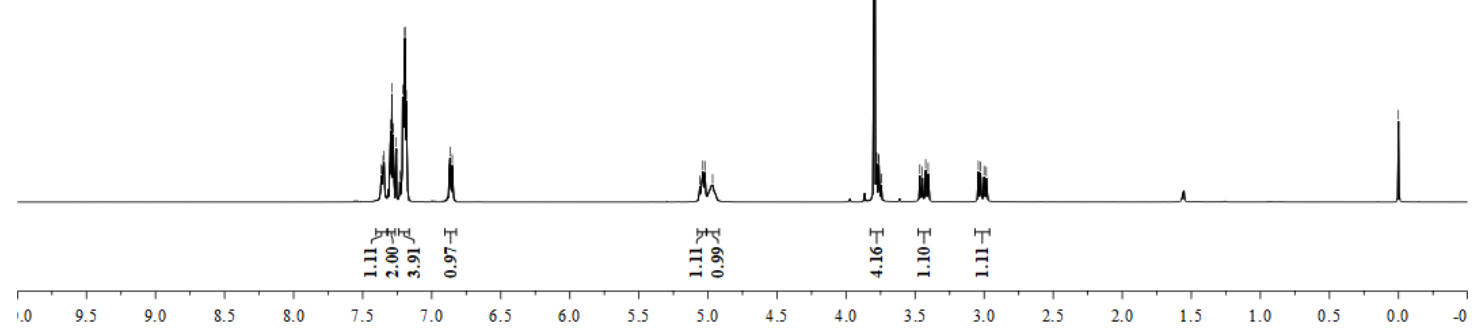

${ }^{13} \mathrm{C}$ NMR (101 MHz, $\mathrm{CDCl}_{3}$ ) spectrum of compound $\mathbf{C 9}$

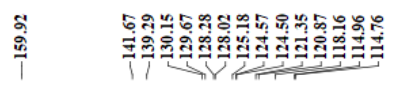

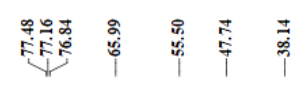
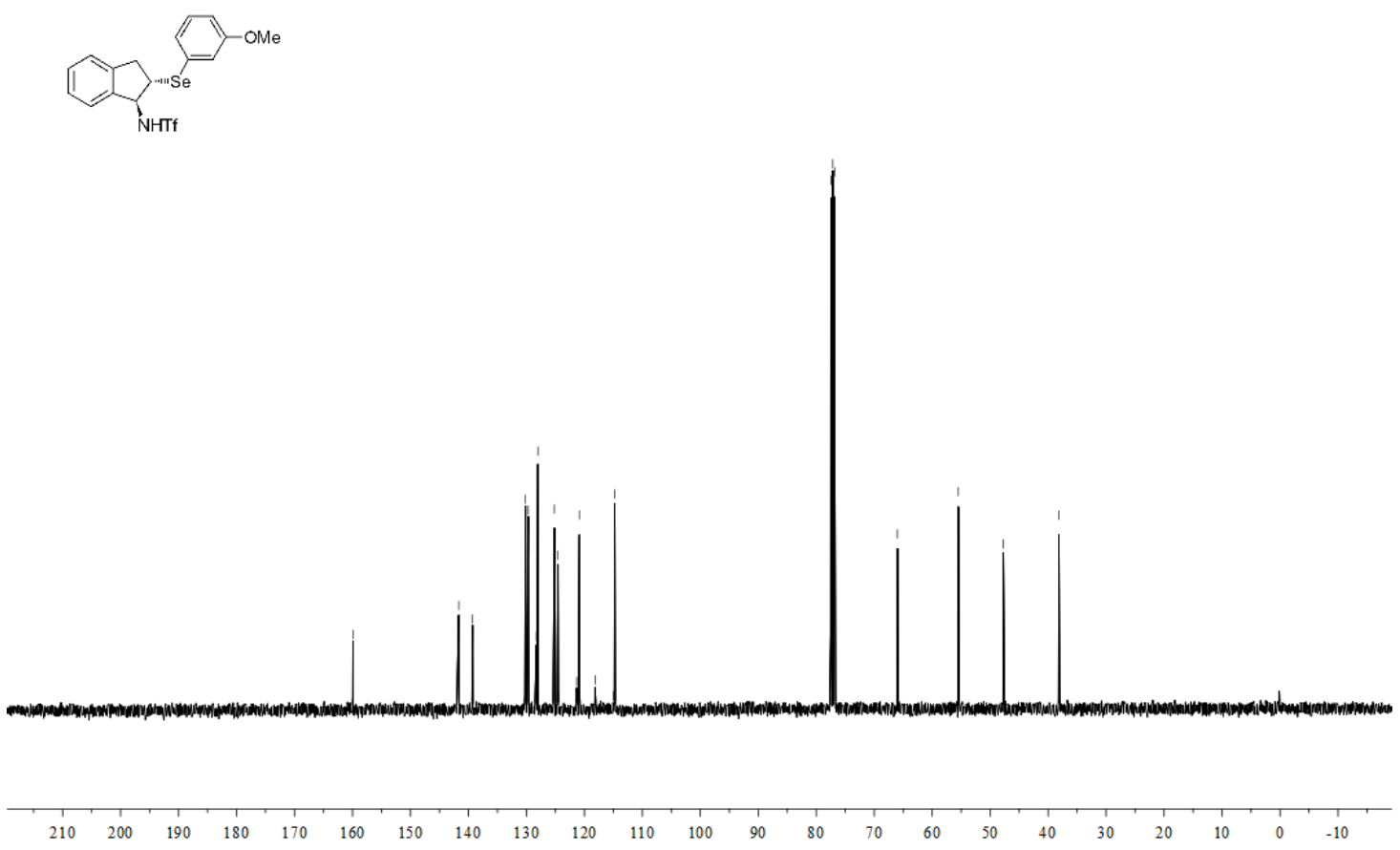

S81 
${ }^{1} \mathrm{H}$ NMR (400 MHz, $\mathrm{CDCl}_{3}$ ) spectrum of compound $\mathbf{C 1 0}$
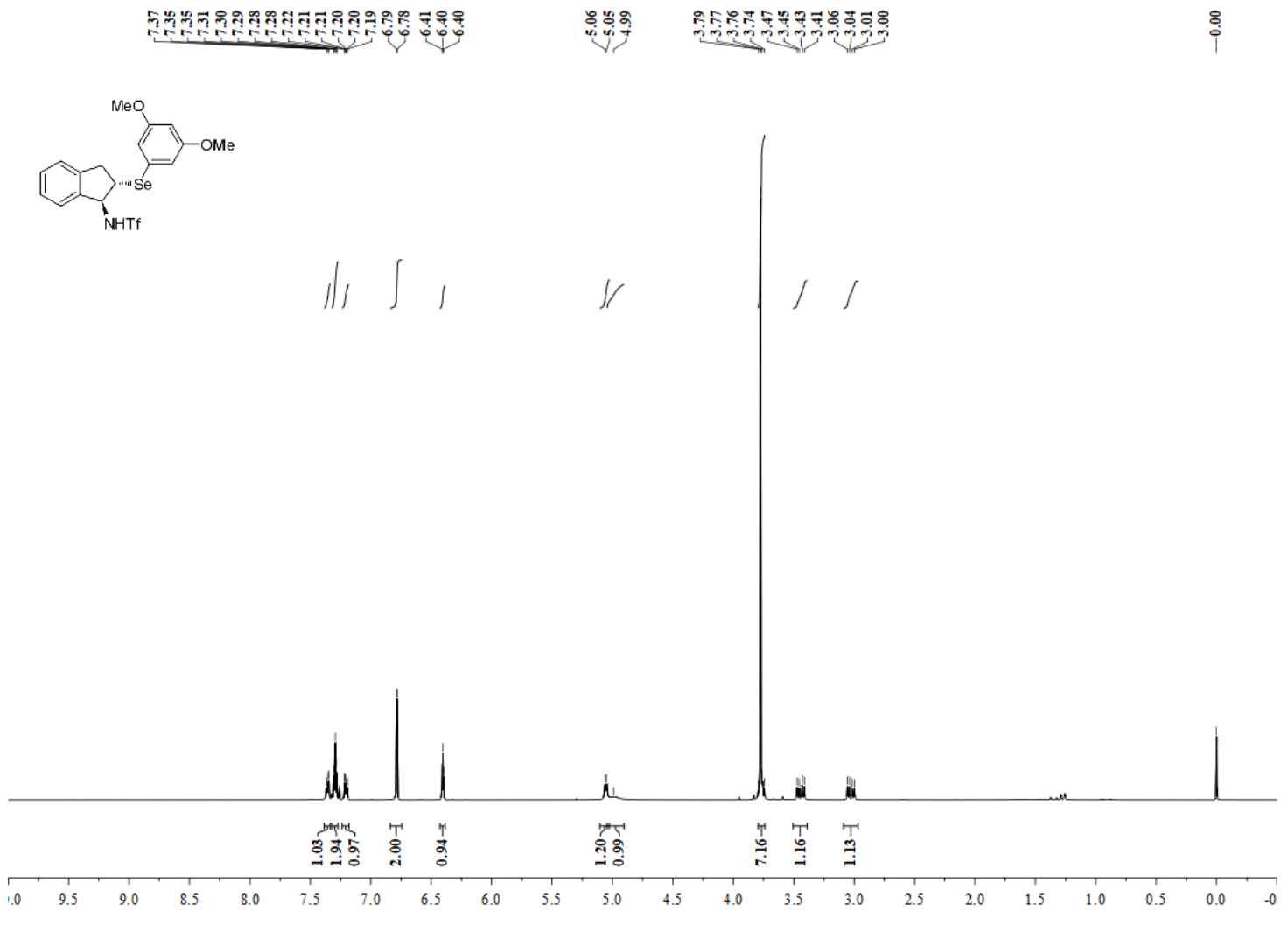

${ }^{13} \mathrm{C}$ NMR (101 MHz, $\mathrm{CDCl}_{3}$ ) spectrum of compound $\mathbf{C 1 0}$ $\stackrel{8}{i}$

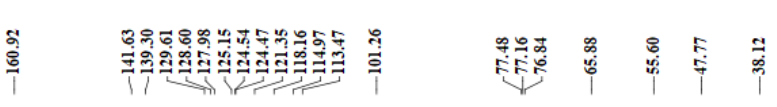
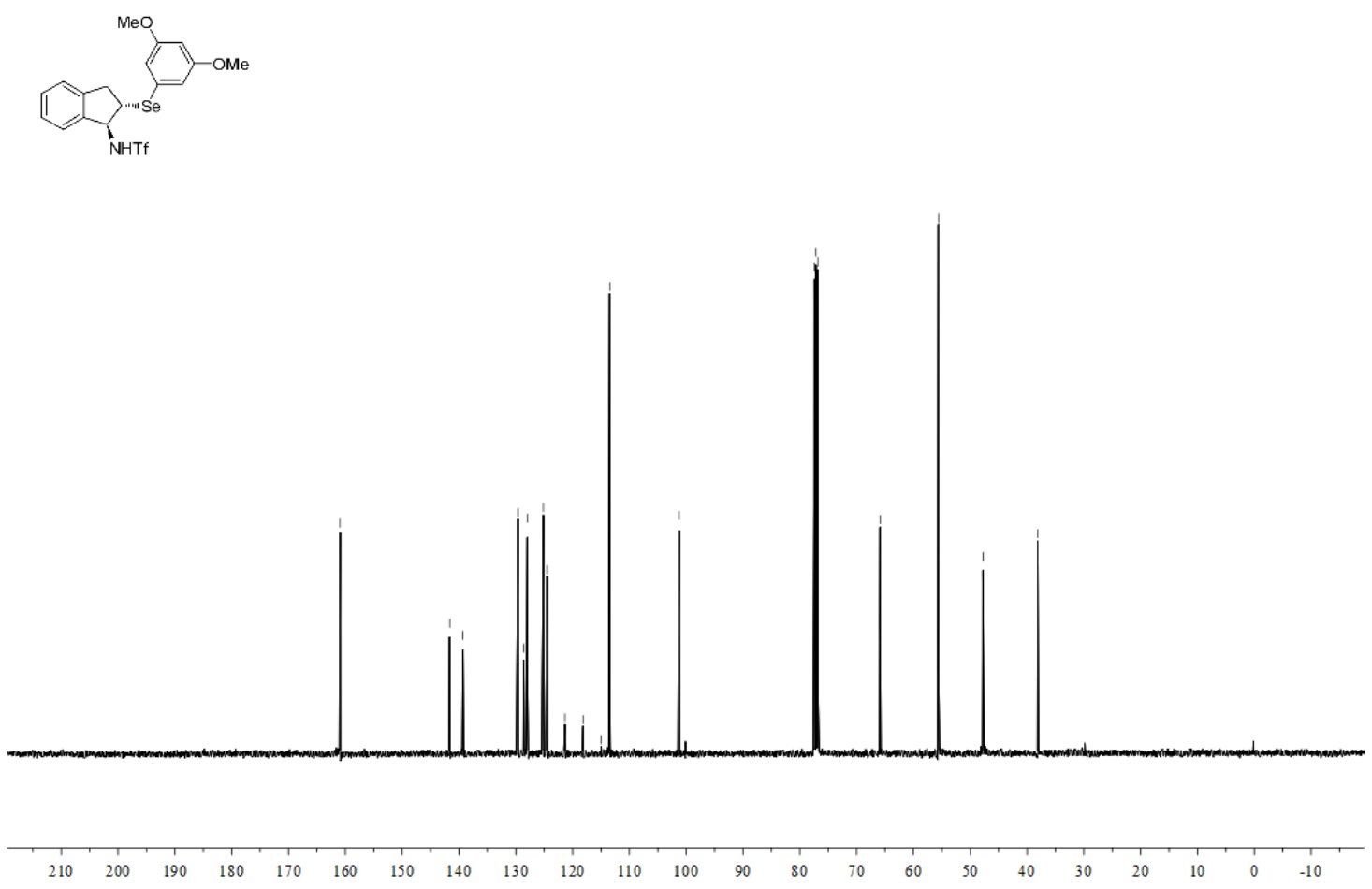

S82 
${ }^{1} \mathrm{H}$ NMR (400 MHz, $\mathrm{CDCl}_{3}$ ) spectrum of compound $\mathbf{C 1 4}$

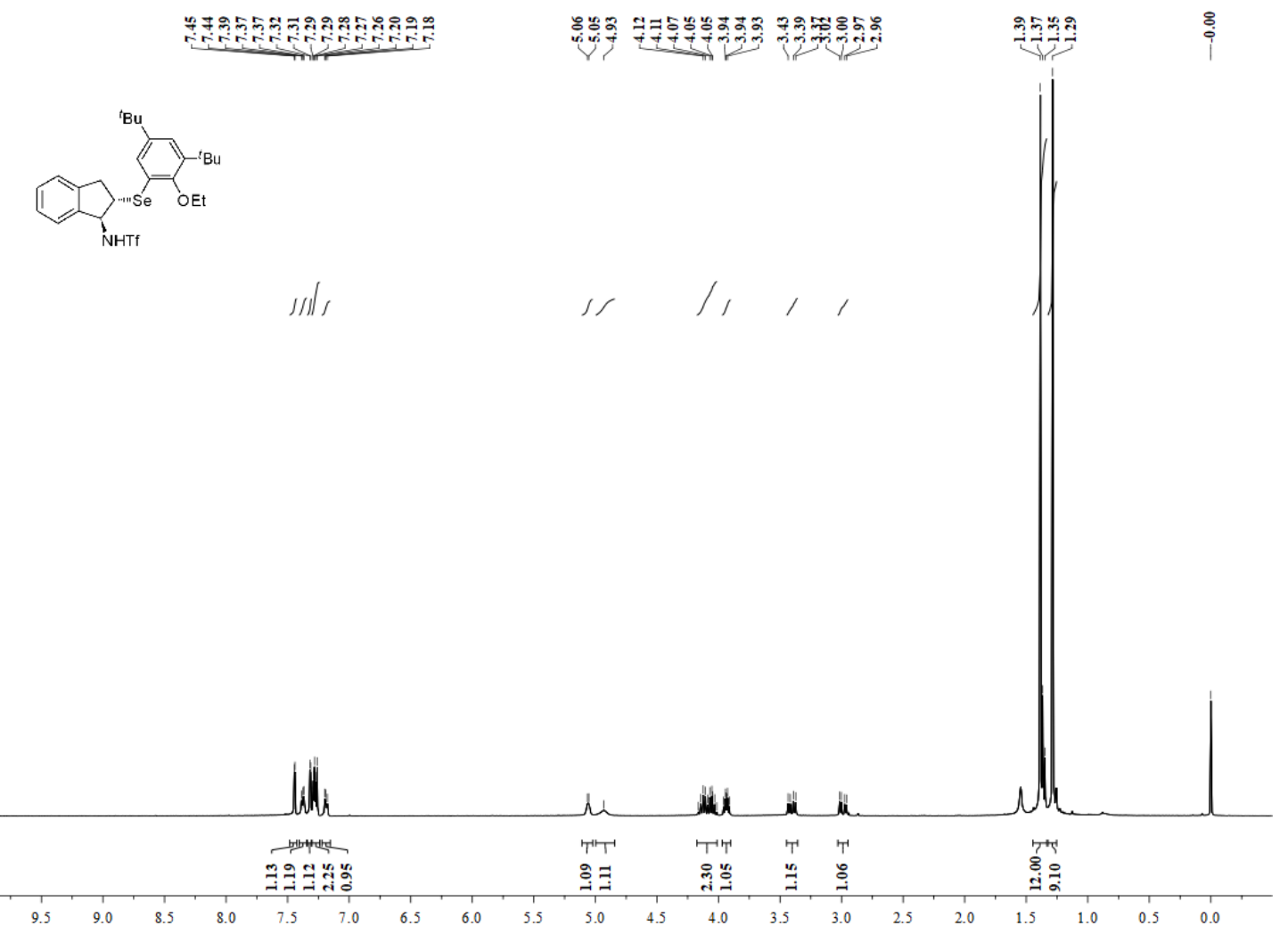

${ }^{13} \mathrm{C}$ NMR $\left(101 \mathrm{MHz}, \mathrm{CDCl}_{3}\right)$ spectrum of compound $\mathbf{C 1 4}$
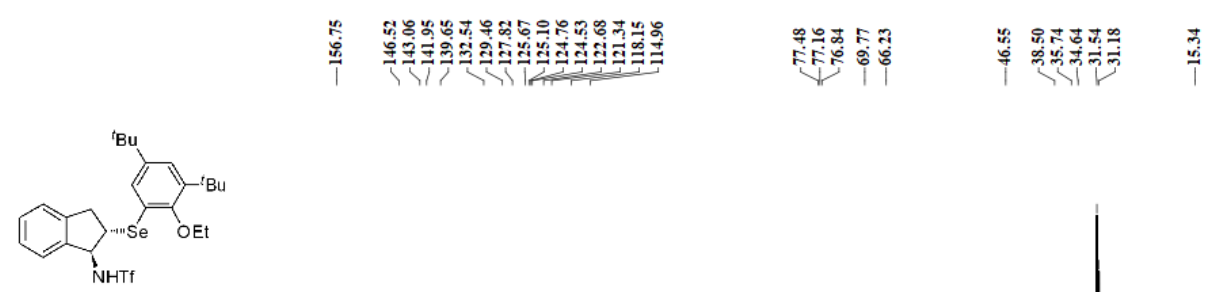

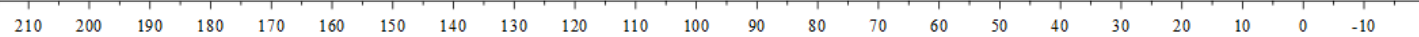


${ }^{1} \mathrm{H}$ NMR (400 MHz, $\mathrm{CDCl}_{3}$ ) spectrum of compound 3c

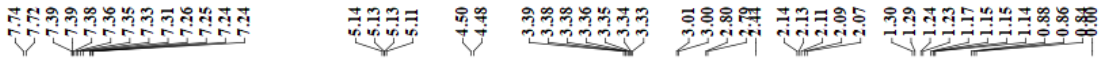

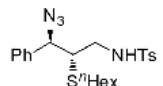

| $\mid$
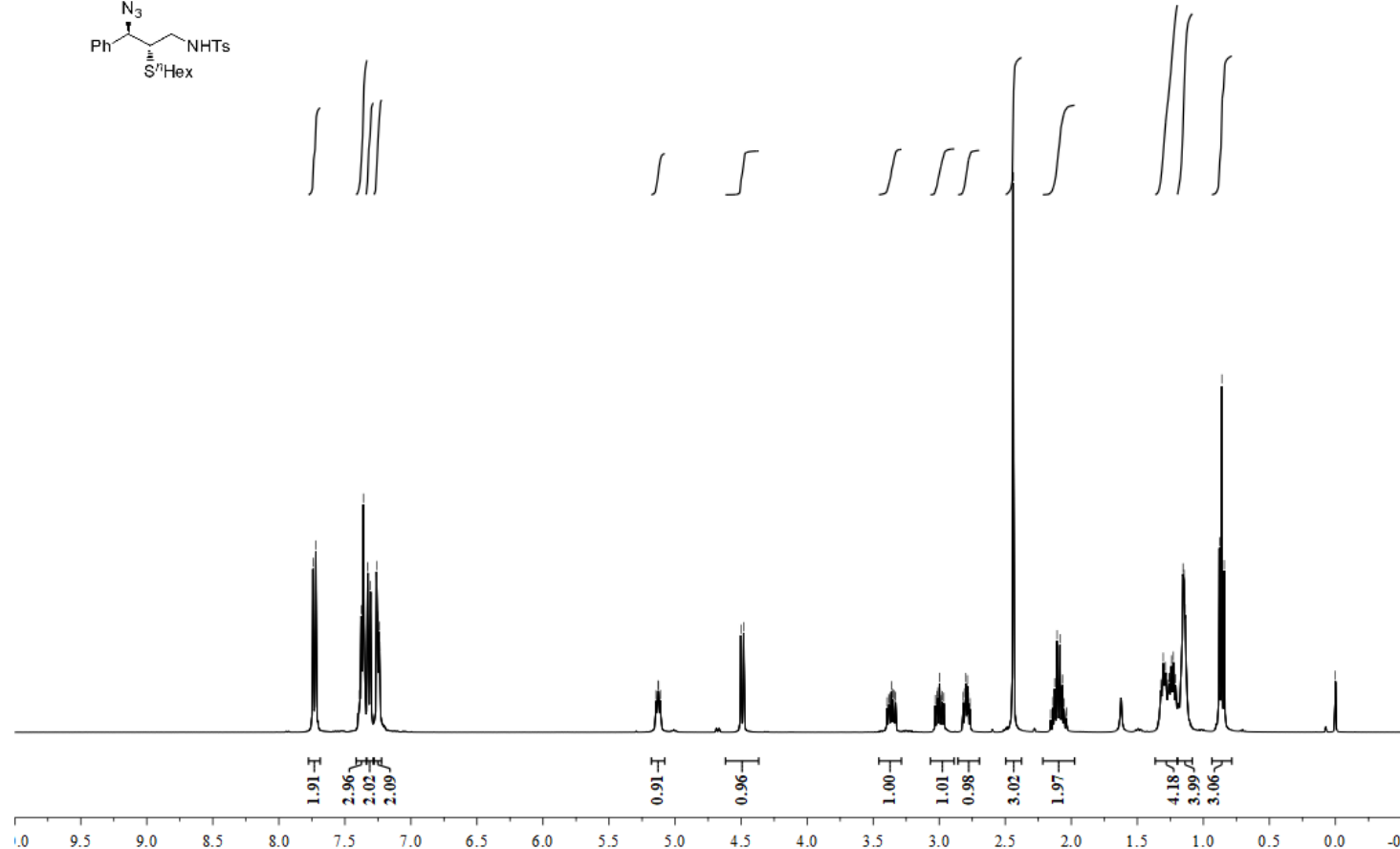

${ }^{13} \mathrm{C}$ NMR (101 MHz, $\mathrm{CDCl}_{3}$ ) spectrum of compound 3c

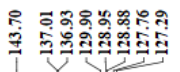

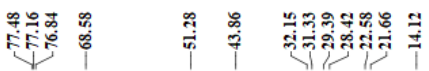

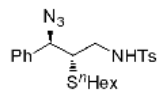

$\begin{array}{lllllllllllllllllllllll}210 & 200 & 190 & 180 & 170 & 160 & 150 & 140 & 130 & 120 & 110 & 100 & 90 & 80 & 70 & 60 & 50 & 40 & 30 & 20 & 10 & 0 & -10\end{array}$ 
${ }^{1} \mathrm{H}$ NMR (400 MHz, $\left.\mathrm{CDCl}_{3}\right)$ spectrum of compound $\mathbf{3 e}$

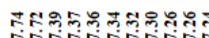

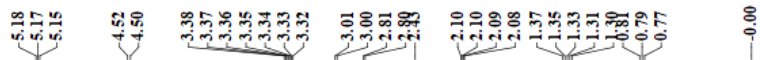

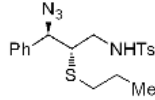
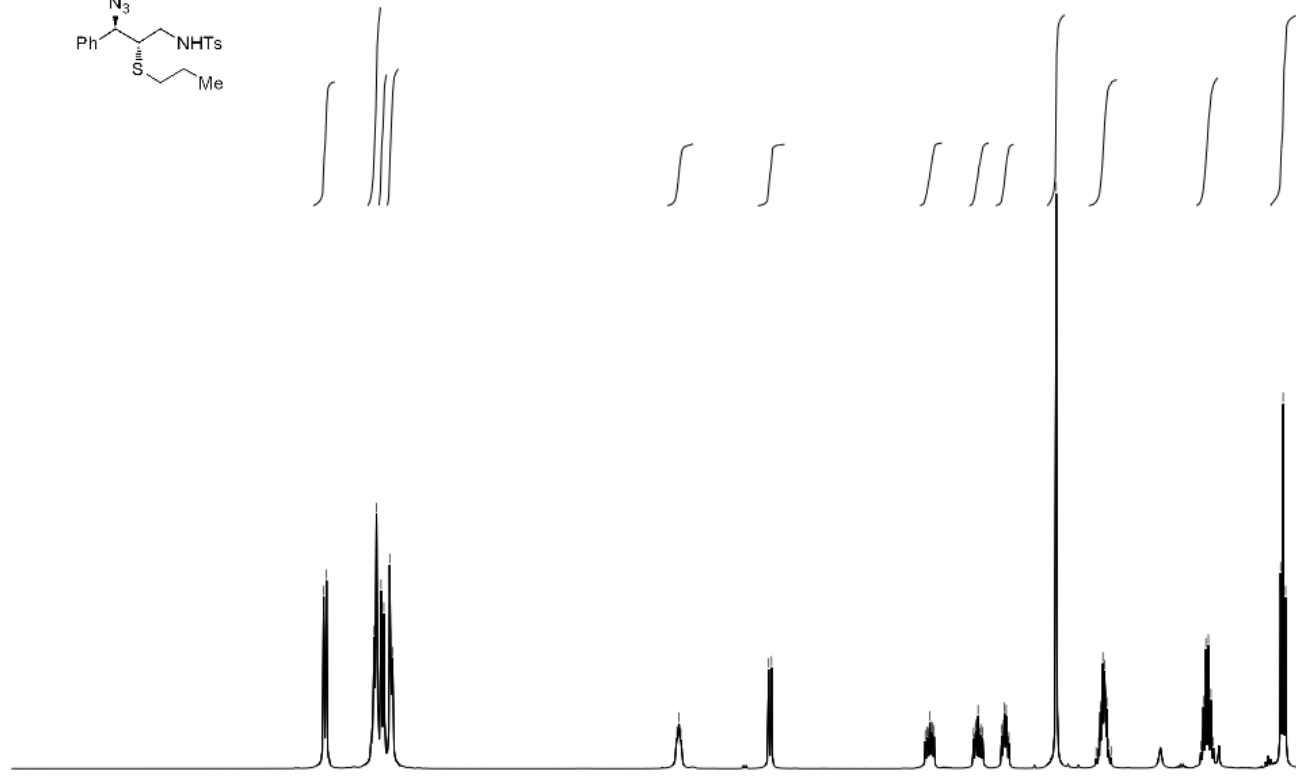

'T T⿳亠口冋.

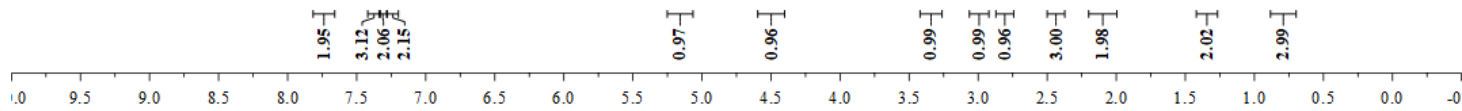

${ }^{13} \mathrm{C}$ NMR (101 MHz, $\left.\mathrm{CDCl}_{3}\right)$ spectrum of compound $\mathbf{3 e}$

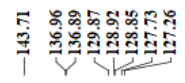

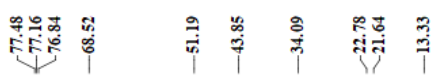

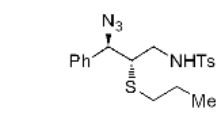

$\begin{array}{lllllllllllll}210 & 200 & 190 & 180 & 170 & 160 & 150 & 140 & 130 & 120 & 110 & 100 & 90\end{array}$

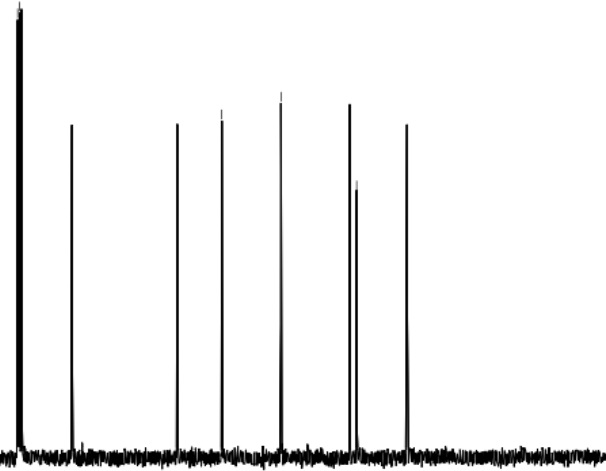


${ }^{1} \mathrm{H}$ NMR (400 MHz, $\mathrm{CDCl}_{3}$ ) spectrum of compound $\mathbf{3 f}$

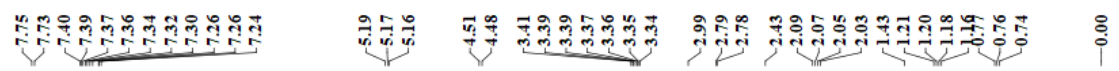

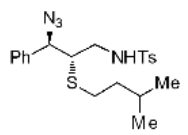<smiles>CC1C#CCC1</smiles>

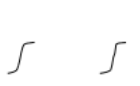

$\iiint \int$
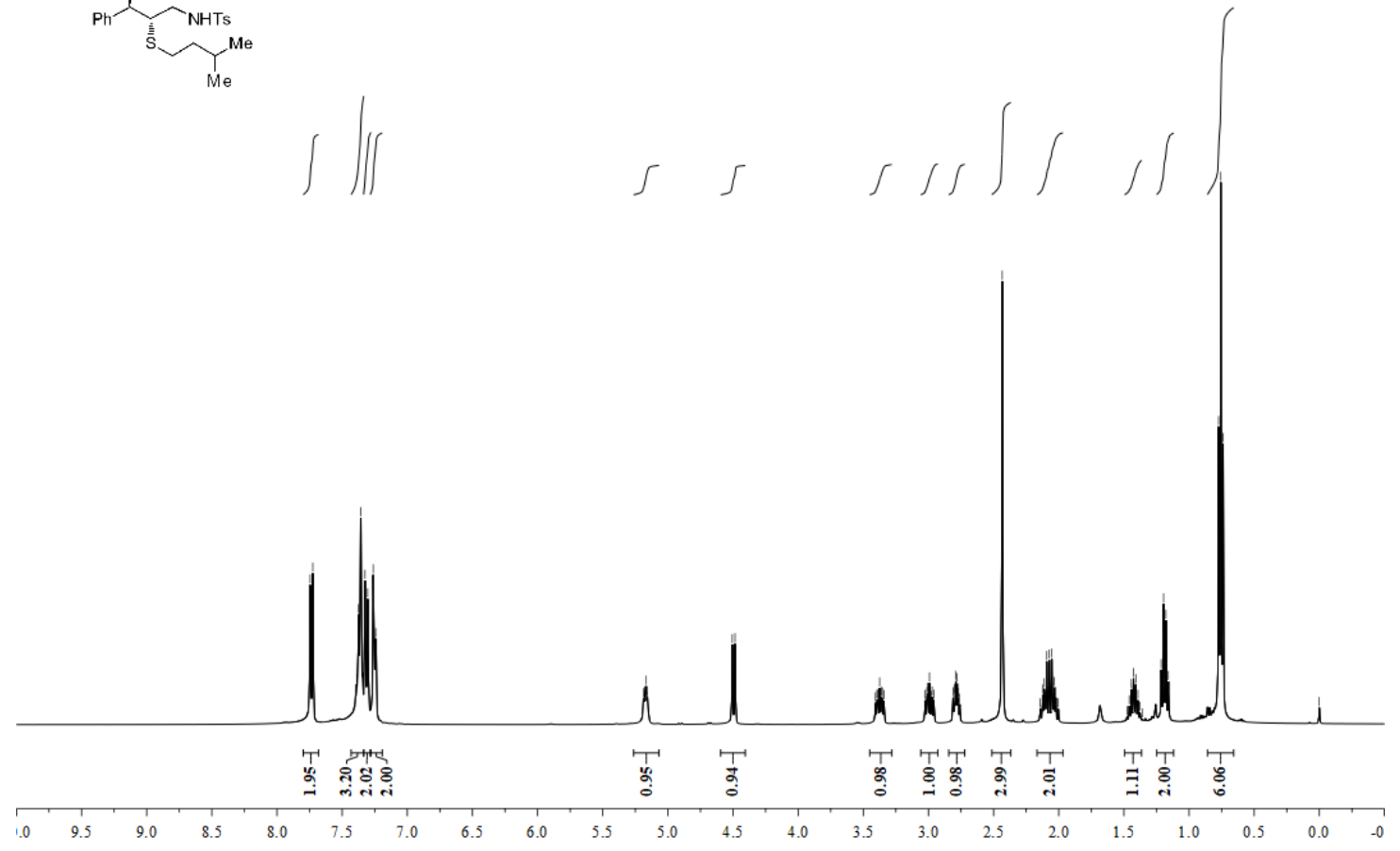

${ }^{13} \mathrm{C}$ NMR $\left(101 \mathrm{MHz}, \mathrm{CDCl}_{3}\right)$ spectrum of compound $\mathbf{3 f}$

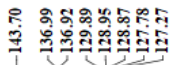

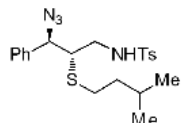

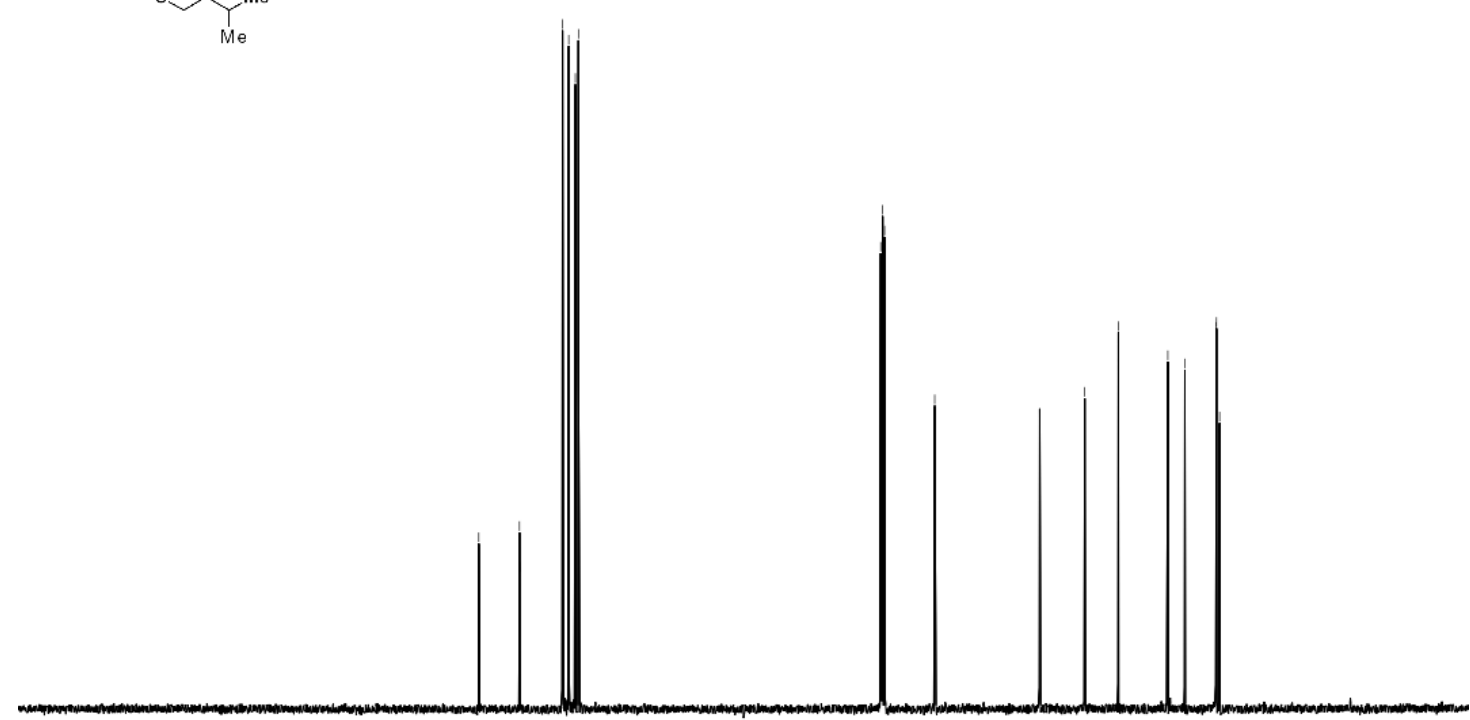

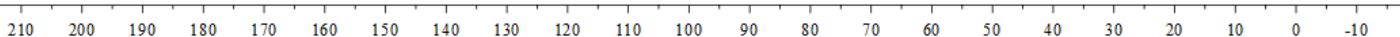


${ }^{1} \mathrm{H}$ NMR (400 MHz, $\mathrm{CDCl}_{3}$ ) spectrum of compound 3g

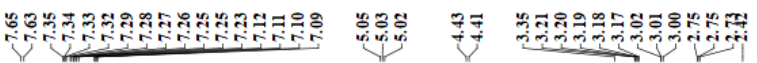

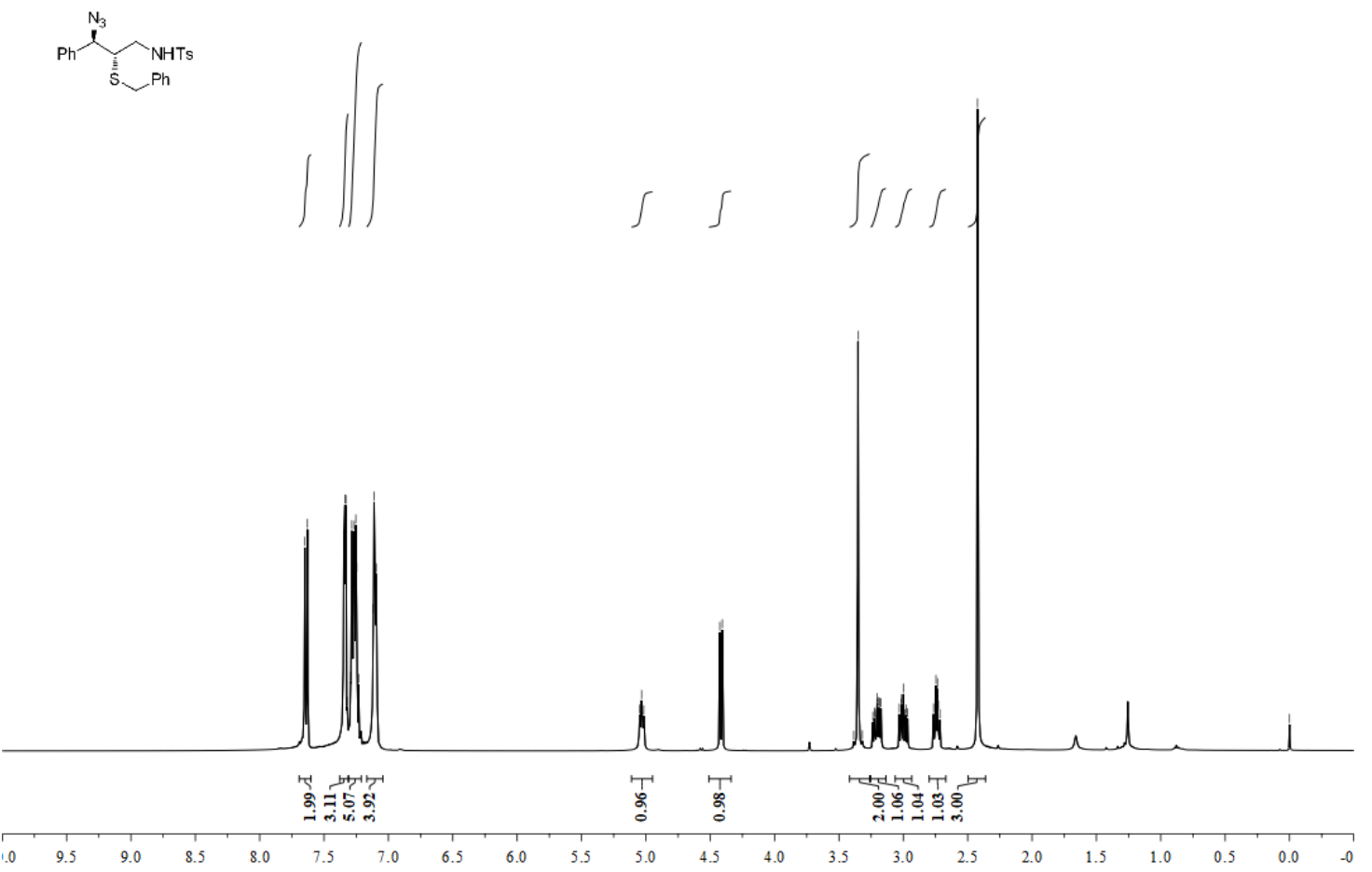

${ }^{13} \mathrm{C}$ NMR (101 MHz, $\left.\mathrm{CDCl}_{3}\right)$ spectrum of compound $\mathbf{3 g}$

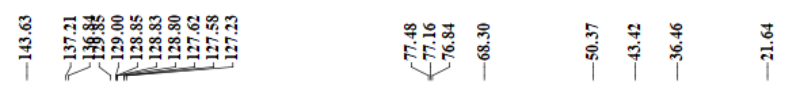<smiles>CSCC(C[NH2+])C(N)c1ccccc1</smiles>

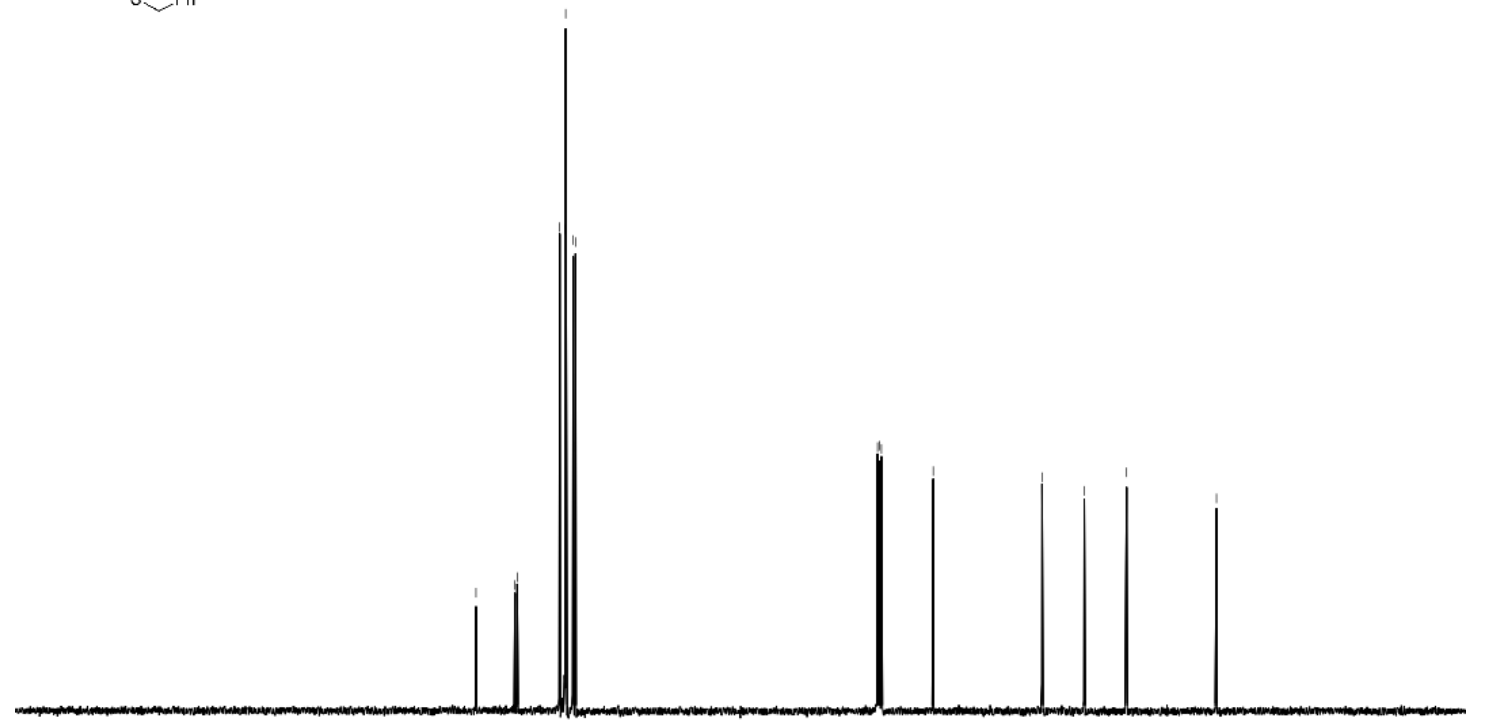

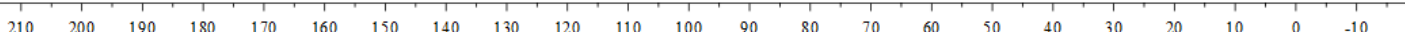


${ }^{1} \mathrm{H}$ NMR (400 MHz, $\mathrm{CDCl}_{3}$ ) spectrum of compound $\mathbf{3 h}$

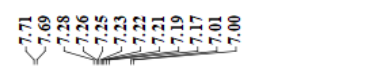

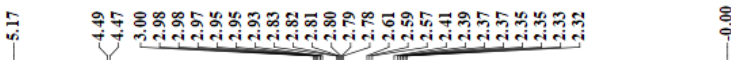

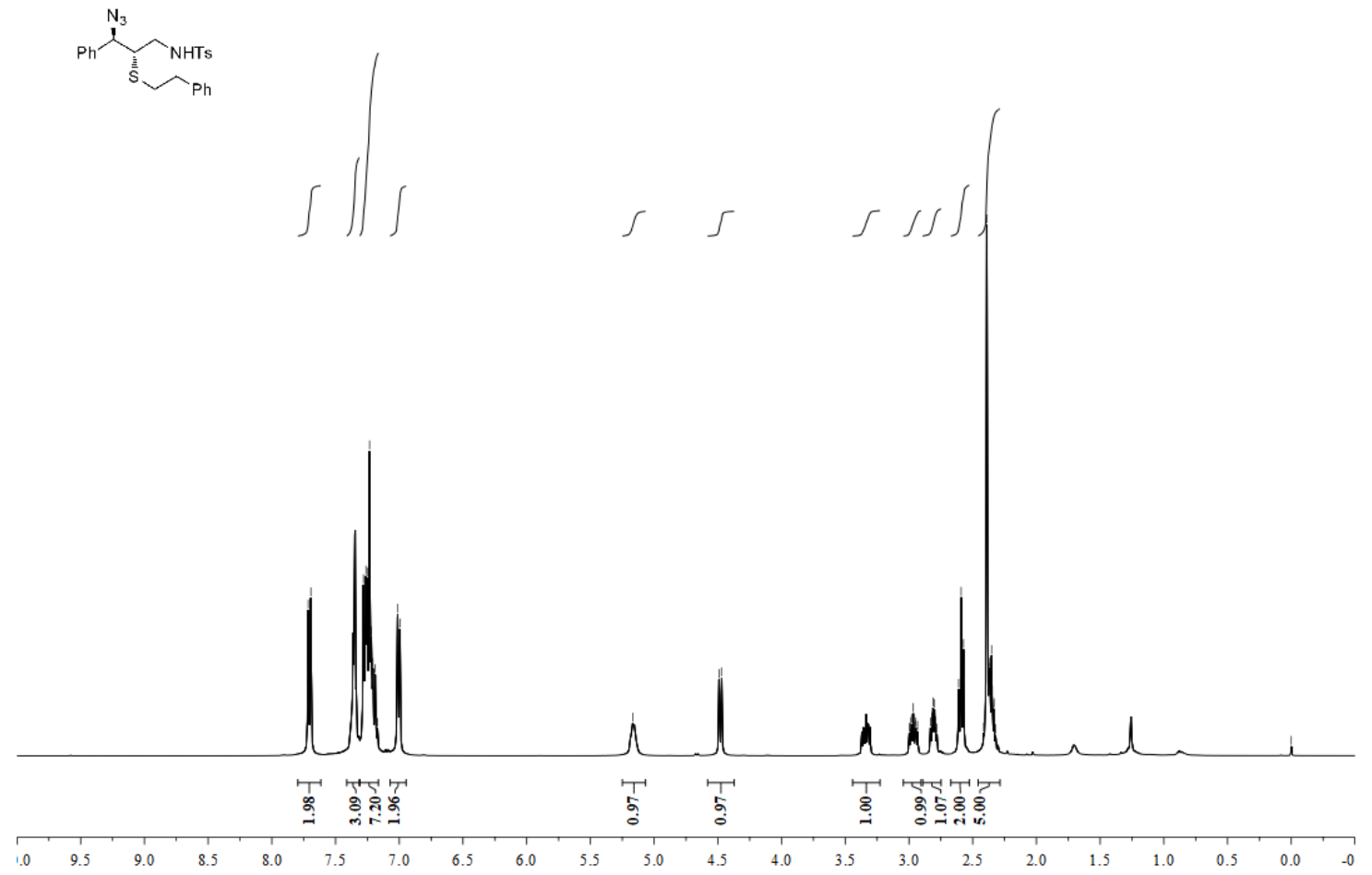

${ }^{13} \mathrm{C}$ NMR (101 MHz, $\left.\mathrm{CDCl}_{3}\right)$ spectrum of compound $\mathbf{3 h}$

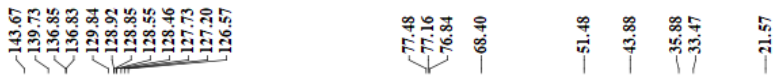
$\overbrace{\text { Sh }}^{N_{\mathrm{NHT}}^{\mathrm{N}_{3}}}$

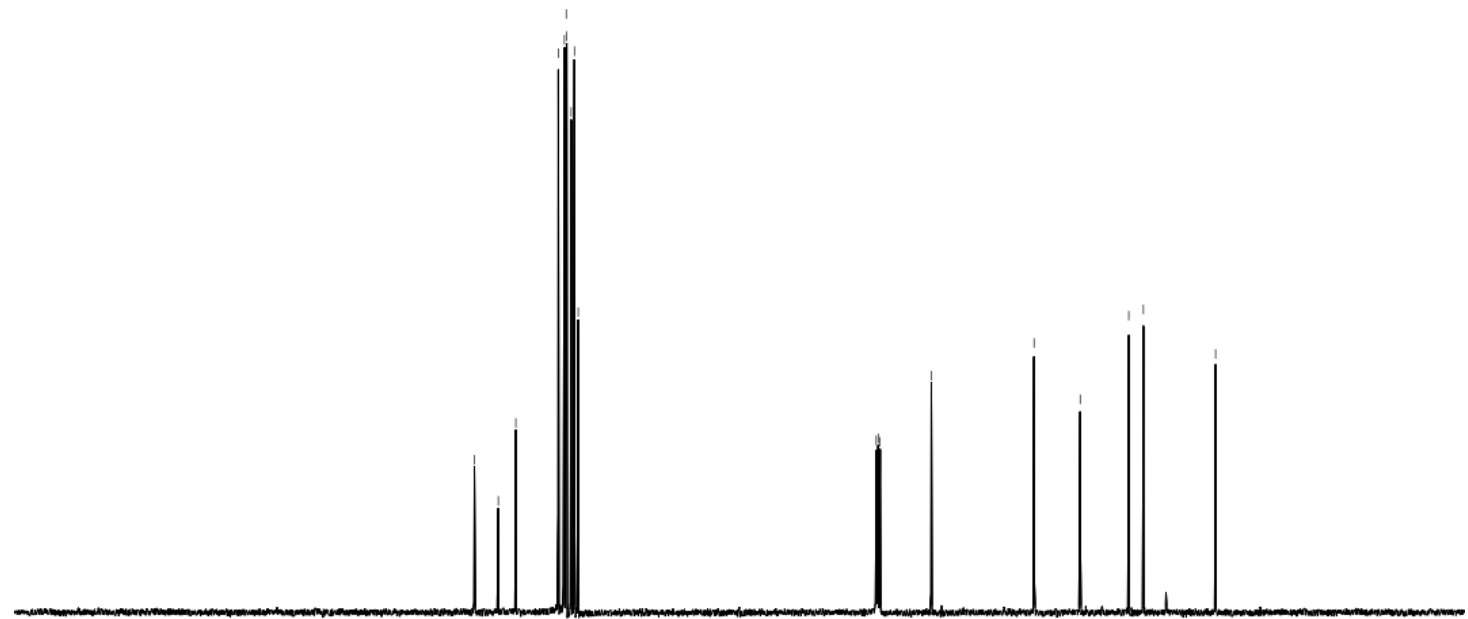

$\begin{array}{llllllllllllllllllllllll}210 & 200 & 190 & 180 & 170 & 160 & 150 & 140 & 130 & 120 & 110 & 100 & 90 & 30 & 70 & 60 & 50 & 40 & 30 & 20 & 10 & 0 & -10\end{array}$ 
${ }^{1} \mathrm{H}$ NMR (400 MHz, $\mathrm{CDCl}_{3}$ ) spectrum of compound 3i

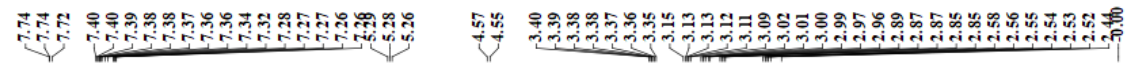
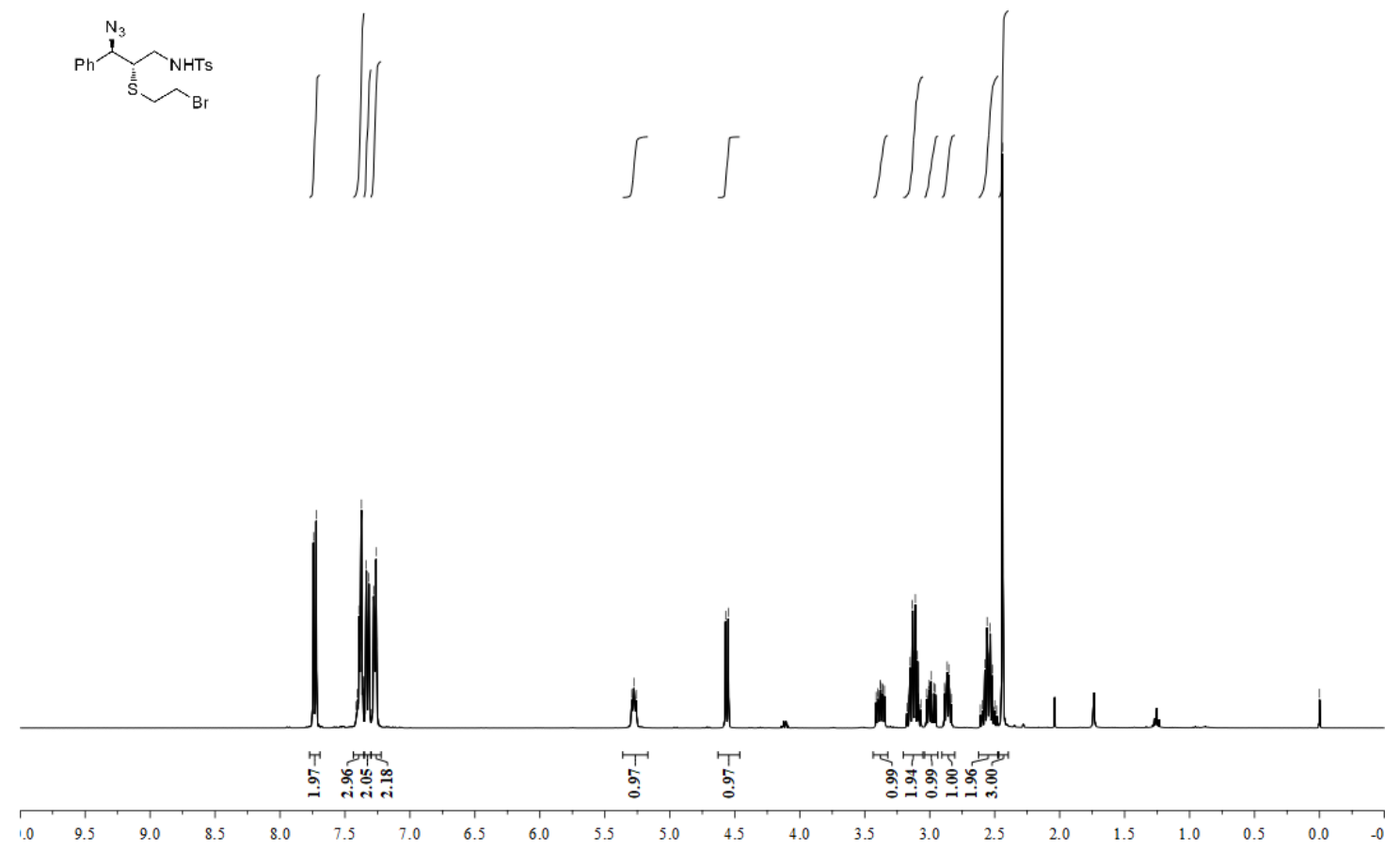

${ }^{13} \mathrm{C}$ NMR $\left(101 \mathrm{MHz}, \mathrm{CDCl}_{3}\right)$ spectrum of compound 3i

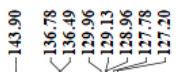

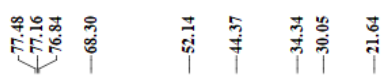

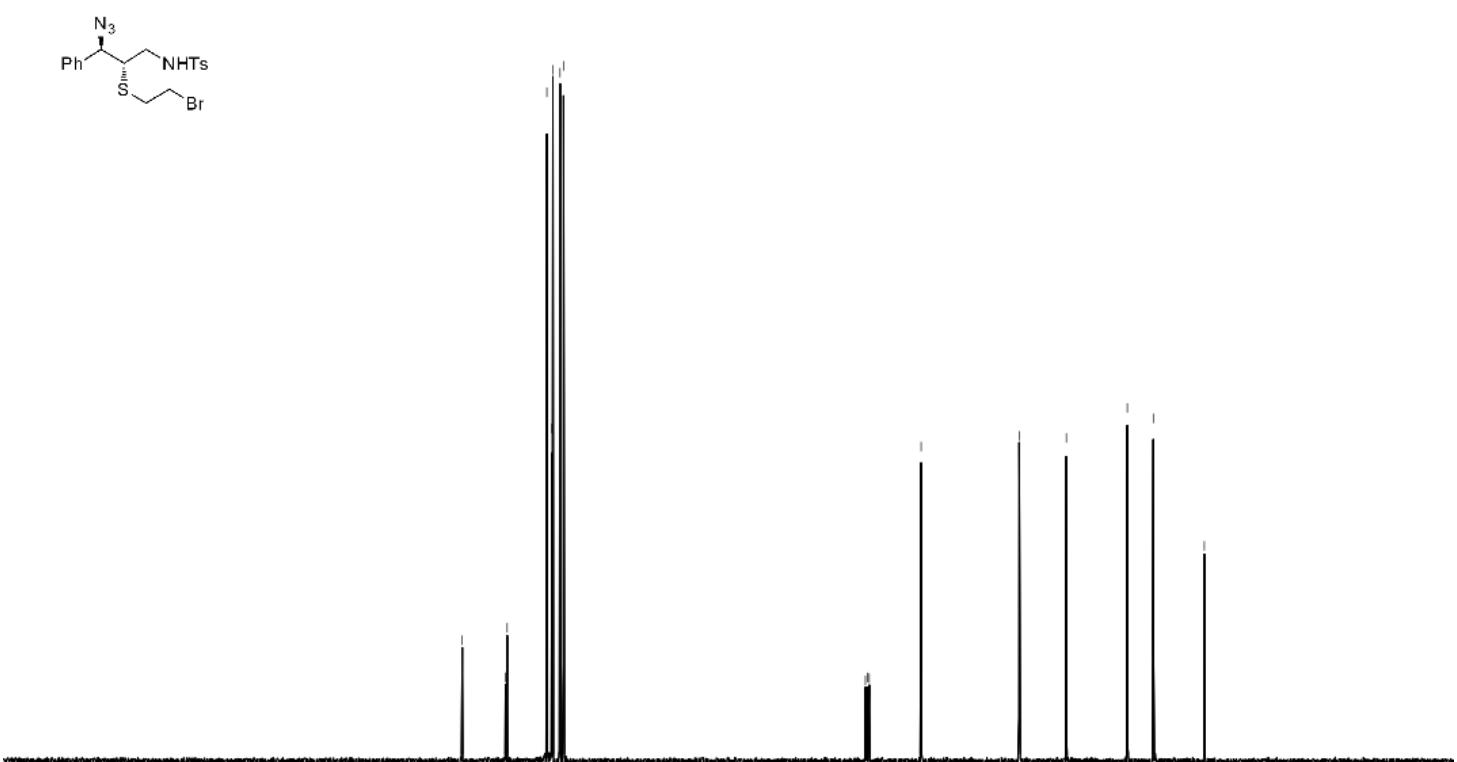

$\begin{array}{lllllllllllllllllllllll}210 & 200 & 190 & 180 & 170 & 160 & 150 & 140 & 130 & 120 & 110 & 100 & 90 & 80 & 70 & 60 & 50 & 40 & 30 & 20 & 10 & 0\end{array}$ 
${ }^{1} \mathrm{H}$ NMR (400 MHz, $\mathrm{CDCl}_{3}$ ) spectrum of compound $\mathbf{3 j}$

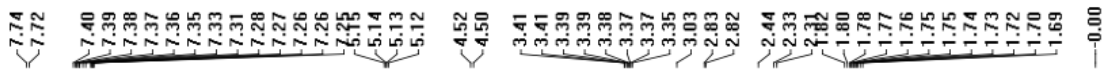

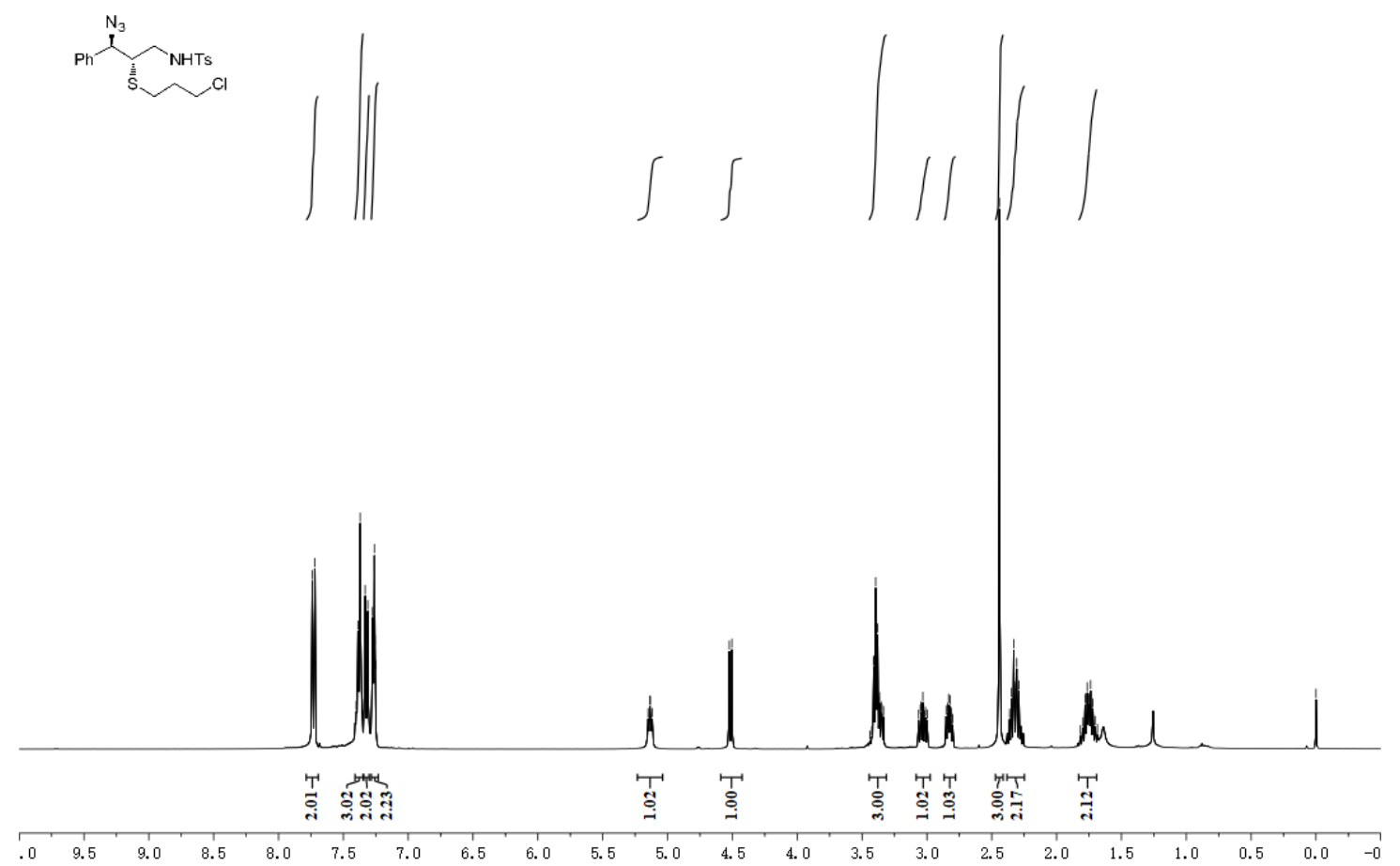

${ }^{13} \mathrm{C}$ NMR (101 MHz, $\mathrm{CDCl}_{3}$ ) spectrum of compound $\mathbf{3 j}$

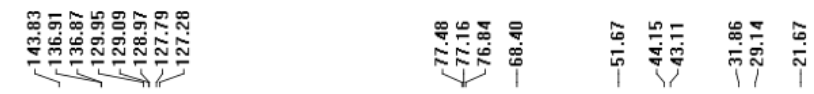

$\overbrace{\text { Sh }}^{\mathrm{N}_{\mathrm{NHTS}}}$

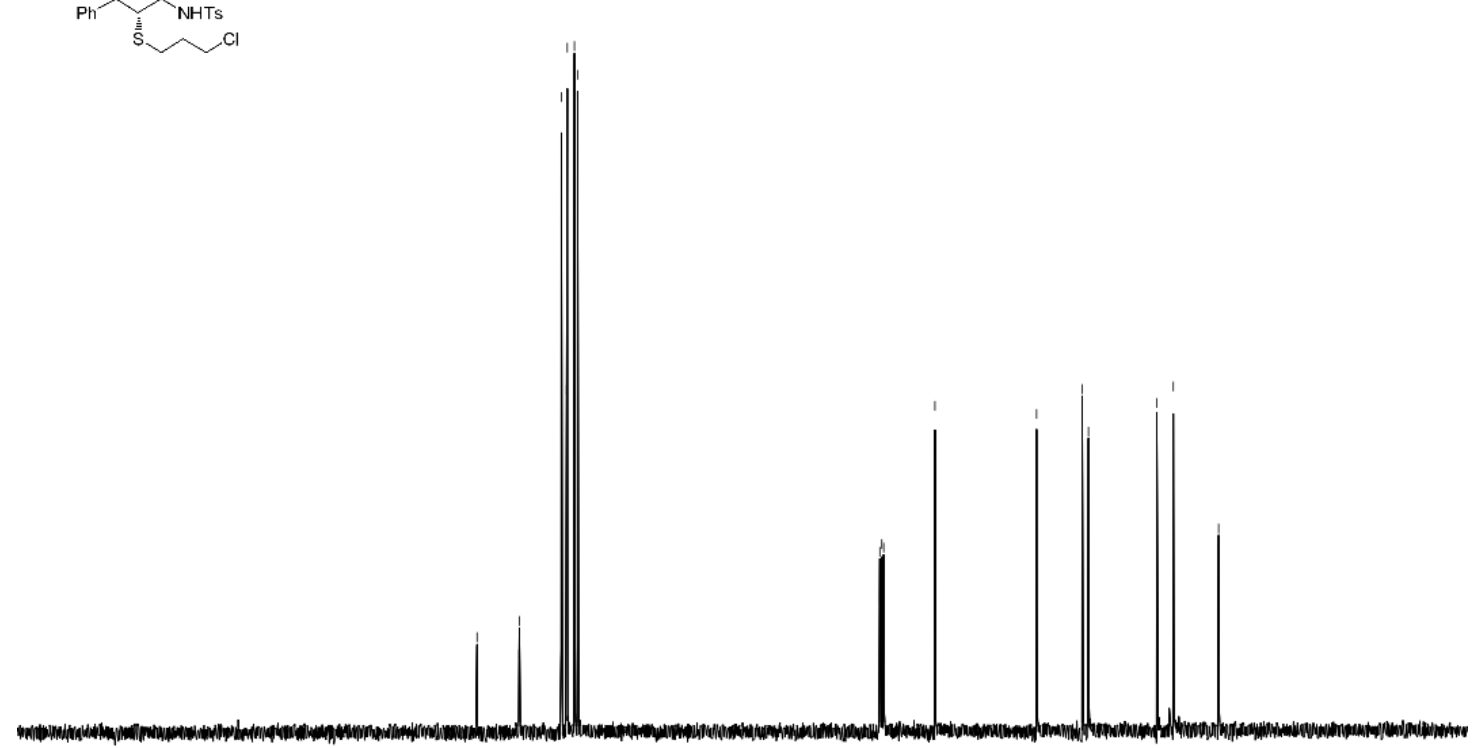

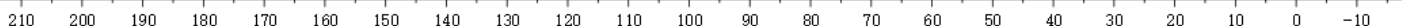


${ }^{1} \mathrm{H}$ NMR (400 MHz, $\mathrm{CDCl}_{3}$ ) spectrum of compound 3k

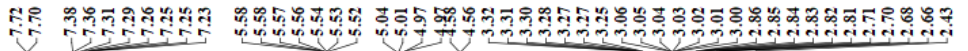<smiles>CCCCC(C)CCC</smiles>
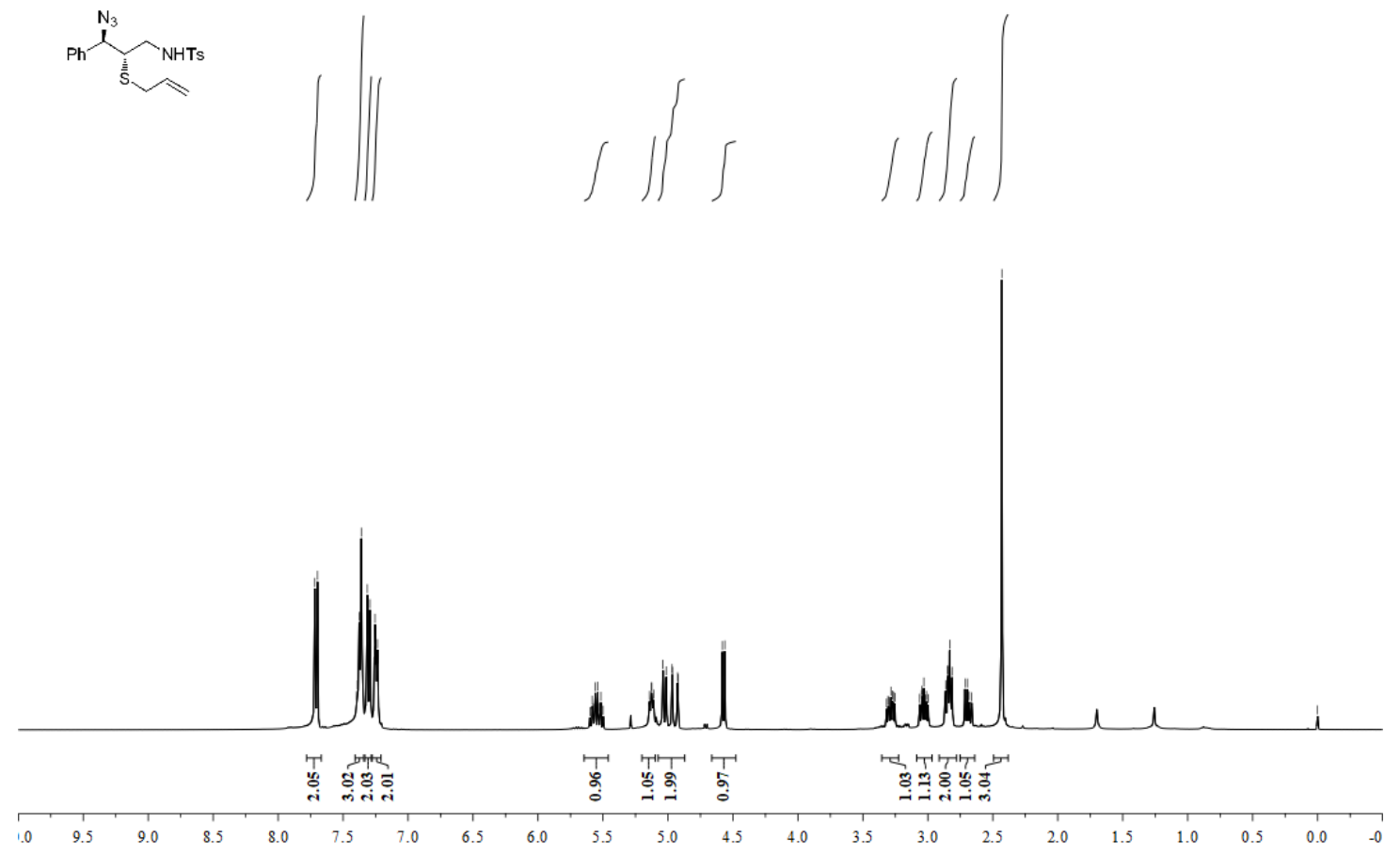

${ }^{13} \mathrm{C}$ NMR (101 MHz, $\left.\mathrm{CDCl}_{3}\right)$ spectrum of compound 3k

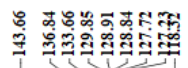

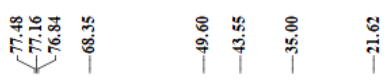

$\overbrace{\text { ShH }}^{\mathrm{N}_{3}}$

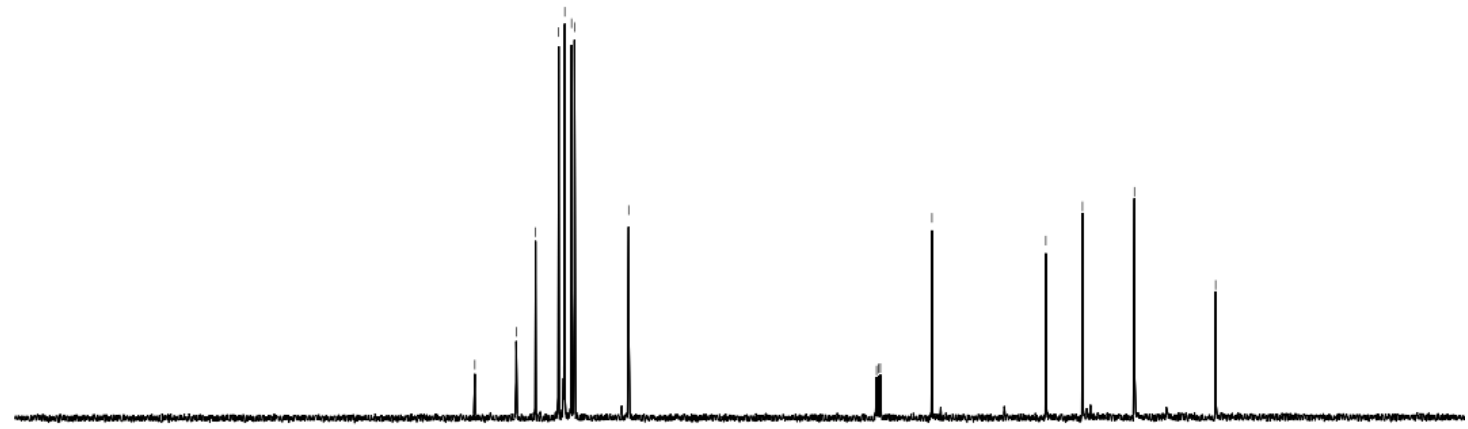

$\begin{array}{lllllllllllllllllllllll}210 & 200 & 190 & 180 & 170 & 160 & 150 & 140 & 130 & 120 & 110 & 100 & 90 & 80 & 70 & 60 & 50 & 40 & 30 & 20 & 10 & 0 & -10\end{array}$ 
${ }^{1} \mathrm{H} \mathrm{NMR}\left(400 \mathrm{MHz}, \mathrm{CDCl}_{3}\right.$ ) spectrum of compound $\mathbf{3 l}$

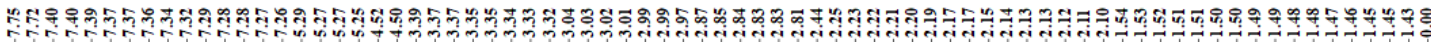

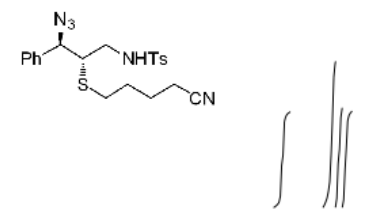
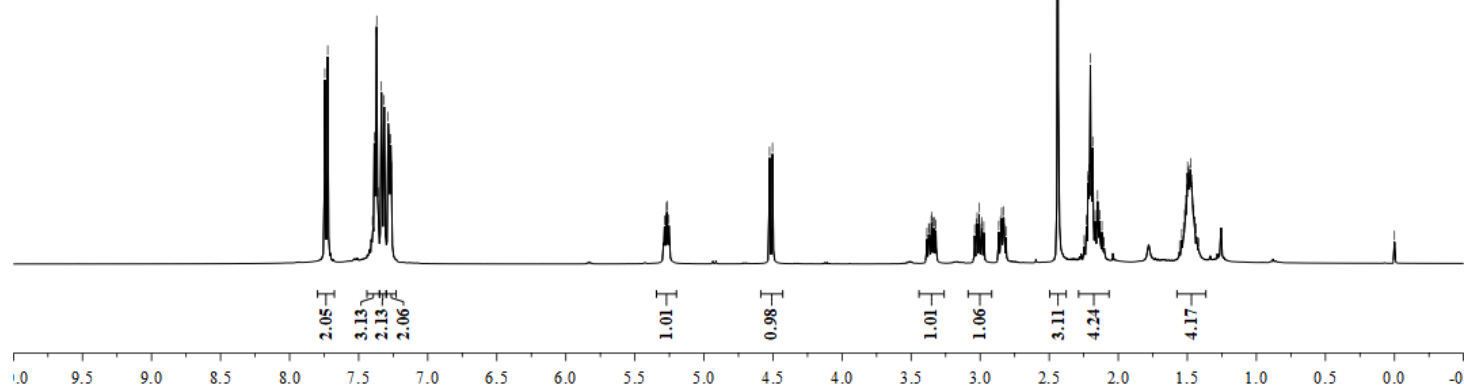

${ }^{13} \mathrm{C}$ NMR (101 MHz, $\left.\mathrm{CDCl}_{3}\right)$ spectrum of compound 31

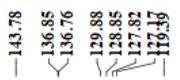

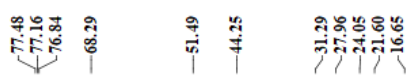

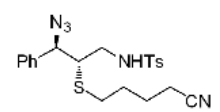


${ }^{1} \mathrm{H}$ NMR (400 MHz, $\mathrm{CDCl}_{3}$ ) spectrum of compound 3m

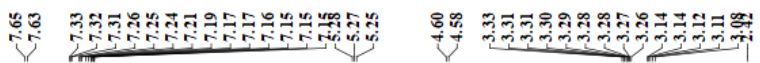
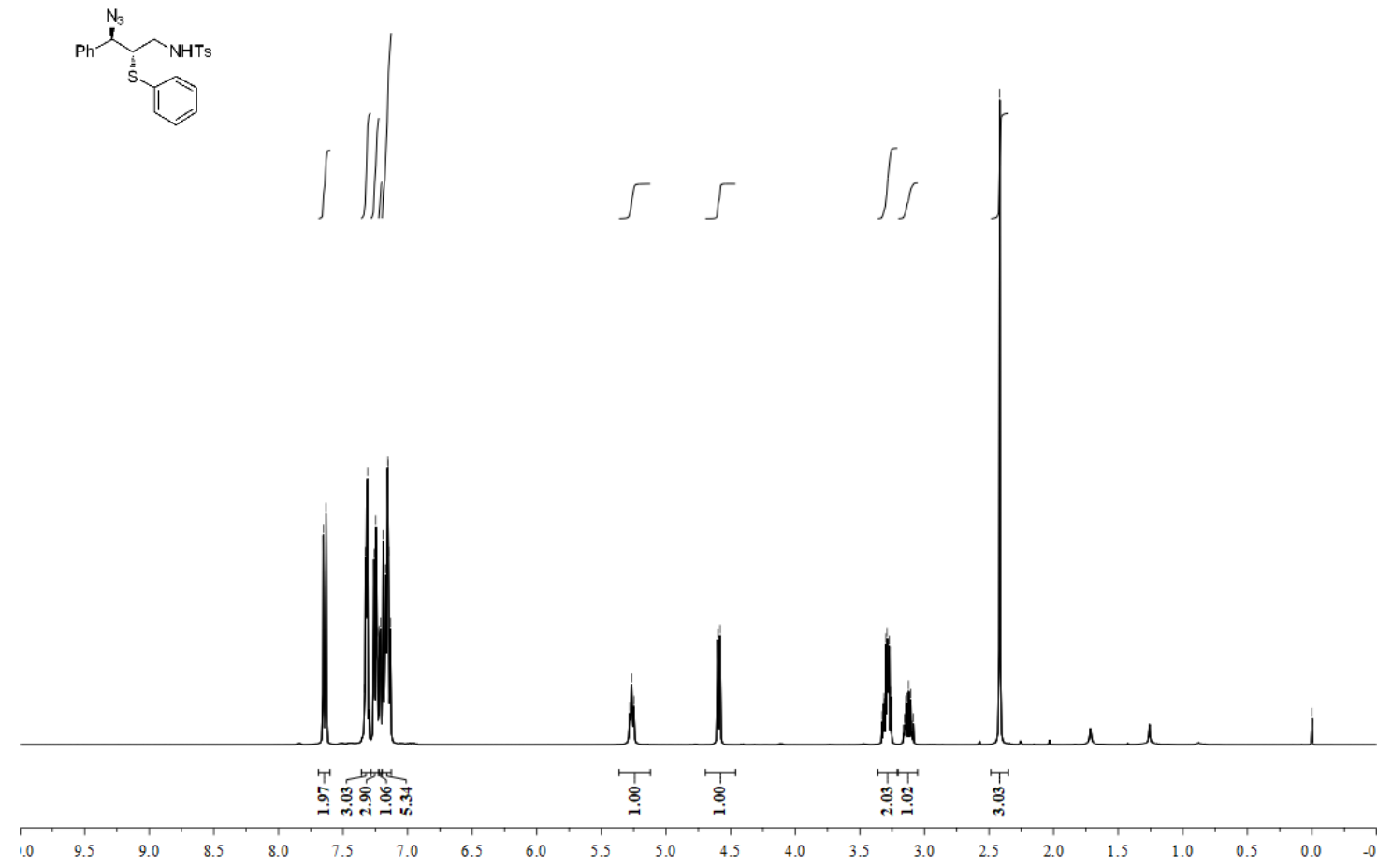

${ }^{13} \mathrm{C}$ NMR (101 MHz, $\mathrm{CDCl}_{3}$ ) spectrum of compound 3m

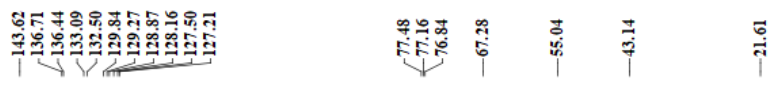<smiles>NCC(N)Cc1ccccc1</smiles>

$\begin{array}{lllllllllllllllllllllll}210 & 200 & 190 & 180 & 170 & 160 & 150 & 140 & 130 & 120 & 110 & 100 & 90 & 80 & 70 & 60 & 50 & 40 & 30 & 20 & 10 & 0 & -10\end{array}$ 
${ }^{1} \mathrm{H}$ NMR (400 MHz. $\mathrm{CDCl}_{2}$ ) snectrum of compound 3n
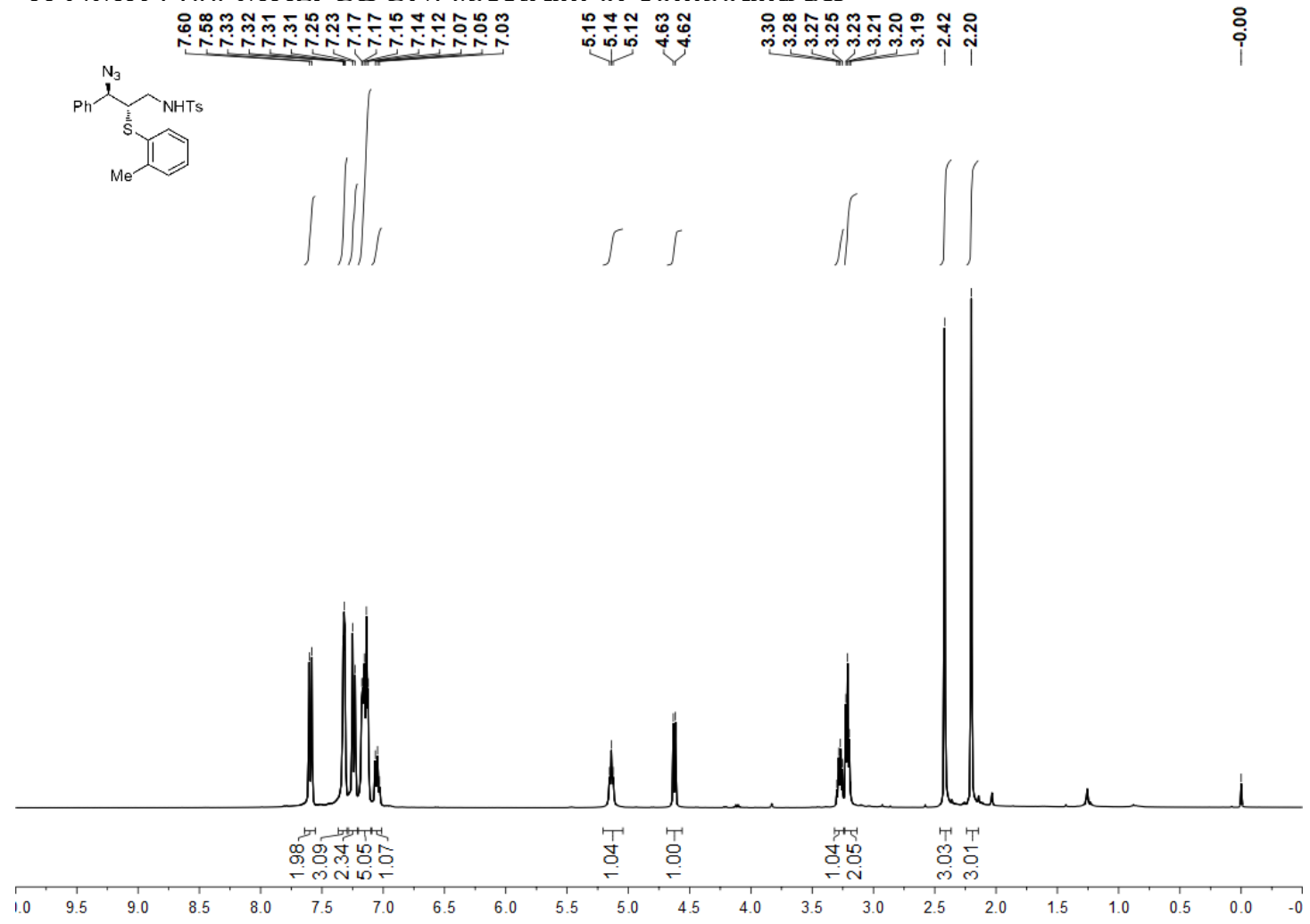

${ }^{13} \mathrm{C}$ NMR (101 MHz. CDCl $\mathrm{CD}_{2}$ ) spectrum of comnound 3n

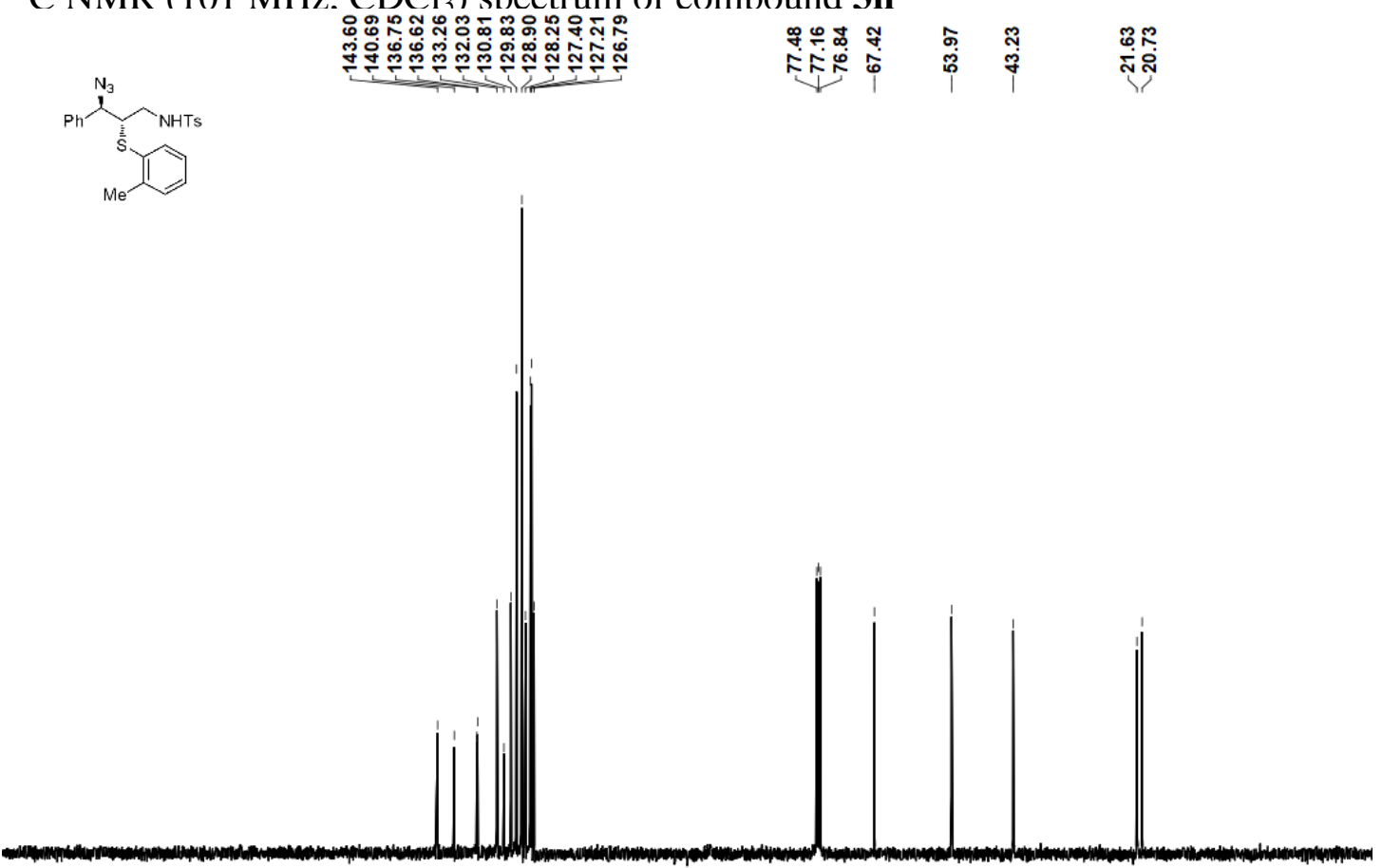

$\begin{array}{llllllllllllllllllllllllll}210 & 200 & 190 & 180 & 170 & 160 & 150 & 140 & 130 & 120 & 110 & 100 & 90 & 80 & 70 & 60 & 50 & 40 & 30 & 20 & 10 & 0 & -10\end{array}$ 
${ }^{1} \mathrm{H}$ NMR (400 MHz, $\mathrm{CDCl}_{3}$ ) spectrum of compound 3o

品

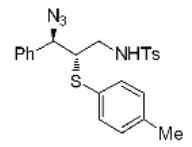<smiles>c1ccccc1</smiles>
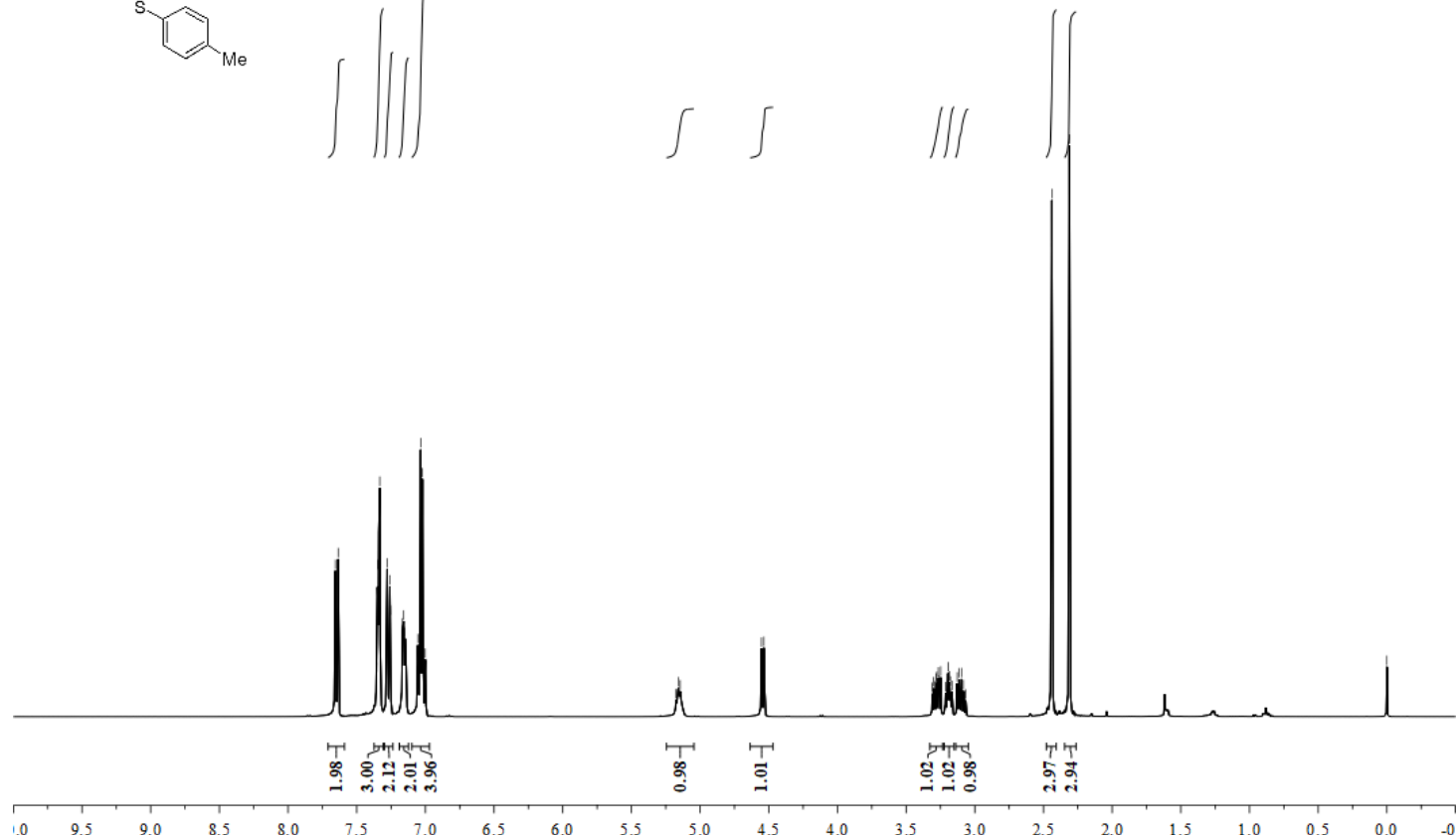

${ }^{13} \mathrm{C}$ NMR (101 MHz, $\mathrm{CDCl}_{3}$ ) spectrum of compound 3o

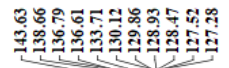

NHTs

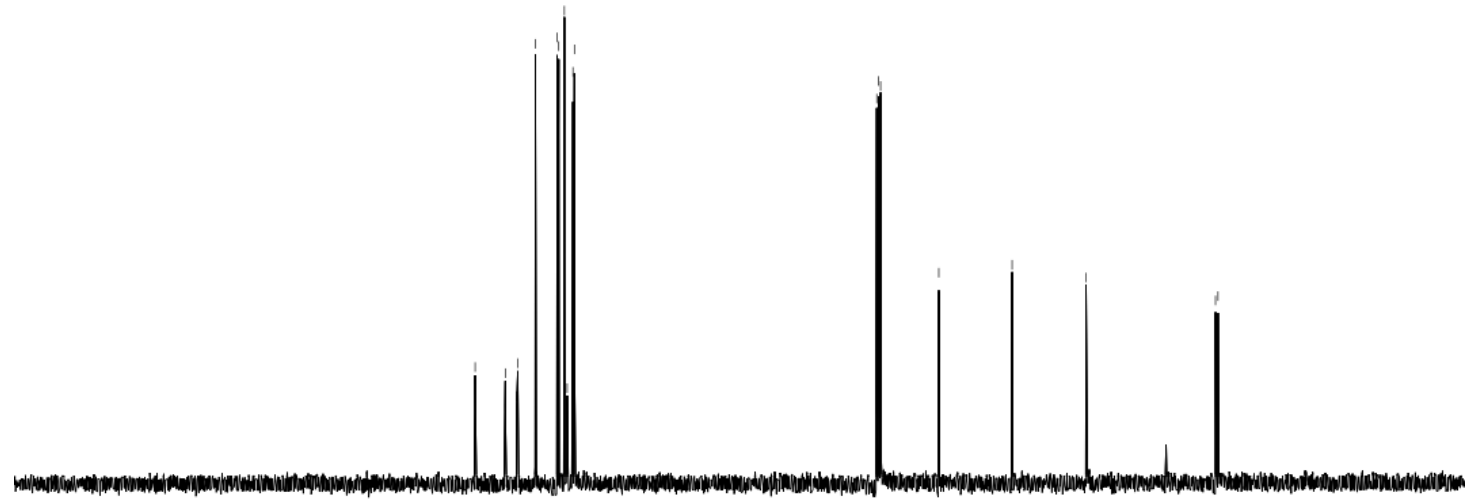

$\begin{array}{llllllllllllllllllllllll}210 & 200 & 190 & 180 & 170 & 160 & 150 & 140 & 130 & 120 & 110 & 100 & 90 & 80 & 70 & 60 & 50 & 40 & 30 & 20 & 10 & 0\end{array}$ 
${ }^{1} \mathrm{H}$ NMR (400 MHz, $\mathrm{CDCl}_{3}$ ) spectrum of compound $\mathbf{3 p}$

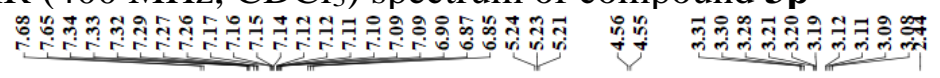

$\stackrel{8}{i}$
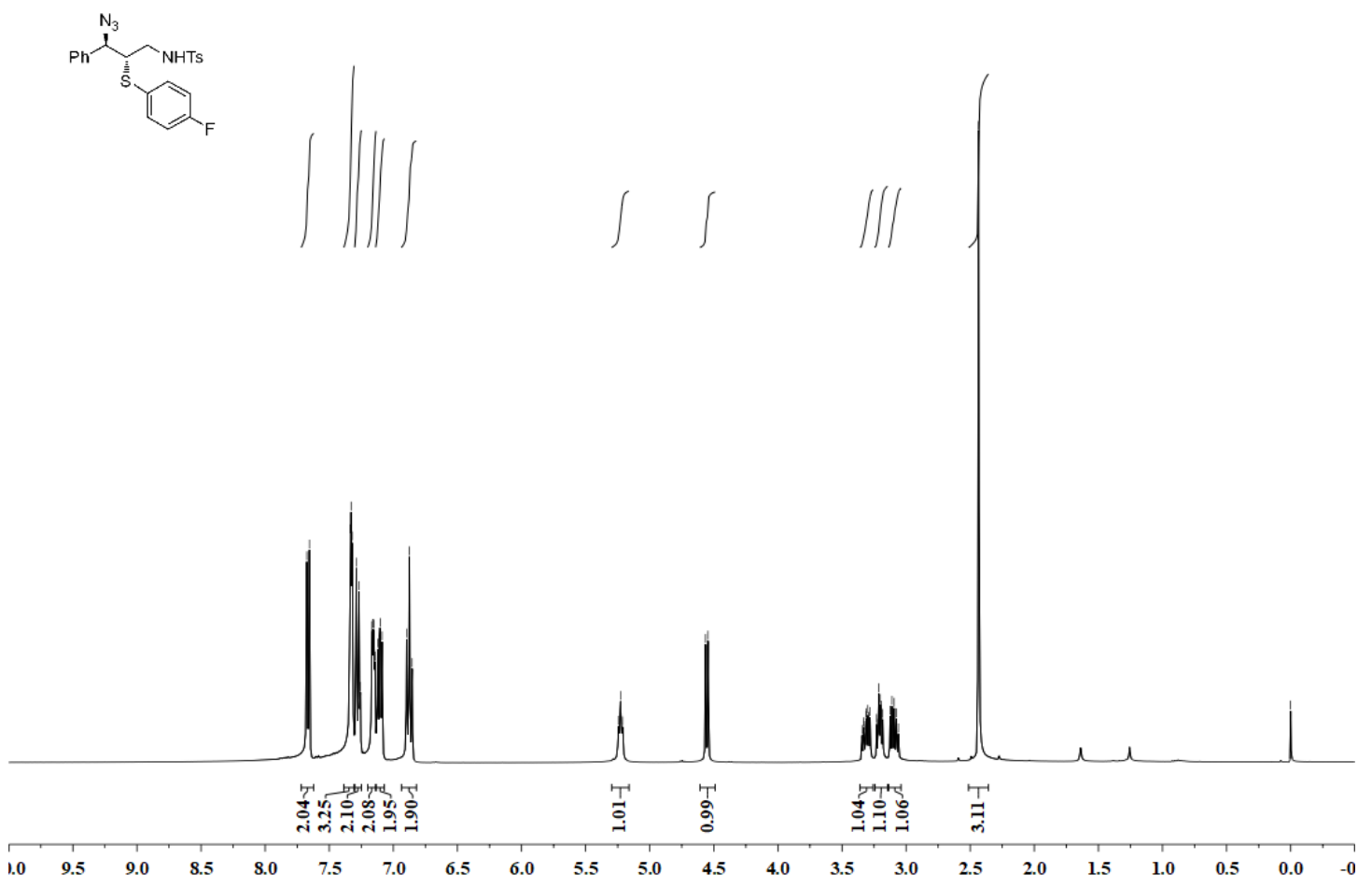

${ }^{13} \mathrm{C}$ NMR (101 MHz, $\mathrm{CDCl}_{3}$ ) spectrum of compound $\mathbf{3 p}$

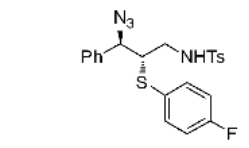

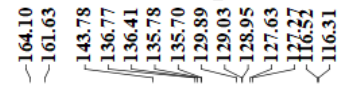

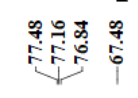

7.

索
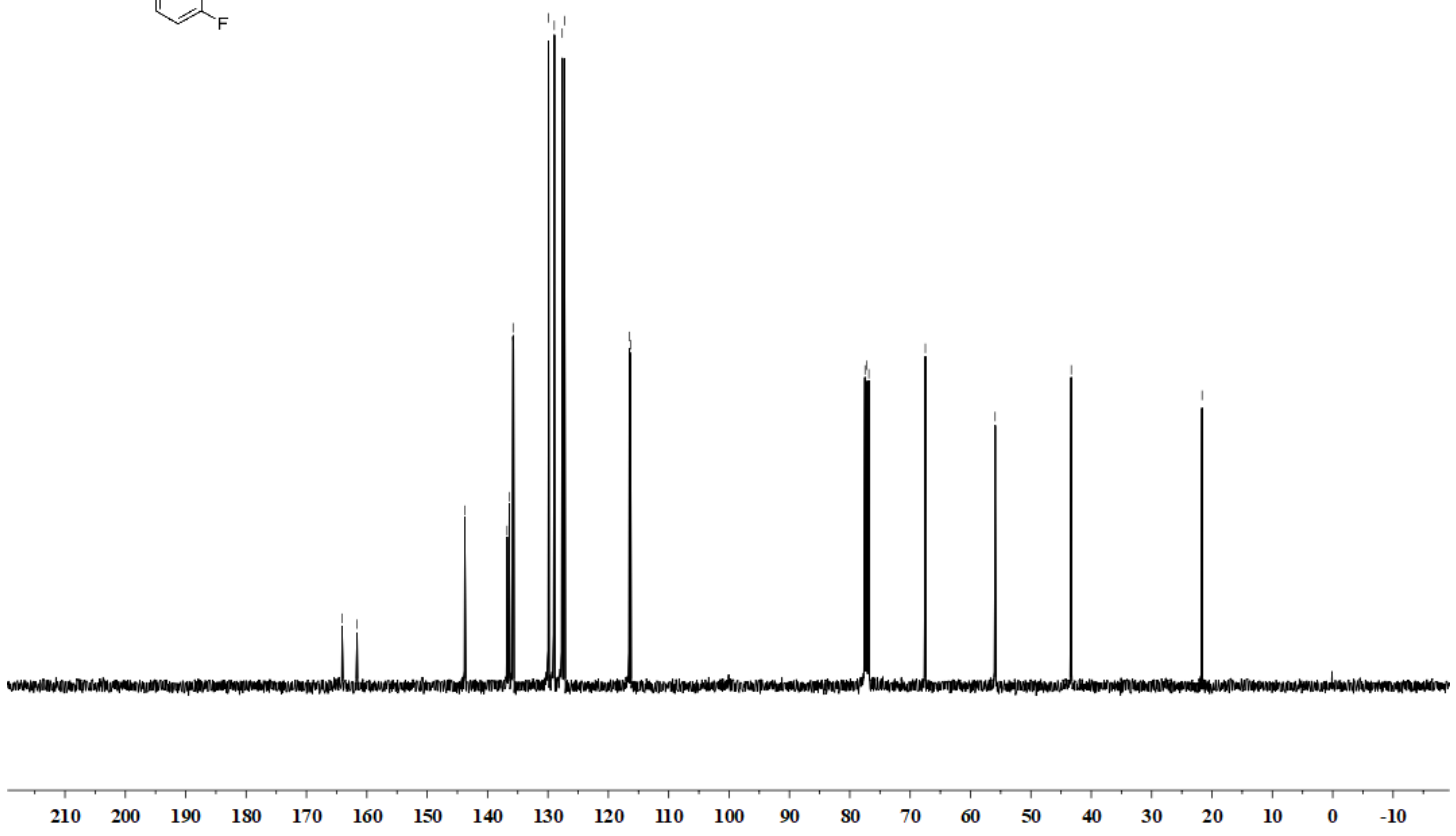

S96 
${ }^{1} \mathrm{H}$ NMR (400 MHz, $\mathrm{CDCl}_{3}$ ) spectrum of compound 3q

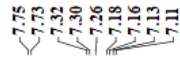

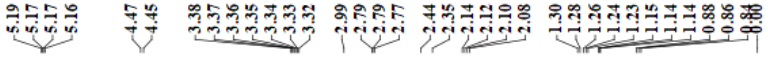

$\overbrace{M e}^{N_{3}} \overbrace{S_{H A X}}^{N_{H A S}}$

$\iint||$
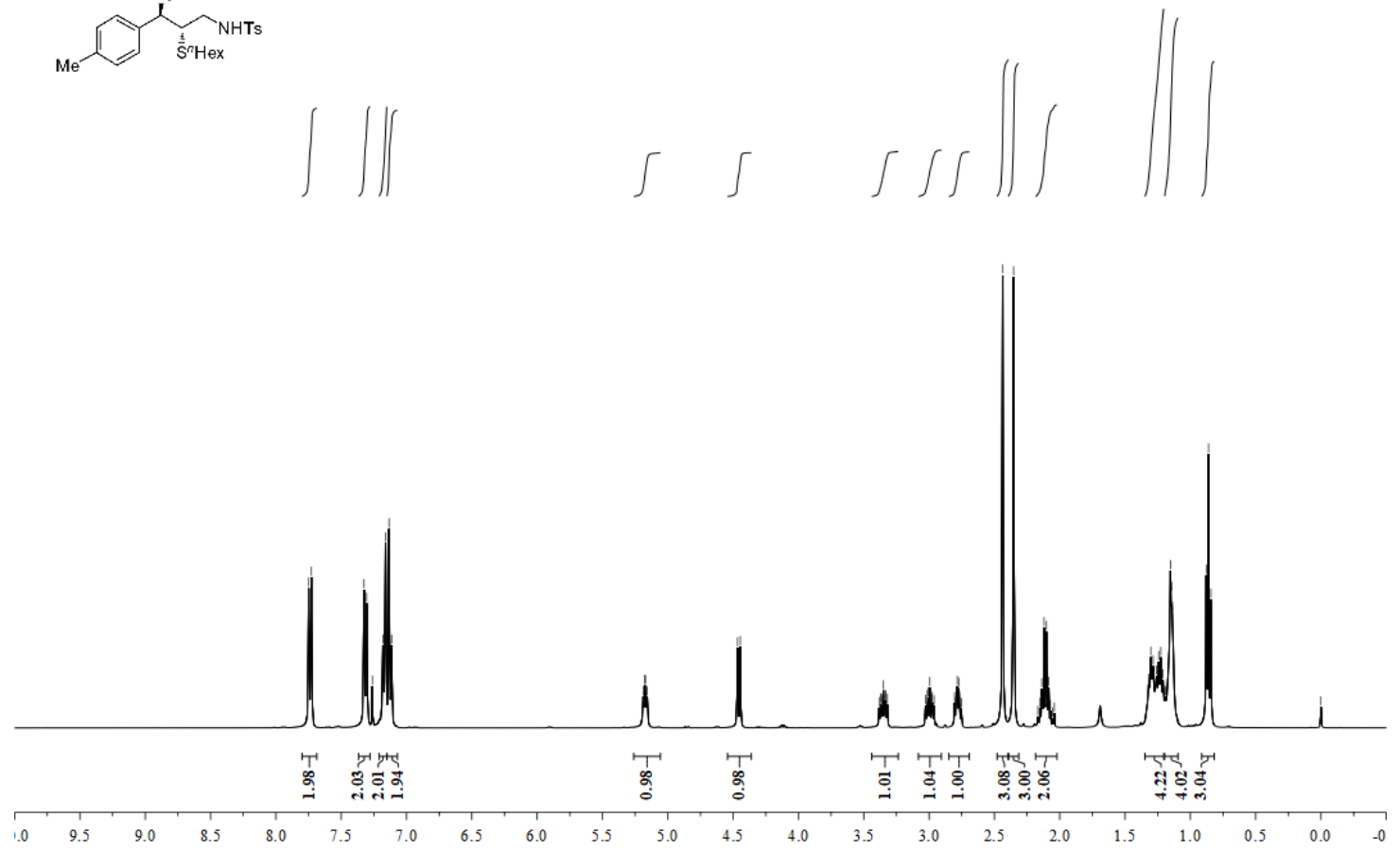

${ }^{13} \mathrm{C}$ NMR (101 MHz, $\left.\mathrm{CDCl}_{3}\right)$ spectrum of compound $\mathbf{3 q}$

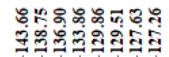

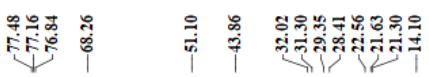

$\overbrace{\bar{s}^{n} \mathrm{Hex}}^{N H T S}$

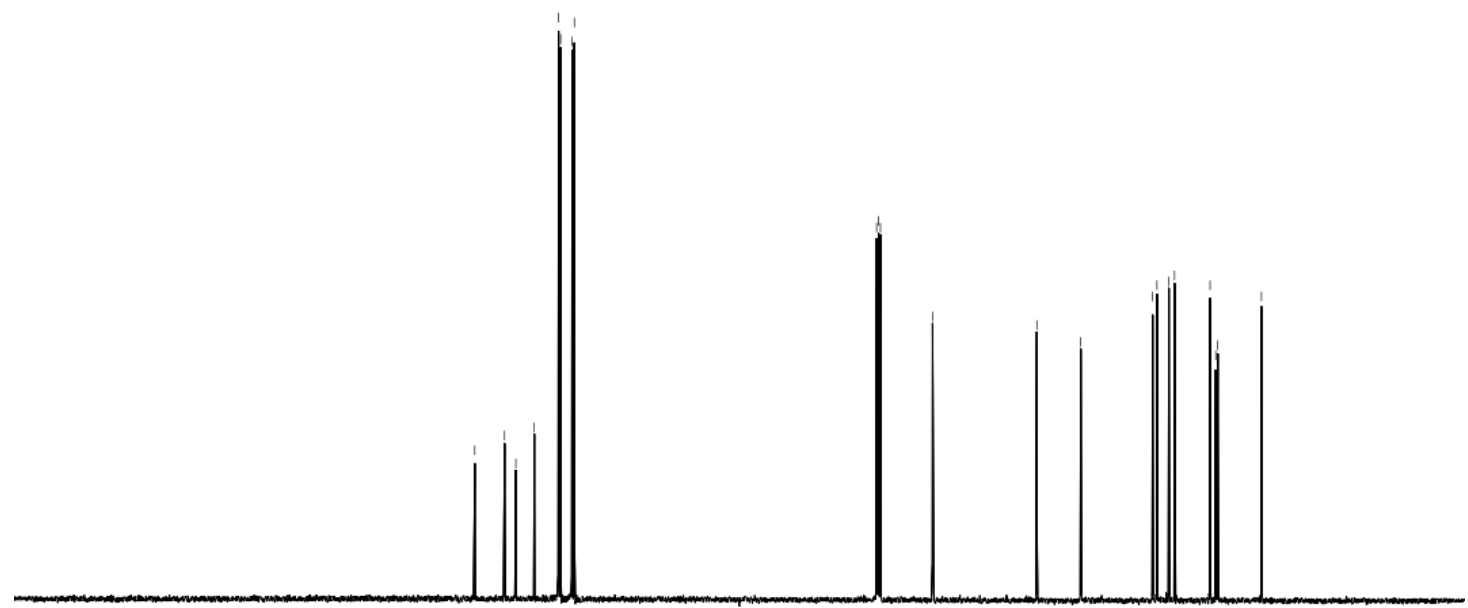

$\begin{array}{llllllllllllllllllllll}210 & 200 & 190 & 180 & 170 & 160 & 150 & 140 & 130 & 120 & 110 & 100 & 90 & 80 & 70 & 60 & 50 & 40 & 30 & 20 & 10 & 0\end{array}$ 
${ }^{1} \mathrm{H}$ NMR (400 MHz, $\left.\mathrm{CDCl}_{3}\right)$ spectrum of compound $\mathbf{3 r}$

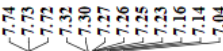

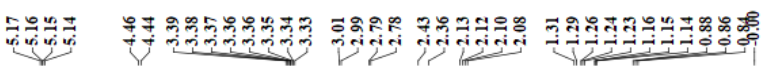
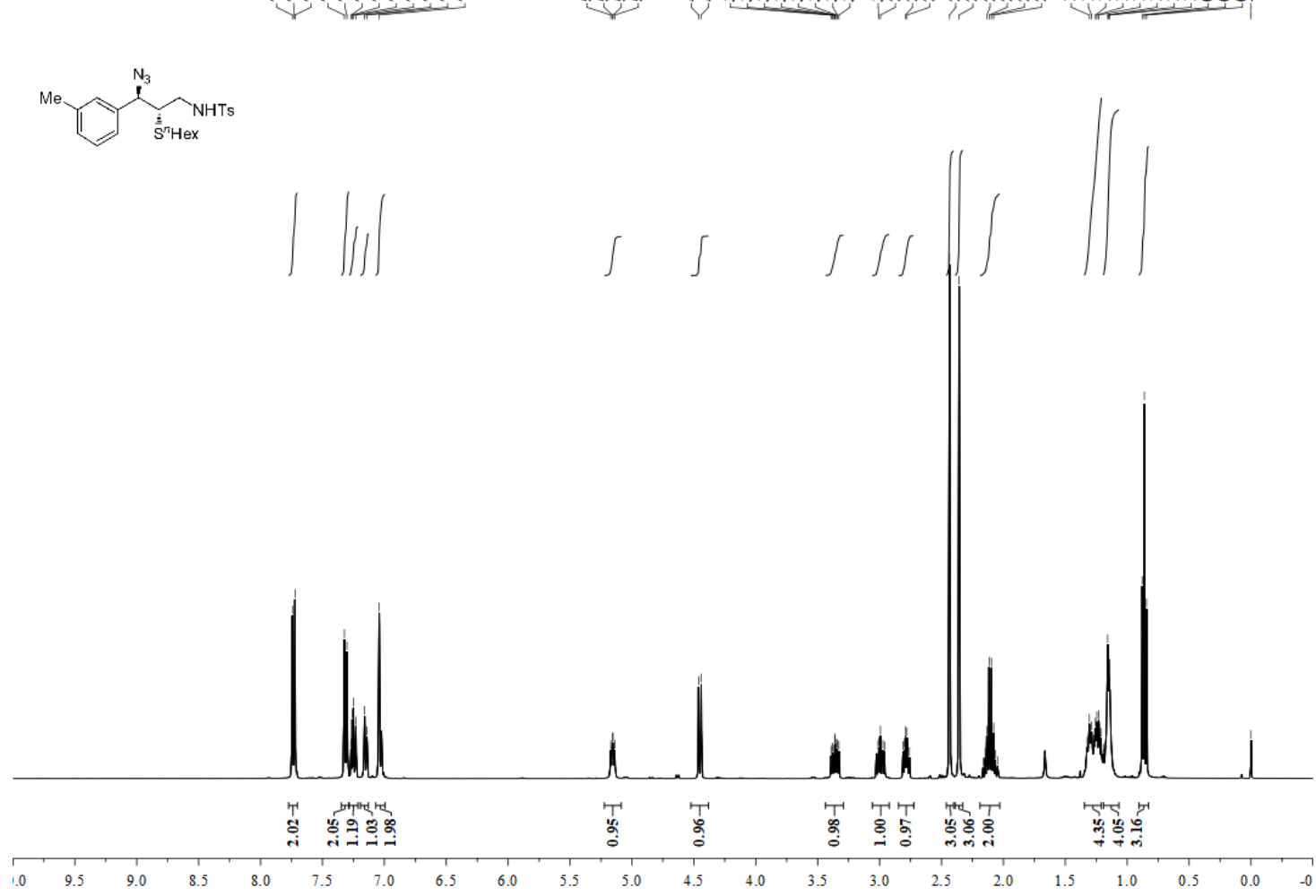

${ }^{13} \mathrm{C}$ NMR $\left(101 \mathrm{MHz}, \mathrm{CDCl}_{3}\right)$ spectrum of compound $\mathbf{3 r}$
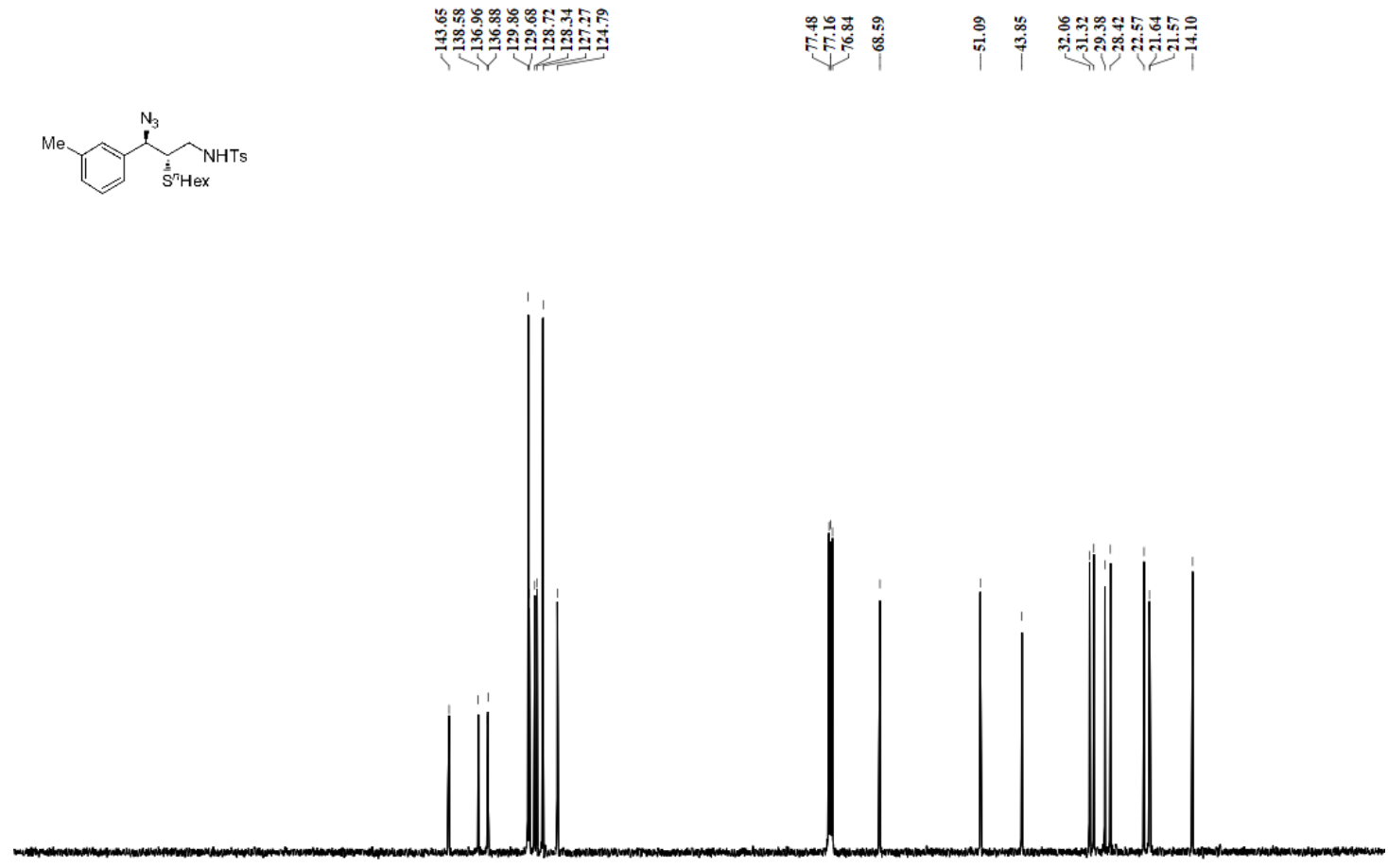

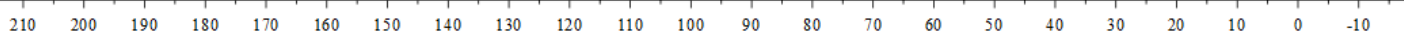


${ }^{1} \mathrm{H}$ NMR (400 MHz, $\mathrm{CDCl}_{3}$ ) spectrum of compound $\mathbf{3 s}$
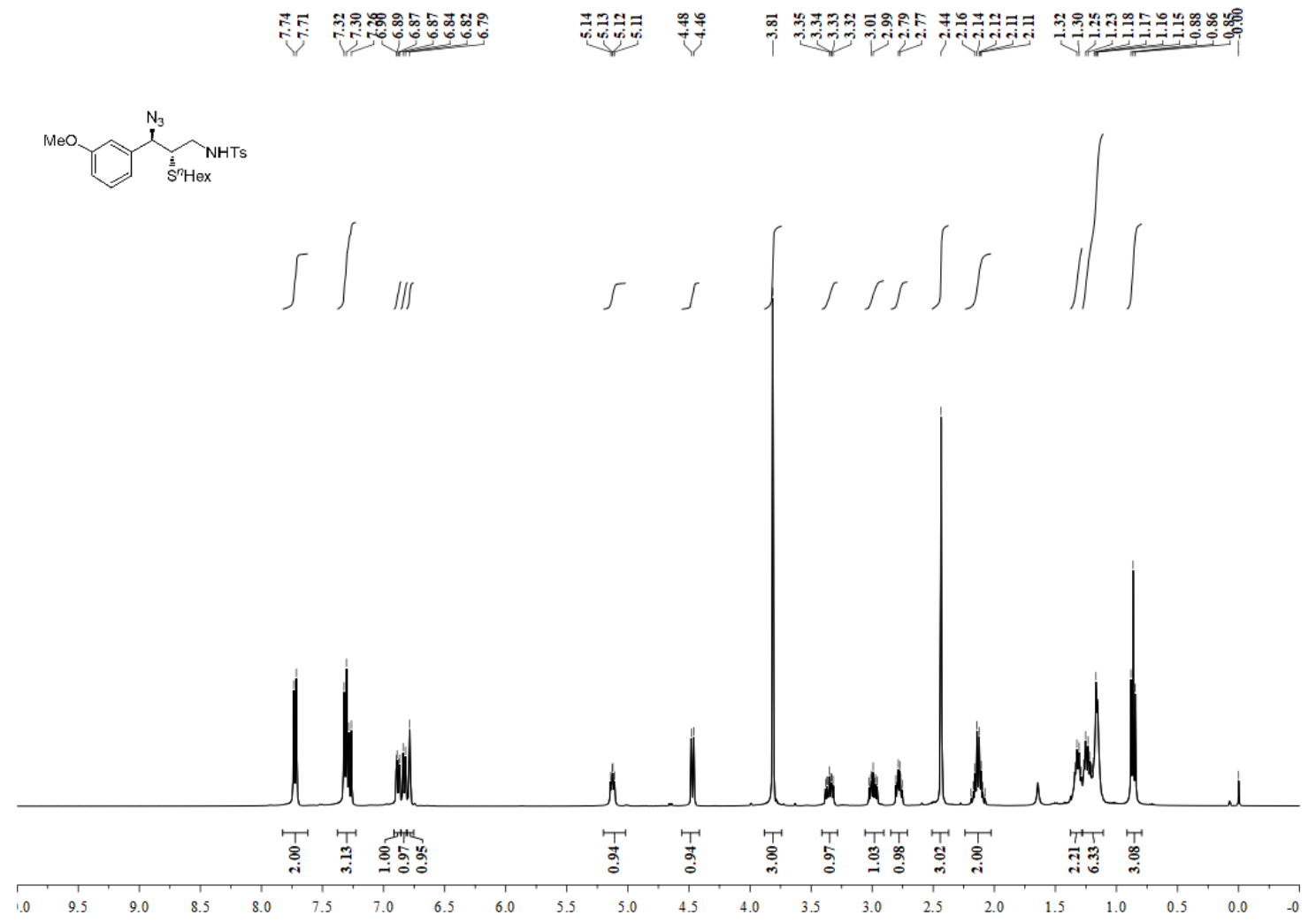

${ }^{13} \mathrm{C}$ NMR (101 MHz, $\mathrm{CDCl}_{3}$ ) spectrum of compound $\mathbf{3 s}$

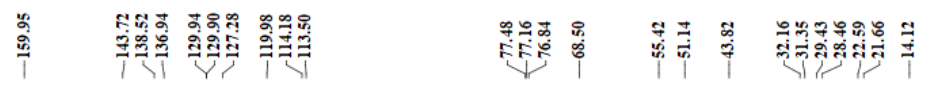<smiles>CC(C)C[C@H](N)[C@H]1CCC[C@H](O)C1</smiles>

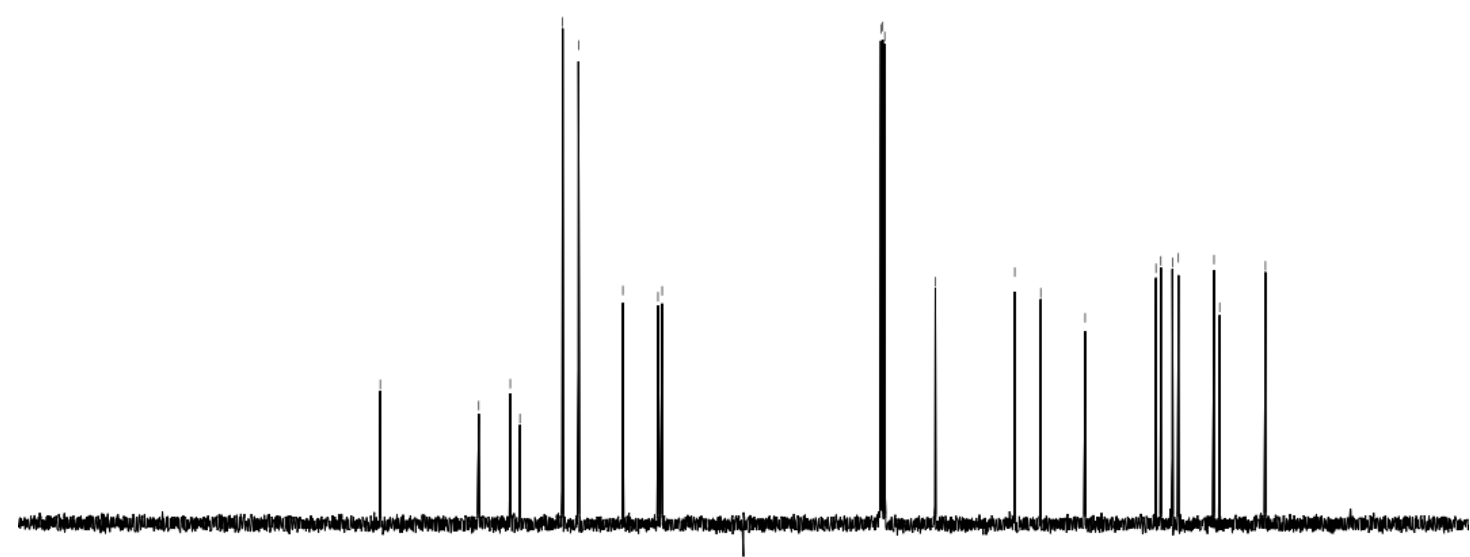

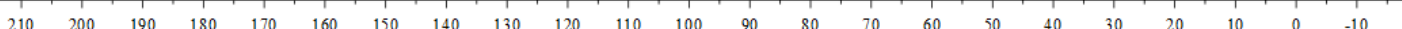


${ }^{1} \mathrm{H}$ NMR (400 MHz, $\mathrm{CDCl}_{3}$ ) spectrum of compound $\mathbf{3 t}$

ำ

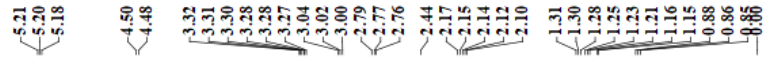

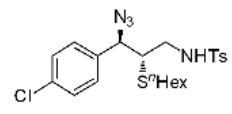

$\iiint$
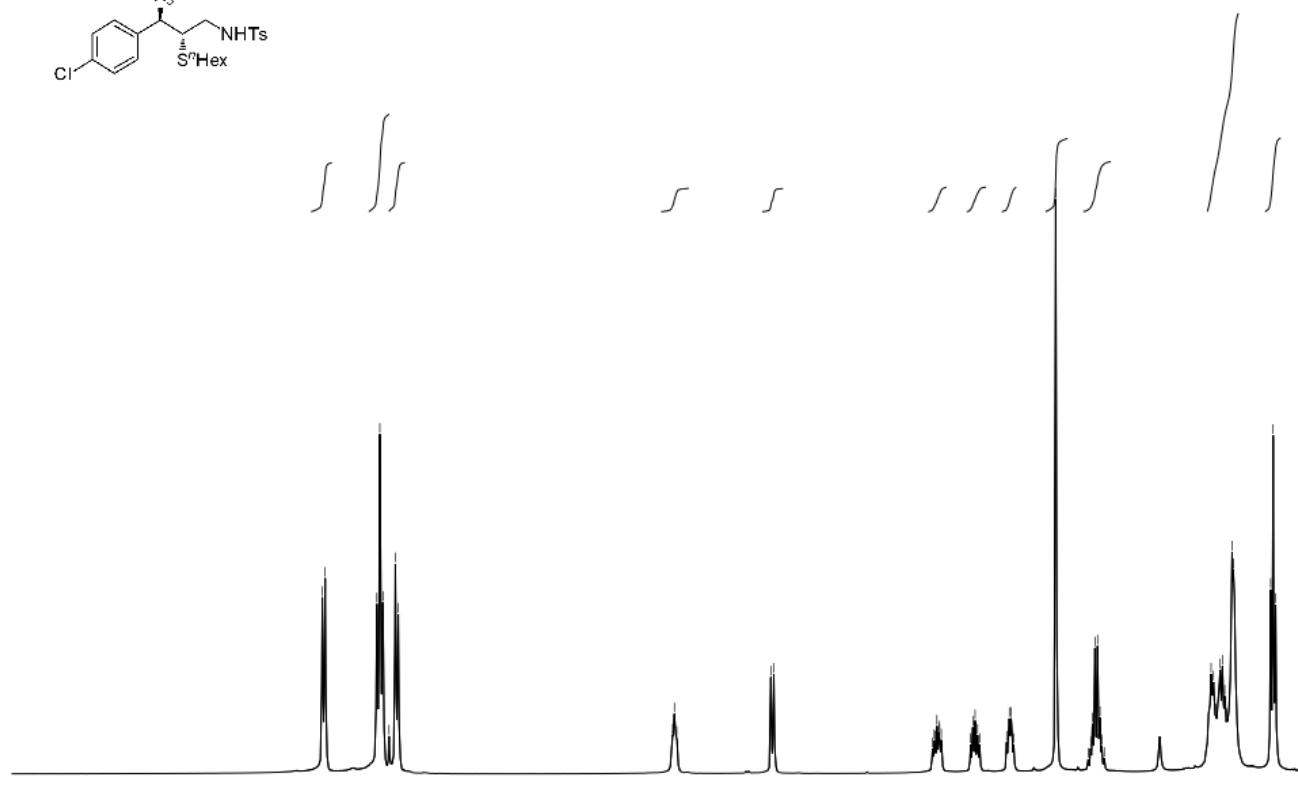

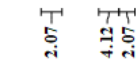

Th
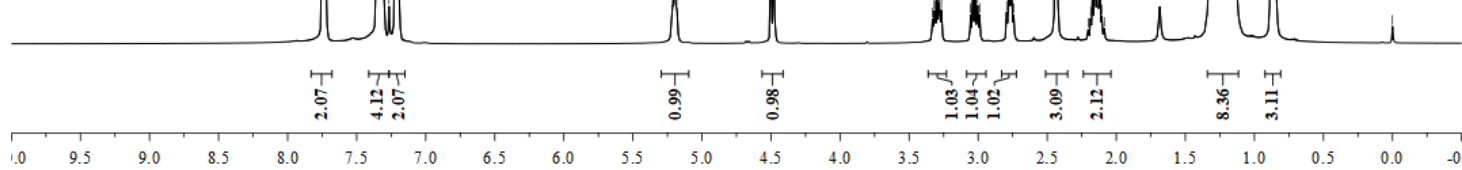

${ }^{13} \mathrm{C}$ NMR (101 MHz, $\mathrm{CDCl}_{3}$ ) spectrum of compound 3t

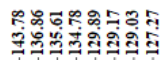

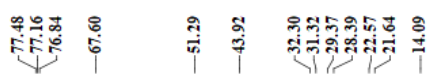
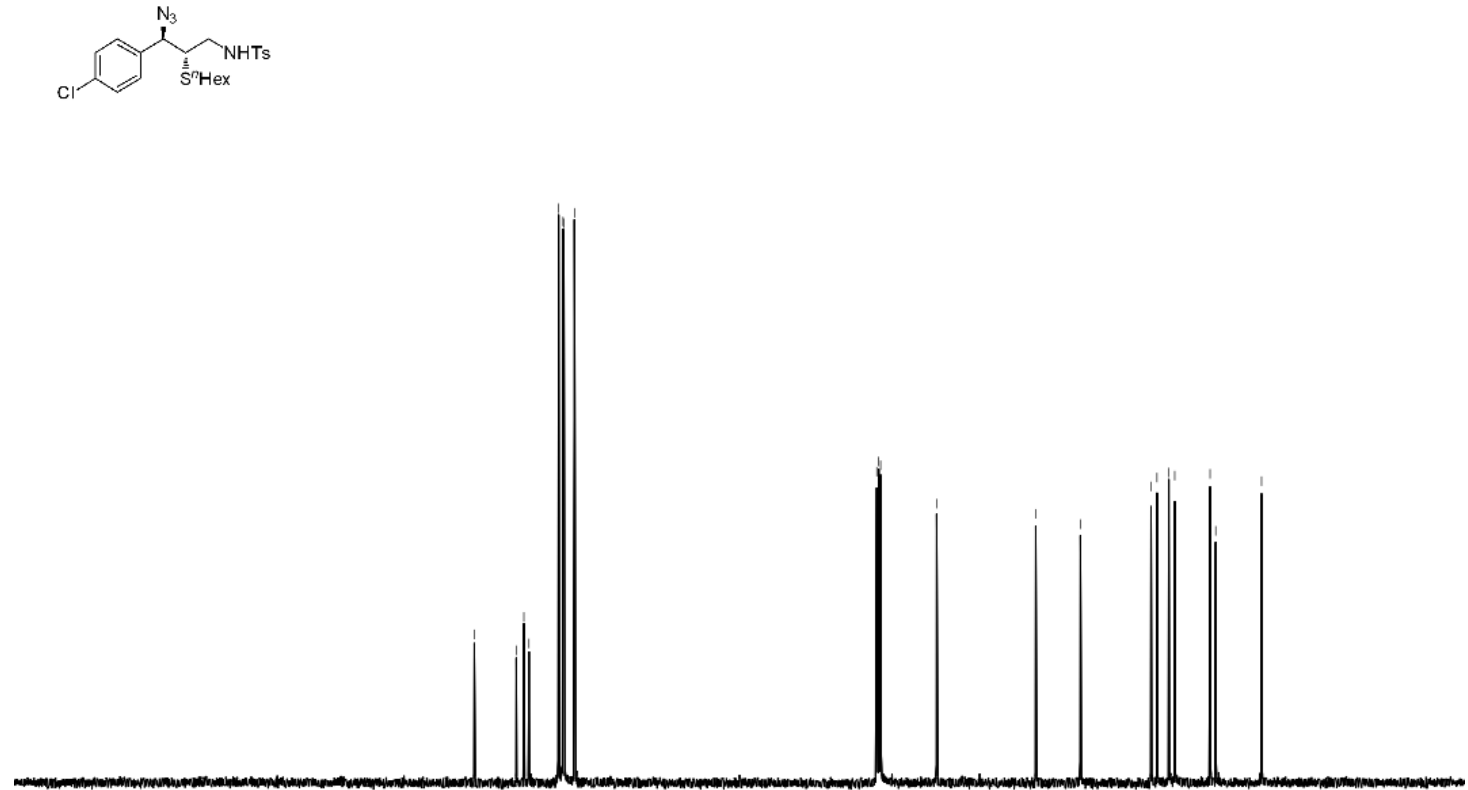

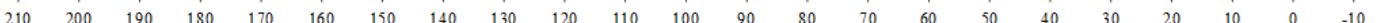


${ }^{1} \mathrm{H}$ NMR (400 MHz, $\mathrm{CDCl}_{3}$ ) spectrum of compound 3u

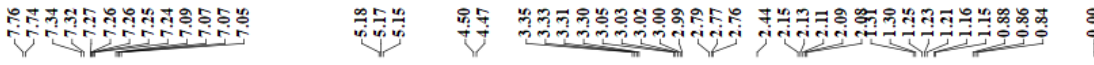

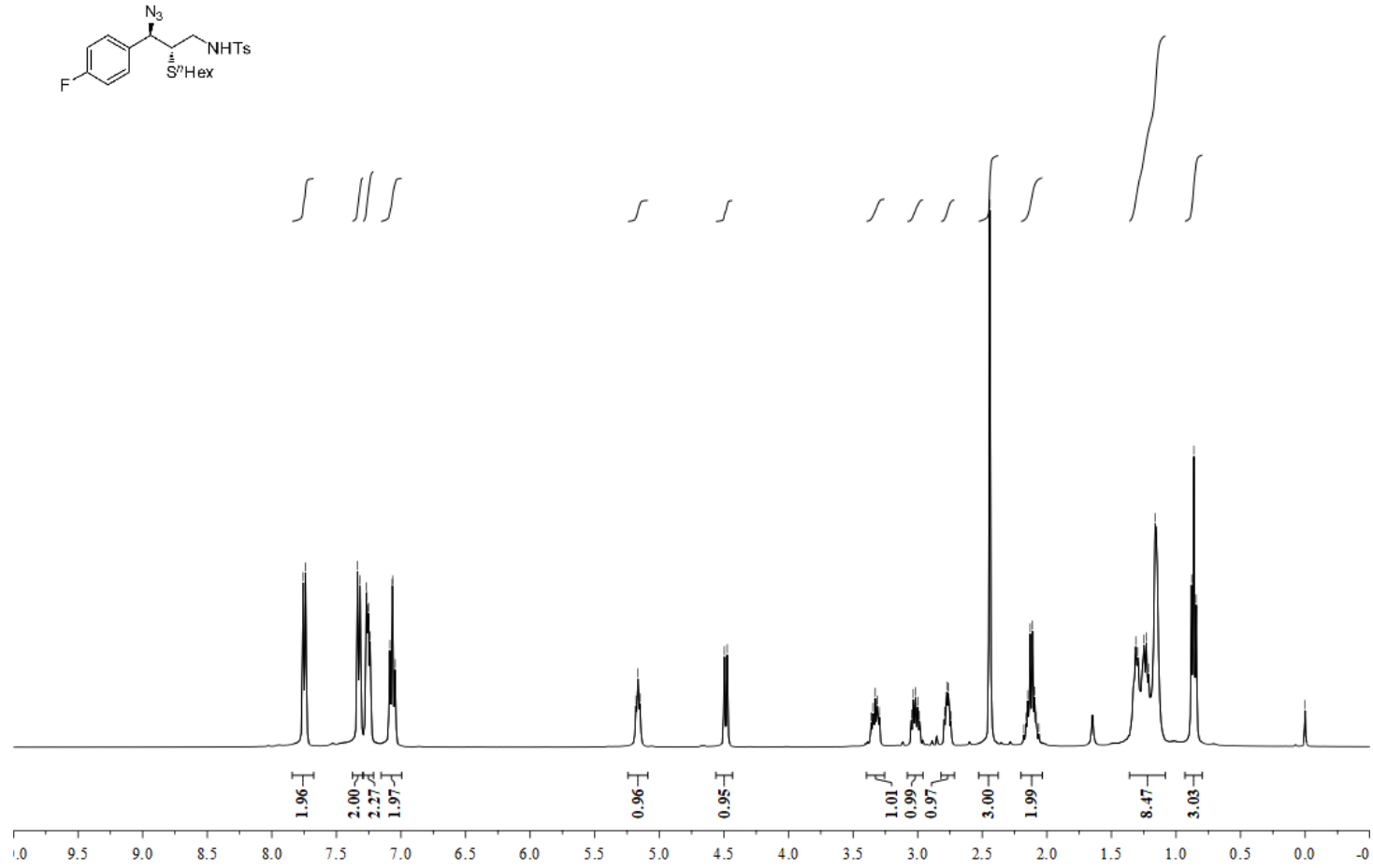

${ }^{13} \mathrm{C}$ NMR (101 MHz, $\left.\mathrm{CDCl}_{3}\right)$ spectrum of compound $\mathbf{3 u}$

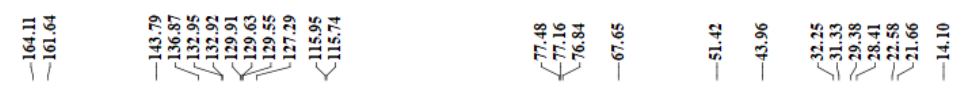<smiles>CCCC(N)C1CCCC1</smiles>

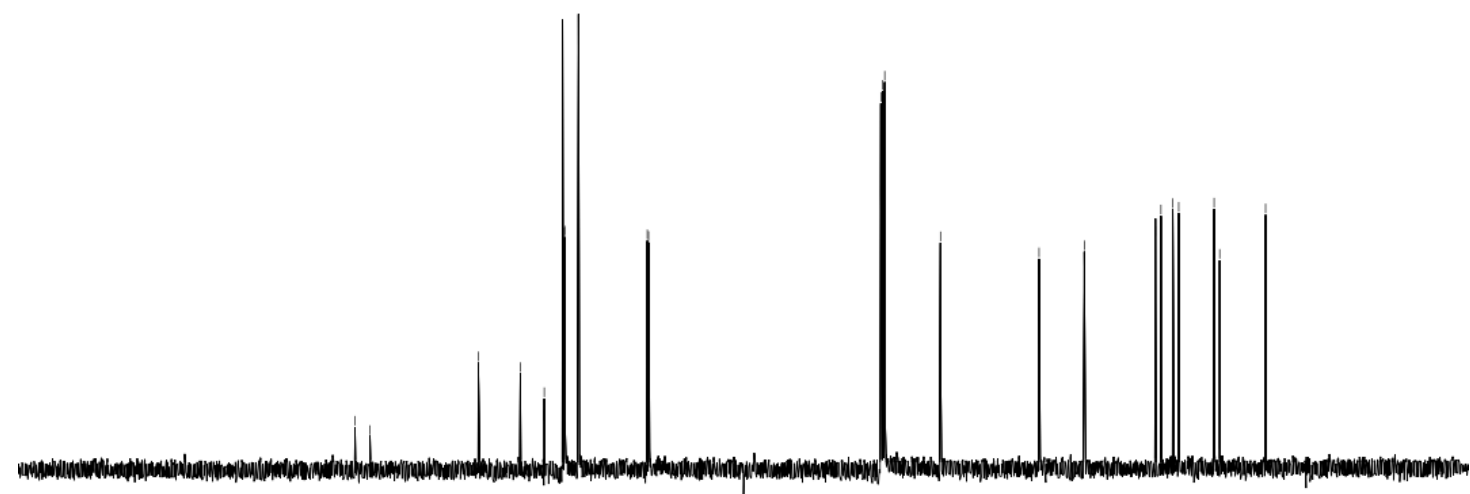

$\begin{array}{lllllllllllllllllllllllllllllllllll}210 & 200 & 190 & 180 & 170 & 160 & 150 & 140 & 130 & 120 & 110 & 100 & 90 & 80 & 70 & 60 & 50 & 40 & 30 & 20 & 10 & 0\end{array}$ 
${ }^{1} \mathrm{H}$ NMR (400 MHz, $\mathrm{CDCl}_{3}$ ) spectrum of compound $\mathbf{3 v}$

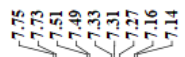

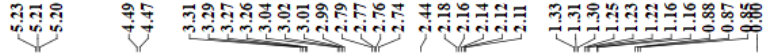

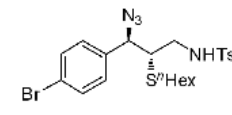<smiles>C1#CCCCC1</smiles>

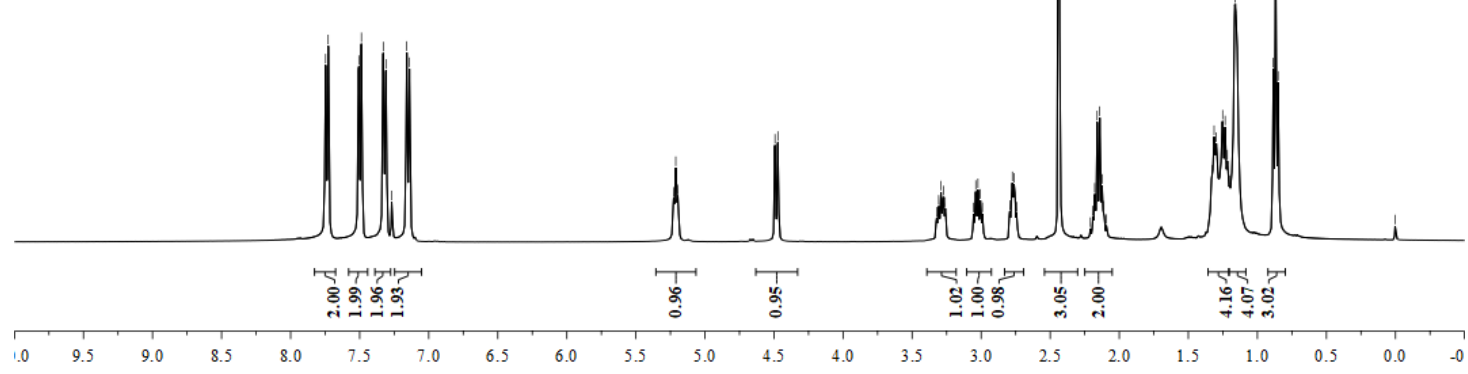

${ }^{13} \mathrm{C}$ NMR (101 MHz, $\mathrm{CDCl}_{3}$ ) spectrum of compound $\mathbf{3 v}$

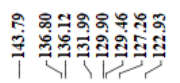

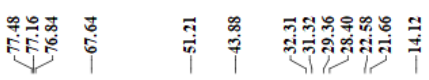

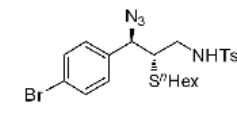

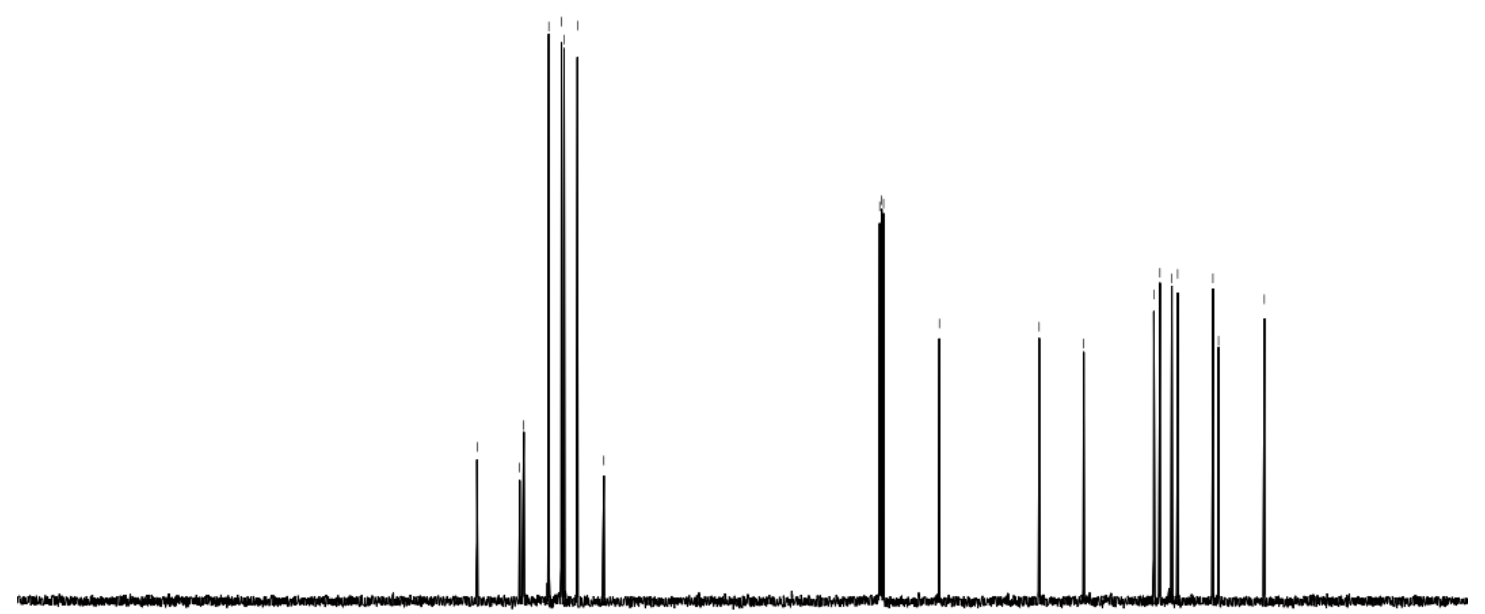

$\begin{array}{llllllllllllllllllllll}210 & 200 & 190 & 180 & 170 & 160 & 150 & 140 & 130 & 120 & 110 & 100 & 90 & 80 & 70 & 60 & 50 & 40 & 30 & 20 & 10 & 0\end{array}$ 
${ }^{1} \mathrm{H}$ NMR (400 MHz, $\mathrm{CDCl}_{3}$ ) spectrum of compound $\mathbf{3 w}$

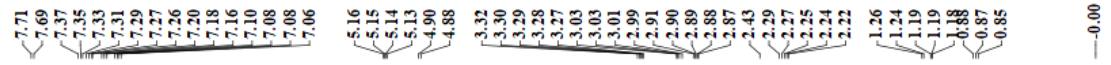<smiles>CCCCC(N)C1CCC=C1C</smiles><smiles>CC1CCCCC1</smiles>

\section{$\iint$}
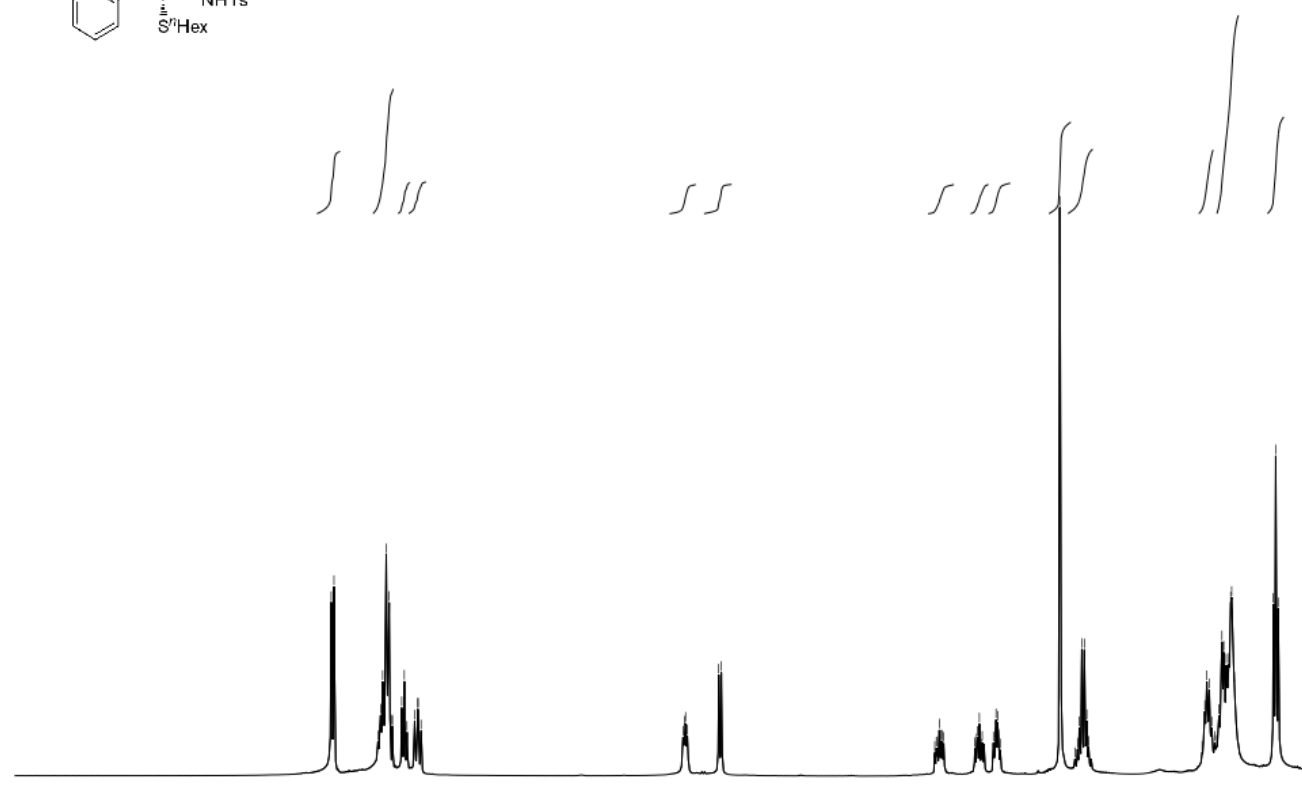

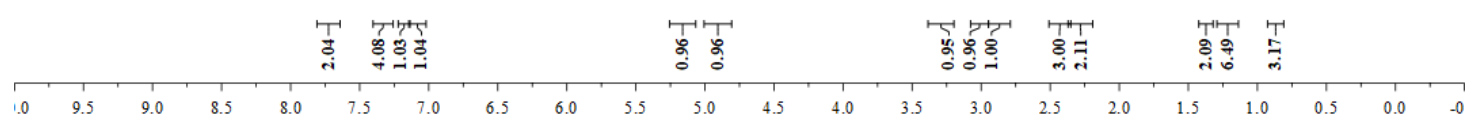

${ }^{13} \mathrm{C}$ NMR (101 MHz, $\left.\mathrm{CDCl}_{3}\right)$ spectrum of compound $\mathbf{3 w}$

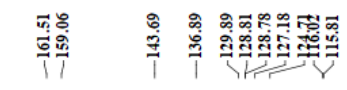

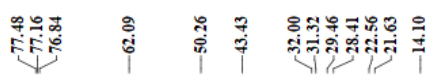
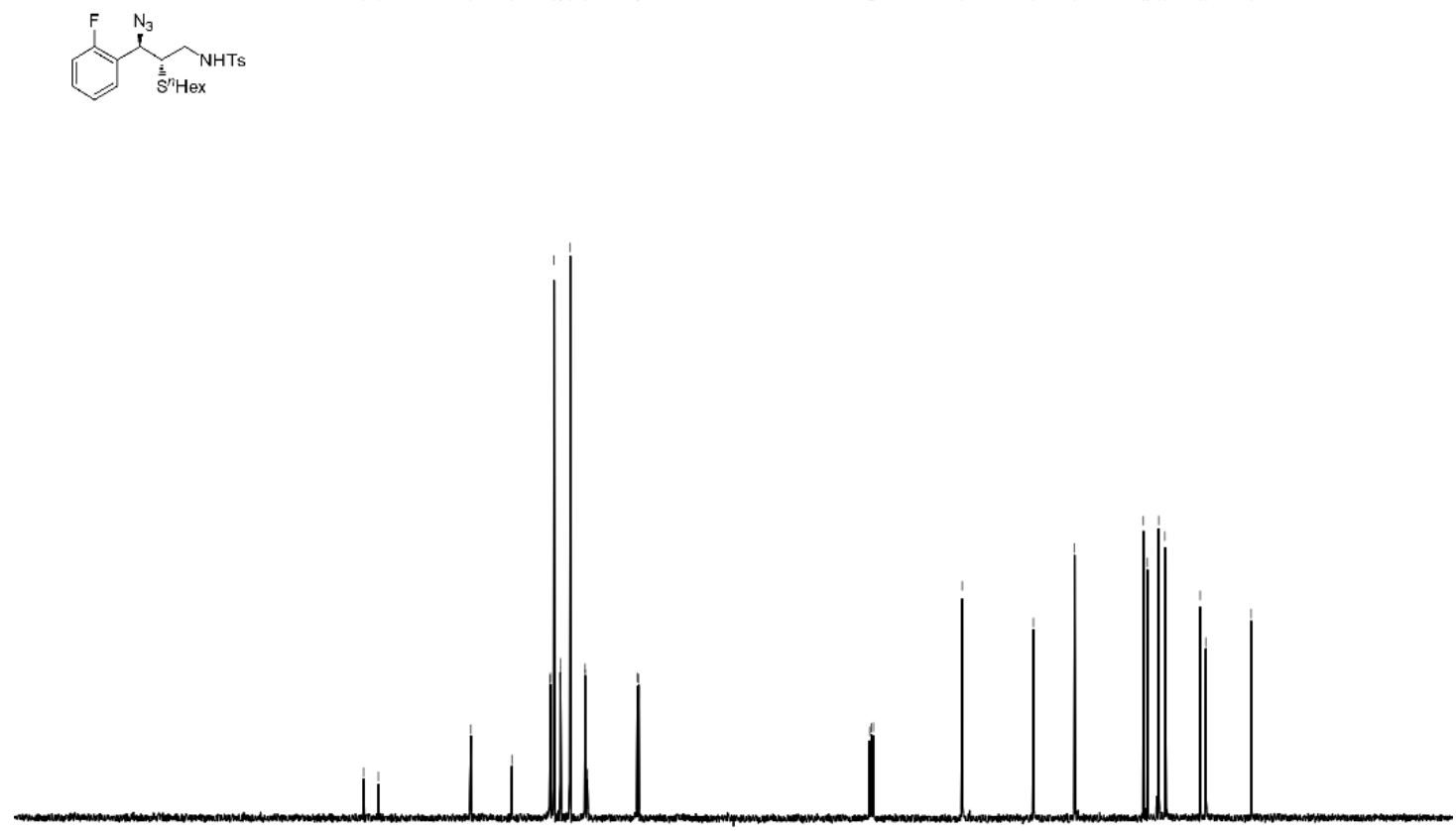

$\begin{array}{llllllllllllllllllllll}210 & 200 & 190 & 180 & 170 & 160 & 150 & 140 & 130 & 120 & 110 & 100 & 90 & 80 & 70 & 60 & 50 & 40 & 30 & 20 & 10 & 0\end{array}$ 
${ }^{1} \mathrm{H}$ NMR (400 MHz, $\mathrm{CDCl}_{3}$ ) spectrum of compound 3x

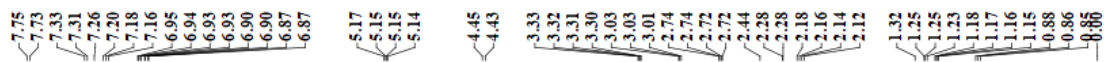

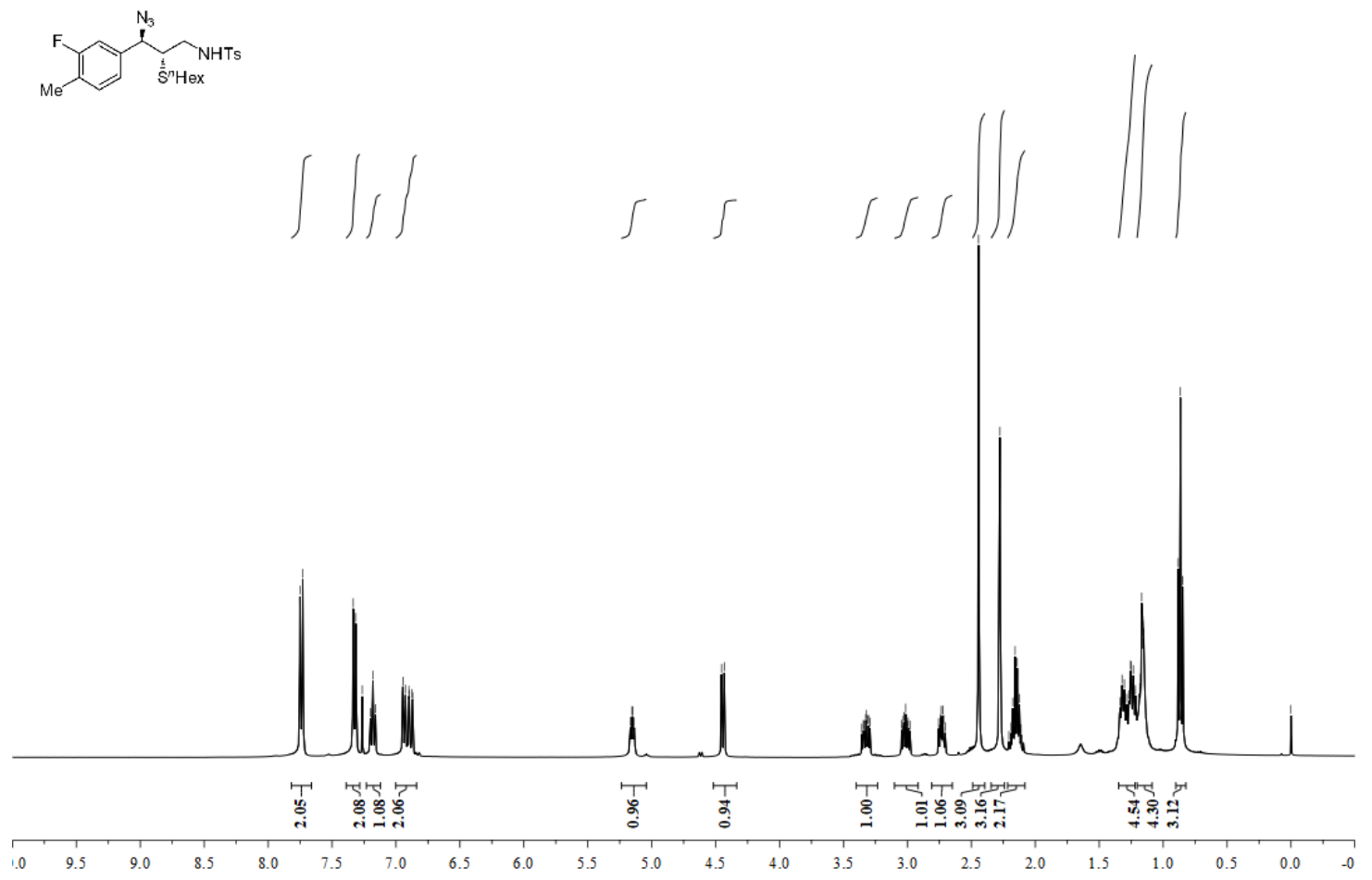

${ }^{13} \mathrm{C}$ NMR (101 MHz, $\left.\mathrm{CDCl}_{3}\right)$ spectrum of compound $\mathbf{3 x}$

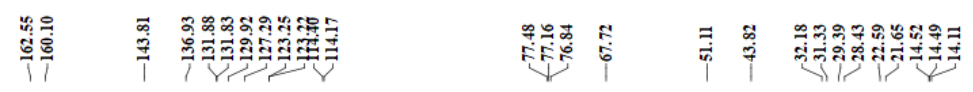
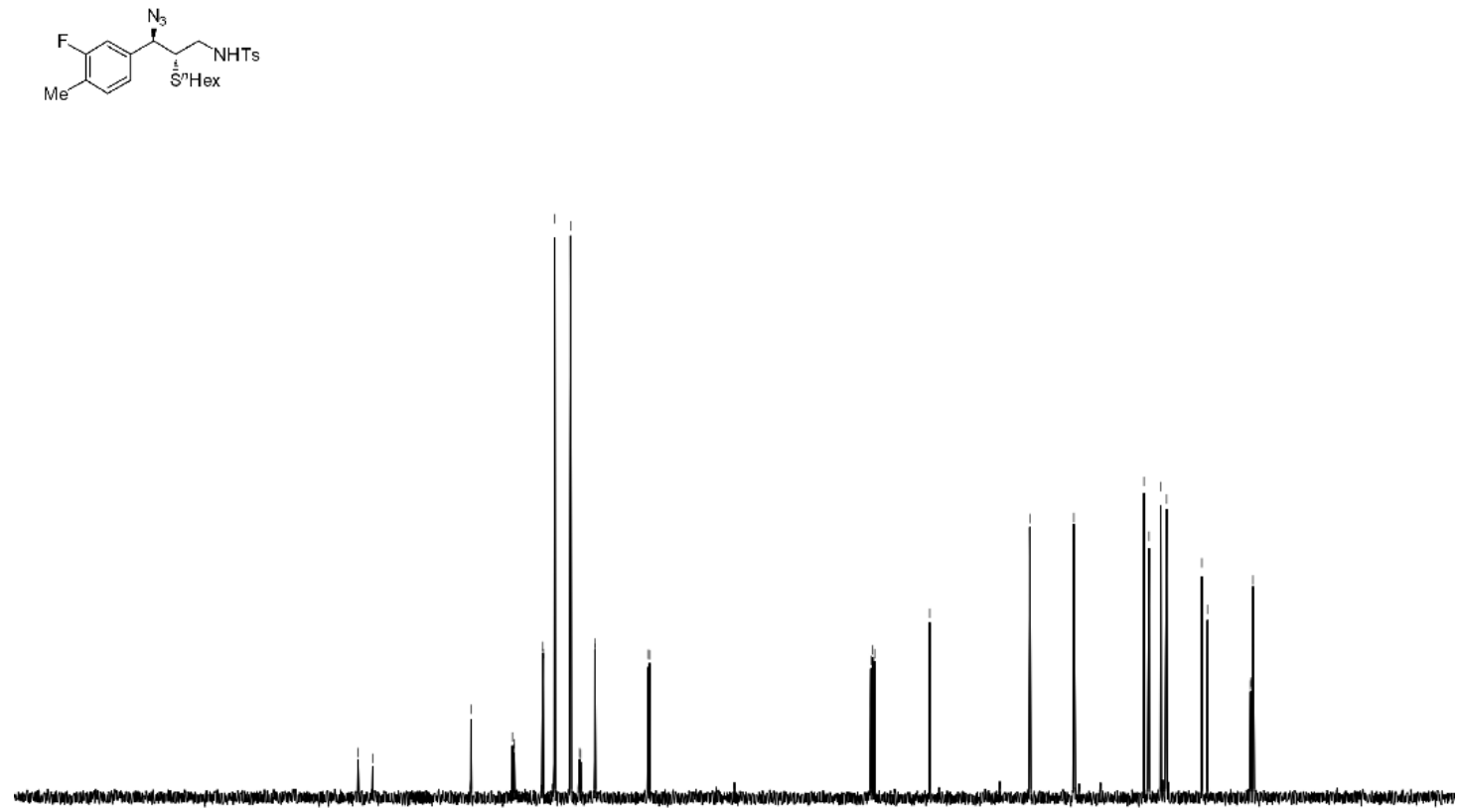

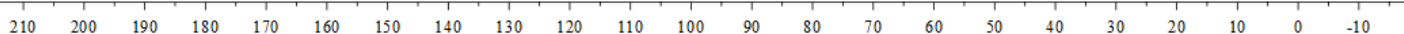


${ }^{1} \mathrm{H}$ NMR (400 MHz, $\mathrm{CDCl}_{3}$ ) spectrum of compound $\mathbf{3 y}$

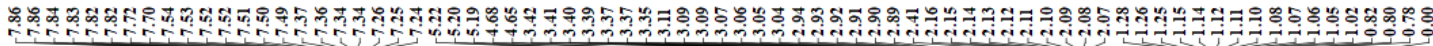
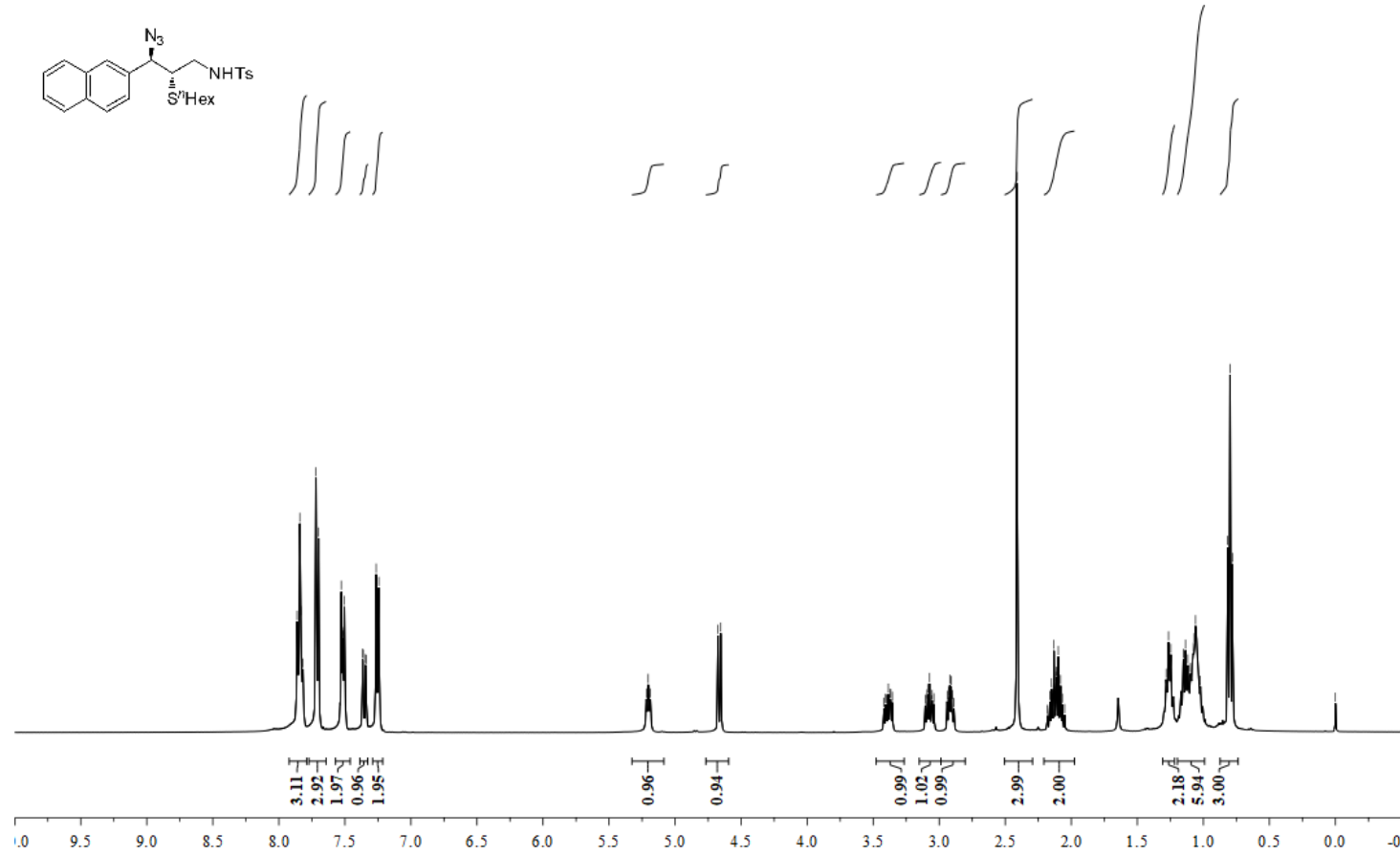

${ }^{13} \mathrm{C}$ NMR (101 MHz, $\mathrm{CDCl}_{3}$ ) spectrum of compound $\mathbf{3 y}$

\begin{tabular}{|c|c|}
\hline 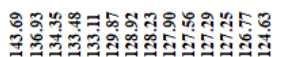 & 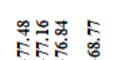 \\
\hline
\end{tabular}
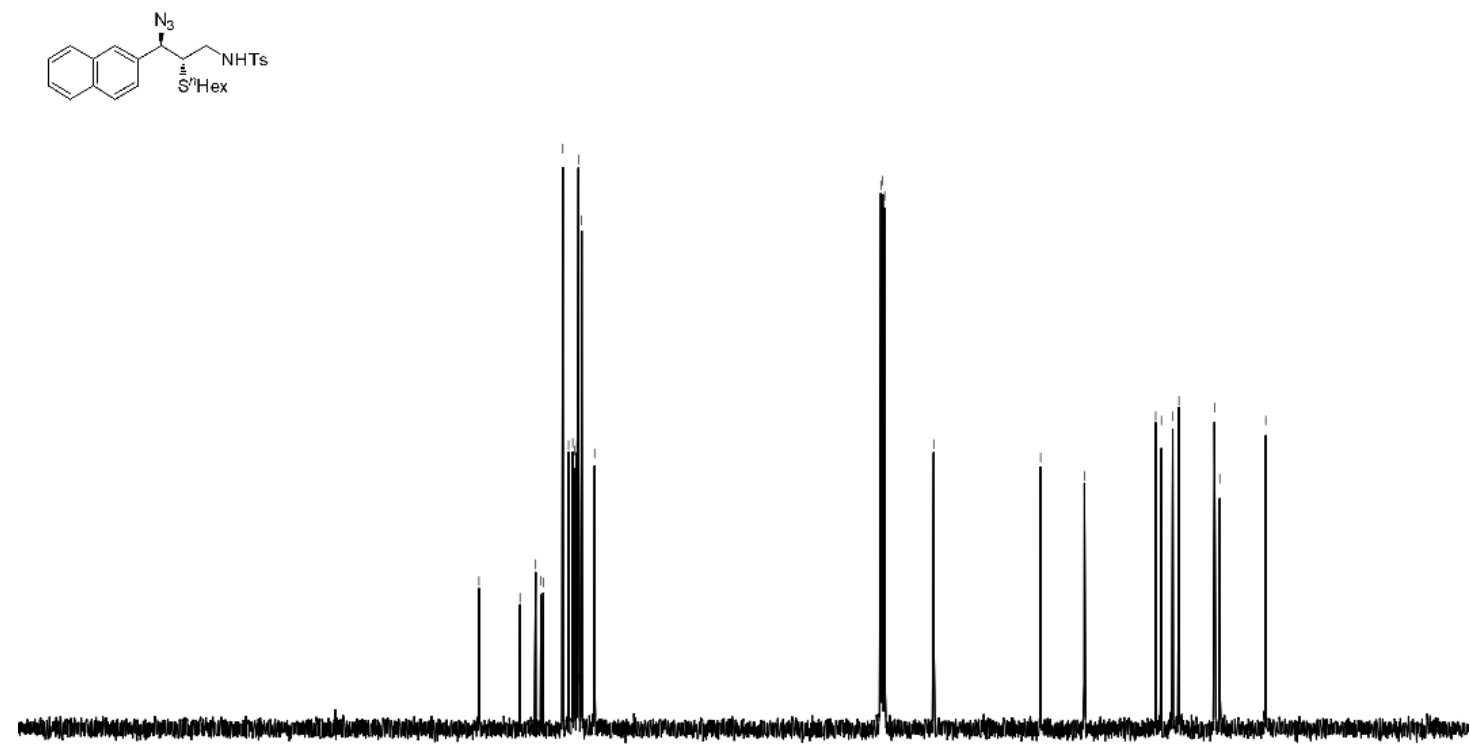

$\begin{array}{lllllllllllllllllllllll}210 & 200 & 190 & 180 & 170 & 160 & 150 & 140 & 130 & 120 & 110 & 100 & 90 & 80 & 70 & 60 & 50 & 40 & 30 & 20 & 10 & 0\end{array}$ 
${ }^{1} \mathrm{H}$ NMR (400 MHz, $\mathrm{CDCl}_{3}$ ) spectrum of compound $\mathbf{3 z}$

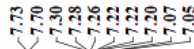

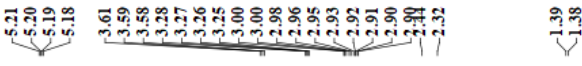

$\stackrel{8}{8}$

(NHTs
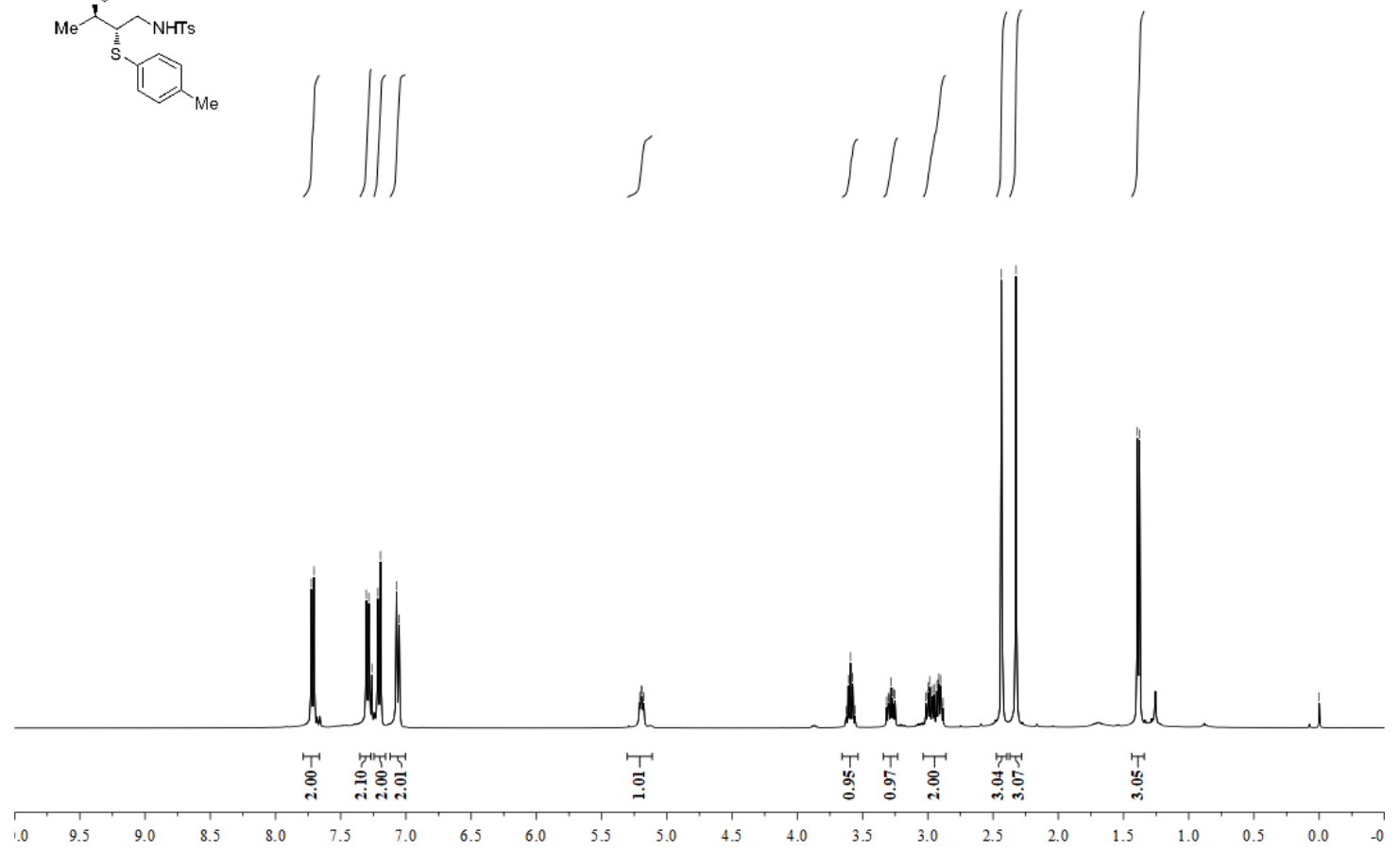

${ }^{13} \mathrm{C}$ NMR $\left(101 \mathrm{MHz}, \mathrm{CDCl}_{3}\right)$ spectrum of compound $\mathbf{3 z}$

\begin{tabular}{|c|c|}
\hline 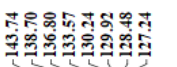 & 是是营 \\
\hline
\end{tabular}

$\overbrace{\text { NHTs }}^{N_{3}}$

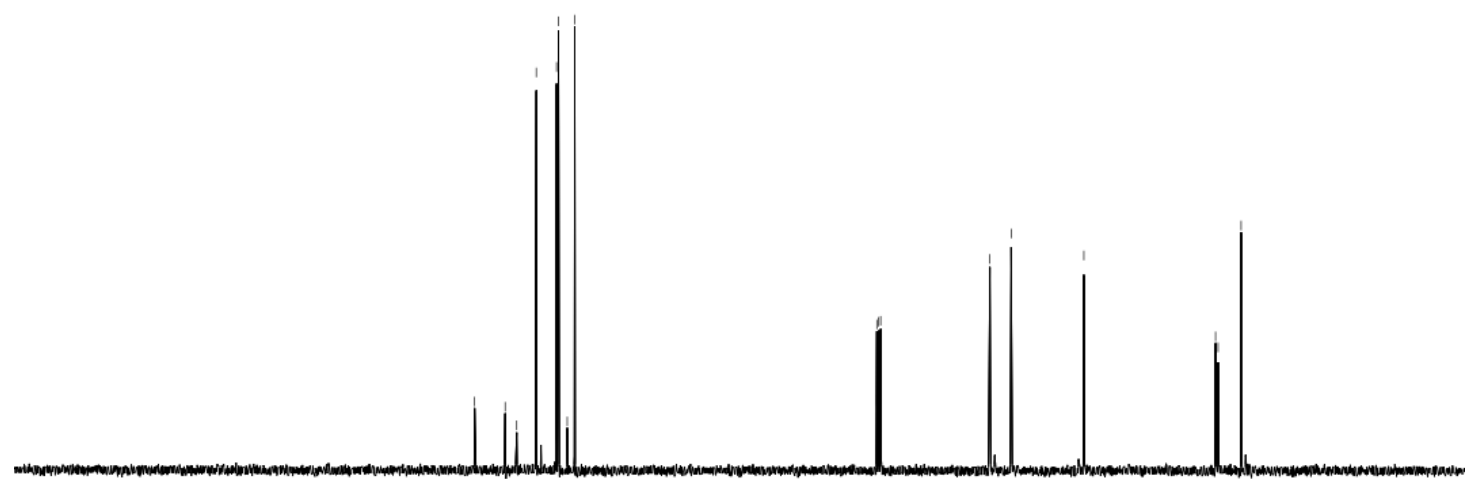

$\begin{array}{llllllllllllllllllllllll}210 & 200 & 190 & 180 & 170 & 160 & 150 & 140 & 130 & 120 & 110 & 100 & 90 & 80 & 70 & 60 & 50 & 40 & 30 & 20 & 10 & 0\end{array}$ 
${ }^{1} \mathrm{H}$ NMR (400 MHz, $\mathrm{CDCl}_{3}$ ) spectrum of compound 3aa

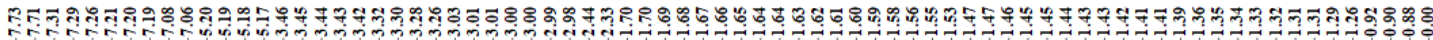

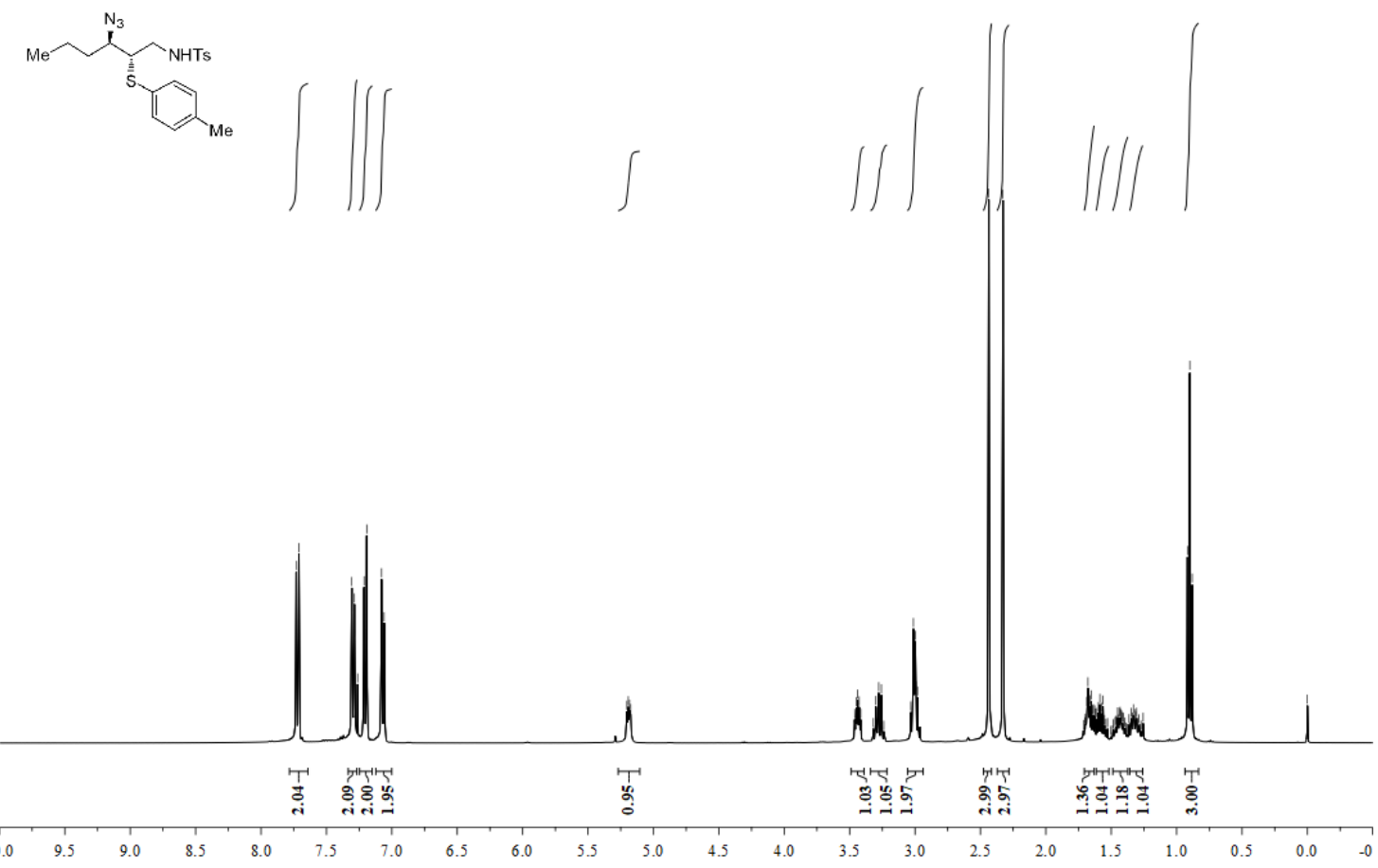

${ }^{13} \mathrm{C}$ NMR $\left(101 \mathrm{MHz}, \mathrm{CDCl}_{3}\right)$ spectrum of compound 3aa

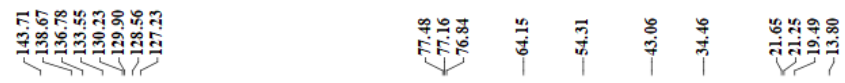

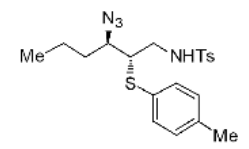

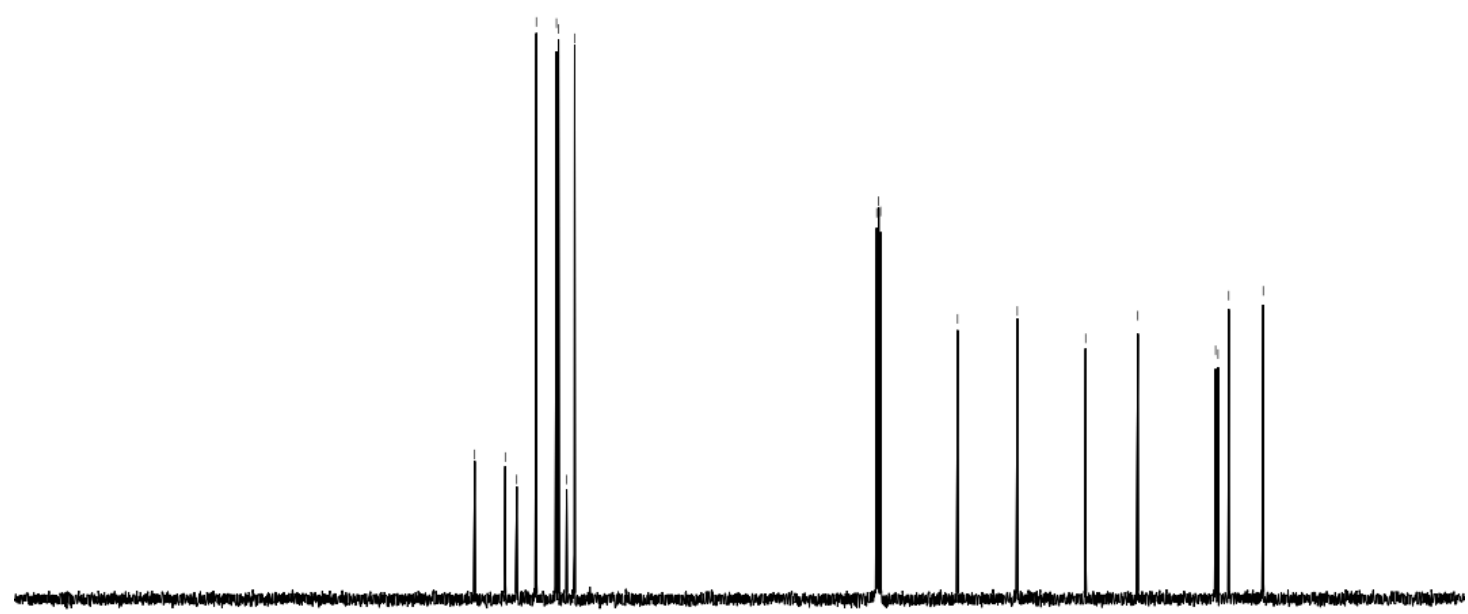

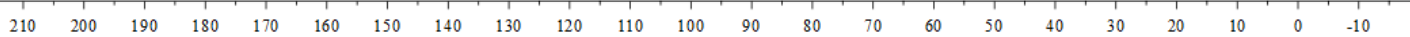


${ }^{1} \mathrm{H}$ NMR (400 MHz, $\mathrm{CDCl}_{3}$ ) spectrum of compound 3ab

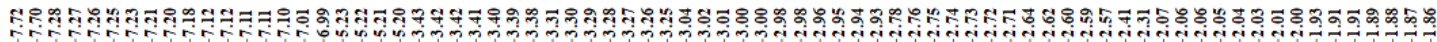
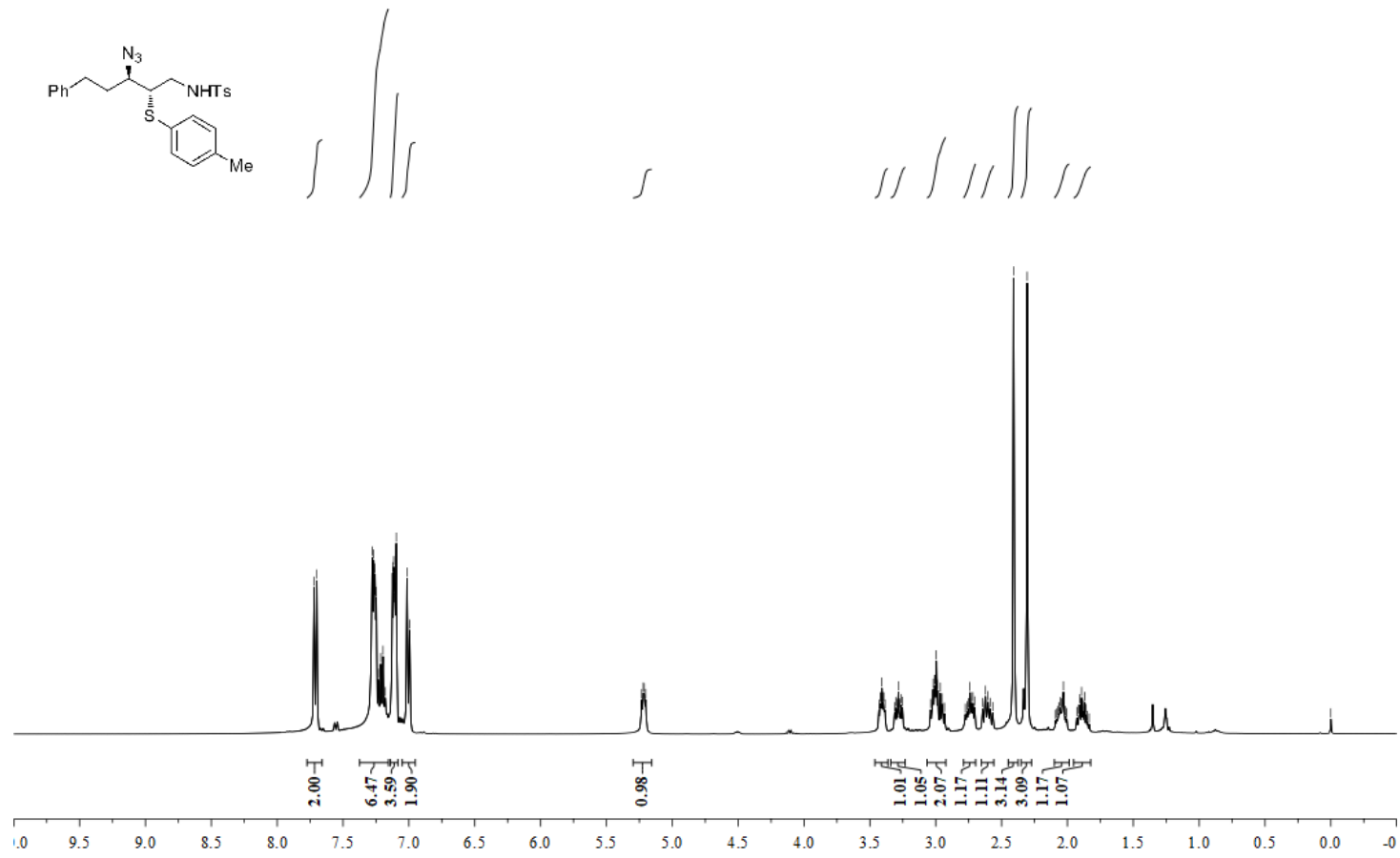

${ }^{13} \mathrm{C}$ NMR $\left(101 \mathrm{MHz}, \mathrm{CDCl}_{3}\right)$ spectrum of compound 3ab

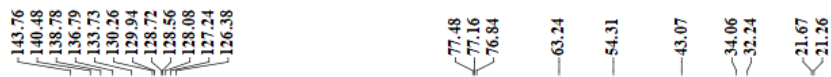

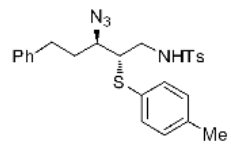

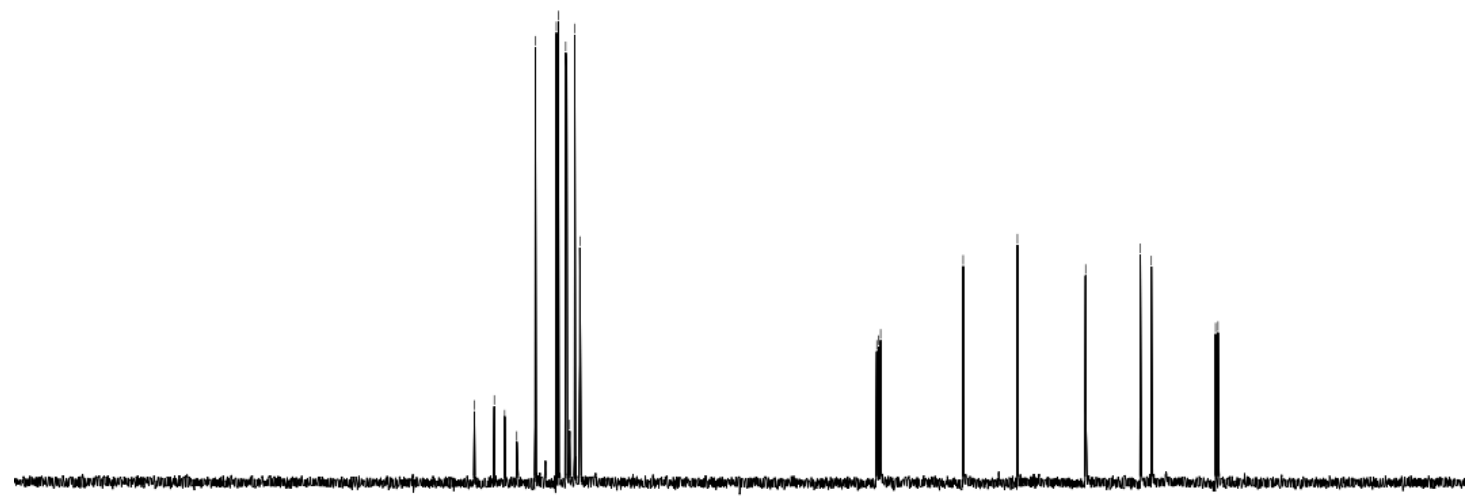

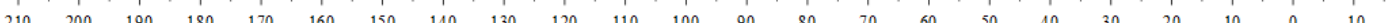


${ }^{1} \mathrm{H}$ NMR (400 MHz, $\mathrm{CDCl}_{3}$ ) spectrum of compound 4a gำ

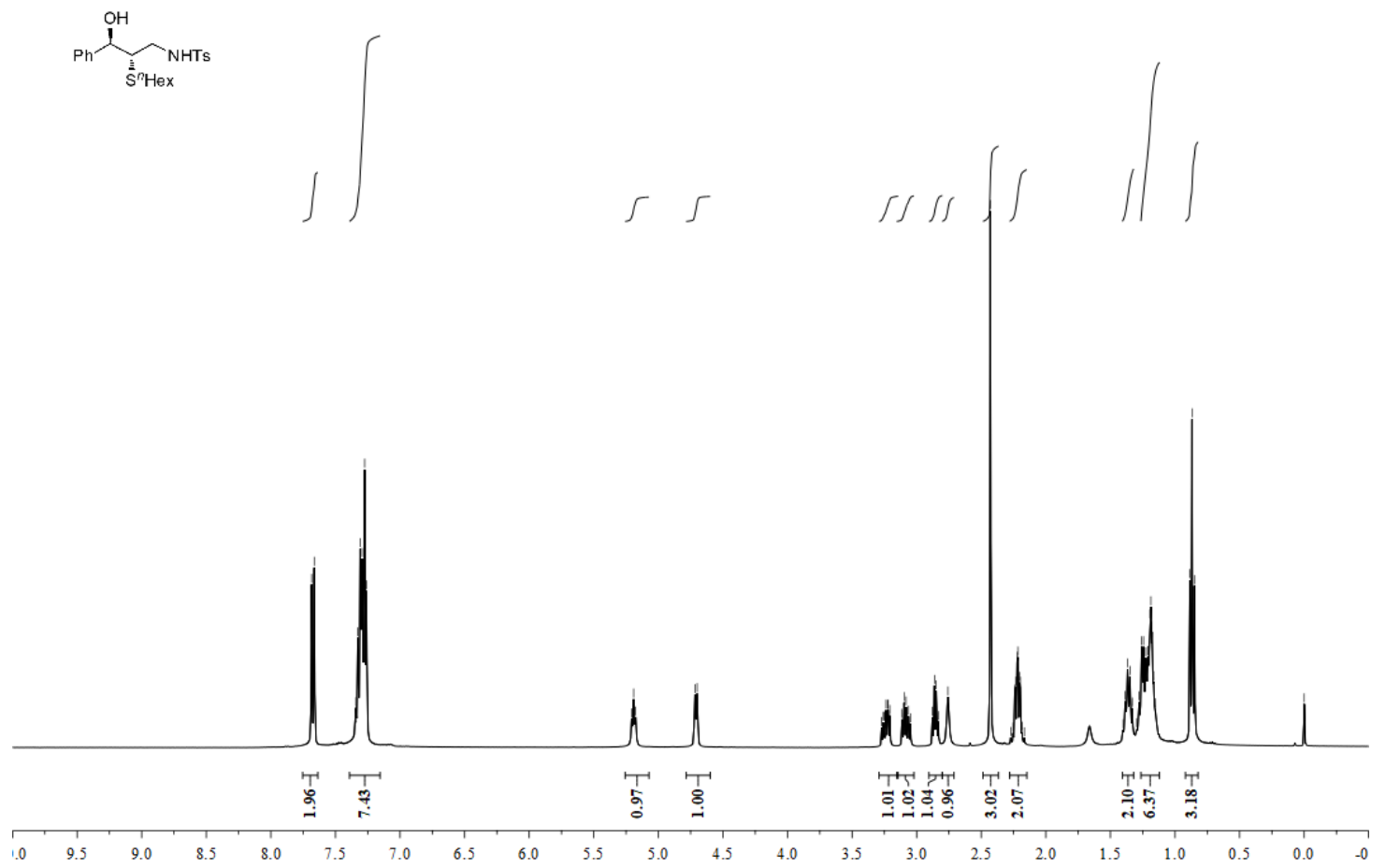

${ }^{13} \mathrm{C}$ NMR $\left(101 \mathrm{MHz}, \mathrm{CDCl}_{3}\right)$ spectrum of compound 4a

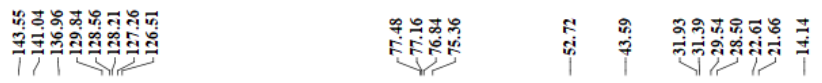
$\overbrace{\mathrm{S}^{\mathrm{N}} \mathrm{Hex}}^{\mathrm{NHTS}}$

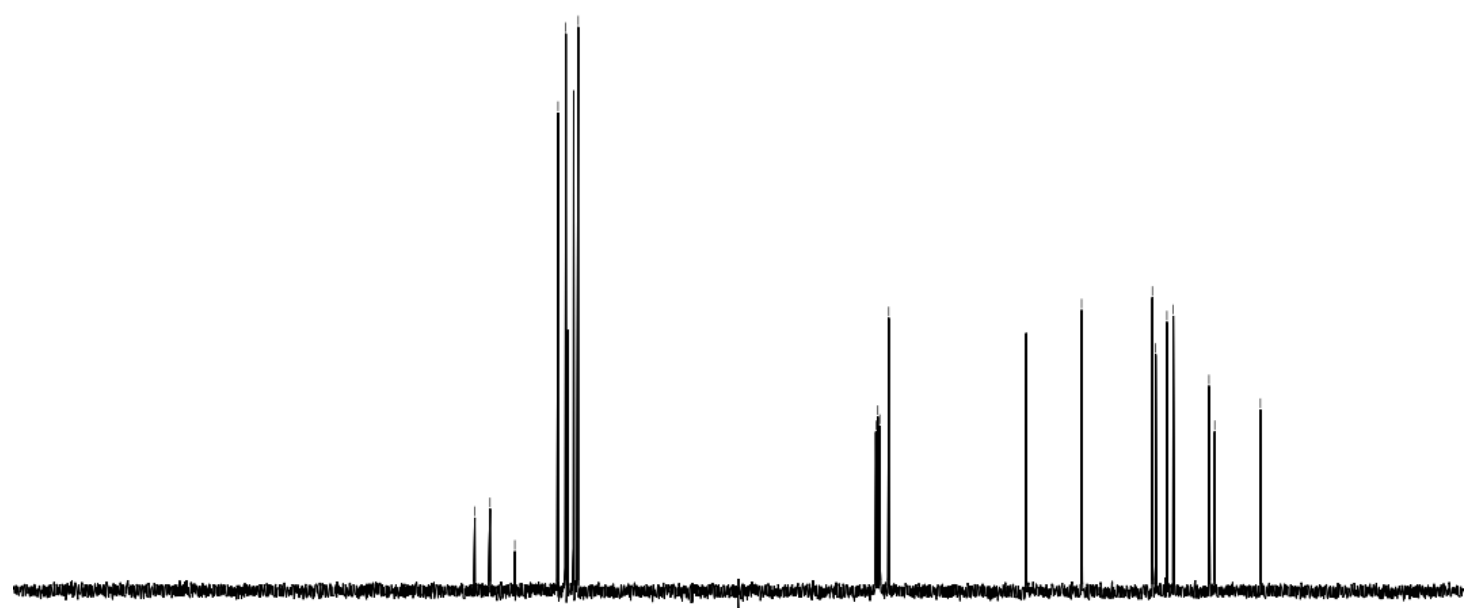

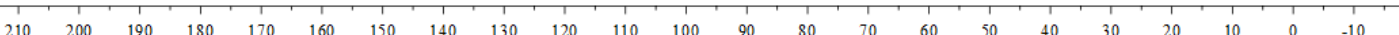


${ }^{1} \mathrm{H}$ NMR (400 MHz, $\mathrm{CDCl}_{3}$ ) spectrum of compound $\mathbf{4 b}$

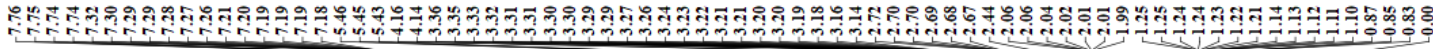

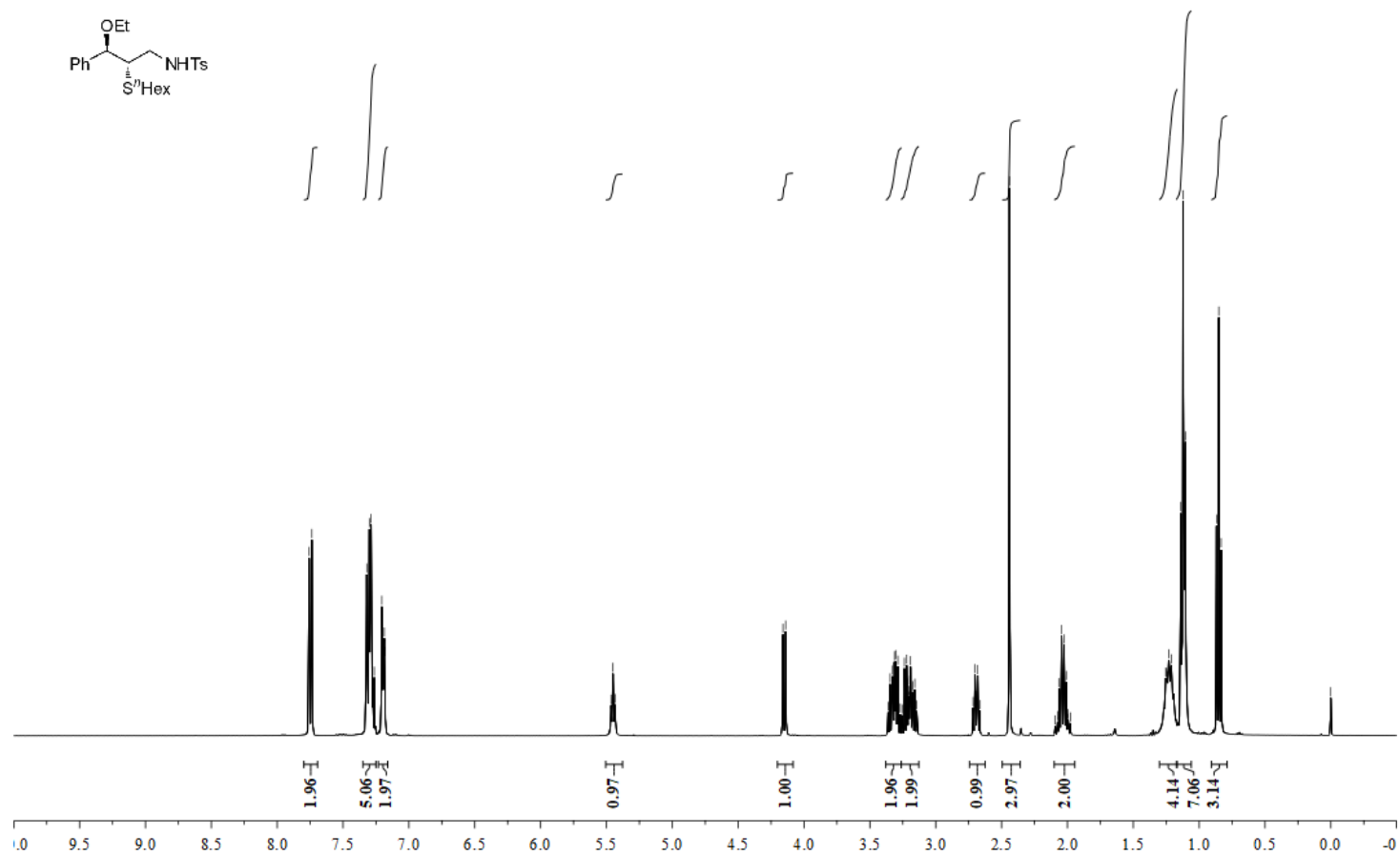

${ }^{13} \mathrm{C}$ NMR (101 MHz, $\left.\mathrm{CDCl}_{3}\right)$ spectrum of compound $\mathbf{4 b}$

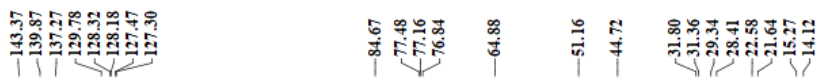

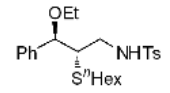

$\begin{array}{llllllllllllllllllllll}210 & 200 & 190 & 180 & 170 & 160 & 150 & 140 & 130 & 120 & 110 & 100 & 90 & 80 & 70 & 60 & 50 & 40 & 30 & 20 & 10 & 0\end{array}$ 
${ }^{1} \mathrm{H}$ NMR (400 MHz, $\left.\mathrm{CDCl}_{3}\right)$ spectrum of compound $\mathbf{4 c}$

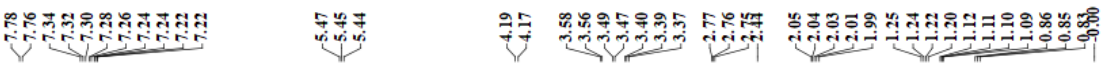

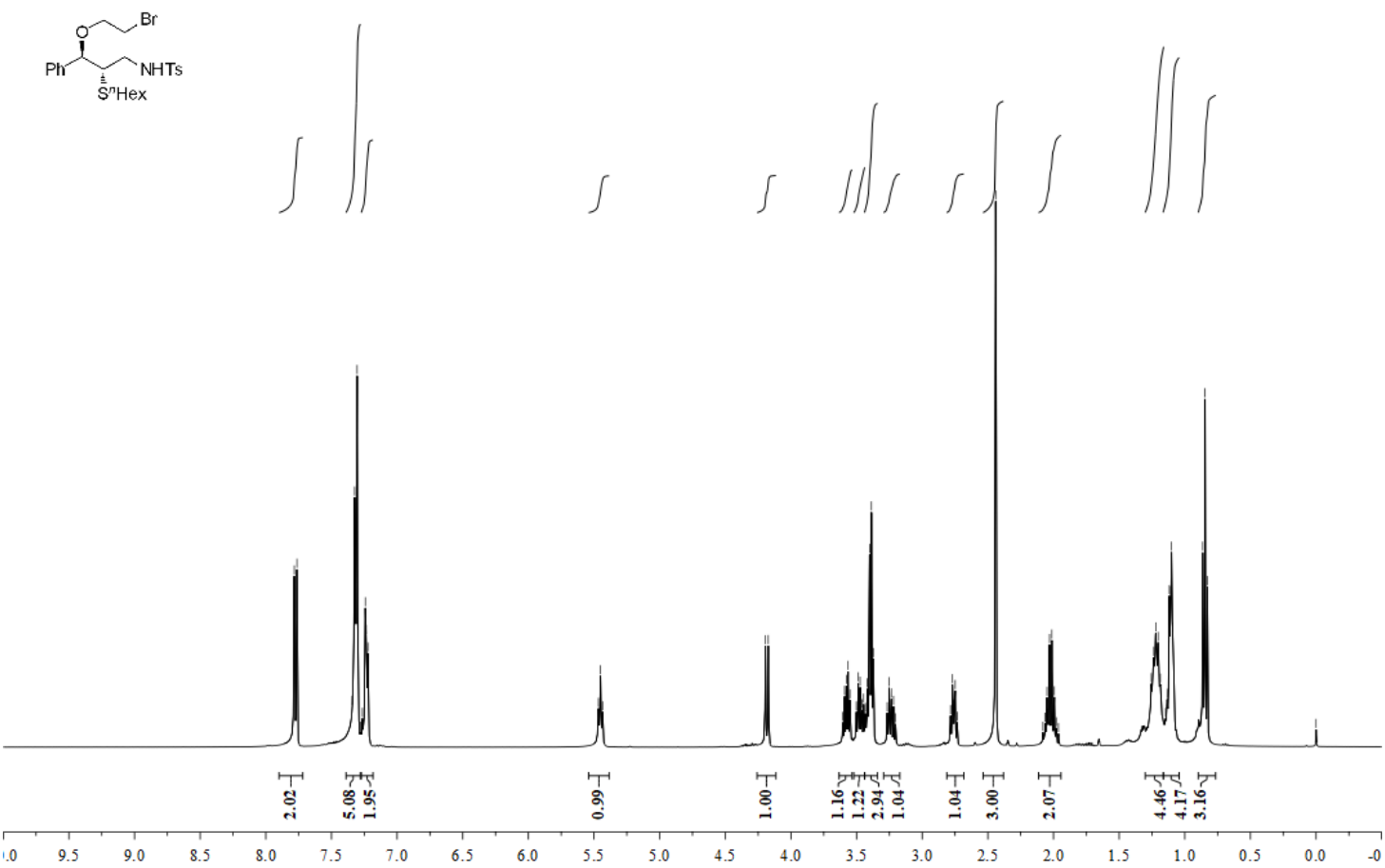

${ }^{13} \mathrm{C}$ NMR (101 MHz, $\left.\mathrm{CDCl}_{3}\right)$ spectrum of compound 4c

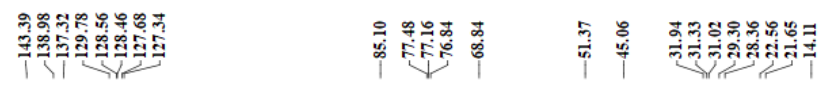

$\overbrace{\mathrm{S}^{n} \mathrm{Hex}}^{\mathrm{NHTS}}$

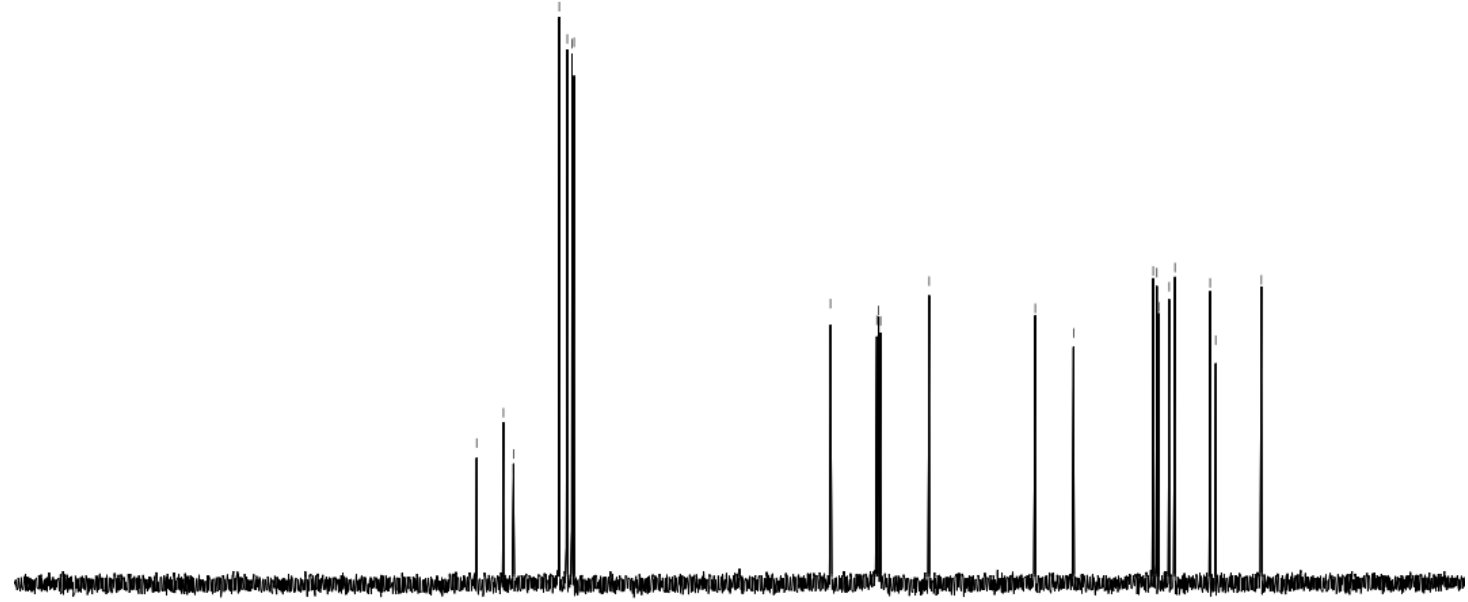

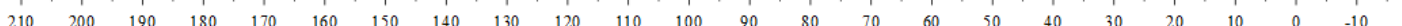


${ }^{1} \mathrm{H}$ NMR (400 MHz, $\left.\mathrm{CDCl}_{3}\right)$ spectrum of compound $\mathbf{4 d}$

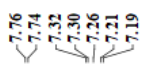

早果的

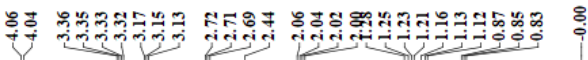
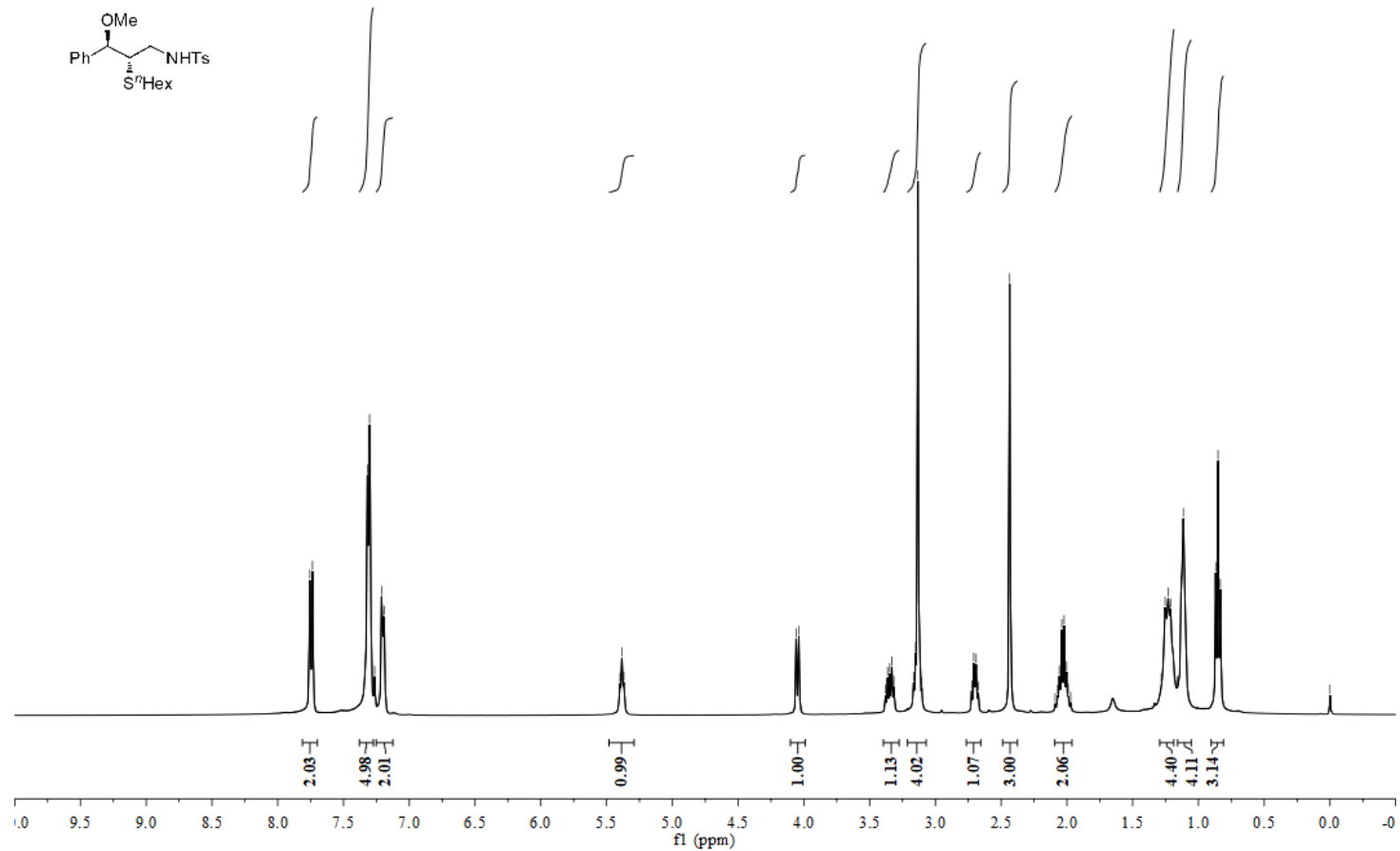

${ }^{13} \mathrm{C}$ NMR (101 MHz, $\left.\mathrm{CDCl}_{3}\right)$ spectrum of compound 4d

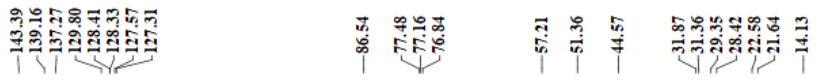

$\overbrace{\substack{S^{n} \mathrm{Hex} \\ \mathrm{NHTS}}}^{\mathrm{MMe}}$

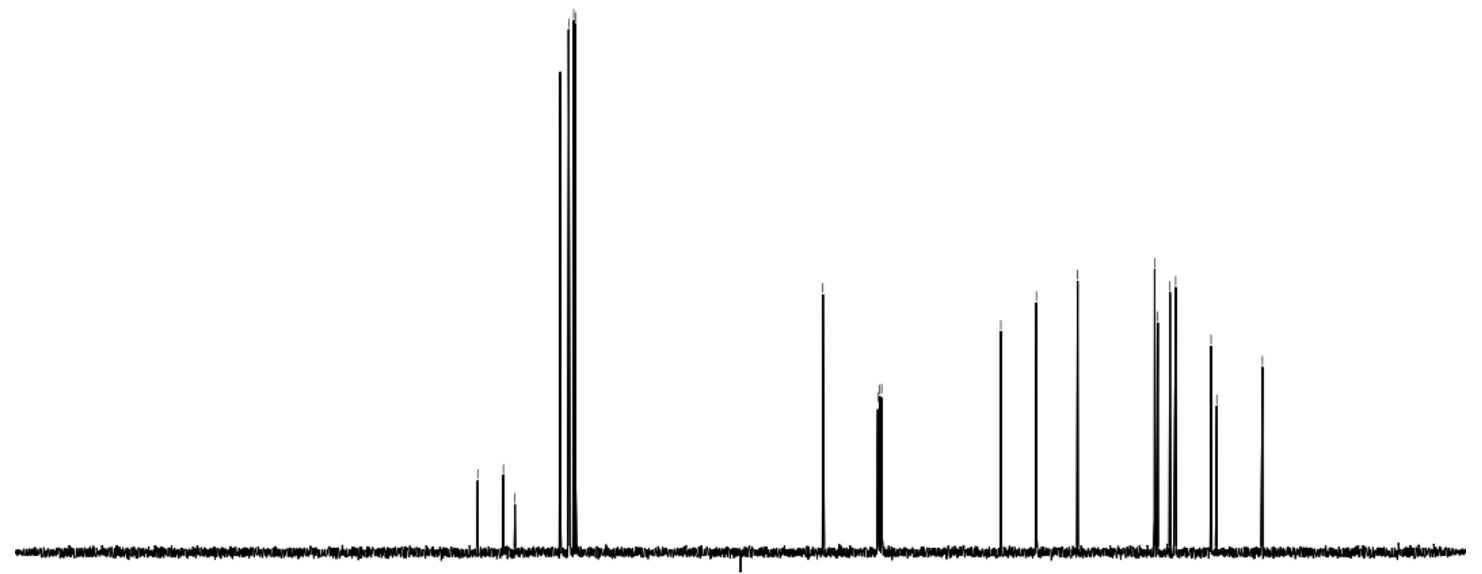

$\begin{array}{lllllllllllllllllllllllllll}210 & 200 & 190 & 180 & 170 & 160 & 150 & 140 & 130 & 120 & 110 & 100 & 90 & 80 & 70 & 60 & 50 & 40 & 30 & 20 & 10 & 0 & -10 & \end{array}$ 
${ }^{1} \mathrm{H}$ NMR (400 MHz, $\mathrm{CDCl}_{3}$ ) spectrum of compound $4 \mathbf{e}$ 施

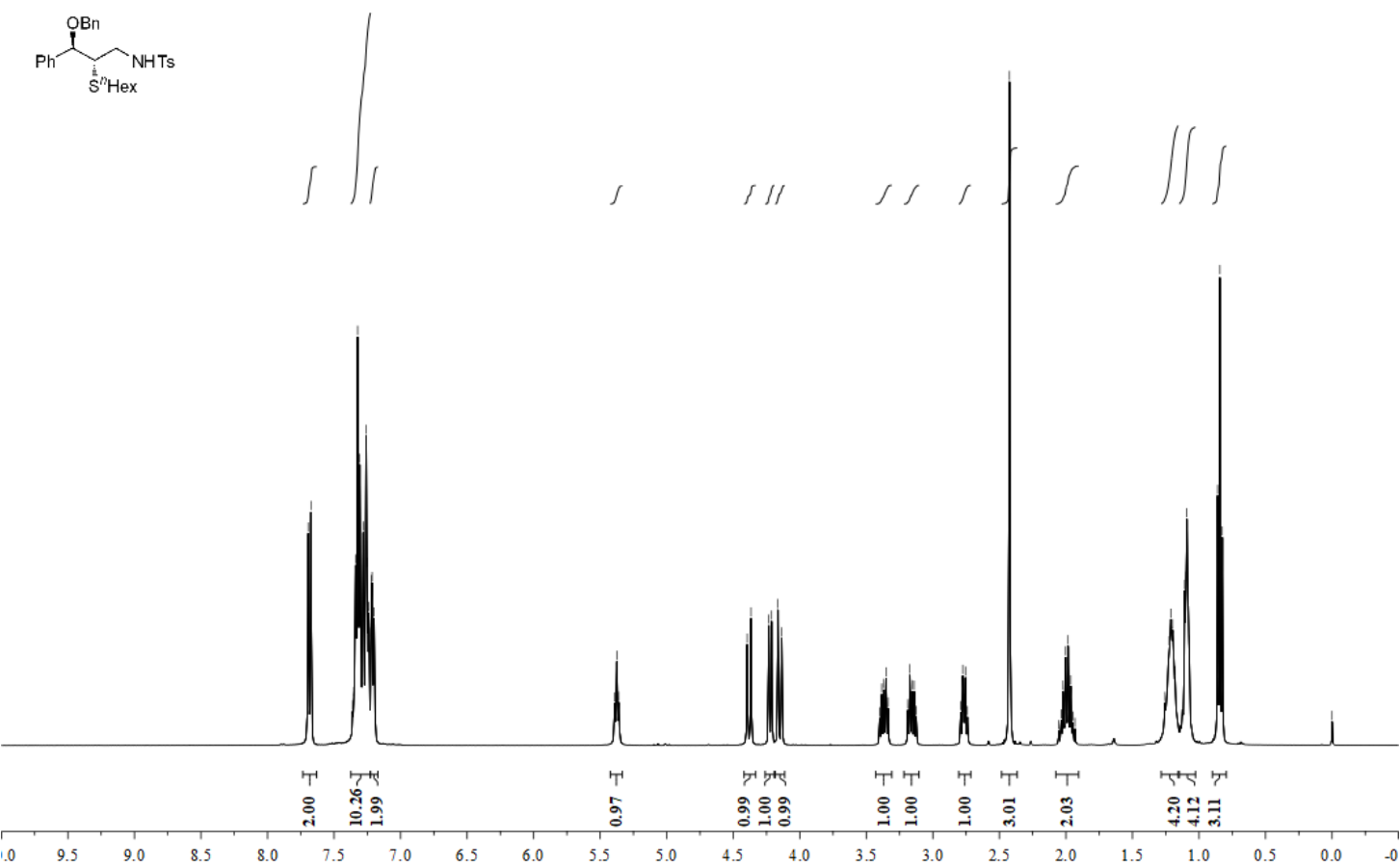

${ }^{13} \mathrm{C}$ NMR (101 MHz, $\left.\mathrm{CDCl}_{3}\right)$ spectrum of compound $\mathbf{4 e}$

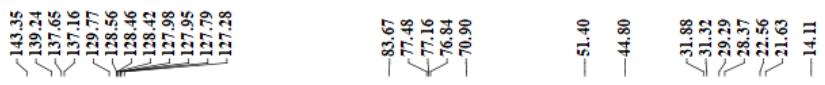

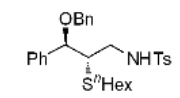


${ }^{1} \mathrm{H}$ NMR (400 MHz, $\mathrm{CDCl}_{3}$ ) spectrum of compound $\mathbf{4 f}$

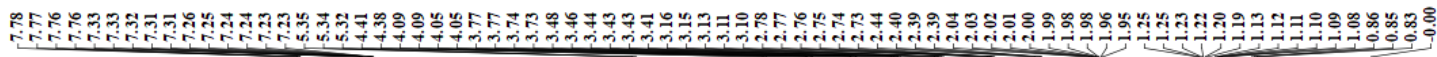

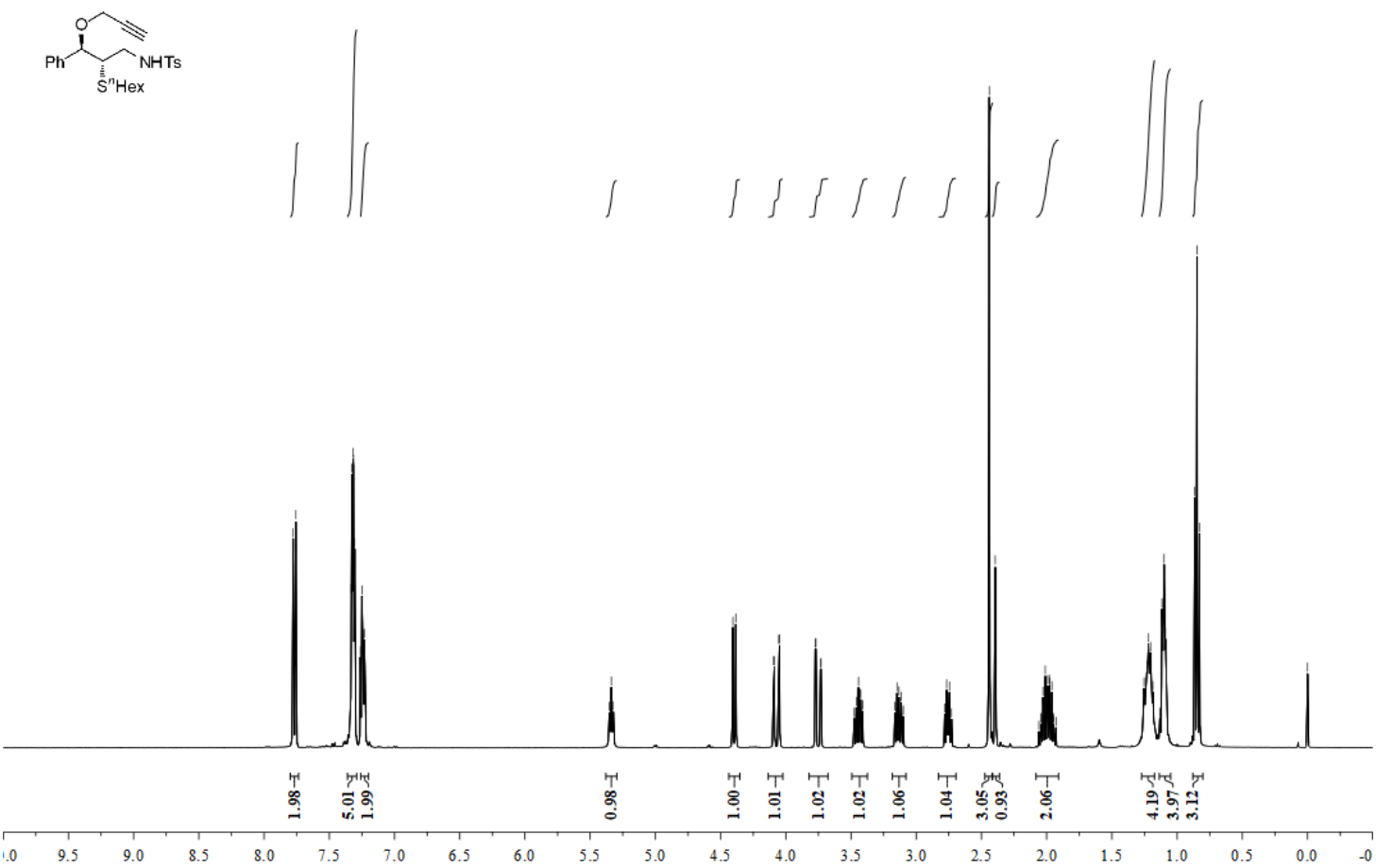

${ }^{13} \mathrm{C}$ NMR $\left(101 \mathrm{MHz}, \mathrm{CDCl}_{3}\right)$ spectrum of compound $\mathbf{4 f}$

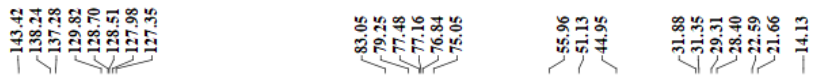

$\overbrace{\mathrm{S}^{\mathrm{Hex}} \mathrm{NHT}}^{\mathrm{NHT}}$

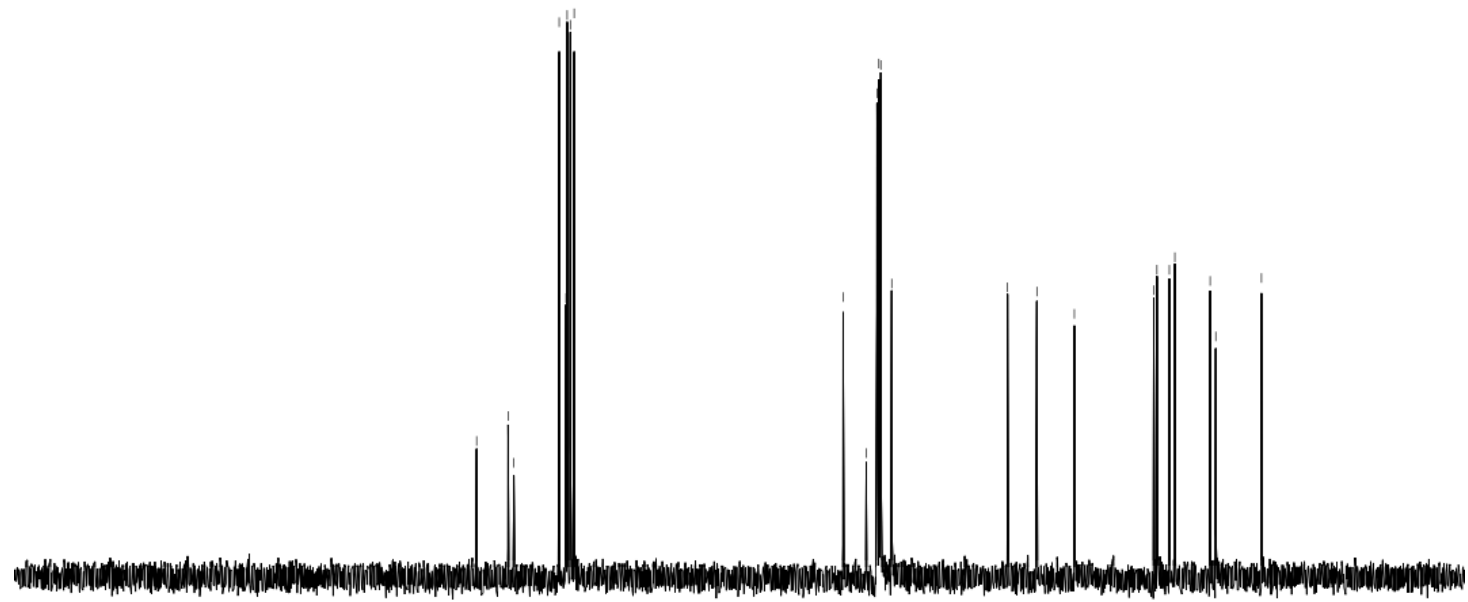

$\begin{array}{lllllllllllllllllllllll}210 & 200 & 190 & 180 & 170 & 160 & 150 & 140 & 130 & 120 & 110 & 100 & 90 & 80 & 70 & 60 & 50 & 40 & 30 & 20 & 10 & 0 & -10\end{array}$ 
${ }^{1} \mathrm{H}$ NMR (400 MHz, $\left.\mathrm{CDCl}_{3}\right)$ spectrum of compound 5

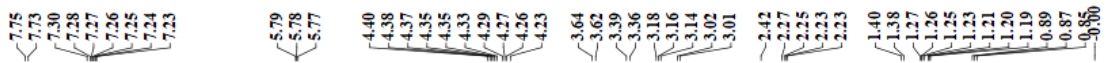

$$
\underbrace{\text { OnTs }}_{\text {STlex }}
$$
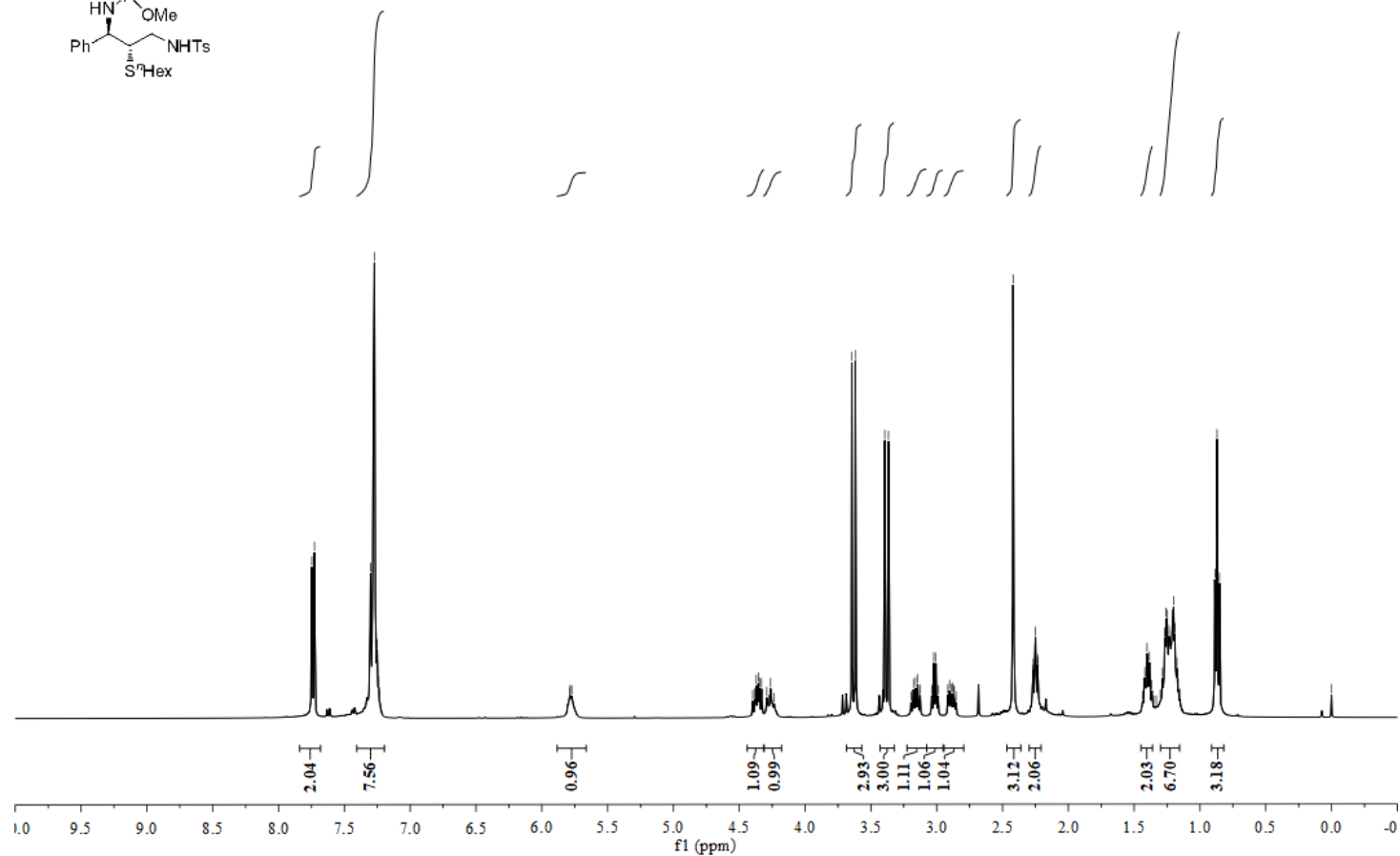

${ }^{13} \mathrm{C}$ NMR (101 MHz, $\left.\mathrm{CDCl}_{3}\right)$ spectrum of compound 5

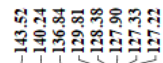

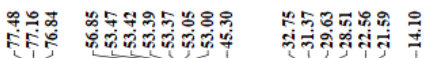

$\underbrace{O}_{S_{S_{H e x}}^{N H T s}}$

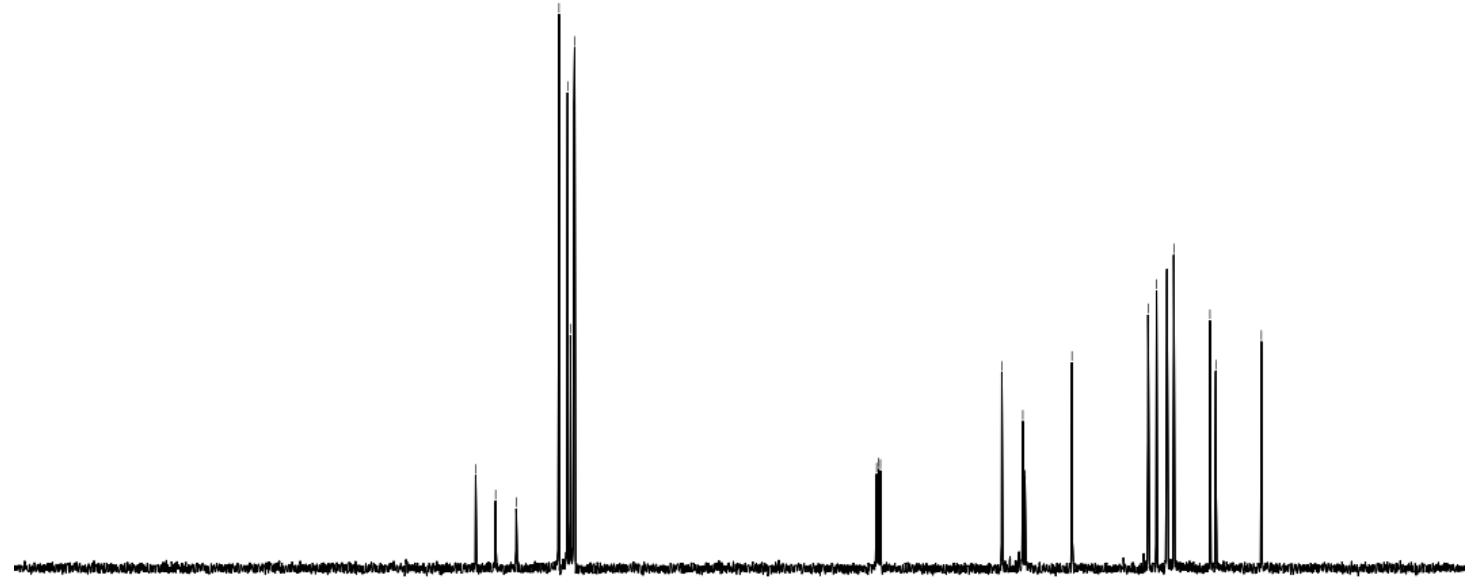

$\begin{array}{llllllllllllllllllllllllllllll}210 & 200 & 190 & 180 & 170 & 160 & 150 & 140 & 130 & 120 & 110 & 100 & 90 & 80 & 70 & 60 & 50 & 40 & 30 & 20 & 10 & 0 & -10 & \end{array}$ 
${ }^{1} \mathrm{H}$ NMR (400 MHz, $\left.\mathrm{CDCl}_{3}\right)$ spectrum of compound 6

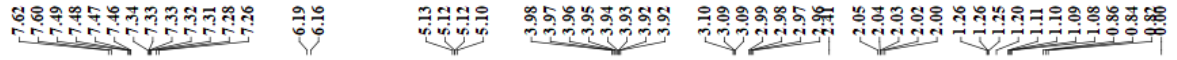
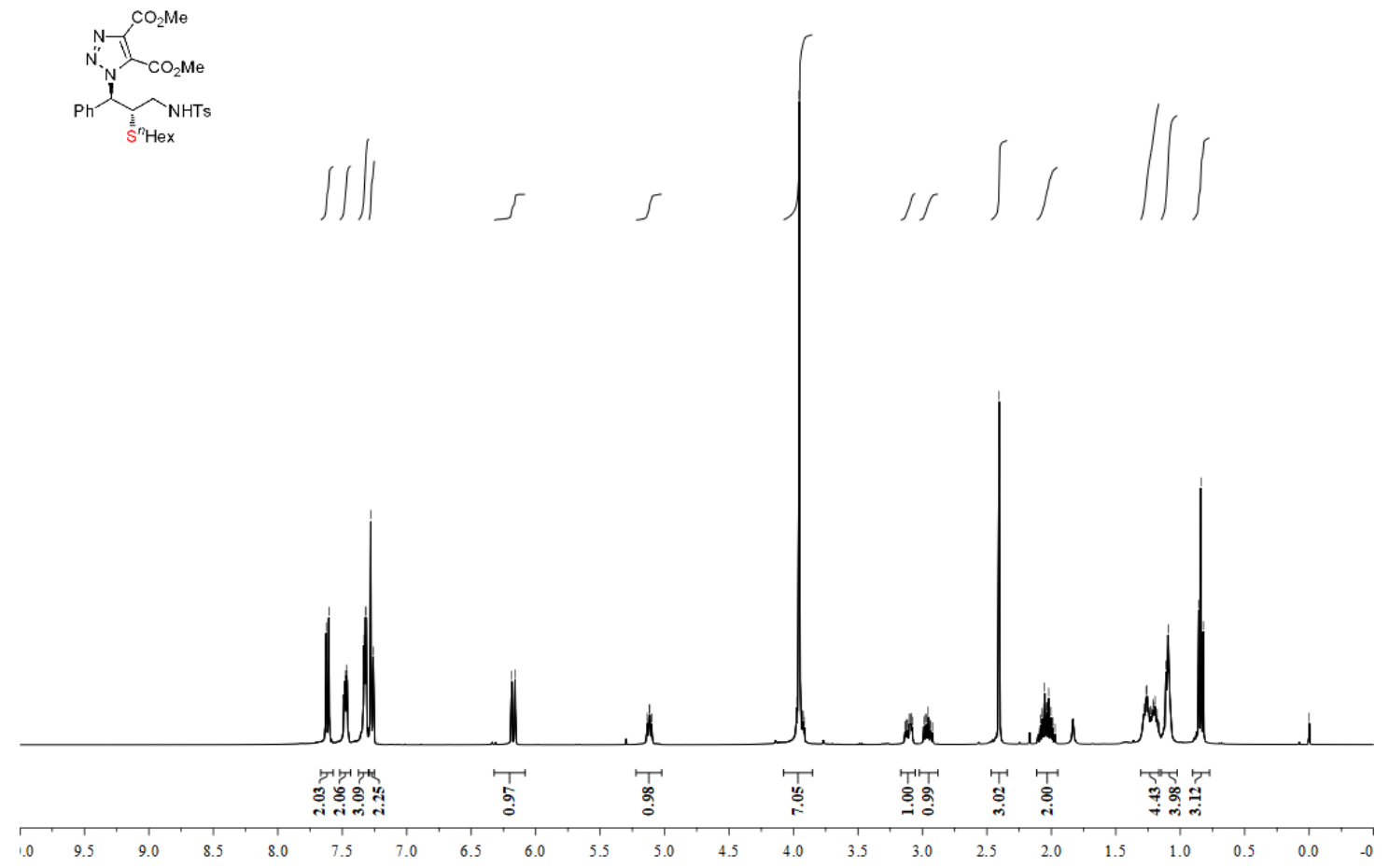

${ }^{13} \mathrm{C}$ NMR (101 MHz, $\left.\mathrm{CDCl}_{3}\right)$ spectrum of compound 6
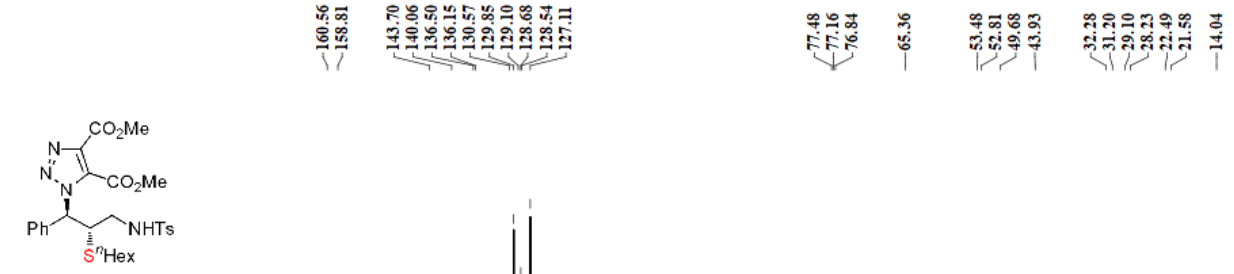

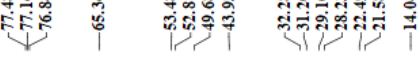

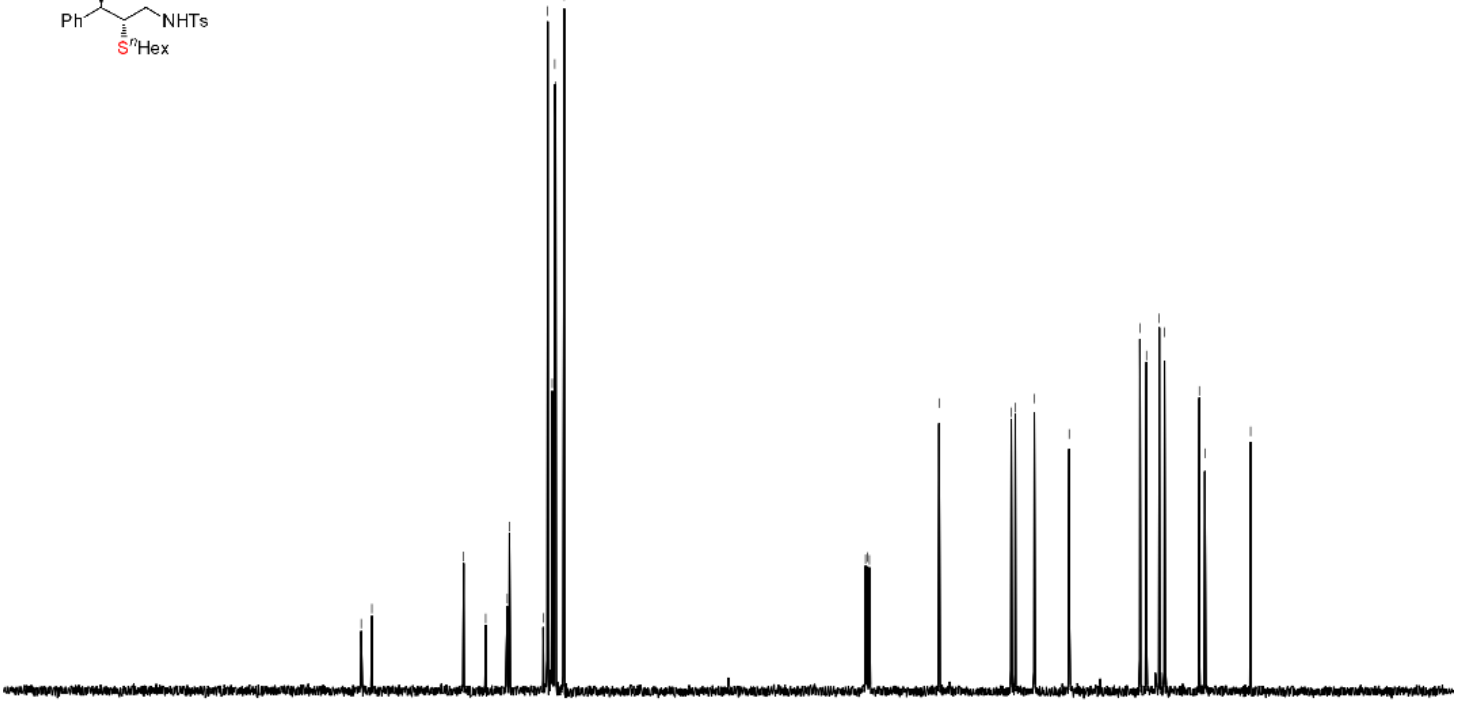

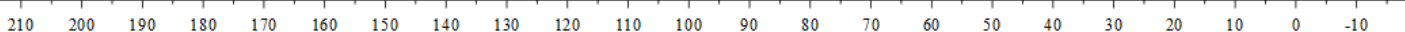


${ }^{1} \mathrm{H}$ NMR (400 MHz, $\mathrm{CDCl}_{3}$ ) spectrum of compound 7

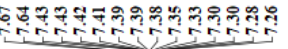

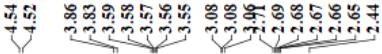
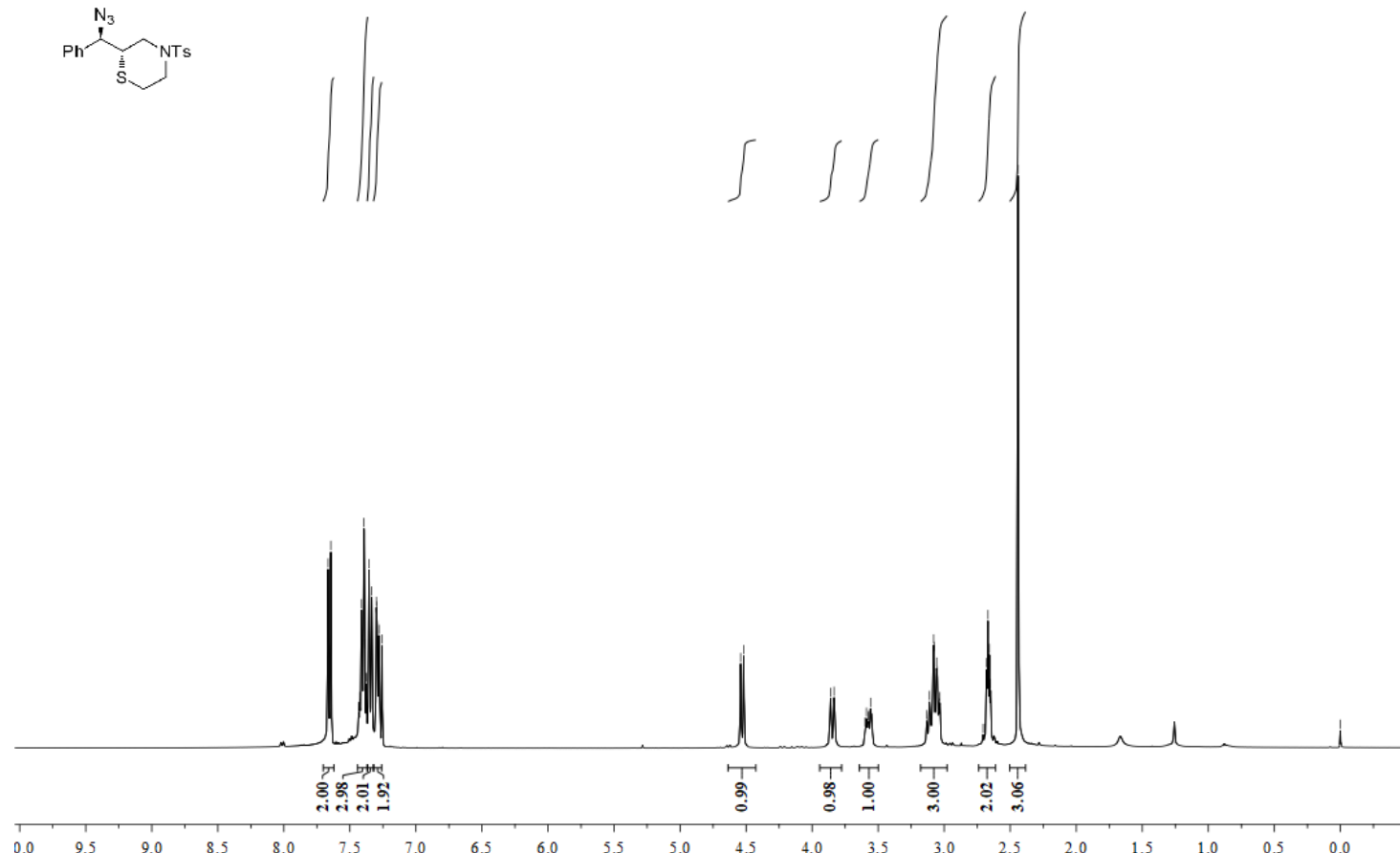

${ }^{13} \mathrm{C}$ NMR (101 MHz, $\left.\mathrm{CDCl}_{3}\right)$ spectrum of compound 7

\begin{tabular}{|c|c|c|}
\hline 40 & 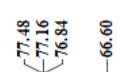 & 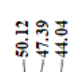 \\
\hline
\end{tabular}<smiles>N[C@H](c1ccccc1)C1[CH][NH2+]CCC1</smiles>

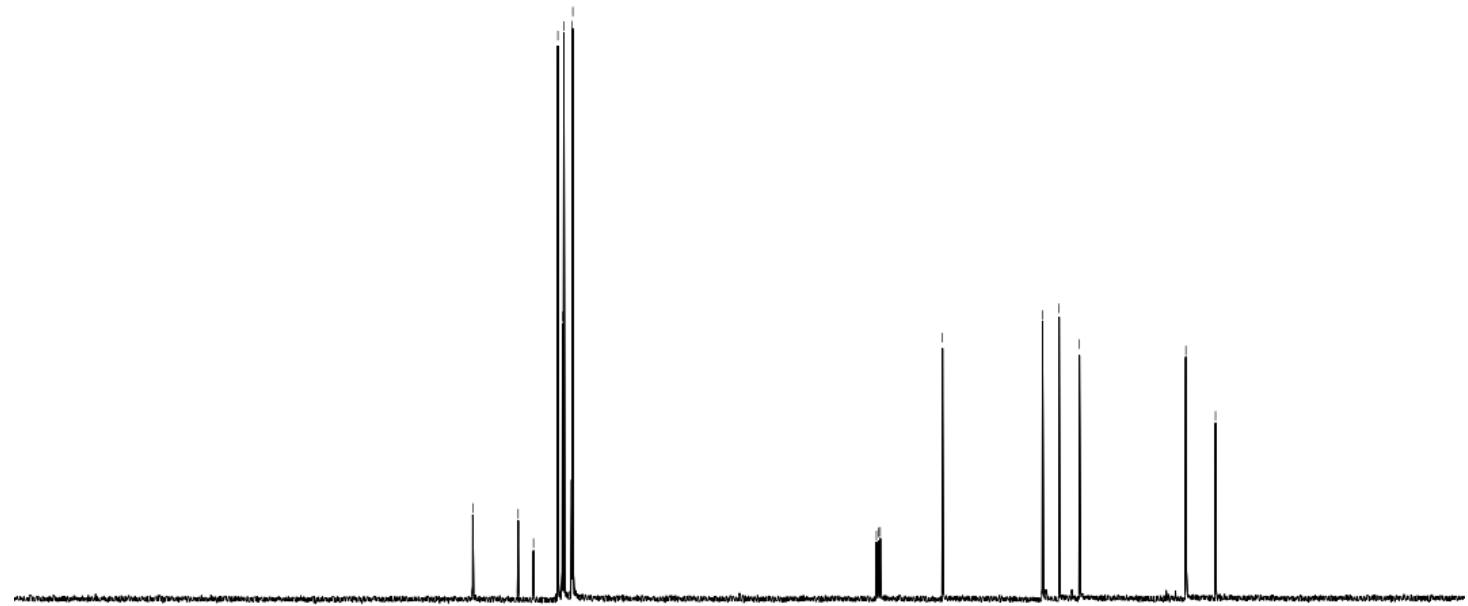

$\begin{array}{llllllllllllllllllllllll}210 & 200 & 190 & 180 & 170 & 160 & 150 & 110 & 130 & 120 & 110 & 100 & 90 & 80 & 70 & 60 & 50 & 40 & 30 & 20 & 10 & 0\end{array}$ 
${ }^{1} \mathrm{H}$ NMR (400 MHz, $\mathrm{CDCl}_{3}$ ) spectrum of compound 8

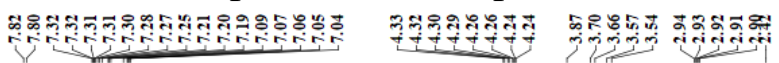
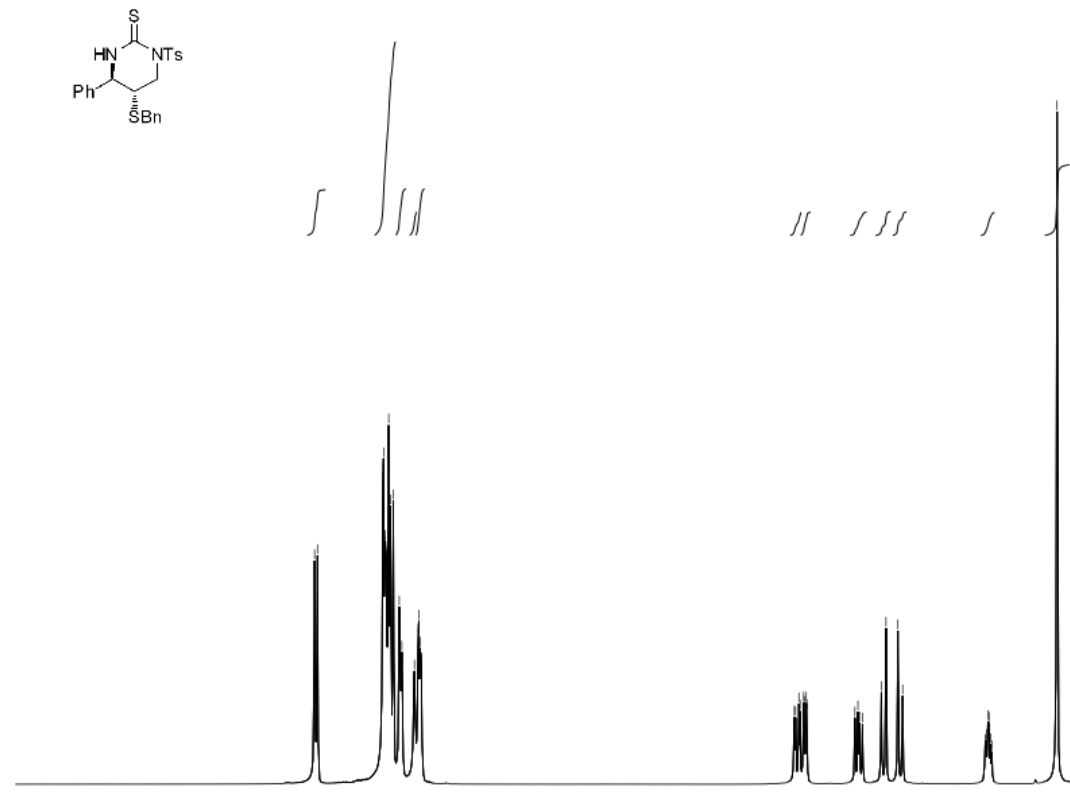

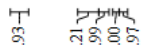

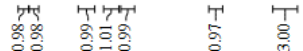

${ }^{13} \mathrm{C}$ NMR (101 MHz, $\mathrm{CDCl}_{3}$ ) spectrum of compound $\mathbf{8}$

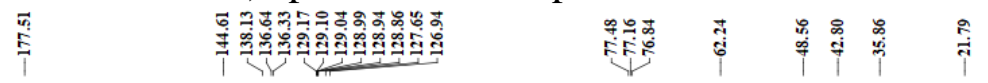

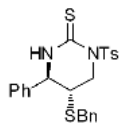

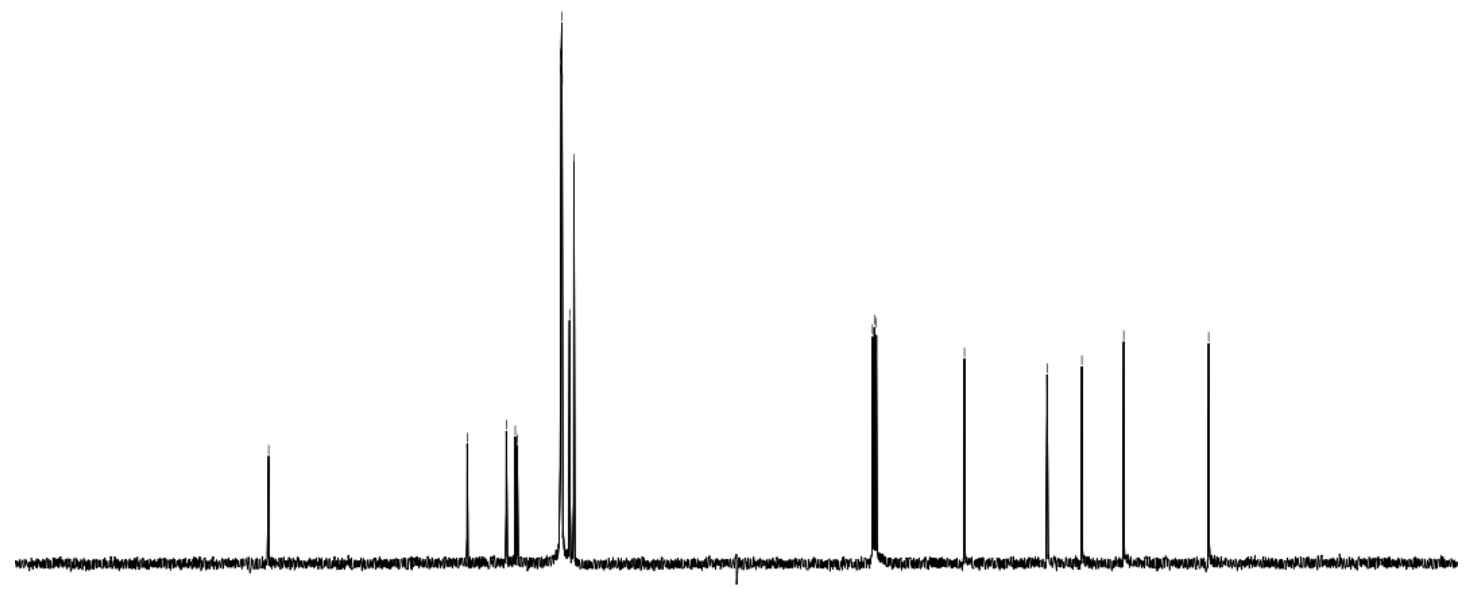

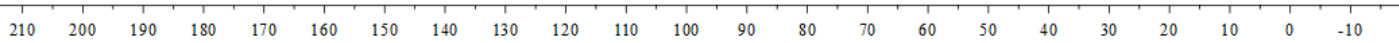


${ }^{1} \mathrm{H}$ NMR (400 MHz, $\mathrm{CDCl}_{3}$ ) spectrum of compound 9

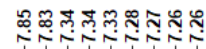

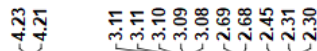

$\stackrel{\circ}{\circ}$

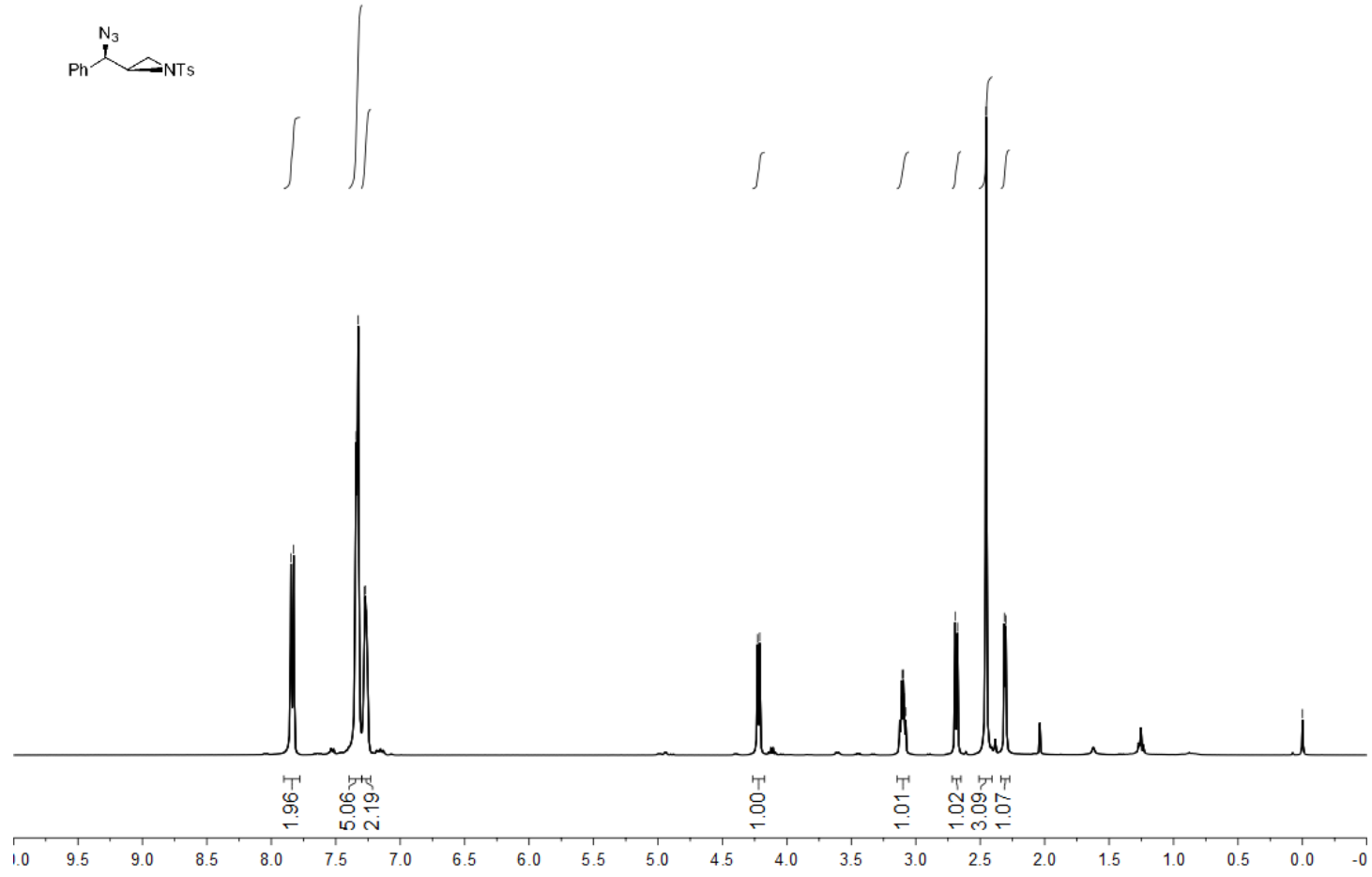

${ }^{13} \mathrm{C}$ NMR (101 MHz, $\mathrm{CDCl}_{3}$ ) spectrum of compound 9

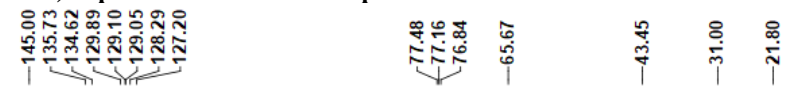

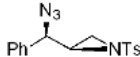

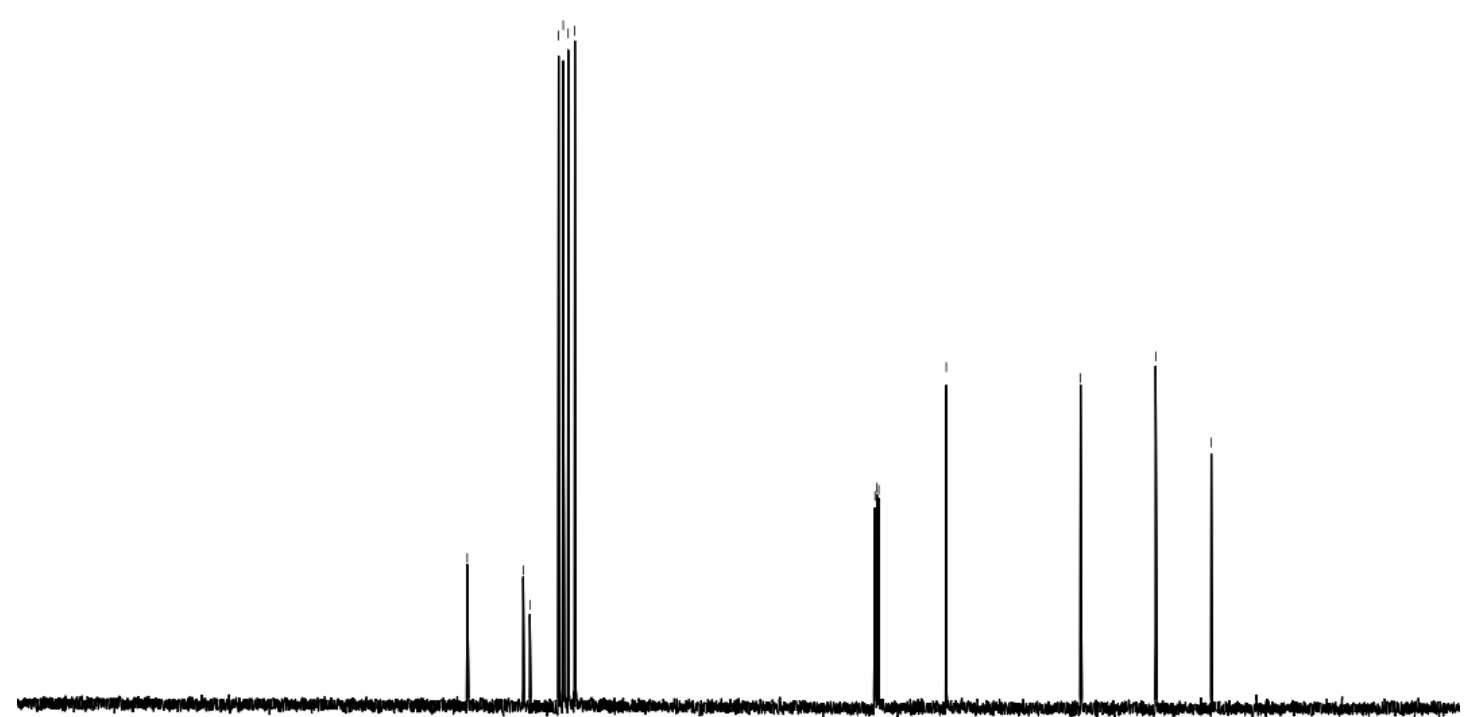

